\title{
WestVirginiaUniversity
}

THE RESEARCH REPOSITORY @ WVU

Graduate Theses, Dissertations, and Problem Reports

2019

\section{Utilization of Scale in Keypoint Detection and Feature Description}

Andrew P. Rhodes

West Virginia University, arhodes5@mix.wvu.edu

Follow this and additional works at: https://researchrepository.wvu.edu/etd

Part of the Navigation, Guidance, Control and Dynamics Commons

\section{Recommended Citation}

Rhodes, Andrew P., "Utilization of Scale in Keypoint Detection and Feature Description" (2019). Graduate Theses, Dissertations, and Problem Reports. 7437.

https://researchrepository.wvu.edu/etd/7437

This Dissertation is protected by copyright and/or related rights. It has been brought to you by the The Research Repository @ WVU with permission from the rights-holder(s). You are free to use this Dissertation in any way that is permitted by the copyright and related rights legislation that applies to your use. For other uses you must obtain permission from the rights-holder(s) directly, unless additional rights are indicated by a Creative Commons license in the record and/ or on the work itself. This Dissertation has been accepted for inclusion in WVU Graduate Theses, Dissertations, and Problem Reports collection by an authorized administrator of The Research Repository @ WVU.

For more information, please contact researchrepository@mail.wvu.edu. 


\title{
Utilization of Scale in Keypoint Detection and Feature Description
}

\author{
Andrew Patrick Rhodes, B.S., B.S. \\ DISSERTATION submitted to the \\ Benjamin M. Statler College of Engineering and Mineral Resources \\ at West Virginia University \\ in partial fulfillment of the requirements for the degree of \\ DOCTOR OF PHILOSOPHY in Aerospace Engineering \\ John A. Christian, III, Ph.D., Chair \\ Jason Gross, Ph.D. \\ $\mathrm{Yu} \mathrm{Gu}, \mathrm{Ph} . \mathrm{D}$. \\ Guilherme Pereira, Ph.D. \\ Stacie Williams, Ph.D.
}

Department of Mechanical and Aerospace Engineering

Morgantown, West Virginia

2019

Keywords: scale-space, diffusion, keypoints, feature histogram descriptor, Laplace-Beltrami

Copyright 2019 Andrew Patrick Rhodes 


\title{
ABSTRACT \\ Utilization of Scale in Keypoint Detection and Feature Description
}

\author{
Andrew Patrick Rhodes, Ph.D.
}

Ubiquitous availability of inexpensive three dimensional (3D) sensors has led to an abundance of keypoint detection and feature description techniques for point-clouds. While some recent methods utilize color along with geometric information, most only implicitly discuss - if not completely disre-

gard - the information provided by scale. Scale is an inherent characteristic of any keypoint or feature that describes its physical size or region of support.

Exploiting information provided by scale facilitates the processes of keypoint detection and feature description. A 3D scale-space, extended from robust and popular 2D methods, is constructed to diffuse a signal on triangulated meshes. Methods of scale-parameter estimation and a novel neighborhood definition are presented that improve the methods' robustness to noise and arbitrary mesh connectivity. Keypoint repeatability experiments show that a novel definition of the Laplace-Beltrami operator (LBO) leads to the most accurate scale-space. 
A point-cloud feature descriptor, popular in the robotics community and recently proposed for spacecraft relative navigation (RelNav), is modified to explicitly utilize scale in the description and matching processes. The Scaled Oriented Unique Clustered Viewpoint Feature Histogram (SOUR-CVFH ) uses a cluster's scale to expedite the histogram matching procedure and increase pose estimation accuracy. SOUR-CVFH is demonstrated in experiments of (1) object recognition in both isolated and cluttered scenes and (2) simulated RelNav of a spacecraft to a asteroid. 


\section{Table of Contents}

Abstract $\quad$ ii

List of Figures $\quad$ x

$\begin{array}{ll}\text { List of Tables } & \text { xviii }\end{array}$

$\begin{array}{ll}\text { List of Publications } & \text { xx }\end{array}$

$\begin{array}{ll}\text { Acknowledgments } & \text { xxi }\end{array}$

Chapter 1. Introduction $\quad 1$

1.1 Motivation .......................... 3

1.1.1 Sensor Development and Support . . . . . . . . . . 3

1.1.2 Uncorrelated Pose Estimates in Navigation Filters . . . 5

1.1.3 Scale-Space Feature Detection in 2D . . . . . . . 7

1.2 Contributions ................... . . 8

1.2.1 Signal Diffusion on 3D Surfaces . . . . . . . . . 8

1.2.2 Scale-space Construction and Keypoint Detection . . . . 9

1.2.3 Scale in Clustered Viewpoint Feature Histograms . . . . 9

1.2.4 Performance Assessments . . . . . . . . . . . . . . . . . 10

Chapter 2. Review of Point-Cloud Feature Detection 11

2.1 Keypoint Detection in Scale-Space . . . . . . . . . . . . . . 11

2.1.1 Physical Scale Keypoints (PSK) . . . . . . . . . . . . . 12

2.1.2 Geodesic Scale-Space (GSS) . . . . . . . . . . . . 15

2.1.3 Hierarchical Scale-Space (HSS) . . . . . . . . . . . . . . 17

2.1.4 Mesh SIFT . . . . . . . . . . . . . . . 18

2.1.5 ThrIFT . . . . . . . . . . . . . . . . 19

2.1.6 Mesh Difference of Gaussian (MeshDoG) . . . . . . . . 21 
2.1.7 Curvature Scale-Space 3D (CS3) _ . . . . . . . . . 23

2.2 Feature Histogram Descriptors . . . . . . . . . . . . . . . 24

2.2.1 Persistent Point Feature Histogram (PFH) . . . . . . . 25

2.2.2 Fast Point Feature Histogram (FPFH) . . . . . . . 30

2.2 .3 Viewpoint Feature Histogram (VFH) . . . . . . . . . 33

2.2.4 Clustered Viewpoint Feature Histogram (CVFH) . . . . 37

2.2.5 Oriented Unique Repeatable Clustered Viewpoint Feature Histogram (OUR-CVFH) _ . . . . . . . . . . . 43

2.2.5.1 Viewpoint Clustering . . . . . . . . . . . . 44

2.2.5.2 Semi-Global Unique Reference Frame (SGURF) 46

2.2.5.3 Histogram Generation . . . . . . . . . . . 51

2.2.5.4 Database Training . . . . . . . . . . . . 56

2.2.5.5 Recognition Framework _. . . . . . . 58

Chapter 3. Signal Diffusion on 3D Meshes and Point-Clouds 62

3.1 Surface, Mesh, and Grid Definitions . . . . . . . . . 63

3.1 .1 Surfaces and Meshes . . . . . . . . . . . . . 64

3.1 .2 Embedding Grids . . . . . . . . . . . . . . 65

3.2 Discretized Diffusion on Implicit Surfaces (DI) . . . . . . . 66

3.2.1 Diffusion with the Implicit Closest Point Method (ICPM) 68

3.2 .2 Mesh Diffusion Examples . . . . . . . . . . . . 70

3.2.2.1 Diffusion of an Impulse . . . . . . . . . . . 71

3.2.2.2 Diffusion of a Monochrome Image . . . . . . . 73

3.2 .3 Comments . . . . . . . . . . . . . . . . 74

3.3 Gaussian Diffusion on Implicit Surfaces (GI) . . . . . . . . 75

3.3.1 Diffusion with the Closest Point Method (CPM) . . . 75

3.3.2 Determining the Bandwidth . . . . . . . . . 76

3.3.3 Determining the Kernel Window and Width . . . . . . 77

3.3 .4 Comments . . . . . . . . . . . . . . . . 79

3.4 Discretized Diffusion on Explicit Surfaces (DE) . . . . . . 79

3.4.1 Diffusion with discrete Laplacians _ . . . . . . . . 79

3.4 .2 Laplace-Beltrami Operators (LBO) . . . . . . . . . . . . . 81

3.4.2.1 Umbrella Laplace Operator . . . . . . . . . . . 82 
3.4.2.2 Cotangent Laplace Operator . . . . . . . . . . . 84

3.4.2.3 Mesh Laplace Operator . . . . . . . . . . . . . . 85

3.4.2.4 Effects of Mesh Scaling . . . . . . . . . . . . 87

3.4.3 Mesh Diffusion Examples . . . . . . . . . . . . . . . 88

3.4.3.1 Diffusion of an Impulse . . . . . . . . . . . . 89

3.4.3.2 Diffusion of a Monochrome Image . . . . . . . . 90

3.4.3.3 Diffusion of a 3D Model . . . . . . . . . . . . . 91

3.5 Gaussian Diffusion on Explicit Surfaces (GE) . . . . . . . . 92

3.5.1 Diffusion with discrete Gaussians . . . . . . . . . . . 98

3.5.2 Mesh Diffusion Examples . . . . . . . . . . . . . . 99

3.5.2.1 Diffusion of an Impulse . . . . . . . . . . . . 101

3.5.2.2 Diffusion of a Monochrome Image . . . . . . . . 102

3.5.2.3 Diffusion of a 3D Model . . . . . . . . . . . 102

3.6 Conclusions and Comments . . . . . . . . . . . . . . . 108

$\begin{array}{lll}\text { Chapter 4. Scale-Space Construction } & 109\end{array}$

4.1 Scale-Parameter Selection and Estimation . . . . . . . . . . 110

4.1 .1 Time Step Selection . . . . . . . . . . . . . . . . 111

4.1.1.1 Selecting the Time Step for Method DE . . . . 112

4.1.1.2 Selecting the Time Step for Method GE . . . . 113

4.1.1.3 Selecting the Number of Levels . . . . . . . . . 114

4.1 .2 Estimation Methods . . . . . . . . . . . . . . . . . 115

4.1.2.1 Quadratic Fitting Method . . . . . . . . . . 116

4.1.2.2 Cutoff Method . . . . . . . . . . . . . . 117

4.1.2.3 Gaussian Relationship Method . . . . . . . . 118

4.1.2.4 Numerical Example . . . . . . . . . . . . . . . 118

4.2 Keypoint Detection, Localization, and Automatic Scale Selection 119

4.2.1 Redefining the Neighborhood . . . . . . . . . . . . . 122

4.2 .2 Non-Maximum Suppression (NMS) . . . . . . . . . . . 123

4.2.2.1 NMS for Method DE . . . . . . . . . . . . . 124

4.2.2.2 NMS for Method GE . . . . . . . . . . . . 125

4.3 Keypoint Detection Examples . . . . . . . . . . . . . 125 
4.3.1 Planar Mesh Example using DE . . . . . . . . . . . 126

4.3.2 Planar Mesh Example using GE . . . . . . . . . . . . 128

4.3.3 General Mesh Example using DE . . . . . . . . . . . . 129

4.3.4 General Mesh Example using GE . . . . . . . . . . . . 129

4.4 Numerical Validation of Keypoint Detection . . . . . . . . . 131

4.4 .1 Repeatability Metrics . . . . . . . . . . . . . . . 134

4.4.2 Keypoint Repeatability Results . . . . . . . . . . . 136

4.4.2.1 Repeatability of DE adding Signal Noise . . . . 137

4.4.2.2 Repeatability of DE adding Vertex Noise . . . . 140

4.4.2.3 Repeatability of GE adding Signal Noise . . . . 142

4.4.2.4 Repeatability of GE adding Vertex Noise . . . . 144

4.5 Comments on Scale-Space Diffusion Methods . . . . . . . . . 146

Chapter 5. Feature Histogram Descriptors 148

5.1 Application of OUR-CVFH to LIDAR-Based Relative Navigation149

5.1 .1 Training . . . . . . . . . . . . . . . . . . . . . . . . . 149

5.1 .2 Recognition Framework . . . . . . . . . . . . . 150

5.1.3 Uniqueness of Feature Histograms and Pose Observability 150

5.1.4 Numerical Example: Rendezvous with an Asteroid . . . 154

5.1.4.1 Problem Scenario and Monte Carlo Set-up . . . 154

5.1.4.2 Unfiltered OUR-CVFH Pose Performance . . . 155

5.1.4.3 Processing with a Simple Multiplicative Extended Kalman Filter (MEKF) . . . . . . . . . . 155

5.1.4.4 Brief Review of MEKF Implementation . . . . . 157

5.1.4.5 Filtered OUR-CVFH Pose Performance . . . . . 159

5.1.5 Comments on the use of OUR-CVFH in Space Applications160

5.2 Development of Scaled OUR-CVFH (SOUR-CVFH) . . . . . 164

5.2 .1 Linear Interpolation . . . . . . . . . . . . . 170

5.2.2 Histogram Component Description Space . . . . . . . 171

5.2 .3 Pose Estimation Cost Metric . . . . . . . . . . . . 176

5.2 .4 Histogram Normalization . . . . . . . . . . . . . . 180

5.2 .5 Distance Metric Selection . . . . . . . . . . . . . 181

5.2 .6 Cluster Scale as an Identifier $\ldots \ldots \ldots$ 
5.2.7 Interpolated Spatial Octant Component (ISOC) . . . . . 200

5.2.8 Uniqueness of Histogram Components . . . . . . . . . . 212

5.2 .9 Reference Frame Construction . . . . . . . . . . . . . . 218

5.2.10 Comments . . . . . . . . . . . . . . 227

5.3 Scaled OUR-CVFH (SOUR-CVFH) . . . . . . . . . . . . . . 228

5.3.1 Descriptor Construction . . . . . . . . . . . . . . 229

5.3.1.1 Viewpoint Clustering . . . . . . . . . . . 229

5.3.1.2 Semi-Global Unique Reference Frame (SGURF) and Object Scale . . . . . . . . . . . . 231

5.3.1.3 Histogram Construction . . . . . . . . . . 236

5.3.2 Training Database Construction . . . . . . . . . . 240

5.3.3 Recognition Framework . . . . . . . . . . . . . . 241

5.3.4 Comments on SOUR-CVFH . . . . . . . . . . 243

5.4 Scaled and Oriented Locally CVFH (SOL-CVFH) . . . . . . . 246

5.4.1 Local Reference Frame (LRF) and Object Scale . . . . . 246

5.4 .2 Comments on SOL-CVFH . . . . . . . . . . . . 248

Chapter 6. Feature Histogram Performance Assessment 250

6.1 Individual Object Pose Estimation . . . . . . . . . . . . . . . . 250

6.2 Object Recognition in Cluttered Scenes . . . . . . . . . . . . . 261

6.3 Rendezvous with an Asteroid . . . . . . . . . . . . . . . . 274

6.3.1 Problem Scenario . . . . . . . . . . . . . . 274

6.3.2 Unfiltered Pose Performance . . . . . . . . . . . . 275

6.3.3 Filtered Pose Performance . . . . . . . . . . . . . 279

Chapter 7. Conclusions $\quad \mathbf{2 8 6}$

7.1 Conclusions . . . . . . . . . . . . . . . . . 286

7.2 Future Work . . . . . . . . . . . . . . . . . . . . . . 289

7.2.1 Multi-Scale Framework for 3D Meshes . . . . . . . . . . 289

7.2.2 Incorporate Scale into Random Keypoint Feature Descriptors . . . . . . . . . . . . 290

7.2.3 Geometric Consistency of Clustered Features . . . . . . 291

7.2.4 Inclusion of Scene Color Cues with SOUR-CVFH and SOL-CVFH . . . . . . . . . . . . . . . 292

7.2.5 Multi-Feature Combinations . . . . . . . . . . 293 
Bibliography

Vita

319 


\section{List of Figures}

\section{Chapter 1. Introduction}

1.1 Example of information reuse by a Kalman Filter (KF) for a sensor that estimates pose. . . . . . . . . . .

\section{Chapter 2. Review of Point-Cloud Feature Detection}

2.1 A cluster in blue with the rest of the measured point-cloud in red. The SGURF is centered on the cluster's centroid. To aid in disambiguation, the $x$-axis of the SGURF directs towards the rest of the point-cloud and along the body of Itokawa. . . . . .

2.2 A Darboux frame $\mathrm{D}$ is a local right handed coordinate system for a point on a patch. Notice that $\mathbf{u}$ is the parallel to the average normal $\mathbf{n}_{c}$. When looking at the patch from the camera viewpoint, $\mathbf{v}$ directs counter-clockwise for all points. Also notice that $\mathbf{w}$ is nearly parallel with the line connecting the point and the cluster centroid, and is directed towards the centroid $\overline{\mathbf{p}}_{c}$.

2.3 To better understand the meaning of the feature angles, consider the local Darboux Frame at an arbitrary point $\mathbf{p}_{i}$ defined as $\mathrm{D}=(\mathbf{u}, \mathbf{v}, \mathbf{w})$. The angle between $\mathbf{v}$ and the surface normal $\mathbf{n}_{i}$ at $\mathbf{p}_{i}$ is $\alpha$. The angle between $\mathbf{u}$ and the line connecting the cluster centroid $\overline{\mathbf{p}}_{c}$ to $\mathbf{p}_{i}$ is $\phi$. The angle between $\mathbf{n}_{i}$ and the line parallel to the line connecting the camera to $\overline{\mathbf{p}}_{c}$ is $\beta$. The meaning of $\theta$ is difficult to explain graphically so is not shown.

2.4 An example of the OUR-CVFH histogram. The features are concatenated and color labeled. The size of the patch is implicitly contained in the histogram as it counts the total number of points in the patch. . . . . . . . . . . . . . 
2.5 Illustration of the best match between a set of feature histograms found in a measured 3D point-cloud (red box) and a catalog of known feature histograms (blue box). Every observed scene feature histogram is matched to every catalog feature histogram. The best match (as determined by Sec. 2.2.5.5) is the pair of overlaid histograms in the center. For histograms from the observed scene, their corresponding cluster is shown in the image of Itokawa. Note here that the largest cluster (the magenta cluster) was not used to estimate pose. This process is influenced by the parameter $\lambda$ in Eq. 2.91. . . . . . . . . . . . 59

2.6 Example OUR-CVFH clusters . . . . . . . . . . . . 61

\section{Chapter 3. Signal Diffusion on 3D Meshes and Point-Clouds}

3.1 Triangular mesh definitions. A vertex $\mathbf{v}_{i}$ is connected by an edge $e_{i j}$ on the face $f_{i}$. . . . . . . . . . . . . . .

3.2 Embedding grid definitions. A mesh $\mathcal{M}$ is a circle, embedded in a grid of $\mathcal{B}$ (indicated by $\bullet$ ), and the ghost points $\mathcal{G}$ (indicated by $\circ$ ). For a point on $\mathcal{M}$ (indicated with $\diamond$ ), the $4 \times 4$ interpolation stencil is shown in purple. The five point Laplace stencil is shown in red. This figure is reproduced from [1]. . . . . . . . 65

3.3 A general mesh compared to the simplicity of a planer mesh. . 71

3.4 Gaussian diffusion of an impulse on a 2D image . . . . . . . . 72

3.5 Impulse diffusion on an implicit planar mesh using DI . . . . . 73

3.6 Implicit surface diffusion of Comet67P/CG . . . . . . . . . . . 74

3.7 Visualization of the LBO sparsity . . . . . . . . . . . . . . 83

3.8 Graphical representation of the mixed area cotangent LBO. . . 86

3.9 Diffusion of impulse on planar mesh using DE . . . . . . . . . 89

3.10 DE diffusion for decreasing edge length . . . . . . . . . . . . . 90

3.11 Planar surface diffusion of Comet67P/CG with method DE . . 91

3.12 Armadillo model diffusion using DE . . . . . . . . . . . . . . 93

3.13 Buddha model diffusion using DE . . . . . . . . . . . . . . . . 94

3.14 Bunny model diffusion using DE . . . . . . . . . . . . . . . . . . . . . . . . . . . . . . 95

3.15 Dragon model diffusion using DE . . . . . . . . . . . . . 96

3.16 Itokawa model diffusion using DE . . . . . . . . . . . . . . . 97

3.17 Visualization of the explicit Gaussian sparsity . . . . . . . . . 100

3.18 Diffusion of impulse on planar mesh using GE . . . . . . . . . 101 
3.19 Planar surface diffusion of Comet67P/CG with method GE . . 103

3.20 Armadillo model diffusion using GE . . . . . . . . . . . . . . . 104

3.21 Buddha model diffusion using GE . . . . . . . . . . . . . . . . 105

3.22 Bunny model diffusion using GE . . . . . . . . . . . . . . . 106

3.23 Dragon model diffusion using GE . . . . . . . . . . . . . . . . 107

3.24 Itokawa model diffusion using GE . . . . . . . . . . . . . . . . 108

\section{Chapter 4. Scale-Space Construction}

4.1 Transfer Function Approximation of Gaussian . . . . . . . . . 119

4.2 First-ring neighbor comparison for three consecutive scale-space levels . . . . . . . . . . . . . . . . . . . . 121

4.3 Compare first-ring and distance-ring neighborhoods . . . . . . 121

4.4 Compare DE to SIFT keypoints on planar mesh . . . . . . . . 127

4.5 Compare GE to SIFT keypoints on planar mesh . . . . . . . . 128

4.6 Models from the Standford scanning repository and the asteroid Itokawa with keypoints using each of the LBOs. . . . . . . . . 130

4.7 Models from the Standford scanning repository and the asteroid Itokawa with keypoints using method GE with both distance metrics. . . . . . . . . . . . . . . 132

4.8 3D Mesh Models with Keypoints . . . . . . . . . . . . . 133

4.9 Keypoint repeatability of diffusion with discrete Laplacians when adding signal noise . . . . . . . . . . . . . . . . . . . . . 138

4.10 Keypoint repeatability of diffusion with discrete Laplacians when adding vertex noise . . . . . . . . . . . . .

4.11 Keypoint repeatability of diffusion with discrete Gaussian when adding signal noise . . . . . . . . . . . . . . . . . . 143

4.12 Keypoint repeatability of diffusion with discrete Gaussian when adding vertex noise . . . . . . . . . . . . . . .

\section{Chapter 5. Feature Histogram Descriptors}

5.1 Reference frame for Itokawa. Azimuth and elevation angles are defined from the $x$-axis. Use this image to aid in reading Fig. 5.2.151 
5.2 Attitude (top) and position (bottom) errors produced from OURCVFH pose estimation. Blue is an accurate estimate with nearly no error. Position errors of 5 meters or more, and attitude errors of $20^{\circ}$ or more are shown as dark red. Azimuth and elevation angles are defined from the $x$-axis as shown in Fig. 5.1. (This figure is best viewed in color.) . . . . . . . .

5.3 Example OUR-CVFH clusters along simulated rendezvous trajectory with Itokawa. Each cluster is represented by a different color. If two clusters have the same color but are clearly separated, then they indicate different clusters. . . . . . . . .

5.4 Relative position error (left) and relative attitude error (right) for raw OUR-CVFH pose results during simulated rendezvous with Itokawa for all Monte Carlo runs. The varying observability as the viewpoint changes is evident in the growing and shrinking of the residuals. . . . . . . . . . . .

5.5 Relative position error (left) and relative velocity error (right) from the MEKF. The thin gray lines show results from individual Monte Carlo cases. The thick black lines show the $3 \sigma$ covariance bounds from the filter. . . . . . . . . . .

5.6 Relative attitude error (left) and relative body rate error (right) from the MEKF. The thin gray lines show results from individual Monte Carlo cases. The thick black lines show the $3 \sigma$ covariance bounds from the filter. . . . . . . . . . . . . .

5.7 Visual indications of modifications made to OUR-CVFH to develop SOUR-CVFH and SOL-CVFH . Section 5.2.1 discusses interpolation, Section 5.2.2 discusses description space, Section 5.2.4 discusses histogram normalization, Section 5.2.6 discusses cluster scale, and Section 5.2.9 discusses reference frame orientation. . . . . . . . . . . . .

5.8 Bin accumulation comparison for OUR-CVFH and the proposed LERP. The red dot is a value to be binned. In OUR-CVFH , the value completely lies within bin $b_{i}$. Using LERP, the value is distributed across bins $b_{i}$ and $b_{i+1}$ depending on the distance to the bin centers. . . . . . . . . . . . . . . .

5.9 Visual affects of LERP on histogram construction. . . . . . . . 168

5.10 Pose estimation errors for 500 run Monte Carlo experiment comparing OUR-CVFH to linear interpolation of angular distribution components. First row shows the box plots of the errors without outliers. Second and third rows show all pose estimation errors as a function of cost metric value. . . . . . . . . 
5.11 Spacing between inner-product space and angular space is not equal. (a) Equal spacing in angular space converted through $\cos (\cdot)$ to unequal spacing in inner-product space. (b) Equal spacing in inner-product space is converted through $\cos (\cdot)$ to unequal spacing in angular space. . . . . . . . . . . . .

5.12 Pose estimation errors for 500 run Monte Carlo for varying the description space of $\beta_{i}$. Method A describes $\beta_{i}$ in angular space, Method $\mathrm{B}$ describes $\beta_{i}$ in angular space and applies LERP to all angular distribution features. The first row shows box plots of the pose estimation errors without outliers. All errors as a function of cost metric value are shown in the second through fourth rows. . . . . . . . . . . . . . .

5.13 Visual affects of description space $\beta_{i}$ on histogram appearance.

5.14 A sensor view of an object. Inner product between surface normal vectors $\mathbf{n}_{i}$ and reverse camera boresight $\mathbf{s}_{b}$ (shown in red) create the angle $\beta$. Only small values of $\beta$ are possible, as large values signify that the surface is pointing away from the sensor. 174

5.15 Pose estimation errors in CDF and verse cost metric values for various descriptor settings. Method $\mathrm{A}$ is OUR-CVFH using the cost metric of Eq. 5.13 and Method B describes $\beta_{i}$ in angular space, uses LERP for angular distribution components, and the cost metric of Eq. 5.13. . . . . . . . . . . . . . . . . . . 177

5.16 Normalization of histogram individually by component. . . . .

5.17 Pose estimation errors without outliers for 500 run Monte Carlo varying the distance metric. Distances used are Jaccard (JC), Kullback-Leibler (KL), Chi-Squared (CS), and Jensen-Shannon (JS). Results are compared to OUR-CVFH . . . . . . . . .

5.18 All pose estimation errors as a function of cost metric value for 500 run Monte Carlo varying the distance metric. Distances used are Jaccard (JC), Kullback-Leibler (KL), Chi-Squared (CS), and Jensen-Shannon (JS). Compare to the OUR-CVFH results in first row of Fig. 5.10. . . . . . . . . . . . . . .

5.18 Continued. . . . . . . . . . . . . . . . 186

5.19 Model Itokawa with scene shown in yellow, clusters shown in blue, and the cluster's bounding circle defined by the scale shown in red. Only 2D circles are shown for clarity. . . . . . .

5.20 Pose estimation errors for 100 run Monte Carlo experiment for various scale thresholds using $\mathrm{JC}$ distance. . . . . . . . . .

5.21 All pose estimation errors as a function of cost metric value for 500 run Monte Carlo varying scale threshold $t_{\mathrm{r}_{\sigma}}$ using distance metric JC and requiring one axis scale ratio to meet threshold. 
5.22 All pose estimation errors as a function of cost metric value for 500 run Monte Carlo varying scale threshold $t_{\mathrm{r}_{\sigma}}$ using distance metric JC and requiring both axes scale ratios to meet threshold.195

5.23 Pose estimation errors for 100 run Monte Carlo experiment for various scale thresholds using CS distance. . . . . . . . .

5.24 All pose estimation errors as a function of cost metric value for 500 run Monte Carlo varying scale threshold $t_{r_{\sigma}}$ using distance metric CS and requiring one axis scale ratio to meet threshold..

5.25 All pose estimation errors as a function of cost metric value for 500 run Monte Carlo varying scale threshold $t_{\mathrm{r}_{\sigma}}$ using distance metric CS and requiring both axes scale ratios to meet threshold.199

5.26 Normalized histograms with various settings of ISOC weighting. 202

5.27 Pose estimation errors without outliers for 500 run Monte Carlo experiment various ISOC weighting using metric JC. Methods $\mathrm{A}, \mathrm{B}$, and $\mathrm{C}$ use all the modifications up to this point. Method A uses $\varsigma=0.01$, method B uses $\varsigma=\epsilon$, method $\mathrm{C}$ uses $\varsigma_{i}=\sqrt{\lambda_{i}}$, and method D uses LERP. . . . . . . . . . . . . . .

5.28 All pose estimation errors as a function of cost metric value for 500 run Monte Carlo varying ISOC weighting using metric JC. Methods A, B, C, and D use all the modifications up to this point. Method $\mathrm{A}$ uses $\varsigma=0.01$, method $\mathrm{B}$ uses $\varsigma=\epsilon$, method C uses $\varsigma_{i}=\sqrt{\lambda_{i}}$, and method D uses LERP. . . . . . . . . . 206

5.28 Continued . . . . . . . . . . . . . . . . .

5.29 Pose estimation errors without outliers for 500 run Monte Carlo experiment various ISOC weighting and varying resolution using metric JC. Methods A, B, and C use all the modifications up to this point. Method A uses $\varsigma=0.01$, method B uses $\varsigma=\epsilon$, method C uses $\varsigma_{i}=\sqrt{\lambda_{i}}$, and method D uses LERP. . . . . .

5.30 All pose estimation errors as a function of cost metric value for 500 run Monte Carlo varying ISOC weighting and varying resolution using metric JC. Methods A, B, and C use all the modifications up to this point. Method A uses $\varsigma=0.01$, method B uses $\varsigma=\epsilon$, method C uses $\varsigma_{i}=\sqrt{\lambda_{i}}$, and method D uses LERP. Vertical dashed line signifies the cost metric threshold of $t_{J}=0.4 \ldots \ldots \ldots \ldots \ldots$

5.30 Continued ...................... . . 212

5.31 Pose estimation errors for 500 run Monte Carlo experiment for various histogram component combinations using JC distance. 
5.32 All pose estimation errors as a function of cost metric value for the 500 run Monte Carlo varying histogram components for select methods A, C, D, G, I, and U. If the method includes scale, then both scale ratios meet the threshold, $l_{\sigma}=0.9$, and all use distance metric JC. . . . . . . . . . . . . . .

5.32 Continued. . . . . . . . . . . . . . . . . .

5.33 Pose estimation errors without outliers for 500 run Monte Carlo experiment various ISOC weighting using metric JC and LRF. Methods A, B, and C use all the modifications up to this point. SOL-CVFH uses a LRF and constructs ISOC with LERP. Cost metric threshold set at $t_{J}=0.5 \ldots \ldots$. . . . . .

5.34 All pose estimation errors as a function of cost metric value for 500 run Monte Carlo varying ISOC weighting using metric JC and LRF. SOL-CVFH uses a LRF and constructs ISOC with LERP. Vertical black dotted line is cost metric threshold set at $t_{J}=0.5 \ldots \ldots \ldots \ldots \ldots \ldots$

5.35 Visual affects of histogram appearance with SGURF or LRF construction. . . . . . . . . . . . . . 226

5.36 SOUR-CVFH cluster and histogram example. . . . . . . . . . 239

5.37 Example locations and ranges for database training procedure. The camera is placed at the vertices of a polyhedron oriented towards the object at multiple ranges. . . . . . . . . . .

\section{Chapter 6. Feature Histogram Performance Assessment}

6.1 3D Mesh Models for SOUR-CVFH testing. . . . . . . . . . . 251

6.2 Pose estimation results of 500 run Monte Carlo comparing OURCVFH to SOUR-CVFH for the model Armadillo. . . . . . . .

6.3 Pose estimation results of 500 run Monte Carlo comparing OURCVFH to SOUR-CVFH for the model Buddha. . . . . . . . . 254

6.4 Pose estimation results of 500 run Monte Carlo comparing OURCVFH to SOUR-CVFH for the model Bunny. . . . . . . . . .

6.5 Pose estimation results of 500 run Monte Carlo comparing OURCVFH to SOUR-CVFH for the model Dragon. . . . . . . . . 256

6.6 Pose estimation results of 500 run Monte Carlo comparing OURCVFH to SOUR-CVFH for the model Itokawa. . . . . . . .

6.7 Number of histogram matches for all five models for OURCVFH , SOUR-CVFH, and SOL-CVFH . . . . . . . . 258

6.8 Models of the SHOT Dataset 3 [2] used for object recognition in cluttered scenes. Each model is found in at least one set. . . 
6.9 SOUR-CVFH clusters overlaid on models of the SHOT Dataset 3. . . . . . . . . . . . . . . . . 264

6.10 Selected scenes from the SHOT Dataset 3 [2] used for object recognition in cluttered scenes. . . . . . . . . . . .

265

6.11 SOUR-CVFH clusters overlaid on selected scenes from the SHOT Dataset 3. . . . . . . . . . . . . . . .

6.12 Angle and range errors in CDF plots for each descriptor separated by set for the SHOT Dataset 3. OUR-CVFH shown in blue, SOUR-CVFH shown in orange, and SOL-CVFH shown in purple.

268

6.13 All angle and range errors as a function of cost metric for each descriptor separated by set for the SHOT Dataset 3. SOURCVFH shown in orange and SOL-CVFH shown in purple. OURCVFH not shown because the cost metric is unnormalized and only succeeded in one instance. . . . . . . . . . . . . .

6.14 Number of histogram matches for each descriptor separated by set for the SHOT Dataset 3. OUR-CVFH shown in blue, SOURCVFH shown in orange, and SOL-CVFH shown in purple. . .

6.15 CDF plots of (left) Number of histogram matches and (right) number of pose estimates from OUR-CVFH , SOUR-CVFH , and SOL-CVFH for the simulated approach to Itokawa. . . . . 275

6.16 Unfiltered pose estimates from OUR-CVFH for the simulated approach to Itokawa. . . . . . . . . . . .

6.17 Unfiltered pose estimates from SOUR-CVFH for the simulated approach to Itokawa. . . . . . . . . . . . . . . . . 277

6.18 Unfiltered pose estimates from SOL-CVFH for the simulated approach to Itokawa. . . . . . . . . . . . .

6.19 MEKF filtered attitude (left) and position (right) estimates from OUR-CVFH for the simulated approach to Itokawa. . . .

6.19 Continued. MEKF filtered velocity (left) and attitude rate (right) for OUR-CVFH . . . . . . . . . . . .

6.20 MEKF filtered attitude (left) and position (right) estimates from SOUR-CVFH for the simulated approach to Itokawa. . .

6.20 Continued. MEKF filtered velocity (left) and attitude rate (right) for SOUR-CVFH . . . . . . . . . . . .

6.21 MEKF filtered attitude (left) and position (right) estimates from SOL-CVFH for the simulated approach to Itokawa. . . .

6.21 Continued. MEKF filtered velocity (left) and attitude rate

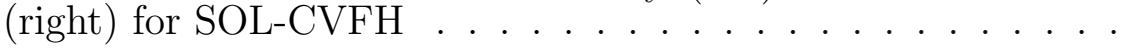




\section{List of Tables}

\section{Chapter 2. Review of Point-Cloud Feature Detection}

2.1 Scale-space construction methods on surfaces discussed in Sec. 2.1 compared to SIFT. The bottom two rows are the methods developed in this dissertation. . . . . . . . . . . . . . . 13

2.2 SOUR-CVFH evolution history. . . . . . . . . . . . 25

\section{Chapter 3. Signal Diffusion on 3D Meshes and Point-Clouds}

3.1 Four types of diffusion methods . . . . . . . . . . . . 63

3.2 LBO Convergence Type ................. . 81

\section{Chapter 5. Feature Histogram Descriptors}

5.1 Statistics for the 500 run Monte Carlo experiment comparing linear interpolation of angular distributions to OUR-CVFH . Method A uses LERP for $\cos \alpha_{i}, \cos \phi_{i}, \theta_{i}$, and $\cos \beta_{i}$. Method A performs better on every statistic except for Num. Pose Estimates and median angle error. . . . . . . . . . .

5.2 Statistics for the 500 run Monte Carlo experiment varying the description space of $\beta_{i}$ and linear interpolation to OUR-CVFH . Method $\mathrm{A}$ describes $\beta_{i}$ in angular space, Method $\mathrm{B}$ describes $\beta_{i}$ in angular space and applies LERP to all angular distribution features.

5.3 Statistics for the 500 run Monte Carlo experiment varying the description space of $\beta_{i}$ and linear interpolation to OUR-CVFH . Method A describes $\beta_{i}$ in angular space, Method B describes $\beta_{i}$ in angular space and applies LERP to all angular distribution features. . . . . . . . . . . . . . .

5.4 Statistics for the 500 run Monte Carlo experiment varying distance metric. . . . . . . . . . . . . . . . .

5.5 Statistics for the 500 run Monte Carlo experiment scale ratio threshold using distance metric JC. . . . . . . . . . . . 
5.6 Statistics for the 500 run Monte Carlo experiment scale ratio threshold using distance metric CS. . . . . . . . . . . .

5.7 Statistics for the 500 run Monte Carlo experiment varying ISOC weighting using metric JC. Method A uses $\varsigma=0.01$, method B uses $\varsigma=\epsilon$, method $\mathrm{C}$ uses $\varsigma_{i}=\sqrt{\lambda_{i}}$, and method D uses LERP. 205

5.8 Statistics for the 500 run Monte Carlo experiment varying ISOC weighting and varying resolution using metric JC. Method A uses $\varsigma=0.01$, method $\mathrm{B}$ uses $\varsigma=\epsilon$, method $\mathrm{C}$ uses $\varsigma_{i}=\sqrt{\lambda_{i}}$, and method D uses LERP. . . . . . . . . . . . . . .

5.9 Various histogram component combinations tested with 500 run Monte Carlo using distance metric JC. . . . . . . . . . . . . . 215

5.9 Continued . . . . . . . . . . . . . . . . 216

5.9 Continued . . . . . . . . . . . . . . . . . 217

5.10 Statistics for the 500 run Monte Carlo experiment varying ISOC weighting using metric JC and LRF. SOL-CVFH uses a LRF and constructs ISOC with LERP. Cost metric threshold set at $t_{J}=0.5 \ldots \ldots \ldots \ldots \ldots \ldots$

5.11 Threshold variables, preset values, and their applicable equation for SOUR-CVFH . . . . . . . . . . . . .

\section{Chapter 6. Feature Histogram Performance Assessment}

6.1 Statistics for the 500 run Monte Carlo experiment comparing OUR-CVFH, SOUR-CVFH, and SOL-CVFH for five models.

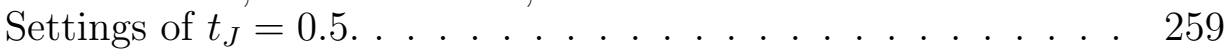

6.1 Continued. . . . . . . . . . . . . . . 260

6.1 Continued...................... 261

6.2 Statistics for the SHOT Dataset 3 experiment comparing OURCVFH, SOUR-CVFH, and SOL-CVFH for object recognition in cluttered scenes. Settings of $t_{\mathbf{n}}=\cos \left(3^{\circ}\right), t_{J}=0.1$, and only requiring one axis to meet the scale ratio threshold of $t_{\sigma}=0.9$. Numbers in parenthesis indicate the total amount possible. . . 271

6.3 Continued. . . . . . . . . . . . . . . . . 272 


\section{List of Publications}

Portions of this dissertation's content originally appeared in the conference and journal publications listed below.

- Rhodes, A. P. and Christian, J. A. , Scale-Space Construction and Keypoint Detection on 3D Meshes using Discrete Laplacians, Under Review by the International Journal of Computer Vision, 2019

- Rhodes, A. P. and Christian, J. A., Constructing a 3D Scale-Space from Implicit Surfaces for Vision-Based Spacecraft Relative Navigation, in AAS 41st Annual Guidance and Control Conference, February 2018

- Rhodes, A. P., Christian, J. A., and Robinson, S., Geometric Camera Calibration using Near-Field Images of the ISS Centerline Docking Plate, in AAS/AIAA Astrodynamics Specialist Conference, August 2017

- Rhodes, A. P., Christian, J. A., and Evans, T., A Concise Guide to Feature Histograms with Applications to Lidar-Based Spacecraft Relative Navigation, Journal of Astronautical Sciences, vol. 64, pp. 414-445, 2017

- Rhodes, A. P., Kim, E., and Christian, J. A., Lidar-Based Relative Navigation of Non-Cooperative Objects using Point Cloud Descriptors, in AIAA Space 2016, September 2016

- Jagat, A., Christian, J. A., and Rhodes, A. P., Vision-Based Relative Navigation using 3D Scale-Space Theory, in 26th AAS/AIAA Space Flight Mechanics Meeting, AAS 16-428, February 2016

- Flewelling, B., Murphy, T., Rhodes, A. P., Holzinger, M. J., and Christian, J. A., Spatio-Temporal Scale-Space Analysis of Photometric Signals with Tracking Error, in AMOS Technologies Conference, September 2015

- Sell, J., Rhodes, A. P., Woods, J. O., Christian, J. A., and Evans, T., Pose Performance of Lidar-Based Navigation for Satellite Servicing, in AIAA Astrodynamics Specialist Conference, August 2014

$\mathrm{XX}$ 


\section{Acknowledgments}

Thank you to my advisor Dr. John A. Christian for the many years guidance and education. The opportunity to join the Applied Space Exploration Laboratory (ASEL) under your direction has been an exciting and rewarding experience. Thank you for sticking with me through the many changes in research and institution.

Thank you to the members of my committee: Dr. Jason Gross, Dr. Yu Gu, Dr. Guilherme Pereira, and Dr. Stacie Williams. Thank you for your commitment in reviewing and offering feedback for this dissertation.

During my graduate studies, I had to fortunate experience of multiple internships. The first at Air Force Research Laboratory (AFRL) in Albuquerque, NM under the guidance of Dr. Brian Flewelling. Two summer tours at NASA Johnson Spaceflight Center (JSC) in Houston, TX under the guidance of Dr. Greg Holt and Dr. Chris D'Souza. The fourth at Autonomy Technology Research (ATR) Center and AFRL in Dayton, OH working with Dr. Sean O'Rourke and Dr. Clark Taylor. These mentors facilitated an exceptional learning environment and introduced me to the many availabilities of engineering applications.

I would like to thank a few of exceptional instructors that inspired me throughout my educational journey. Thank you to my high school physics in- 
structor Mike Roh. Your passion for physics, education, and hands-on student experience inspired me to pursue a path of engineering. Thank you my steel band ensemble instructor Dr. Michael Vercelli. Through 18 semesters in the steel drum ensemble you taught me the benefit of music in life and kept me motivated throughout my graduate program. Thank you to Dr. John Christian for noticing me as a senior undergraduate and sparking my interest in the world of computer vision and space navigation.

Thank you to my family. Thank you to my parents, Diane and Casey for the many years of support as I pursued my education. Thank you to my brother Alex for the fun years of brothership, shared music performances, and business ventures.

Thank you to my partner and wife Carissa. You saw me through the toughest years of my education and created a strong foundation for me to explore. Thank you for being my biggest champion. Thank you for always listening to my (occasionally) circuitous explanations of research. 


\section{Chapter 1}

\section{Introduction}

Many modern navigation applications include three-dimensional (3D) sensors for assistance in object recognition, position and attitude (pose) estimation, and map building. These processes require the alignment of two or more point-clouds measured by the $3 \mathrm{D}$ sensor, which is often accomplished using variants of the iterative closest point (ICP) algorithm. ICP initialization is particularly difficult, but generally must be sufficiently accurate for correct convergence. Correlation between the navigation filter and ICP initialization is avoided by using independent methods of pose estimation. The most common methods detect keypoints, succinctly describe features within a region of support, and estimate pose through matching descriptors.

The work presented in this dissertation investigates the use of scale to aid in the processes of keypoint detection, feature description, and matching. Scale - or size - is a characteristic of any keypoint or feature that describes the region of support. Features may include information on geometric attributes, color, or scale. Geometric information is an established component of 3D point-cloud descriptors; recent work has included color, but most only implicitly consider (if not completely disregard) information offered by scale. Scale 
is not a unique identifier alone, but assists in improving descriptor robustness and may quicken the matching procedure. Feature descriptors combining information from multiple data sources will be more robust to operating in various generalized environments. This work focuses on the exploitation of scale to develop new frameworks for keypoint detection, and entwining it into existing methods of feature description.

Work presented in this dissertation is motivated by - and lies at the intersection of - the success of $2 \mathrm{D}$ scale-space feature detectors and the recent ubiquitous use of 3D sensors. Scale-space is a process by which measurements (e.g. 1D signal, 2D images, 3D point-clouds) are analyzed at various levels of diffusion to identify multiple keypoints, each with their inherent characteristic scale. We adapt popular 2D scale-space techniques for diffusion of signals defined on 3D surfaces. The diffusion kernel merges and distributes information locally to simulate measurements at different ranges or resolutions. While 2D image diffusion commonly uses a discrete Gaussian, this work investigates the use of both the Gaussian and the Laplace Betrami operator (LBO) for 3D surface diffusion. Scale-space levels are conventionally separated by a constant ratio. The proposed scale-space using LBOs permits arbitrarily sized steps provided that specific scale-space levels are calculated. Scale-space construction using a Gaussian maintains equally spaced steps where the quantity of steps between levels increases exponentially. These construction styles are unique because the diffusion kernel is calculated only once - reducing one of the most computationally expensive processes. While most keypoint detection 
algorithms concurrently develop a companion descriptor, this work does not because the abundance of existing descriptors offers opportunity for finding an optimal pairing [3].

The work of this dissertation remodels a popular feature descriptor offering simultaneous object recognition and 6DOF pose estimation to explicitly utilize scale. A measured point-cloud is separated into multiple continuous clusters. For each cluster, one spatial and four angular features are calculated and concatenated into a normalized histogram. Each feature is linearly interpolated among neighbors to improve robustness to noise and processing errors. Stable local reference frames are constructed on each cluster to facilitate 6DOF pose estimation. Two versions of the descriptor are presented that differ only by their method of reference frame disambiguation. During the matching procedure, scale is first compared to determine the physical likeness between two clusters. Provided similar scales are present, the procedure proceeds to matching histograms. Relative pose is estimated using the cluster reference frames, and updated using ICP to maximize point-cloud overlap. The fundamental premise of scale accelerates the matching procedure and improves pose estimation accuracy by eliminating improbable cluster matches.

\subsection{Motivation}

\subsubsection{Sensor Development and Support}

Three dimensional (3D) sensors — such as time-of-flight cameras [4, 5], structured light sensors [5, 6], LIDARs [7], stereoscopic systems, and spacetime 
stereo $[8,9]$ — recently have become inexpensive and ubiquitous. All of these sensing systems produce a 3D point-cloud (sometimes referred to as a range image) of an observed scene. Emergence of such sensors has led to techniques for the processing, recognition, and registration of 3D models and point-clouds summarized by two enabling capabilities. First, robust and efficient pipelines for processing 3D point-clouds are now widely available and may be found in MATLAB, the open-source point-cloud Library (PCL) [10], and a variety of other software packages $[11,12,13]$. Second, mature techniques now exist for both recognizing and aligning objects in an observed 3D point-cloud with known 3D models of those same objects. A variety of 3D feature descriptors exist $[14,15,16]$ for applications spanning home entertainment [6], precision agriculture [17], industrial and home robotics [18], space exploration [7, 19, 16], and autonomous vehicle navigation [20].

Concurrent to terrestrial (mostly non-aerospace) applications previously listed, 3D sensors have also experienced a growing popularity in the spacecraft relative navigation (RelNav) community [7]. Such sensors address the well-known scale ambiguity associated with angles-only RelNav (e.g. the typical scenario with a regular 2D camera) [21] and are considered an enabling technology for many future missions with RelNav requirements [22]. These future RelNav scenarios can generally be divided into two classes: cooperative and non-cooperative. Cooperative RelNav describes the situation where the observed object is equipped with purpose-built navigation aids, such as reflectors for LIDARs [23, 24]. Non-cooperative RelNav describes the situation 
where navigation must be performed using the natural features of an object.

\subsubsection{Uncorrelated Pose Estimates in Navigation Filters}

Recent literature contains a variety of approaches for performing spacecraft RelNav using 3D point-clouds [22, 25, 26, 27, 19, 28, 29, 30, 16]. Most methods use the Iterative Closest Point (ICP) algorithm [31] or variant thereof $[32,33,34,35]$ to align the sensed point-cloud with a known model. Most ICP algorithms require a "good" pose initialization for correct convergence. Globally convergent ICP algorithms exist $[36,37]$, but do not operate in real-time and require parallelization. Consequently, the method and accuracy of ICP initialization for both initial acquisition and frame-to-frame tracking is of importance. Literature contains a variety of proposals for ICP initialization using methods such as polygonal aspect hashing [38], principal component analysis [39], template matching [28, 29], and feature histograms [25, 19, 30, 40, 16].

Frame-to-frame object tracking with ICP should be fed from independent pose estimates. Some have used the navigation filter's propagated pose as an initial input to ICP [41], but this is inherently dangerous for two reasons. First, a poor pose initialization may be propagated by the filter, by ICP, or both with no guarantee of recovery. Second, it introduces measurement errors that are correlated with a priori state errors [30]. This scenario for a sensor that estimates pose is shown in Fig. 1.1 with an external feedback loop from the output states to the sensor. This may be acceptable for particular states (such as sensor biases), but may introduce undesired errors for other states 


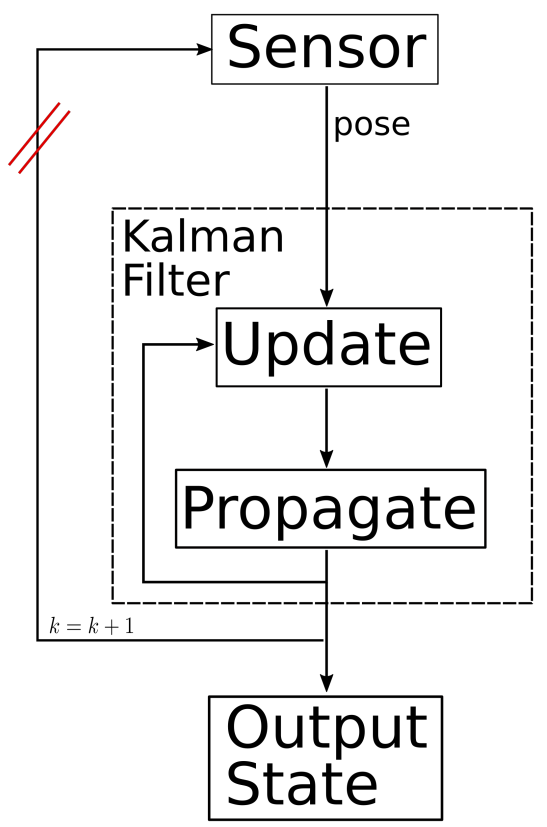

Figure 1.1: Example of information reuse by a Kalman Filter (KF) for a sensor that estimates pose. It is ill-advised to use the KF output state to influence the pose estimation, which is then ingested into the KF. To have independent and uncorrelated pose estimates, the link from output state to the sensor must be severed.

such as pose. To ensure the independence of filter states and ICP initialization, methods of pose estimation should be self-contained. Methods of independent pose estimation from point-clouds may also encounter - and therefore must be robust to - situations of clutter, occlusions, or missing data. Examples using uncorrelated measurements has been shown using a popular feature descriptor OUR-CVFH with real-time performance [30, 16]. 


\subsubsection{Scale-Space Feature Detection in 2D}

Scale-space theory has long been an area of interest in the computer vision community [42]. Scale-space is the mathematical process of examining data (images in this case) at various scales (e.g. size, resolution or distance). One outcome is the simultaneous detection of multiple features, each at their characteristic scale. An image may contain multiple features ranging in scale from a few pixels to the entire image size. Those same objects may appear differently depending on the scale at which they are measured. Scale-space feature identification improves robustness to affine projection, illumination, rotation, scale, clutter and occlusion. Research as shown that scale-spaces built with particular detectors appropriately model the retina receptor fields of the human visual system $[43,44]$.

Many scale-space construction and detection methods have been proposed in literature $[45,46,47,48,49,50]$ - of which the most popular are SIFT [49] and SURF [50] - leading to some generalized theories [51]. SIFT and SURF are prevalent in the computer vision community because of their speed and robustness. SIFT constructs a scale-space by sequentially convolving an discrete Gaussian with a repeatedly downsampled image. Keypoints are detected as local extrema in the difference of Gaussian (DoG) as an efficient approximation to the scale-normalized Laplace of Gaussian (LoG) [52]. Lo-

cal coordinate systems are constructed using the maximum orientation of local gradients. Gaussian weighted local gradients are accumulated into bins to construct the SIFT descriptor. SURF improves on computational speed by using 
box filters as simple approximations of the Laplacian. A scale-space is built by convolution of increasingly larger box filters with the integral image [53]. Keypoint detection is performed by analyzing the Hessian matrix determinant for local extrema. As with SIFT, keypoint localization is interpolated across space and scale. Keypoint support regions defined by their scale are convolved with Haar-wavelets to determine the orientation from local gradients. Haarwavelet responses summed across sub-regions of the keypoint support area to construct the SURF descriptor.

\subsection{Contributions}

This dissertation presents methods of utilizing scale in 3D feature detection and description. We present two distinct approaches to incorporating scale: the first from scale-space theory and the second by modifying a popular clustered viewpoint feature descriptor. This section discusses individually the contributions of this dissertation.

\subsubsection{Signal Diffusion on 3D Surfaces}

Identification and classification of signal diffusion methods is presented for a novel application of scale-space construction on 3D surfaces. Surface types of explicit and implicit representation are explored. Diffusion kernels of either the Gaussian or Laplace-Beltrami operator (LBO) are investigated. Combinations of surface representation and diffusion kernel leads to four categories of diffusion on 3D surfaces. We present the methodology of imple- 
menting each method and discuss their plausible use in terms of efficiency and accuracy. Equivalence to $2 \mathrm{D}$ signal diffusion on images is established visually followed by examples of signal diffusion on a variety of $3 \mathrm{D}$ models. This contribution is the focus of Chapter 3 .

\subsubsection{Scale-space Construction and Keypoint Detection}

New methods of scale-space construction for signals defined on 3D surfaces are developed. The well-established scale-space theory [42] is extended to explicit general 3D surfaces by adapting keypoint detection techniques from SIFT [49]. Either the Gaussian or LBO kernels are used to construct scalespace levels, where each level is separated by a constant ratio. Our discriminate selection of the ratio between levels permits the repeated use of a single diffusion kernel without requiring its reconstruction. A novel definition of the

local neighborhood based on distance assists to identify keypoints. Methods of non-maximum suppression of keypoints are specifically designed for use with LBOs. This contribution is the focus of Chapter 4.

\subsubsection{Scale in Clustered Viewpoint Feature Histograms}

Two new clustered viewpoint feature descriptors are created called Scaled Oriented Unique Repeatably Clustered Viewpoint Feature Histogram (SOUR-CVFH ) and Scaled and Oriented Locally CVFH (SOL-CVFH ). Their uniqueness comes from using either global or local information respectively to construct their reference frames. Their conception originates from the popular 
OUR-CVFH descriptor [54] followed by a series of alterations to improve pose estimation performance and reduce runtime. During reference frame construction, the cluster scales are simultaneously calculated. Scale similarity is utilized in the recognition framework to limit improbably cluster matches based on scale similarity. The effect is increased algorithm speed by performing fewer histogram comparisons. This contribution is the focus of Chapter 5 .

\subsubsection{Performance Assessments}

Performance assessments for scale, absolute, and relative repeatability are conducted for the scale-space keypoints (Chapter 4). Repeatability is tested on five 3D models using both of the diffusion kernels. Noise on both the signal and the vertex locations is analyzed. This work is included in Chapter 4.

Performance assessments for pose estimation accuracy and object recognition are conducted for SOUR-CVFH and SOL-CVFH (Chapter 5). The first scenario is a Monte Carlo experiment matching simulated range images of 3D models to a training database to test pose estimation accuracy. The second scenario matches a single range image to a cluttered scene to test pose estimation and object identification with occlusions. A third scenario simulates an autonomous approach to an asteroid in heliocentric orbit using rectilinear dynamics. These assessments are contained in Chapter 6 . 


\section{Chapter 2}

\section{Review of Point-Cloud Feature Detection}

\subsection{Keypoint Detection in Scale-Space}

Scale-space keypoint detection is an over-arching concept of identifying and describing objects at their natural scale - or size. The process is colloquially interchangeable with SIFT [49], but actually encompasses a larger framework of various kernels and detectors [51]. Scale-space constructed from a rigorous set of axioms dates back to the 1960's Iijima [55] and 1980's Otsu [56]. Interest in applications to imagery and computer vision abounded in the 1990's [52, 42]. Research in the early 2000's discovered that the human visual system may be modeled with Gaussian diffusion and the Difference of Gaussian (DoG) detector, leading to a biologically inspired scale-space [44]. Development in the 2010's of inexpensive and robust 3D sensors [7] has motivated the extension of scale-space theory and applications to 3D surfaces.

In our discussions, the purpose of scale-space applications in computer vision is to simultaneously identify multiple items (e.g. keypoints, features, objects) in a scene at their respective - and inherent - scales. SIFT successfully achieved this task using image intensity and became one of the most

widely used algorithms. Developing scale-space applications on 3D surfaces has 
lead to the diffusion of geometric quantities like mean curvature $[57,58,59]$ and normal vectors [60, 61], sensor intensities [62], physical point locations [63, 64, 65], arbitrary surface signal $[59,66,67]$ and a mixture of range and intensity data [68]. This section presents an overview of various methods that are most aligned to our proposed scale-space. These methods are summarized in Table 2.1 and compared to SIFT. Columns Method, Operator, and Item Diffused give the method name, diffusion kernel used, and over what attribute the scale-space is constructed respectively. Column Level Selection is how the method selects the separation of the scale-space levels. Column Preset Values shows method dependent settings for calculating Level Selection. Column Vertex Downsample details if the mesh is downsampled one or more times during scale-space construction, which also always corresponds to reconstruction of the diffusion kernel.

\subsubsection{Physical Scale Keypoints (PSK)}

Smith et al. combine information from 2D image intensity and range measurements to construct Physical Scale Keypoints (PSK) [68]. Grayscale image intensities are backprojected onto the Lidar range measurement, where diffusion is performed on the intensities while using point-cloud characteristics to select physical scales. It is robust to (1) self-occlusions from changing viewpoints and (2) changes in illumination direction because of their diffusion kernel selection. 
Table 2.1: Scale-space construction methods on surfaces discussed in Sec. 2.1 compared to SIFT. The bottom two rows are the methods developed in this dissertation.

\begin{tabular}{|c|c|c|c|c|c|}
\hline Method & Operator & Item Diffused & Level Selection & Preset Values & $\begin{array}{l}\text { Vertex } \\
\text { Downsample }\end{array}$ \\
\hline $\mathrm{SIFT}[49]$ & Gaussian & image intensity & $\sigma_{0} 2^{o+s / S}$ & $\begin{array}{l}S=3 \\
\sigma_{0}=1.6\end{array}$ & Yes \\
\hline GSS [69] & Gaussian & vertex signal & $\sigma_{\min }\left(\frac{\sigma_{\max }}{\sigma_{\min }}\right)^{n / 32}$ & $\begin{array}{l}\sigma_{\min }=\min e_{i j} / 2 \\
\sigma_{\max }=\max \sqrt{\sum \mathrm{A}\left(f_{i}\right)} / 5\end{array}$ & No \\
\hline HSS [67] & Gaussian & vertex signal & $\sigma_{0} 2^{o+s / S}$ & $S=3$ & Yes \\
\hline MeshSIFT [63] & Binomial & vertex locations & $\bar{e}\left(\sqrt{2 \sigma_{0} / 3}\right) 2^{n / k}$ & Unspecified & No \\
\hline ThrIFT [70] & Gaussian & density map & $\sigma_{0} k^{n}$ & Unspecified & Yes \\
\hline $\begin{array}{l}\text { MeshDog } \\
{[71,66]}\end{array}$ & Gaussian & vertex signal & $\begin{array}{l}\sigma_{0} \sqrt[3]{2} \\
\sigma_{0}(\sqrt[4]{2})^{\left\lceil\frac{n}{6}\right\rceil}\end{array}$ & $\sigma_{0}=\bar{e}$ & No \\
\hline $\mathrm{CS} 3[72,57]$ & Laplacian & mean curvature & $t_{0} k^{n}$ & $k>1$ & No \\
\hline $\begin{array}{l}\text { Method DE } \\
{[59], \text { Ch. } 3}\end{array}$ & Laplacian & vertex signal & $t_{0} k^{2 n}$ & $\begin{array}{l}k>1 \\
t_{0}=\bar{e} / 4\end{array}$ & No \\
\hline $\begin{array}{l}\text { Method GE } \\
\text { Ch. } 3\end{array}$ & Gaussian & vertex signal & $\sigma_{0}(\sqrt{2})^{n}$ & $\sigma_{0}=\bar{e} / 2$ & No \\
\hline
\end{tabular}


Diffusion is performed using a bilateral filter constructed of two Gaussians: one for inter-point Euclidean distances, and a second for normal vector inter-angles. The bilateral filter is

$$
\mathrm{B}\left(\mathbf{p}, \mathbf{n} ; \sigma_{\mathbf{p}}, \sigma_{\mathbf{n}}\right)=\exp \left(\frac{-\left\|\mathbf{p}_{i}-\mathbf{p}_{j}\right\|^{2}}{2 \sigma_{\mathbf{p}}^{2}}\right) \exp \left(\frac{-\left(1-\mathbf{n}_{i}^{\top} \mathbf{n}_{i}\right)^{2}}{2 \sigma_{\mathbf{n}}^{2}}\right)
$$

where $\sigma_{\mathbf{n}}=0.4$ but $\sigma_{\mathbf{p}}$ varies to construct the scale-space at multiple scales. Each level of diffusion is treated independently from another, i.e. each level is its own octave. Scales are selected relative to the measured point-cloud characteristics where the smallest scale $\sigma_{\min }$ equals the median average edge length. After each level of diffusion, the point-cloud is downsampled such that no two points lie within a distance $\sigma_{n} / 2$ of each other.

Keypoints are detected as extrema in the scale-normalized Laplace of Gaussian (LoG) within the first-ring neighborhood on a single level. The scalenormalized LoG is constructed as an indirect discretized LBO using gradients from both the intensity and the point-cloud. Since keypoints are extrema across space only, but not across scale, this multi-scale method detects more candidate keypoints than SIFT. Non-maximum suppression is applied to remove physically close repetitive keypoints within a radius of $3 \sigma_{n}$.

Each keypoint is given a support radius of $8 \sigma_{n} \sqrt{2}$ and a $3 \mathrm{D}$ coordinate frame defined by the keypoint normal vector and the dominate intensitygradient direction. Neighboring points within the support region are weighted using the bilateral filter with $\sigma_{\mathbf{p}}=\sigma_{n}$. Weights are accumulated into bins using partial volume interpolation to construct a 128 dimensional feature his- 
togram descriptor. K-d trees of histogram descriptors are constructed for each scale. Histogram matching is first distinguished by keypoint scale, followed by a Euclidean distance search using the scale specific k-d tree. Matching histograms only if the keypoints have the same scale reduces the search space and runtime. Keypoint coordinate frames are used to estimate the rigid transformation between scenes. Then a region-growing ICP, constrained by intensity and range, refines the rigid transformation estimate provided by the set of rank-ordered keypoint matches [73].

\subsubsection{Geodesic Scale-Space (GSS)}

Zou et al. construct a geodesic scale-space (GSS) using a Gaussian kernel with geodesic distances to diffuse a signal defined on surfaces [69]. The Gaussian kernel is constructed on the surface with a support window of twice the standard deviation. ${ }^{1}$ Since integration of a Gaussian with geodesic distances on a curved domain may be larger than unity, the kernel is renormalized to unity.

A scale-space is constructed on 32 logarithmically spaced levels where the scales are selected relative to the measured point cloud characteristics. The scales are

$$
\sigma_{n}=\sigma_{\min }\left(\frac{\sigma_{\max }}{\sigma_{\min }}\right)^{n / 32}
$$

\footnotetext{
${ }^{1} \mathrm{~A}$ support window of $2 \sigma$ is unusually small for a Gaussian. Usually they are at least $3 \sigma$ on $2 \mathrm{D}$ data and larger for higher dimensional data.
} 
where

$$
\begin{aligned}
\sigma_{\min } & =\min _{e_{i j} \in \mathcal{E}} e_{i j} / 2 \\
\sigma_{\max } & =\frac{1}{5} \sqrt{\sum_{f_{i} \in \mathcal{F}} \mathrm{A}\left(f_{i}\right)}
\end{aligned}
$$

and the Gaussian is reconstructed for each level of the scale-space. Consecutive levels of the scale-space are differenced to construct the DoG as an approximation to the scale normalized LoG. Keypoints are identified as extrema in space using first-ring neighborhood and across scale using three neighboring levels. Each keypoint is transformed to the origin and the first-ring neighborhood is projected to keypoint tangent plane. On the tangent plane, the SIFT non-maximum suppression process is used to eliminate keypoints of low contrast or high curvature. Keypoint localization is refined by fitting a quadratic function to the projected points and three neighboring scales to approximate the continuous DoG. Optimal selections of the quadratic function for keypoint scale and 2D location on the tangent plane are projected back to the 3D mesh.

GSS applies Spin Images [74] as the feature descriptor for the scalespace keypoints and the neighborhood points defined by the automatically selected scale. Feature matching follows the same procedure as Spin Images, but with reduced complexity because fewer keypoints are utilized. Keypoints and feature descriptors are used for applications of face matching. 


\subsubsection{Hierarchical Scale-Space (HSS)}

Hou and Qin extend the work of GSS to construct a hierarchical scalespace (HSS) for the purpose of detecting scale-space features on deformable surfaces [67]. A geodesic Gaussian kernel with a support window of twice the standard deviation ${ }^{1}$ diffuses a signal defined on the surface. Since integration of a Gaussian with geodesic distances on a curved domain may be larger than unity, the kernel is renormalized to unity.

HSS recognizes that geodesic distance is expensive to calculate on surfaces when the scale grows large. The scale-space is separated into octaves where each octave is initiated with a downsampled version of the original mesh. The mesh is simplified using uniform downsampling such that the number of faces is one-fourth that of the previous octave. Each octave is separated into $S=3$ levels where the scale is selected as

$$
\sigma_{n}=\sigma_{0} 2^{(o+s / S)}
$$

and the Gaussian is reconstructed for each level of the scale-space.

Consecutive levels in a octave are differenced to construct the DoG, in which keypoints are identified as extrema across space and scale. Keypoints and their neighborhoods are projected to the local tangent plane where the SIFT non-maximum suppression process is used to eliminate keypoints of low contrast or high curvature. Keypoint location is refined by fitting and evaluating a quadratic function to the projected points scales. Optimal selections 
of the quadratic function for keypoint scale and 2D location on the tangent plane are projected back to the 3D surface.

HSS uses a feature descriptor similar to that of gradient location and orientation histogram (GLOH) [75]. Keypoints and their neighborhood defined by the automatically selected scale are projected to the local tangent plane. A local coordinate system is created using the keypoint surface normal vector and the direction of maximum signal gradient. Geodesic distances from the keypoint to all points in the projected neighborhood separated by a polar grid of nine bins. Vertex counts are accumulated into the bins and vectorized to form a histogram suitable for matching. Keypoints and feature descriptors are used for applications of face matching.

\subsubsection{Mesh SIFT}

Smeets et al. construct a scale-space by diffusing vertex locations to create MeshSIFT [63]. This scale-space is unique from the others discussed in this section and summarized in Table 2.1 because the diffusion of vertex locations changes their position and the signal is reconstructed at each level. MeshSIFT approximates the Gaussian kernel by using a binomial filter such that the vertex locations are moved at each level according to

$$
\mathbf{v}_{i}^{(n+1)}=\frac{1}{\left|\mathcal{N}_{i}\right|}\left(\mathbf{v}_{i}^{(n)}+\sum_{j \in \mathcal{N}_{i}} \mathbf{v}_{j}^{(n)}\right)
$$

using the first-ring neighborhood $\mathcal{N}_{i}$. The number of levels is user specified, and there are no octaves. The exponential behavior of scales in SIFT are 
approximated using

$$
\sigma_{n}=\bar{e} \sqrt{\frac{2 \sigma_{0}}{3}} 2^{n / k}
$$

On each scale-space level, the mean curvature (of other signal of choice) is recalculated for each point using that level's scale. Consecutive levels of the scale-space are differenced to approximate the DoG where keypoints are identified as signal extrema in space using the first-ring neighborhood and across scale using three neighboring levels. The keypoint and its neighborhood defined by the automatically selected scale are projected to the local tangent plane. A local coordinate system is constructed using the keypoint normal vector and the direction of maximum signal gradient.

MeshSIFT develops its own feature descriptor. Using the keypoint canonical orientation, the circle of radius equal to the keypoint scale is separated into nine smaller overlapping circular regions. In each region, two features histograms are calculated for each point: (1) the shape index and (2) the normal vector inter-angle. Each histogram is given eight bins and is concatenated to make a single feature vector of length 144. Feature matching is performed by minimizing the inner-product of feature vectors using an angle

similarity measure. Keypoints and feature vectors are used for applications of face matching.

\subsubsection{ThrIFT}

Flint et al. adapt the SURF [50] detector and the SIFT [49] descriptor to $3 \mathrm{D}$ range data to create ThrIFT [70]. ThrIFT constructs a density map 
of the measured point cloud synonymous to pixel intensities in $2 \mathrm{D}$ images. The measured scene is discretized into isotropic voxels where each voxel may contain between zero and multiple points. Then the density function $f(x, y, z)$ is normalized by the maximum density voxel such that it has the range between 0 and 1.

A scale-space is constructed on the density map consecutive convolutions with a 3D Gaussian kernel using Euclidean distances. Scales for the scale-space are separated by a constant factor

$$
\sigma_{n+1}=k \sigma_{n}
$$

where the Gaussian kernel is reconstructed for each level (and scale). When the scale reaches two, the density map is downsampled by a factor of two. This downsampling reduces the data size as well as the Gaussian variance. For their experiments, the authors used $O=5$ octaves, each with $s=4$ levels.

Each level of the scale-space is convolved with the Gaussian second derivative to calculate the LoG. A 3D Hessian matrix is constructed using the second partial derivatives. Large values of the Hessian determinant identifies keypoints in space and scale. A non-maximum suppression technique is applied to eliminate keypoints with Hessian determinant values less than a threshold and compared to the nearest $3 \times 3 \times 3 \times 3$ voxels.

A keypoint's support neighborhood consists of all points lying within a sphere of radius equal to the automatically identified scale. For each point in the neighborhood, two normal vectors are calculated from the least squares 
plane fit: one each for a small and large support window. The inner-angle of the two normal vectors is calculated for each point and accumulated into histogram bins describing $0^{\circ}$ to $90^{\circ}$. The feature descriptor is normalized to unity.

\subsubsection{Mesh Difference of Gaussian (MeshDoG)}

Zaharescu et al. develop a scale-space for a scalar signal defined on a mesh using a geodesic Gaussian kernel and detect keypoints in the DoG (MeshDoG) $[71,66]$. Construction of the geodesic Gaussian kernel and the feature support region is defined by a novel distance-ring neighborhood. Contrary to the first-ring neighborhood used most 3D mesh processing algorithms, the distance-ring neighborhood consists of all vertices that lie within a geodesic sphere. This neighborhood definition is robust to arbitrary mesh constructing and non-uniform sampling because it is defined by distance instead of connectivity. Convolution with the geodesic Gaussian kernel is normalized by the kernel sum to maintain unity.

MeshDoG constructs a scale-space on scalar signals over $O=3$ octaves, with each octave containing $s=6$ levels. Their scale-space maintains constant mesh geometry throughout because mesh downsampling is a computationally expensive process. The standard deviation of the Gaussian kernel is defined by

$$
\sigma_{n}=\bar{e}(\sqrt[4]{2})^{\left\lceil\frac{n}{6}\right\rceil}
$$

where $\sigma$ remains constant during each octave. The Gaussian kernel is recon- 
structed at the beginning of each octave corresponding to the new standard deviation. Consecutive levels of the scale-space are differenced to construct the DoG as an approximation to the scale-normalized LoG.

Keypoints are detected as local extrema in space using the first-ring neighborhood and in across scale using the current and adjacent scales. Only extrema that exhibit a magnitude within the top $5 \%$ are further considered. Then for each keypoint, select a pair of orthonormal vectors lying in the local tangent plane. Calculate directional derivatives by projecting the keypoint normal vector along the two orthonormal tangent vectors. A Hessian matrix is constructed using the directional derivatives and symmetry is enforced by projection onto the linear space of $2 \times 2$ symmetric matrices. Keypoints that exhibit corner characteristics such that the ratio of the Hessian eigenvectors are below a threshold are retained for description.

A local coordinate system is constructed over the distance-ring neighborhood from the keypoint normal vector and the direction of the polar histogram bin with the most dominant gradient magnitude. MeshDoG develops a new feature descriptor by projecting the gradient vectors onto the three planes defined by the local coordinate system. Each plane is divided into four polar segments, with each further separated into eight orientation segments. Point counts are accumulated into the bins in which they lie. The final feature descriptor concatenates each of the polar and orientation histograms for all three axes for a final length of 96. Feature histograms are matched using a Euclidean distance metric. 


\subsubsection{Curvature Scale-Space 3D (CS3)}

Fadaifard et al. construct a scale-space on 3D meshes for the mean curvature signal (CS3) [57, 72]. Their framework is unique from the other method discussed in this section because they discretize the diffusion equation in time and utilize a discrete Laplacian as the diffusion kernel. The diffusion equation is discretized with a backward-in-time Euler scheme and solved with Biconjugate Gradient method

$$
\mathbf{u}_{n+1}=\left(\mathbf{I}-t_{n} \mathbf{L}\right)^{-1} \mathbf{u}_{n}
$$

where the LBO $\mathbf{L}$ is the umbrella operator. ${ }^{2}$ The time steps are selected as

$$
t_{n}=t_{0} k^{n}
$$

where $k>1$. Independent of the discretized diffusion equation, CS3 fits a Gaussian in the least squares sense to the transfer function in the frequency domain. The scale is estimated in the frequency domain, but not converted back to the spatial domain.

Consecutive levels of the scale-space are differenced to construct the DoG. Then CS3 defines their own scale-normalized LoG (different from SIFT [49]) as

$$
\Delta_{\text {norm }} \mathbf{u}_{n}=\frac{2 t_{n}}{t_{n+1}-t_{n}}\left(\mathbf{u}_{n+1}-\mathbf{u}\right)
$$

\footnotetext{
${ }^{2}$ This construction is novel because, unlike the Gaussian kernel, the LBO has theoretical convergence on arbitrary 3D surfaces.
} 
Keypoints are identified as extrema in $\Delta_{\text {norm }} \mathbf{u}$ in space using the first-ring neighborhood and across scale using the current and adjacent levels. Keypoint repeatability is determined by the accuracy of their location and automatically selected scale for various noise levels.

Instead of using a feature histogram, CS3 uses the time history of the scale-invariant LoG values as a function of scale for the feature descriptor. The scale-invariant LoG curve is calculated as

$$
\Delta_{\mathrm{si}} \mathbf{u}_{n}=\frac{1}{\delta_{n}}\left(\Delta \mathbf{u}_{n}-\overline{\mathbf{u}}_{n}\right)
$$

where

$$
\begin{aligned}
\overline{\mathbf{u}}_{n} & =\left(\frac{1}{m_{\mathbf{p}}} \sum \Delta \mathbf{u}_{n}\right) \mathbf{1}_{m_{\mathbf{p}} \times 1} \\
\delta_{n} & =\frac{1}{\sqrt{m_{\mathbf{p}}}}\left\|\Delta \mathbf{u}_{n}-\overline{\mathbf{u}}_{n}\right\|
\end{aligned}
$$

Keypoints and the scale-invariant LoG curves are used for applications of face matching.

\section{$2.2 \quad$ Feature Histogram Descriptors}

This dissertation develops a new feature histogram descriptor that is derived from a well-known family of feature histogram descriptors listed in Tab. 2.2. An extended background is given here Sec. 2.2 for sufficient understanding of the feature histogram descriptor history. Some of the content was previously published in [16]. For this dissertation, notation and terminology has been updated to maintain consistency and clarity of this document. 
Table 2.2: A brief comparison of the various feature descriptors that evolved into the SOUR-CVFH descriptor.

\begin{tabular}{llllll}
\hline \hline $\begin{array}{l}\text { Feature } \\
\text { Name }\end{array}$ & $\begin{array}{l}\text { Year } \\
\text { Pub. }\end{array}$ & $\begin{array}{l}\text { Description } \\
\text { Type }\end{array}$ & $\begin{array}{l}\text { Number } \\
\text { of Bins }\end{array}$ & Pose & Scale \\
\hline PFH [76] & 2008 & Local & $\begin{array}{l}16 \\
(125 \text { PCL })\end{array}$ & N/A & N/A \\
FPFH [77] & 2009 & Local & 33 & N/A & N/A \\
VFH [78] & 2010 & Global & 263 & 5 DOF & implicit \\
& 2011 & Regional & $308+90$ & 6 DOF & implicit \\
CVFH [79] & 2012 & Regional & 303 & 6 DOF & implicit \\
OUR-CVFH [54] & 2019 & Regional & $303+2$ & 6 DOF & explicit \\
SOUR-CVFH Sec. 5.3 & 2019 & Regional & $303+2$ & 6 DOF & explicit \\
SOL-CVFH Sec. 5.4 & 208 PCL & & &
\end{tabular}

\subsubsection{Persistent Point Feature Histogram (PFH)}

The first histogram proposed was the Persistent Point Feature Histogram (PFH ) by Rusu et al. [76]. Using point normals and curvature is a common practice in range image registration, but when used individually do not fully represent the surface. By better describing the geometric properties of a surface surrounding a point, the authors' objective was to improve the process of point correspondence. The PFH is a pose invariant feature histogram of values that describe the geometric properties of a local surface. This descriptor is also scale invariant, though the knowledge of scale is not as essential for the applications presented by the authors $[80,18]$. The purpose of PFH is to provide an approximate registration between two consecutive range images. This preliminary alignment is used as the initial guess for Point-to-Plane Iterative 
Closest Point (ICP) [32].

A PFH histogram, denoted as $\mathcal{H}$, is created for every point of a measured point-cloud $\mathcal{P}=\left\{\mathbf{p}_{i}, \mathbf{n}_{i}\right\}_{i=1}^{m_{\mathbf{p}}}$, where $\mathbf{p}_{i}$ is a measured $3 \mathrm{D}$ point returned from the sensor, and $\mathbf{n}_{i}$ is the associated normal calculated by any one of a handful of techniques [81]. The histogram generation is as follows:

1. For every point in the 3D measured point-cloud, select one point as the central point $\mathbf{p}_{c}$ and only analyze the neighborhood of points within a radius $r$ ( $k$-neighborhood). Or, mathematically, define the neighborhood of $\mathbf{p}_{c}$ as

$$
\mathcal{N}_{\mathbf{p}_{c}}=\left\{\mathbf{p}_{i} \in \mathcal{P}:\left\|\mathbf{p}_{c}-\mathbf{p}_{j}\right\|<r\right\}
$$

2. For every pair of points, $\mathbf{p}_{i}$ and $\mathbf{p}_{j}$, in the neighborhood of $\mathbf{p}_{c}$, label one as the source point $\mathbf{p}_{s}$ and the other the target point $\mathbf{p}_{t}$. The source point is the one with the smaller angle between its associate normal and the line connecting $\mathbf{p}_{j}$ and $\mathbf{p}_{k}$. In order to not consider the same pair of points twice, the constraints $j \neq k$ and $j<k$ are enforced.

3. Construct a Darboux frame (a locally defined moving frame) $\mathrm{D}=(\mathbf{u}, \mathbf{v}, \mathbf{w})$ centered at $\mathbf{p}_{s}$ with basis vectors defined as

$$
\begin{aligned}
\mathbf{u} & =\mathbf{n}_{s} \\
\mathbf{v} & =\frac{\left(\mathbf{p}_{t}-\mathbf{p}_{s}\right) \times \mathbf{u}}{\left\|\left(\mathbf{p}_{t}-\mathbf{p}_{s}\right) \times \mathbf{u}\right\|} \\
\mathbf{w} & =\mathbf{u} \times \mathbf{v}
\end{aligned}
$$

An example of a Darboux frame may be seen in Fig. 2.2. 
4. The histogram $\mathcal{H}$ contains four features defined by PFH :

$$
\begin{aligned}
& f_{1}=\mathbf{v}^{\top} \mathbf{n}_{t} \\
& f_{2}=\left\|\mathbf{p}_{t}-\mathbf{p}_{s}\right\| \\
& f_{3}=\mathbf{u}^{\top}\left(\frac{\mathbf{p}_{t}-\mathbf{p}_{s}}{\mathbf{p}_{t}-\mathbf{p}_{s}}\right) \\
& f_{4}=\arctan \left(\frac{\mathbf{w}^{\top} \mathbf{n}_{t}}{\mathbf{u}^{\top} \mathbf{n}_{t}}\right)
\end{aligned}
$$

Features $f_{1}$ and $f_{3}$ are inner products of two unit vectors and thus have the range \pm 1 . As an arctangent, $f_{4}$ has the range $\pm \frac{\pi}{2}$. PFH separates each feature into two divisions. The division of features $f_{1}, f_{3}, f_{4}$ is the center of their intervals (i.e. 0), while the division for $f_{2}$ is the search radius $r$. The total number of combinations between the four features is $2^{4}=16$ bins, where each bin contains a percentage of the points that satisfy the criteria. Rusu et al. [76] decided on only two divisions because they desired to keep the descriptor as small as possible, and two divisions provided good results.

The reader can see that it would be too cumbersome to store and match the descriptor of every point in a scene, so PFH finds and saves only the features of persistent points. These are points that are seen as important at multiple scales. Persistent points are found as follows:

1. Find the histogram for every point in the scene over a range of radii $r_{i}, i=1, \ldots, m_{r}$, which is varied over an interval depending on the 
point-cloud size and density. Rusu et al. [76] use the range $2 \mathrm{~cm}<r<$ $3.5 \mathrm{~cm}$. While this range makes sense for personal robotics, a different range is necessary for many outdoor terrestrial applications or for space applications.

2. Then, the $m_{\mathbf{p}}$ points existing in $\mathcal{P}$ will each have $m_{r}$ feature histograms $\mathcal{H}^{\mathrm{f}_{i}}$. Take the average of all of these histograms to find the mean histogram $\mathcal{H}^{\mu}$ of the point-cloud.

3. For a certain point that has multiple feature histograms $\mathcal{H}^{\mathrm{f}_{i}}$ generated by varying $r_{i}$, match them all to the mean histogram $\mathcal{H}^{\mu}$ using the KullbackLeibler distance metric [82]

$$
\mathrm{d}_{\mathrm{KL}}=\sum_{j=1}^{16}\left(\mathcal{H}_{j}^{\mathrm{f}_{i}}-\mathcal{H}_{j}^{\mu}\right) \ln \left(\frac{\mathcal{H}_{j}^{\mathrm{f}_{i}}}{\mathcal{H}_{j}^{\mu}}\right)
$$

where $\mathcal{H}^{\mathrm{f}_{i}}$ is the feature histogram of a certain point at a radius $r_{i}$ and $\mathcal{H}^{\mu}$ is the mean histogram of the entire point-cloud.

4. A particular point is unique when it has a descriptor distance outside the interval $\mathcal{H}^{\mu} \pm \alpha \sigma$, where $\alpha$ controls the width of the interval that defines outliers and $\sigma$ is the standard deviation of the distance distribution. Rusu et al. set $\alpha=1$ resulting in about $10-20 \%$ unique features. A point is called persistent when it is unique in both $r_{i}$ and $r_{i+1}$, or

$$
P_{f}=\bigcup_{i=1}^{n-1}\left[P_{f_{i}} \cap P_{f_{i+1}}\right]
$$

where $P_{f_{i}}$ is the set of points that are unique for a given radius $r_{i}$. 
To prove that this set of features are suitable to distinguish different geometrical surfaces, Rusu et al. show the PFH of various surfaces. The PFH for a point on a sphere, plane, side of a cylinder, and on the edge of a cube all produce sufficiently distinct signatures in the feature histogram space.

To register to range images, $\mathrm{PFH}$ constructs corresponding pairs of persistent feature points. In addition to matching histograms between two range images, they apply geometric consistency constraints to ensure correct correspondences. Correspondence candidates are selected if they are equidistant from each other in both point-clouds. Now using the pairs, a transformation matrix is constructed to align the point-clouds. This initial estimate is then updated by using point-to-plane ICP.

While the initial publication dictates that the PFH has four features separated into two divisions each to create $2^{4}=16$ bins, its implementation in PCL is slightly different. PCL finds that $f_{2}$ rarely provides useful information and thus its calculation is ignored, leaving only three features. PCL then splits each of these features - $f_{1}, f_{3}$, and $f_{4}$ - into five divisions. Thus, the total number of combinations between these three features is $5^{3}=125$ bins. Because of the increased feature resolution (five divisions instead of two divisions), the PCL version of PFH histogram is more descriptive than in the publication.

The generation method of PFH histograms is computationally expensive because it considers every pair of points in the neighborhood of the query point $\mathbf{p}_{c}$. The computational complexity for $n$ query points, each with a $k$ neighborhood of points is $O\left(n k^{2}\right)$, which makes such an approach not suitable 
for real-time applications.

\subsubsection{Fast Point Feature Histogram (FPFH)}

Rusu et al. [77] created the Fast Point Feature Histogram (FPFH ) in order to make PFH suitable for real-time applications. The improvements introduced in FPFH allow it to operate in real-time but the FPFH descriptor is still pose and scale invariant. In brief, Rusu et al. supply two major improvements in going from $\mathrm{PFH}$ to $\mathrm{FPFH}$.

First, the range image dataset is reordered so that the points which are located physically close to one another have incremental index values. The intent here is to be able to cache computed feature values for later use. If two query points, $\mathbf{p}_{i}$ and $\mathbf{p}_{j}$, are in the neighborhood of each other, then many of their neighbor points will be shared. When the histogram of $\mathbf{p}_{i}$ is being computed, some features values will also be used when next computing the histogram of $\mathbf{p}_{j}$. Thus, the lookup of feature values from the cache is faster than recalculating those features. If the cache grows too large, they employ a first-in, first-out (FIFO) strategy.

Second, when calculating the features of a query point $\mathbf{p}_{c}$, FPFH does not consider every combination of all its neighbors. Only pairs between the query point and its neighbors are considered. Rusu et al. found that the feature $f_{2}$ of $\mathrm{PFH}$ that characterized the Euclidean distance between a pair of points supplied no significant benefit. They found that the robustness of the descriptor was not impeded when they forwent the calculation of $f_{2}=\| \mathbf{p}_{j}-$ 
$\mathbf{p}_{i} \|$. So the FPFH only calculates three features to include in the histogram. This conclusion is consistent with the decision to remove of $f_{2}$ from the PCL implementation of PFH (see Sec. 2.2.1).

A FPFH histogram is created for every point of a measured point-cloud $\mathcal{P}=\left\{\mathbf{p}_{i}, \mathbf{n}_{i}\right\}_{i=1}^{m_{\mathbf{p}}}$, where $\mathbf{p}_{i}$ is a $3 \mathrm{D}$ point returned by the sensor and $\mathbf{n}_{i}$ is the associated normal (a computed quantity). Only points within a radius $r$ of a central point $\mathbf{p}_{c}$ are included when generating the histogram. The histogram generation is as follows:

1. For every point in the 3D measured point-cloud, select one point the central point $\mathbf{p}_{c}$ and only analyze the neighborhood of points within a radius $r$ ( $k$-neighborhood). See Eq. 2.16 for details.

2. At the central point $\mathbf{p}_{c}$, place a Darboux frame $\mathbf{D}=(\mathbf{u}, \mathbf{v}, \mathbf{w})$ as

$$
\begin{aligned}
\mathbf{u} & =\mathbf{n}_{c} \\
\mathbf{v} & =\frac{\left(\mathbf{p}_{j}-\mathbf{p}_{c}\right) \times \mathbf{u}}{\left\|\left(\mathbf{p}_{j}-\mathbf{p}_{c}\right) \times \mathbf{u}\right\|} \\
\mathbf{w} & =\mathbf{u} \times \mathbf{v}
\end{aligned}
$$

where the subscript $j$ represents any point in the neighborhood of $\mathbf{p}_{c}$.

3. Redefine the features from $\mathrm{PFH}$ as

$$
\begin{aligned}
\cos \left(\alpha_{i}\right) & =f_{1}=\mathbf{v}^{\top} \mathbf{n}_{j} \\
\cos \left(\phi_{i}\right) & =f_{3}=\mathbf{u}^{\top}\left(\frac{\mathbf{p}_{i}-\mathbf{p}_{c}}{\left\|\mathbf{p}_{i}-\mathbf{p}_{c}\right\|}\right) \\
\theta_{i} & =f_{4}=\arctan \left(\frac{\mathbf{w}^{\top} \mathbf{n}_{i}}{\mathbf{u}^{\top} \mathbf{n}_{i}}\right)
\end{aligned}
$$


These feature values are only calculated between the central point and its $k$-neighbors. Rusu et al. also notice that due to the geometrical properties of a group of points, there are many bins in the $\mathrm{PFH}$ that have a value of zero. These empty bins are considered redundant, and are wasteful to have. Therefore, the features, $\cos (\alpha), \cos (\phi)$, and $\theta$ are concatenated to form what is now called the simplified point feature histogram (SPFH ). Notice that $f_{2}$ from PFH is not used here.

4. For each central point, use the $S P F H$ histograms of its $k$-neighbors to weight the final FPFH histogram of that central point $\mathbf{p}_{c}$ as

$$
F P F H\left(\mathbf{p}_{c}\right)=S P F H\left(\mathbf{p}_{c}\right)+\frac{1}{k} \sum_{i=1}^{k} \frac{1}{w_{i}} S P F H\left(\mathbf{p}_{i}\right)
$$

where $w_{i}=\mathbf{p}_{i}-\mathbf{p}_{c}$ is the Euclidean distance between the two points.

Once again, finding and storing the FPFH for every point in the range image would be a cumbersome task, so the authors find the persistent features with the same method as presented in [76].

The original publication does not state the number of bins for which each feature should be separated. The PCL implementation selects 11 divisions for each feature, making the and the FPFH histograms have 33 bins. Note that the total number of bins for $n$ features with $m$ divisions is $n m$ since the features are concatenated. This is more efficient than combining all the features into a single histogram (as was done with PFH ) which would result in $m^{n}$ bins. 
A sample consensus method for initial alignment (SAC-IA) is used to determine the relative pose between two range images of an object. A set of three points that are geometrically consistent with one another in the first point-cloud are selected. They are then matched to points with similar histograms in the second point-cloud. A rigid transformation is then found to align the point-clouds. They use a Huber penalty [83] to determine the quality of the transformation. After repeating for all combinations of three points, the transformation with the best Huber score is selected and refined using a Levenberg-Marquardt non-linear solver [84, 85].

Because the new formulation of the feature histogram does not consider every pair of points within the neighborhood of a central point, the FPFH is missing point pairs that may contribute to the geometry around the $\mathbf{p}_{c}$. The resulting algorithm does however decrease the operational complexity of $n$ points, each having a $k$-neighborhood, to $O(n k)$.

\subsubsection{Viewpoint Feature Histogram (VFH)}

Since the previous two histogram descriptors were invariant to an ob-

ject's scale and its pose, Rusu et al. [78] modified the construction style of PFH and FPFH to create the Viewpoint Feature Histogram (VFH ). Their goal was to create a descriptor that could be run in real time to simultaneously recognize an object and calculate its pose. VFH addresses the pose invariance of PFH and FPFH by considering the camera viewpoint as a fourth feature. This new feature is then concatenated to the established features from 
FPFH . This fourth feature is the distribution of angles between the viewpoint direction and each points' normal vector.

The most distinctive difference between $\mathrm{PFH}, \mathrm{FPFH}$, and VFH is the number of histograms generated per point-cloud. Recall that PFH and FPFH created a descriptor for each point in the point-cloud. VFH creates only one descriptor per scene point-cloud. The largest assumption is that the scene has been segmented so that each object is considered independently [86]. Therefore, each segmented scene contains only one object and will have only one descriptor.

A segmented scene will have a measured point-cloud $\mathcal{P}=\left\{\mathbf{p}_{i}, \mathbf{n}_{i}\right\}_{i=1}^{m_{\mathbf{p}}}$ where $\mathbf{p}_{i}$ is a $3 \mathrm{D}$ point returned by the sensor and $\mathbf{n}_{i}$ is the associated normal (a computed quantity). Every point in the scene is included when finding the histogram which is generated as follows:

1. For the scene point-cloud, find the centroid and average surface normal respectively as

$$
\begin{aligned}
& \overline{\mathbf{p}}=\frac{1}{m_{\mathbf{p}}} \sum_{i \in \mathcal{P}}^{m} \mathbf{p}_{i} \\
& \overline{\mathbf{n}}=\frac{\frac{1}{m_{\mathbf{p}}} \sum_{i=1}^{m_{\mathbf{p}}} \mathbf{n}_{i}}{\left\|\frac{1}{m_{\mathbf{p}}} \sum_{i=1}^{m_{\mathbf{p}}} \mathbf{n}_{i}\right\|}
\end{aligned}
$$


2. Define a Darboux frame $\mathrm{D}=(\mathbf{u}, \mathbf{v}, \mathbf{w})$ for each point $\mathbf{p}_{i}$ as

$$
\begin{aligned}
\mathbf{u}_{i} & =\overline{\mathbf{n}} \\
\mathbf{v}_{i} & =\frac{\left(\mathbf{p}_{i}-\overline{\mathbf{p}}\right) \times \mathbf{u}_{i}}{\left\|\left(\mathbf{p}_{i}-\overline{\mathbf{p}}\right) \times \mathbf{u}_{i}\right\|} \\
\mathbf{w}_{i} & =\mathbf{u}_{i} \times \mathbf{v}_{i}
\end{aligned}
$$

3. For every point in the segmented point-cloud, calculate the three features carried over from FPFH

$$
\begin{aligned}
\cos \left(\alpha_{i}\right) & =\mathbf{v}_{i}^{\top} \mathbf{n}_{i} \\
\cos \left(\phi_{i}\right) & =\mathbf{u}_{i}^{\top}\left(\frac{\mathbf{p}_{i}-\overline{\mathbf{p}}}{\mathbf{p}_{i}-\overline{\mathbf{p}}}\right) \\
\theta_{i} & =\arctan \left(\frac{\mathbf{w}_{i}^{\top} \mathbf{n}_{i}}{\mathbf{u}_{i}^{\top} \mathbf{n}_{i}}\right)
\end{aligned}
$$

4. Calculate the camera viewpoint feature (i.e. the new fourth feature) as

$$
\cos \left(\beta_{i}\right)=\mathbf{n}_{i}^{\top}\left(\frac{\mathbf{s}_{o}-\overline{\mathbf{p}}}{\left\|\mathbf{s}_{o}-\overline{\mathbf{p}}\right\|}\right)
$$

where the sensor origin is often assumes to be $\mathbf{s}_{o}=[0,0,0]^{\top}$.

Because of the additional component of the camera viewpoint feature $\cos (\beta)$ in the VFH histogram, this descriptor may now be used to identify objects in 3D space and estimate their relative poses. Rusu et al. [78] developed VFH for use in home robotics where a personal robot could correctly identify and select a desired object from a collection of objects. They assume that the robot will always be oriented upwards and never on its side or upside-down. 
As a result, the VFH descriptor does not consider — and is thus invariant to - camera roll.

$\mathrm{VFH}$ is more robust than $\mathrm{PFH}$ and FPFH in describing an object because it increases the number of divisions for each feature. The features $\cos \left(\alpha_{i}\right), \cos \left(\phi_{i}\right), \theta_{i}$ each contain 45 bins, while the new feature $\cos \left(\beta_{i}\right)$ has 128 bins. The resulting histograms are concatenated in the order $\mathcal{H}=[\cos (\alpha), \cos (\phi), \theta, \cos (\beta)]$ for a total VFH histogram size of 263 bins.

The PCL implementation attempts to be consistent with future modifications of the histogram style descriptor by reintroducing a variant of the $f_{2}$ feature from Sec. 2.2.1 that characterizes the Euclidean distances between points in a segmented surface. It is normalized by the Euclidean distance between the centroid to the farthest point in the segmented scene. The VFH histogram created in PCL is concatenated as $\mathcal{H}=\left[\cos (\alpha), \cos (\phi), \theta, f_{2}, \cos (\beta)\right]$ for a size of 308 bins.

To be able to recognize an object, it is necessary to have a training data set. Rusu et al. place a physical object on a tilting platform and take thousands of images from which VFH descriptors are generated. The objects are imaged in azimuth up to $180^{\circ}$, and in elevation up to $30^{\circ}$. This process is time consuming, but is necessary because they use stereo cameras with known epipolar geometry to triangulate the 3D scene. This cumbersome process of object feature training is addressed in later generations of this histogram style descriptor. Matching a feature histogram from the scene to the training dataset follows the same Huber penalty as FPFH (see Sec. 2.2.2). 
The authors do not address an object's scale in the VFH histogram because they assume that the dense stereo depth image explicitly provides distance and thus scale. The height of the VFH histogram implicitly relays information about the object's scale, as it is a function of the total number of points in the range image. In the PCL implementation, the histogram is normalized by the total number of points in the scene, which makes all histograms have the same height range of [0-1]. This inconsistency provides insight into the difficulty that these feature histograms have with relaying information on the scale of an object.

\subsubsection{Clustered Viewpoint Feature Histogram (CVFH)}

Aldoma et al. [79] were interested in recognizing an object at any arbitrary 6DOF pose when matched against a training set of 3D CAD models. They noticed that the features used in VFH were sufficiently descriptive, though still had limitations in characterizing an object's scale and estimating full 6DOF pose. The Clustered Viewpoint Feature Histogram CFVH addresses the deficiencies present in (VFH ) by (1) being able to distinguish identical objects of different scales and (2) considering the effects of partial occlusions and scene segmentation artifacts. The CVFH histogram is not normalized by the total number of points in the scene, so the object's scale is implicitly characterized by the histogram. The process of scene segmentation or partial occlusions may result in undesirable artifacts on the point-cloud. This prompted the authors of [79] to cluster the segmented scene so that descrip- 
tors are only created on the stable regions. However the assumption of a pre-processing scene segmentation still exists.

CVFH remedies the roll invariance of VFH by concatenating a camera roll histogram onto the descriptor. This roll histogram captures the angle between the camera viewpoint vector and the normals of the point-cloud points. In order to reduce the computational complexity, these camera roll histograms are only generated for the best matching views of an object.

Aldoma et al. also addressed the cumbersome training routine performed by VFH . Manually taking thousands of images of a variety of objects is time consuming and difficult, especially when trying to capture all viewpoints and poses. They instead use widely available 3D CAD models of everyday items around which they place a virtual 3D sensor to simulate point-clouds. This method greatly eases the cost of scaling the set of objects that a robot can recognize. Capturing all desired viewpoints of an object at various ranges is now a straightforward task.

Consider a measured point-cloud $\mathcal{P}=\left\{\mathbf{p}_{i}, \mathbf{n}_{i}, c_{i}\right\}_{i=1}^{m_{\mathbf{p}}}$ where $\mathbf{p}_{i}$ is a $3 \mathrm{D}$ point location, $\mathbf{n}_{i}$ is the associated normal, and $c_{i}$ is the associated curvature value. Note that $\mathbf{p}_{i}$ is a measurement returned by the sensor while $\mathbf{n}_{i}$ and $c_{i}$ are computed quantities. Generate the CVFH descriptor as follows:

1. First remove points from $\mathcal{P}$ that have a curvature larger than a threshold $t_{c}$. The resulting filtered point-cloud is labeled as $\mathcal{P}^{f}$. The removed points represent artifacts from sensor noise, object edges, or non-planar 
patches. Then the filtered point-cloud will be the set

$$
\mathcal{C}^{f}=\left\{\mathbf{p}_{i}, \mathbf{n}_{i}\right\}_{i=1}^{m_{\mathbf{p}}^{f}}
$$

where $m_{\mathbf{p}}^{f} \leq m_{\mathbf{p}}$.

2. Place a voxel grid (the $3 \mathrm{D}$ counterpart to the $2 \mathrm{D}$ pixel; the reader is referred to [87] for more information on voxels) over the measured pointcloud. This grid may be thought of as individual square boxes surrounding each 3D point. Each voxel contains only one point, though that point may not be centered in the box. Every box has identical size and no boxes overlap. Each voxel may be characterized by the single size parameter $\sigma$. Then, for each point, change its value to that of the centroid of the voxel in which it is contained. This process removes some noise characteristics from the measured point-cloud and smooths the data.

3. A smooth, continuous region growing algorithm separates the filtered point-cloud $\mathcal{P}^{f}$ into clusters $\mathcal{C}_{k}, k=1, \ldots, m_{\mathfrak{e}}$. Initiate a cluster with a random point and its normal vector from $\mathcal{P}^{f}$. Then a point belongs to that cluster if the dot product of the normals of two neighboring points is less than an angle threshold $t_{n}$ and if those same neighboring points are separated by less than an Euclidean distance threshold $t_{d}$.

$$
\mathcal{C}_{k}=\left\{\mathbf{p}_{i} \in \mathcal{P}^{f}:\left(\mathbf{p}_{i}-\mathbf{p}_{j} \leq t_{d}\right) \wedge\left(\mathbf{n}_{i}^{\top} \mathbf{n}_{j} \leq t_{n}\right)\right\}
$$

Aldoma et al. set $t_{d}=3 \sigma$, where $\sigma$ is the voxel grid size, and $t_{n}=\cos \left(10^{\circ}\right)$. The PCL implementation sets these as default values, but permits them to be modified by the user. 
4. Then a cluster will carry the defining set

$$
\mathcal{C}_{k}=\left\{\overline{\mathbf{p}}, \overline{\mathbf{n}},\left\{\mathbf{p}_{i}, \mathbf{n}_{i}\right\}_{i=1}^{m_{\mathbf{p}, k}}\right\}_{k=1}^{m_{\mathcal{C}}}
$$

where $m_{\mathbf{p}, k} \leq m_{\mathbf{p}}^{f}$, the cluster centroid is $\overline{\mathbf{p}}$, and the associated average normal vector is $\overline{\mathbf{n}}$ defined respectively as

$$
\begin{aligned}
\overline{\mathbf{p}} & =\frac{1}{m_{\mathbf{p}, k}} \sum_{i=1}^{m_{\mathbf{p}, k}} \mathbf{p}_{i} \\
\overline{\mathbf{n}} & =\frac{\frac{1}{m_{\mathbf{p}, k}} \sum_{i=1}^{m_{\mathbf{p}, k}} \mathbf{n}_{i}}{\left\|\frac{1}{m_{\mathbf{p}, k}} \sum_{i=1}^{m_{\mathbf{p}, k}} \mathbf{n}_{i}\right\|}
\end{aligned}
$$

After the segmented scene is separated into clusters, the remaining process is very similar to VFH . The only difference is that instead of one histogram for the entire scene, there is now a CVFH histogram descriptor for each cluster in the scene. Therefore, do the following for each cluster:

1. Define a Darboux frame $\mathrm{D}=(\mathbf{u}, \mathbf{v}, \mathbf{w})$ (see Fig. 2.2) for each point in a cluster. Here, the centroid and average normal are of the given cluster and not of the entire point-cloud.

$$
\begin{aligned}
\mathbf{u}_{i} & =\overline{\mathbf{n}} \\
\mathbf{v}_{i} & =\frac{\left(\mathbf{p}_{i}-\overline{\mathbf{p}}\right) \times \mathbf{u}_{i}}{\left\|\left(\mathbf{p}_{i}-\overline{\mathbf{p}}\right) \times \mathbf{u}_{i}\right\|} \\
\mathbf{w}_{i} & =\mathbf{u}_{i} \times \mathbf{v}_{i}
\end{aligned}
$$

2. For every point in the cluster, calculate the four features carried over 
from VFH

$$
\begin{aligned}
\cos \left(\alpha_{i}\right) & =\mathbf{v}_{i}^{\top} \mathbf{n}_{i} \\
\cos \left(\phi_{i}\right) & =\mathbf{u}_{i}^{\top}\left(\frac{\mathbf{p}_{i}-\overline{\mathbf{p}}}{\left\|\mathbf{p}_{i}-\overline{\mathbf{p}}\right\|}\right) \\
\theta_{i} & =\arctan \left(\frac{\mathbf{w}_{i}^{\top} \mathbf{n}_{i}}{\mathbf{u}_{i}^{\top} \mathbf{n}_{i}}\right) \\
\cos \left(\beta_{i}\right) & =\mathbf{n}_{i}^{\top}\left(\frac{\mathbf{s}_{o}-\overline{\mathbf{p}}}{\left\|\mathbf{s}_{o}-\overline{\mathbf{p}}\right\|}\right)
\end{aligned}
$$

3. Recall the $f_{2}$ feature in Sec. 2.2.1 that was dropped in subsequent publications. CVFH re-introduces a variant of this feature as the shape distribution component $(S D C)$. The $S D C$ is normalized by the distance between the centroid and the farthest point in the cluster as

$$
S D C_{i}=\frac{\overline{\mathbf{p}}-\mathbf{p}_{i}}{\max _{\mathbf{p}_{i} \in \mathcal{e}_{k}}\left\{\overline{\mathbf{p}}-\mathbf{p}_{i}\right\}}
$$

The contribution of the $S D C$ is in assisting to distinguishing between surfaces that have similar number of points and similar distributions of normals, but are shaped differently. For example, it helps distinguish an elongated planar surface from a compact planar surface.

Again, the features $\cos \left(\alpha_{i}\right), \cos \left(\phi_{i}\right)$, and $\theta_{i}$ each contain 45 bins, while $\cos \left(\beta_{i}\right)$ has 128 bins, and the new feature $S D C_{i}$ contains 45 bins. The VFH histogram for a cluster is concatenated in the order $\mathcal{H}=[\cos (\alpha), \cos (\phi), \theta, S D C, \cos (\beta)]$ for a total size of 308 bins. The histogram size is consistent between the publication and the PCL implementation. 
To recognize an object in a 3D scene, there must first be a training dataset on which to consult. The training set should contain every distinguishable view of an object. Aldoma et al. consider some specific views of symmetric objects like bowls and bottles as indistinguishable. To match a measured point-cloud to the database, perform the following steps:

1. For every scene histogram, match it to the database by using the distance metric

$$
d(\mathcal{H}, \widetilde{\mathcal{H}})=1-\frac{1+\sum_{i=1}^{308} \min \left(\mathcal{H}_{i}, \widetilde{\mathcal{H}}_{i}\right)}{1+\sum_{i=1}^{308} \max \left(\mathcal{H}_{i}, \tilde{\mathcal{H}}_{i}\right)}
$$

where $\mathcal{H}, \widetilde{\mathcal{H}}$ represent two CVFH histogram descriptors and the subscript $i$ refers to the specific bin.

2. For the best $m_{d}$ matches according to the above distance metric, a camera roll histogram is found. The camera roll histogram is in addition to the 308 bin CVFH histogram, and is used to determine an object's roll about the camera view direction. Transform the cluster's centroid $\overline{\mathbf{p}}$ to coincide with the camera's $z$-axis using the angle-axis representation

$$
\begin{aligned}
\psi & =\arcsin (\|\mathbf{c}\|) \\
\mathbf{e}_{\psi} & =\frac{\mathbf{c}}{\|\mathbf{c}\|}
\end{aligned}
$$

where

$$
\mathbf{c}=\frac{\mathbf{s}_{o}-\overline{\mathbf{p}}}{\left\|\mathbf{s}_{o}-\overline{\mathbf{p}}\right\|} \times \mathbf{s}_{b}
$$

and the camera origin and camera boresight are generally assumed to be $\mathbf{s}_{o}=[0,0,0]^{\top}$ and $\mathbf{s}_{b}=[0,0,1]^{\top}$ respectively. 
3. Now find the angle between the rotated normals and the camera's $z$ axis. The distribution of these angles are placed in the 90 bin camera roll histogram. The number of bins was set based on a trade off between efficiency and accuracy and is consistent with the PCL implementation. For 90 bins, there is a roll accuracy of $4^{\circ}$.

4. To find the rotation angle between two camera roll histograms, apply the Discrete Fourier Transform and multiply the complex coefficients to find the cross power spectrum $R$. The peaks of the spectrum appear at the rotation angles that align the two roll histograms. Only the peaks higher than a threshold $t_{p}$ are used to evaluate rotation. The suggested value is $t_{p}=0.9 * \max (R)$.

5. After aligning the model and scenes views of an object, the initial alignment is refined by ICP.

CVFH is the first of the histogram style descriptors that can be used to determine any arbitrary relative $6 \mathrm{DOF}$ pose of an object due to the inclusion of the camera roll histogram. However, the camera roll histogram results in extra calculations and a larger overall feature histogram.

\subsubsection{Oriented Unique Repeatable Clustered Viewpoint Feature Histogram (OUR-CVFH)}

The Oriented Unique Repeatable Clustered viewpoint Feature His-

togram (OUR-CVFH ) presented by Aldoma et al. [54] is the most recent 
feature histogram descriptor. The main improvement in OUR-CVFH is the creation of a semi-global unique reference frame (SGURF) that - compared to the camera roll histogram — better describes the local coordinate frame for each cluster of points. The SGURF is a repeatable coordinate system that explicitly finds the $6 \mathrm{DOF}$ pose when aligning a model and scene cluster.

To generate the OUR-CVFH , a 3D measured surface is first separated into clusters (see Fig. 2.6 for two examples). Each cluster has an associated centroid and average normal which are used to create the four angular distributions of the surface normals. A SGURF is found for each cluster and placed at its centroid. The distribution of all the points in the surface relative to a cluster's SGURF is then characterized by what we call the interpolated spatial octant component $(I S O C)$. There are five components in total to the OUR-CVFH, of which the concatenation creates a single histogram descriptor. Each cluster will have at least one histogram, with the possibility for a maximum of four histograms - the criteria for which is discussed in the generation of the SGURF.

\subsubsection{Viewpoint Clustering}

Begin with a measured $3 \mathrm{D}$ point-cloud $\mathcal{P}=\left\{\mathbf{p}_{i}, \mathbf{n}_{i}, c_{i}, \epsilon\right\}_{i=1}^{m_{\mathbf{p}}}$, where $\mathbf{p}_{i}$ is a $3 \mathrm{D}$ point, $\mathbf{n}_{i}$ is the associated normal, $c_{i}$ is the associated curvature value, and $\epsilon$ is the spatial resolution (i.e. median distance between points). Note that $\mathbf{p}_{i}$ is a measurement returned by the sensor while $\mathbf{n}_{i}$ and $c_{i}$ are computed quantities. This surface must be separated into clusters $\mathcal{C}_{k}$ where $k=1, \ldots, m_{\mathcal{C}}$. These 
$m_{\mathcal{C}}$ different viewpoint clusters are generated with the following procedure:

1. Remove points from $\mathcal{P}$ that have a curvature value higher than a threshold $t_{c}$. There will be $m_{\mathbf{p}}^{f}$ points remaining in the filtered surface set $\mathcal{P}^{f}=\left\{\mathbf{p}_{i}, \mathbf{n}_{i}\right\}_{i=1}^{m_{\mathbf{p}}^{f}}$.

2. $\mathcal{P}^{f}$ is now separated into smooth and continuous clusters $\mathcal{C}_{k}$. Each cluster is initiated with an arbitrary point in $\mathcal{P}^{f}$ that has not yet been assigned to any cluster. The cluster is smooth if the inner product (dot product) of the normals of two neighboring points is greater than or equal to a threshold $t_{\mathbf{n}}$, and it is continuous if two neighboring points are separated by less than Euclidean distance threshold $t_{d}$. Aldoma et al. [54] set $t_{\mathbf{n}}=$ $\cos \left(8.6^{\circ}\right)$ for personal robotics applications. We find that $t_{\mathbf{n}}=\cos \left(5^{\circ}\right)$ works well for artificial satellites with mostly flat/smooth surfaces, while $t_{\mathbf{n}}=\cos \left(1^{\circ}\right)-\cos \left(3^{\circ}\right)$ degrees works well for natural objects with rough surfaces (e.g. asteroids and comets). To account for measurement noise on the point-cloud, the distance threshold is always set as $t_{d}=2.5 \epsilon$. The constraints are fulfilled by:

$$
\mathcal{C}_{k}^{\prime}=\left\{\mathbf{p}_{i} \in \mathcal{P}^{f}:\left(\left\|\mathbf{p}_{i}-\mathbf{p}_{j}\right\| \leq t_{d}\right) \wedge\left(\mathbf{n}_{j}^{\top} \mathbf{n}_{j} \leq t_{n}\right)\right\}
$$

Each cluster set $\mathcal{C}_{k}^{\prime}=\left\{\left\{\mathbf{p}_{i}, \mathbf{n}_{i}\right\}_{i=1}^{m_{\mathbf{p}, k}^{\prime}}, \overline{\mathbf{p}}_{k}, \overline{\mathbf{n}}_{k}\right\}_{k=1}^{m_{\mathcal{e}}^{\prime}}$ has an associated centroid 
$\overline{\mathbf{p}}$ and average normal $\overline{\mathbf{n}}$ defined as

$$
\begin{aligned}
& \overline{\mathbf{p}}=\frac{1}{m_{\mathbf{p}, k}^{\prime}} \sum_{i}^{m_{\mathbf{p}, k}^{\prime}} \mathbf{p}_{i} \\
& \overline{\mathbf{n}}=\frac{\frac{1}{m_{\mathbf{p}, k}^{\prime}} \sum_{i}^{m_{\mathbf{p}, k}^{\prime}} \mathbf{n}_{i}}{\left\|\frac{1}{m_{\mathbf{p}, k}^{\prime}} \sum_{i}^{m} \mathbf{n}_{i}\right\|}
\end{aligned}
$$

3. Each cluster is filtered once more by the angle between the normal of each point and the cluster's average normal by the angle threshold $t_{\overline{\mathbf{n}}}$ to make the cluster

$$
\mathcal{C}_{k}=\left\{\mathbf{p}_{i} \in \mathcal{C}_{k}^{\prime}:\left(\overline{\mathbf{n}}_{k}^{\top} \mathbf{n}_{i} \leq t_{\overline{\mathbf{n}}}\right)\right\}
$$

PCL Aldoma et al. set $t_{\bar{n}}=\cos \left(0.89^{\circ}\right)$, where we set it as $t_{\overline{\mathbf{n}}}=t_{\mathbf{n}} / 10$, where $t_{\mathbf{n}}$ was set in the previous step. The difference is that Aldoma et al. [54] work with a well regulated scene, where we are working with a dynamically changing object in space. Only clusters that still contain more than a minimum number of points are kept. The cluster set is $\mathcal{C}_{k}=\left\{\left\{\mathbf{p}_{i}, \mathbf{n}_{i}\right\}_{i=1}^{m_{\mathbf{p}, k}}, \overline{\mathbf{p}}_{k}, \overline{\mathbf{n}}_{k}\right\}_{k=1}^{m_{\mathrm{e}}}$. The centroid $\overline{\mathbf{p}}_{k}$ and surface normal $\overline{\mathbf{n}}_{k}$ of set $\mathcal{C}_{k}$ are the same as set $\mathcal{C}_{k}^{\prime}$ and are not recalculated.

\subsubsection{Semi-Global Unique Reference Frame (SGURF)}

Now that the surface $\mathcal{P}^{f}$ has been separated into smooth, continuous clusters $\mathcal{C}_{k}$, a SGURF is calculated for each. The purpose of the SGURF is to increase the spatial descriptiveness of the histogram and is used to directly find the $6 \mathrm{DOF}$ pose when aligning reference frames. 
The origin of $\mathrm{SGURF}_{k}$ is fixed at the centroid $\overline{\mathbf{p}}_{k}$ of the cluster $\mathcal{C}_{k}$. The orientation of $\mathrm{SGURF}_{k}$ depends on the spatial distribution of the points on the entire point-cloud $\mathcal{P}$. If the orientation is ambiguous, multiple SGURFs will be calculated for a given cluster $\mathcal{C}_{k}$, resulting in multiple OUR-CVFH histograms for a given cluster. Define a cluster's SGURF as

1. For each cluster $\mathcal{C}_{k}$, compute the eigen-decomposition of the scatter ma$\operatorname{trix}[54,88]$

$$
\mathbf{M}=\frac{1}{\sum_{i}^{m_{\mathbf{p}, k}}\left(d_{\max }-d_{i}\right)} \sum_{i}^{m_{\mathbf{p}, k}}\left(R-d_{i}\right)\left(\mathbf{p}_{i}-\overline{\mathbf{p}}_{k}\right)\left(\mathbf{p}_{i}-\overline{\mathbf{p}}_{k}\right)^{\top}
$$

where $d_{i}=\left\|\mathbf{p}_{i}-\overline{\mathbf{p}}_{k}\right\|$ and $d_{\max }=\max _{\mathbf{p}_{i} \in \mathfrak{C}_{k}}\left\|\mathbf{p}_{i}-\overline{\mathbf{p}}_{k}\right\|$.

2. The eigenvector corresponding to the smallest eigenvalue is chosen as the $z$-axis of $\mathrm{SGURF}_{k}$. The $z$-axis will point in the same direction as the cluster average normal $\overline{\mathbf{n}}_{k}$. Therefore, take the direction of $z$-axis yielding a positive dot product with $\overline{\mathbf{n}}_{k}$

3. The sign of the other axes must now be disambiguated. Temporarily call the remaining eigenvectors as $\left(\mathbf{e}_{1}^{+}, \mathbf{e}_{2}^{+}\right)$and their opposites as $\left(\mathbf{e}_{1}^{-}, \mathbf{e}_{2}^{-}\right)$. In an attempt to uniquely and repeatable identify the $x$-axis from the $y$-axis, we use the whole point-cloud $\mathcal{P}$ to evaluate the point density of the hemispheres defined by each eigenvector. The point densities are found as

$$
\Psi_{\mathrm{e}_{1}}^{ \pm}=\sum_{i=1}^{m_{\mathbf{p}}} \begin{cases}\left|\psi_{i}^{ \pm}\right| \psi_{i}^{ \pm} & \text {if } \psi_{i}^{ \pm} \geq 0 \\ 0 & \text { otherwise }\end{cases}
$$


where

$$
\psi_{i}^{ \pm}=\left(\mathbf{p}_{i}-\overline{\mathbf{p}}_{k}\right)^{\top} \mathrm{e}_{1}^{ \pm}
$$

and similarly for $\mathbf{e}_{2}$. Then the sign of $\mathbf{e}_{1}$ is disambiguated as

$$
\mathbf{e}_{1}= \begin{cases}\mathbf{e}_{1}^{+} & \text {if }\left|\Psi_{\mathbf{e}_{1}}^{+}\right| \geq\left|\Psi_{\mathbf{e}_{1}}^{-}\right| \\ \mathbf{e}_{1}^{-} & \text {otherwise }\end{cases}
$$

and the sign of $\mathbf{e}_{2}$ is disambiguated analogously.

4. To decide which vector e corresponds to each axis, calculate the disambiguation factor $f_{1}, f_{2}$ as

$$
\mathbf{f}_{j}=\frac{\min \left(\left|\Psi_{\mathbf{e}_{j}}^{+}\right|,\left|\Psi_{\mathbf{e}_{j}}^{-}\right|\right)}{\max \left(\left|\Psi_{\mathbf{e}_{j}}^{+}\right|,\left|\Psi_{\mathbf{e}_{j}}^{-}\right|\right)}, \quad j=1,2
$$

The disambiguation factor $\mathrm{f}_{j} \in[0-1]$, where $\mathrm{f}_{j}=0$ is complete disambiguation and $\mathbf{f}_{j}=1$ is complete ambiguity.

5. The eigenvector with the lower disambiguation factor is chosen as the $x$-axis of $\mathrm{SGURF}_{k}$.

6. The $y$-axis of $\mathrm{SGURF}_{k}$ is found as $y=z \times x$, creating a right handed coordinate system. The original publication [54] defined a left handed coordinate system, but the convention in the aerospace community is to be right handed.

7. In some situations - for example if the cluster $\mathcal{C}_{k}$ is nearly circular and is centrally located on the point-cloud $\mathcal{P}$ - the axis disambiguation is 
not robust and the disambiguation factors are similar. Calculate the factor ratio as

$$
r_{f}=\frac{\min \left(f_{1}, f_{2}\right)}{\max \left(f_{1}, f_{2}\right)}
$$

If the ratio is greater than a threshold $t_{\mathrm{r}_{\mathrm{f}}}$, the we must create two reference frames - one frame with $\mathbf{e}_{1}$ as the $x$-axis and the other using $\mathbf{e}_{2}$. Along with PCL, we set this value as $t_{\mathrm{r}_{\mathrm{f}}}=0.8$. If both disambiguation factors are similar and greater than a threshold $t_{\mathrm{f}}$, then we create four reference frames - one for each eigenvector for each sign as the $x$-axis. Once again, we and PCL both use $t_{\mathrm{f}}=0.925$ for this comparison value.

The number of histograms per cluster is dependent on the number of SGURFs created for that cluster. Each cluster may have up to four SGURFs, which would result in up to four descriptors. Only the ISOC histogram changes because of the SGURF. The angular distribution components remain constant for a given cluster. A cluster with its SGURF on the surface of Itokawa is shown in Fig. 2.1 and its accompanying histogram is shown in Fig. 2.4.

Before using the SGURF for cluster description or pose estimation, we must find the transformation from the camera frame $C$ to the SGURF frame denoted as $F$. The transformation is found with the following procedure:

1. Calculate the viewpoint vector $\mathbf{v}$ as

$$
\mathbf{v}=\frac{\mathbf{s}_{o}-\overline{\mathbf{p}}_{k}}{\left\|\mathbf{s}_{o}-\overline{\mathbf{p}}_{k}\right\|}
$$

where the camera origin usually assumed to be $\mathbf{s}_{o}=[0,0,0]^{\top}$. 
2. Take the cross product of the viewpoint vector with the camera boresight as

$$
\mathbf{c}=\mathbf{v} \times \mathbf{s}_{b}
$$

where the boresight is generally assumed to be $\mathbf{s}_{b}=[0,0,1]^{\top}$.

3. Convert $\mathbf{c}$ into Rodrigues parameters in terms of the Euler axis and angle as

$$
\begin{aligned}
\psi & =\arcsin (\|\mathbf{c}\|) \\
\mathbf{e}_{\psi} & =\frac{\mathbf{c}}{\|\mathbf{c}\|}
\end{aligned}
$$

where $\psi$ is the rotation angle, and $\mathbf{e}_{\psi}$ is the axis of rotation.

4. Combine $\psi$ and $\mathbf{e}_{\psi}$ using Rodriques' rotation formula to make a rotation matrix $\mathbf{R}$ from the sensor frame $S$ to an intermediate frame $I$ that lies along the camera boresight

$$
\mathbf{R}_{I}^{C}=\cos (\psi) \mathbf{I}_{3 \times 3}+[1-\cos (\psi)] \mathbf{e}_{\psi} \mathbf{e}_{\psi}^{\top}-\sin (\psi)\left[\mathbf{e}_{\psi} \times\right]
$$

where $[\cdot \times]$ is the skew-symmetric cross product matrix. Also recall that the $\mathrm{SGURF}_{k j}$ is the local coordinate system fixed at $\overline{\mathbf{p}}_{k}$. It may also be viewed as a frame transformation $\mathbf{R}_{I}^{F}$ from the SGURF frame $F$ to the intermediate frame $I$. To rotate and translate a point in the sensor frame to the SGURF frame, use

$$
\begin{aligned}
\mathbf{R}_{F}^{S} & =\mathbf{R}_{F}^{I} \mathbf{R}_{I}^{C} \\
\mathbf{t}_{F} & =-\mathbf{R}_{F}^{I} \mathbf{R}_{I}^{S} \overline{\mathbf{p}}_{S}
\end{aligned}
$$


where $\mathbf{R}_{F}^{S}$ is the rotation from the sensor frame $S$ to the SGURF frame $F$. Also, $\mathbf{t}_{F}$ is the location of the SGURF frame origin with respect to the sensor frame origin as expressed in the SGURF frame.

5. Both the rotation and translation may be performed in a single operation with the use of a homogeneous transformation (sometimes just referred to as transformation). Thus, define $\mathbf{T}_{F}^{S}$ to be the transformation from the sensor frame $S$ into the local SGURF frame $F$

$$
\mathbf{T}_{F}^{S}=\left[\begin{array}{cc}
\mathbf{R}_{F}^{S} & \mathbf{t}_{F} \\
\mathbf{0}_{1 \times 3} & 1
\end{array}\right]
$$

This transformation will be used to estimate pose and to describe ISOC component of the histogram.

\subsubsection{Histogram Generation}

Each cluster may now be described by the five components of the histogram. Each cluster will have at least one histogram, with a possible maximum of four depending on the SGURF generation rules. The five components are found as follows.

1. First define a Darboux coordinate frame $D=(\mathbf{u}, \mathbf{v}, \mathbf{w})$ for every point $\mathbf{p}_{i} \in \mathcal{C}_{k}$ defined as

$$
\begin{aligned}
\mathbf{u}_{i} & =\overline{\mathbf{n}}_{k} \\
\mathbf{v}_{i} & =\frac{\left(\mathbf{p}_{i}-\overline{\mathbf{p}}_{k}\right) \times \mathbf{u}_{i}}{\left.\| \mathbf{p}_{i}-\overline{\mathbf{p}}_{k}\right) \times \mathbf{u}_{i} \|} \\
\mathbf{w}_{i} & =\mathbf{u}_{i} \times \mathbf{v}_{i}
\end{aligned}
$$




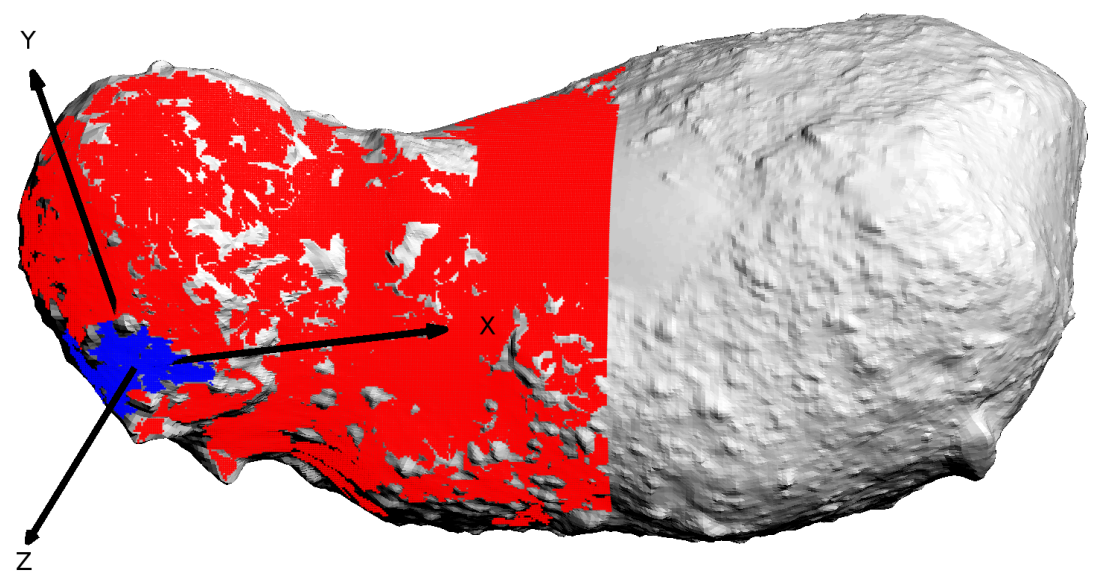

Figure 2.1: A cluster in blue with the rest of the measured point-cloud in red. The SGURF is centered on the cluster's centroid. To aid in disambiguation, the $x$-axis of the SGURF directs towards the rest of the point-cloud and along the body of Itokawa.

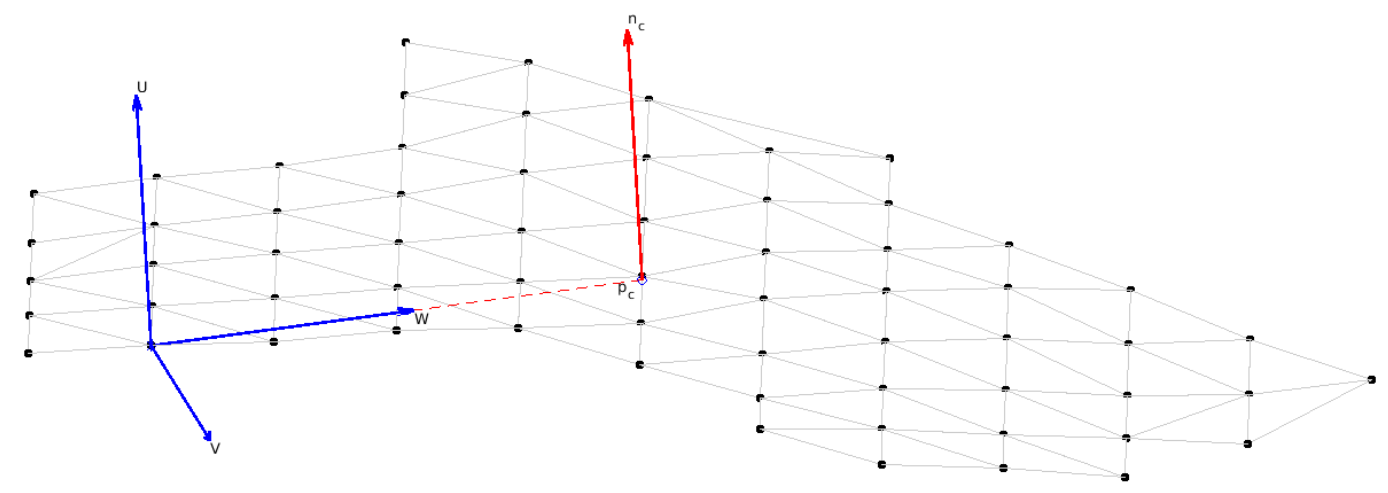

Figure 2.2: A Darboux frame $\mathrm{D}$ is a local right handed coordinate system for a point on a patch. Notice that $\mathbf{u}$ is the parallel to the average normal $\mathbf{n}_{c}$. When looking at the patch from the camera viewpoint, $\mathbf{v}$ directs counter-clockwise for all points. Also notice that $\mathbf{w}$ is nearly parallel with the line connecting the point and the cluster centroid, and is directed towards the centroid $\overline{\mathbf{p}}_{c}$ 
An example of a Darboux Frame for a smooth continuous cluster is shown in Fig. 2.2.

2. Calculate the first, second, third, and fifth components of the histogram. These are the angular distributions of the normals for the cluster calculated as

$$
\begin{aligned}
\cos \left(\alpha_{i}\right) & =\mathbf{v}_{i}^{\top} \mathbf{n}_{i} \\
\cos \left(\phi_{i}\right) & =\mathbf{u}_{i}^{\top}\left(\frac{\mathbf{p}_{i}-\overline{\mathbf{p}}_{k}}{\left\|\mathbf{p}_{i}-\overline{\mathbf{p}}_{k}\right\|}\right) \\
\theta_{i} & =\arctan \left(\frac{\mathbf{w}_{i}^{\top} \mathbf{n}_{i}}{\mathbf{u}_{i}^{\top} \mathbf{n}_{i}}\right) \\
\cos \left(\beta_{i}\right) & =\mathbf{n}_{i}^{\top} \mathbf{v}
\end{aligned}
$$

where $\cos \left(\alpha_{i}\right), \cos \left(\phi_{i}\right)$, and $\theta_{i}$ are encoded with 45 bins each while $\cos \left(\beta_{i}\right)$ uses 64 bins, and $\mathbf{v}$ of Eq. 2.83 is from Eq. 2.69. To gain a physical intuition about these features, examine Fig. 2.3.

3. The fourth component is a spatial description of $\mathcal{P}$ by means of SGURF called the ISOC. Use the homogeneous transformation from Eq. 2.76 to transform the point-cloud $\mathcal{P}$ from the camera frame $C$ to the local SGURF frame $F$. Now the points in $\mathcal{P}$ are naturally divided into octants described by the signed axes $\left(x^{ \pm}, y^{ \pm}, z^{ \pm}\right)$.

4. In order to account for variations on SGURF due to measurement noise or partial occlusions, Aldoma et al. [54] interpolate the distribution of points in the octants. Begin by placing a 1D Gaussian function over each of the $x-, y$-, and $z$-axes with standard deviations $\sigma_{i}=0.01$. Because this 


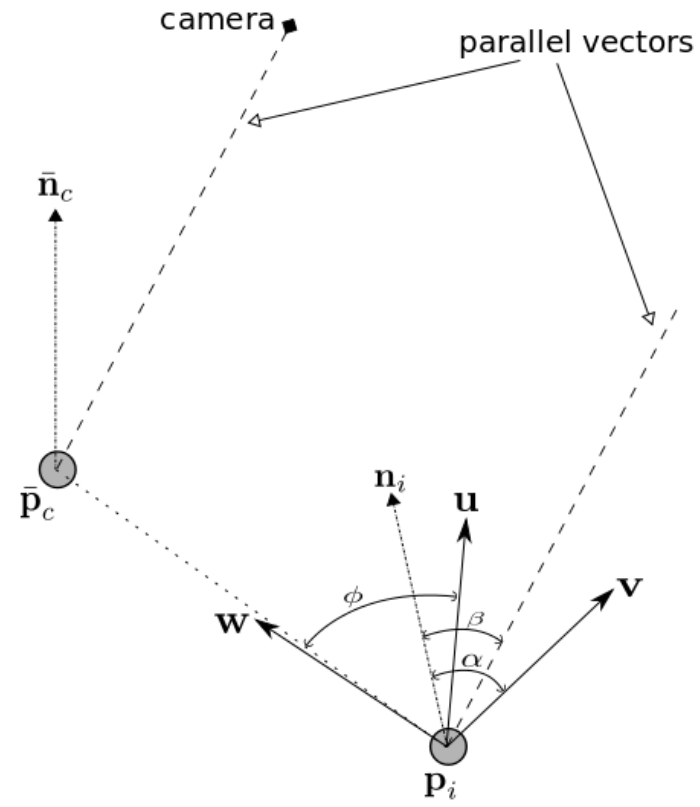

Figure 2.3: To better understand the meaning of the feature angles, consider the local Darboux Frame at an arbitrary point $\mathbf{p}_{i}$ defined as $\mathrm{D}=(\mathbf{u}, \mathbf{v}, \mathbf{w})$. The angle between $\mathbf{v}$ and the surface normal $\mathbf{n}_{i}$ at $\mathbf{p}_{i}$ is $\alpha$. The angle between $\mathbf{u}$ and the line connecting the cluster centroid $\overline{\mathbf{p}}_{c}$ to $\mathbf{p}_{i}$ is $\phi$. The angle between $\mathbf{n}_{i}$ and the line parallel to the line connecting the camera to $\overline{\mathbf{p}}_{c}$ is $\beta$. The meaning of $\theta$ is difficult to explain graphically so is not shown. 
interpolation scheme describes the likelihood that a particular point lies in a particular octant, this Gaussian weighting describes the likelihood that SGURF axes uncertainty could cause a point to appear in a different octant. Assign each point $\mathbf{p}_{i} \in \mathcal{C}_{k}$ three position weights determined by their $x-, y$-, and $z$-coordinates

$$
w_{i, \alpha}=1-\mathrm{G}\left(\sigma, \mathbf{p}_{i, \alpha}\right), \quad \alpha=x, y, z
$$

where $\alpha$ is cycling through the axes and and $i=1, \ldots, m_{\mathfrak{C}_{k}}$. There is high likelihood that the point lies in a given octant as $w_{i, \alpha} \rightarrow 1$, whereas the point lies near an octant boundary when $w_{i, \alpha} \rightarrow 0$.

5. ISOC weights are computed through multiplication of the position weights and summation over the octants

$$
\mathbf{W}_{i, \eta}=\sum_{\eta} \prod_{\alpha} 0.5\left(1+\operatorname{sgn}\left(\mathbf{p}_{i, \alpha}\right) \delta(\alpha, \eta) w_{i, \alpha}\right)
$$

where $\mathbf{W}_{i, \eta}$ is of size $m_{\mathfrak{C}_{k}} \times 8$, summation is over the octant $\eta=1, \ldots, 8$, and the unit impulse $\delta(\alpha, \eta)$ takes the sign

$\delta(\eta, \alpha)=$\begin{tabular}{|c|c|c|c|c|c|c|c|c|c|}
\hline & \multicolumn{10}{|c|}{ Octant $\eta$} \\
\hline & & 1 & 2 & 3 & 4 & 5 & 6 & 7 & 8 \\
\hline & $x$ & - & - & - & - & + & + & + & + \\
& $y$ & - & - & + & + & - & - & + & + \\
& $y$ & + & - & + & - & + & - & + & - \\
\hline
\end{tabular}

depending on both the octant and point element.

6. The histogram values for each octant are found by a summation of the position weights of all the points falling into a specific bin. Each octant is 
separated into 13 bins dependent on a point's normalized distance from the origin. The bin in which a weight is accumulated is found as

$$
b_{i}=\left\lfloor 12 \frac{\left\|\mathbf{p}_{i}-\overline{\mathbf{p}}_{k}\right\|}{\max _{\mathbf{p}_{i} \in \mathcal{C}_{k}}\left\|\mathbf{p}_{i}-\overline{\mathbf{p}}_{k}\right\|}\right\rfloor
$$

where $\lfloor\cdot\rfloor$ is the floor operator, and resulting in a total ISOC size of 104 bins. Eq. 2.87 results in bin values of $b_{i} \in[0,12]$ which is 13 elements long. ISOC is found by vectorizing $\mathbf{W}_{i, \eta}$.

The complete OUR-CVFH histogram descriptor contains 303 bins combined in the order $\mathcal{H}=[\cos (\alpha), \cos (\phi), \theta, I S O C, \cos (\beta)]$. Recall that if a cluster has multiple SGURFs, then the ISOC description will be different for each $\mathrm{SGURF}_{k j}$, which results in multiple histograms. An example of a histogram for a feature is shown in Fig. 2.4.

\subsubsection{Database Training}

Each of the histogram style point-cloud descriptors must go through a training session to create a database of features. These models are used in the recognition framework to identify an object and to provide a pose estimate. Training is performed by using either (1) using an actual 3D sensor to take range images of a real object at various ranges and attitudes or (2) using a 3D sensor simulator and a 3D mesh model.

OUR-CVFH virtually renders a 3D model and simulates LIDAR scans from various viewing angles, but only at a constant range. Place the 3D model at the origin of a polyhedron with equally spaced vertices. 


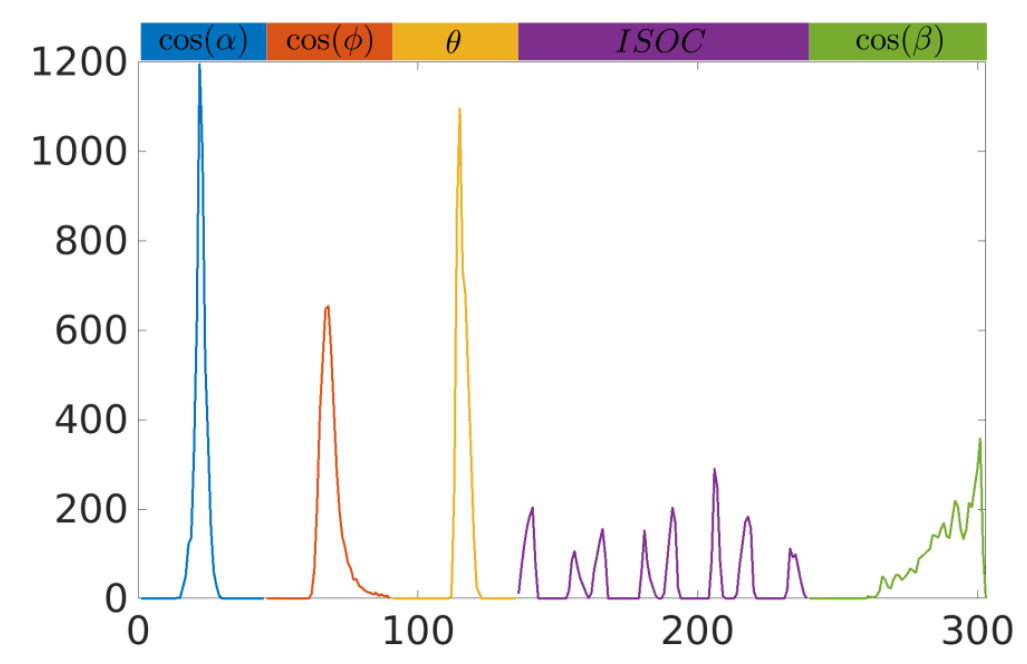

Figure 2.4: An example of the OUR-CVFH histogram. The features are concatenated and color labeled. The size of the patch is implicitly contained in the histogram as it counts the total number of points in the patch.

Aldoma et al. [54] assume that the robot maintains a constant distance from all objects so that the scene resolution is also nearly constant at $3 \mathrm{~mm}$. The assumption of a common resolution is often unsatisfied in practice. During recognition, they first using moving least square (MLS) to up-sample the point-cloud. The resulting point-cloud is down-sampled to the desired resolution of $3 \mathrm{~mm}$. The simulated 3D sensor is then placed at each of the vertices of the polyhedron and viewpoint dependent point-clouds are taken. The resulting training set must contain the homogeneous transformations $\mathbf{T}_{F}^{S}$, and all the points in each point-cloud $\mathcal{P}$ along with each OUR-CVFH descriptor $\mathcal{H}$. Together, these components create the set $\mathcal{M}=\left\{\mathbf{T}_{F}^{S}, \mathcal{H}, \mathcal{P}\right\}$. 


\subsubsection{Recognition Framework}

The recognition stage of OUR-CVFH provides a 6DOF relative pose estimate. Given a measured surface from a 3D sensor (e.g. time-of-flight camera, LIDAR), follow the steps in Sec. 2.2.5.4 to separate the surface into clusters and generate OUR-CVFH descriptors. Call these descriptors of the measured POINT-CLO as the set $\mathcal{S}=\left\{\widetilde{\mathbf{T}}_{F}^{S}, \widetilde{\mathcal{H}}, \widetilde{\mathcal{P}}\right\}$, where $\widetilde{\mathcal{P}}$ is the entire measured pointcloud.

Recall the training set created on the computer model of the same object as $\mathcal{M}=\left\{\mathbf{T}_{F}^{S}, \mathcal{H}, \mathcal{P}\right\}$ which contains all descriptors for all the training views. The matching process is as follows:

1. For every descriptor $\widetilde{\mathcal{D}} \in \mathcal{S}$, match it to every descriptor $\mathcal{H} \in \mathcal{M}$ using the distance metric

$$
\mathrm{d}(\widetilde{\mathcal{H}}, \mathcal{H})=1-\frac{1+\sum_{i=1}^{303} \min \left(\widetilde{\mathcal{H}}_{i}, \mathcal{H}_{i}\right)}{1+\sum_{i=1}^{303} \max \left(\widetilde{\mathcal{H}}_{i}, \mathcal{H}_{i}\right)}
$$

A graphical interpretation of histogram matching is shown in Fig. 2.5. Find the $m_{d}$ number of descriptor pairs that produce the smallest distance metric. We found $m_{d}=10$ to work well.

2. For the best $m_{d}$ matches with the smallest distance metric, find the relative $6 \mathrm{DOF}$ pose of the initial alignment for ICP as

$$
\delta \widetilde{\mathbf{T}}_{S}^{-}=\widetilde{\mathbf{T}}_{S}^{F} \mathbf{T}_{F}^{S}
$$




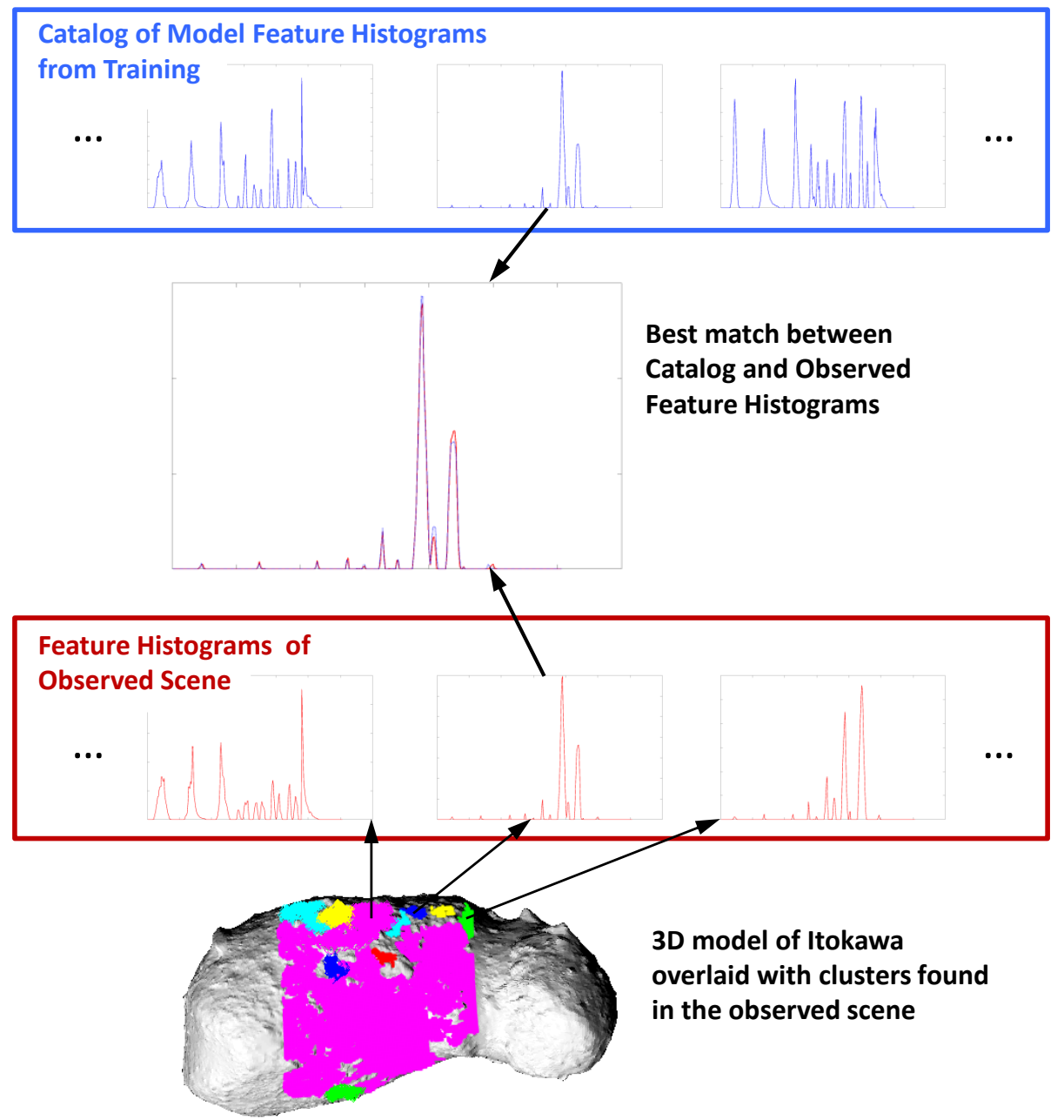

Figure 2.5: Illustration of the best match between a set of feature histograms found in a measured 3D point-cloud (red box) and a catalog of known feature histograms (blue box). Every observed scene feature histogram is matched to every catalog feature histogram. The best match (as determined by Sec. 2.2.5.5) is the pair of overlaid histograms in the center. For histograms from the observed scene, their corresponding cluster is shown in the image of Itokawa. Note here that the largest cluster (the magenta cluster) was not used to estimate pose. This process is influenced by the parameter $\lambda$ in Eq. 2.91. 
where $\delta \widetilde{\mathbf{T}}_{S}^{-}$is the difference between the training and sensed SGURF poses and the (-) superscript denotes before ICP improvement. Use ICP to update the relative pose estimate to yield $\delta \widetilde{\mathbf{T}}_{S}^{+}$.

3. The 6 DOF relative pose from the object body $B_{\S}$ in the training set to the object body $B_{\mathcal{M}}$ is

$$
\mathbf{T}_{B_{\mathcal{M}}}^{B_{\mathcal{S}}}=\delta \widetilde{\mathbf{T}}_{S}^{+}
$$

4. To improve the recognition stage and attempt to address the topic of scale, Aldoma et al. [54] add an additional step to the histogram matching. This step gives preference to to the number of inliers and outliers of the point-clouds. With the point-clouds in the same reference frame, a point in the model cloud is considered an inlier if the distance to a point in the scene cloud is within a threshold distance $t_{d}$, otherwise it is an outlier. This threshold is set as $t_{d}=2 \epsilon$ where $\epsilon$ is still the spatial resolution of the scene point-cloud. Then a cost metric is calculated as

$$
J=m_{\text {in }}-\lambda m_{\text {out }}
$$

where $m_{\text {in }}$ is the number of inliers, $m_{\text {out }}$ is the number of outliers, and $\lambda$ is used to weight the outlier count. Neither Aldoma et al. [54] nor PCL provide a range for this weight, so we set it as $\lambda=1$. Of the $m_{d}$ best matches from Step 1, the best candidate model cluster (and pose estimate) is the one that maximizes the metric $J$. 

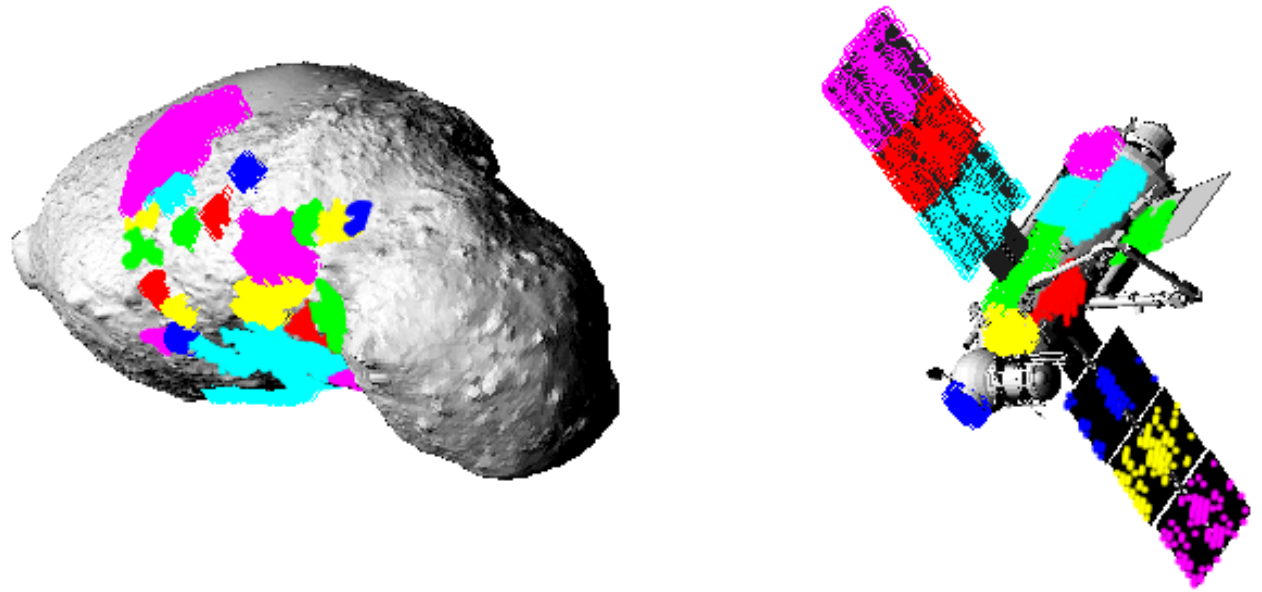

Figure 2.6: Example OUR-CVFH clusters on the asteroid Itokawa (left) and on a module from the International Space Station (right). Each cluster is represented by a different color. If two clusters have the same color but are clearly separated, then they indicate different clusters. 


\section{Chapter 3}

\section{Signal Diffusion on 3D Meshes and Point-Clouds}

Isotropic diffusion is governed by the heat equation and implemented with discrete approximations $[89,90]$. The in-surface isotropic diffusion equation

$$
\frac{\partial u}{\partial t}=\alpha \nabla_{\mathcal{S}}^{2} u
$$

diffuses a function $u$ along the 3D surface $\mathcal{S}$, where $t$ is the diffusion time (related to the scale-parameter), $\alpha$ is the constant of diffusion, and $\nabla_{\S}^{2}$ is the continuous Laplace-Beltrami operator (LBO) which is a generalization of the Laplace operator to surfaces. The characteristic solution (i.e. the impulse response) to Eq. 3.1 is sometimes referred to as the heat kernel [91], which is the well-known Gaussian used for 1D signal analysis [89, 92] and 2D image scale-space $[52,49]$. For surfaces embedded in $\mathbb{R}^{3}$, the solution to the diffusion equation is less straightforward.

Implementation of Eq. 3.1 on a mesh may be accomplished through either of two discretization methods. The first method requires the discretization in space of $\nabla_{\mathcal{S}}^{2}$ using a discrete LBO. Many numerical methods are available to solve the resulting semi-discrete equation, some of which also depend on 
Table 3.1: Combinations of the two solution methods to the diffusion equation and the two surface representations combine to make four implementation techniques.

\begin{tabular}{lcc}
\hline \hline & $\begin{array}{c}\text { Gaussian } \\
\text { Convolution }\end{array}$ & $\begin{array}{l}\text { Analytic } \\
\text { Discretization }\end{array}$ \\
\hline Explicit Surface & GE & DE \\
Implicit Surface & GI & DI \\
\hline \hline
\end{tabular}

a linearization in time. The second method utilizes the well-known characteristic solution to the diffusion equation, the Gaussian. In this method, the distance calculation between points is of interest with the two most common types being Euclidean and geodesic distance.

In addition to the two methods of discretization, there are two types of surface representation. The first type uses the explicit surface as given (e.g. in formats such as .ply or .off) and in the native dimensional embedding. The second type embeds the explicit surface in a Cartesian grid for an implicit representation. The combination of solution method and surface representation leads to four methods of implementing the diffusion equation on 3D surfaces. These methods and their acronyms used this work are shown in Table 3.1. The implementations and limitations of these techniques are discussed.

\subsection{Surface, Mesh, and Grid Definitions}

This section offers an introduction to notation and terminology for surfaces, meshes, and embedding grids. Surfaces are discretely represented by 


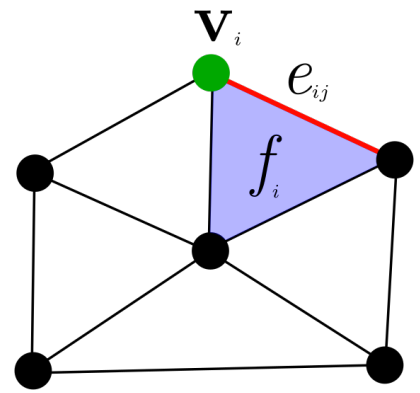

Figure 3.1: Triangular mesh definitions. A vertex $\mathbf{v}_{i}$ is connected by an edge $e_{i j}$ on the face $f_{i}$.

an explicit mesh for diffusion on explicit surfaces, with a good review given by [93]. It is utilized in Section 3.4 and Section 3.5. The explicit mesh is embedded into a Cartesian grid for diffusion on implicit surfaces. The embedding grid is utilized in Section 3.2 and with minor adjustments in Section 3.3.

\subsubsection{Surfaces and Meshes}

Suppose an explicit surface $\mathcal{S}$ is discretely represented by the explicit mesh $\mathcal{M}$. The abstract simplicial complex $\mathcal{M}=\{\mathcal{V}, \mathcal{E}, \mathcal{F}, \mathcal{U}\}$ contains the vertex subset $\mathcal{V}=\left\{\mathbf{v}_{i}\right\}_{i=1}^{m_{\mathrm{v}}}$, the subset of edges $\mathcal{E}=\left\{e_{i j}\right\}$ between vertices, the subset of faces $\mathcal{F}=\left\{f_{i}\right\}_{i=1}^{m_{f}}$, and the signal subset $\mathcal{U}=\left\{u_{i}\right\}_{i=1}^{m_{\mathrm{v}}}$. The signal $u_{i}$ exists at the $i$-th vertex of the mesh $\mathbf{v}_{i}$ which is connected to $\mathbf{v}_{j}$ via edge $e_{i j}$ and exists between two oriented faces. A compact representation for the homogeneity of the mesh is given by the local and global average edge lengths

$$
\bar{e}=\frac{\sum e_{i j}}{|\mathcal{E}|} \quad \bar{e}_{i}=\frac{\sum_{i j \in \mathcal{N}\left(\mathbf{v}_{i}\right)} e_{i j}}{\left|\mathcal{N}\left(\mathbf{v}_{i}\right)\right|}
$$




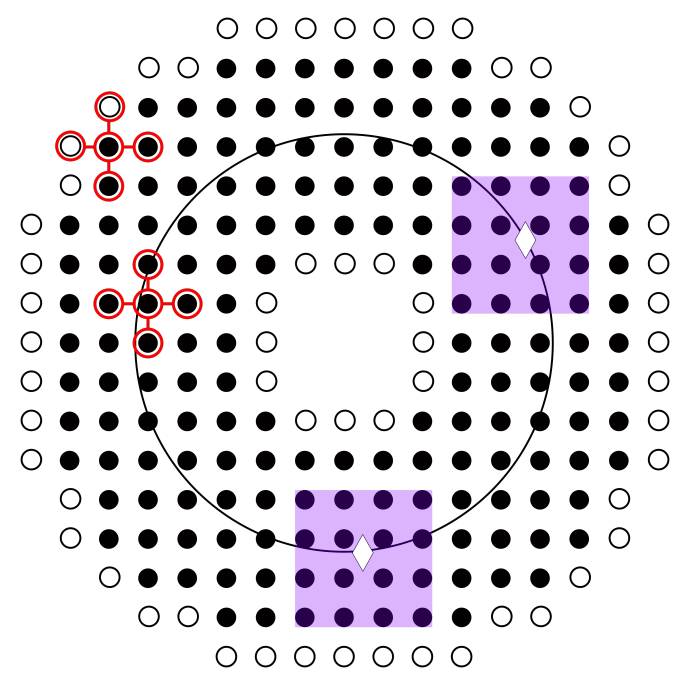

Figure 3.2: Embedding grid definitions. A mesh $\mathcal{M}$ is a circle, embedded in a grid of $\mathcal{B}$ (indicated by $\bullet$ ), and the ghost points $\mathcal{G}$ (indicated by $\circ$ ). For a point on $\mathcal{M}$ (indicated with $\diamond$ ), the $4 \times 4$ interpolation stencil is shown in purple. The five point Laplace stencil is shown in red. This figure is reproduced from [1].

respectively, where $\mathcal{N}\left(\mathbf{v}_{i}\right)$ is the first-ring neighborhood of a vertex and $|\cdot|$ returns the cardinality of the set. While this work uses triangulated surfaces, any mesh with flat convex polygon faces may construct a surface. A portion of a triangular surface is shown in Fig. 3.1 to highlight components of the mesh.

\subsubsection{Embedding Grids}

Now suppose that the mesh $\mathcal{M}$ is embedded into an $\mathbb{R}^{3}$ Cartesian grid such that every point $\mathbf{x} \in \mathbb{R}^{3}$ has a corresponding (possibly non-unique) closest point $\operatorname{cp}(\mathbf{x}) \in \mathcal{M}$ that is closest in Euclidean distance. Define the embedding grid as the mesh complement $\overline{\mathcal{M}}=\left\{\mathcal{B}, \mathcal{G}, \mathcal{U}_{\mathcal{B}}, \mathcal{U}_{\mathcal{G}}\right\}$. The abstract simplicial 
complex consists of the points $\mathcal{B}=\left\{\mathbf{x}_{i}\right\}_{i=1}^{m_{\mathcal{B}}}$ within the interpolation stencil, the additional set of ghost points $\mathcal{G}=\left\{\mathbf{x}_{i}\right\}_{i=1}^{m_{\mathcal{G}}}$ outside of the stencil, but used to ensure that $\mathcal{B}$ is accurately computed, the signal $\mathcal{U}_{\mathcal{B}}=\left\{u\left(\operatorname{cp}\left(\mathbf{x}_{i}\right)\right)\right\}_{i=1}^{m_{\mathcal{B}}}$ on the points in $\mathcal{B}$, and the signal $\mathcal{U}_{\mathcal{G}}=\left\{u\left(\operatorname{cp}\left(\mathbf{x}_{i}\right)\right)\right\}_{i=1}^{m_{\mathcal{G}}}$ on the points in $\mathcal{G}$. A sample 2D surface and grid are shown in Fig. 3.2 to highlight components of the embedding grid.

The signal existing on the explicit surface is represented by $\mathcal{U}$ and is extended constant normal onto $\mathbf{x} \in \mathbb{R}^{3}$ such that $u(\mathbf{x})=u(\operatorname{cp}(\mathbf{x}))$. Diffusion only occurs along - not orthogonal to - the surface with this method of data extension. At each time step, a combination of diffusion on the points $\mathbf{x}$ and interpolation of $u(\operatorname{cp}(\mathbf{x}))$ occurs, followed by an implied reassigning of $u(\mathbf{x})$.

\subsection{Discretized Diffusion on Implicit Surfaces (DI)}

A surface may be represented implicitly by embedding the mesh in a Cartesian grid. Each point in the grid is used to define the surface based on its distance. This description of the 3D surface is agnostic to mesh construction (e.g. edge length, polygonal facets). With this representation, well-studied numerical techniques of the gradient, divergence, and Laplacian with Euclidean operations are utilized on the embedding grid. Then the solution is interpolated back onto the explicit surface. Two popular methods of implicit surface description are (1) level sets using the signed distance field (SDF) and (2) the closest point representation (CPR).

Level set methods with SDF define the original surface as the zero level 
set. For each Cartesian point, the SDF defines a distance to the zero level set along with a corresponding sign determining if the point is interior or exterior to the surface. The diffusion equation is solved using forward-in-time and backward-in-space discretization [94], or forward-in-time approximation followed by an iterative solver [95]. Disadvantages of level set method with SDFs are that (1) they are defined on all of space and time, and thus computationally expensive to solve, (2) there lacks a definition of what occurs if a point is equidistant from two locations on the zero level set, and (3) since the distance is signed, they cannot be used to describe open surfaces, or surfaces lacking orientation (e.g. Möbius strip).

The CPR is a paradigm for implicitly representing surfaces to solve a variety of partial differential equations (PDEs). It is distinguished from the level set method with SDF because it (1) solves PDEs for only one time step before reinitializing, (2) only operates on a narrow band around the surface, and (3) since the distance is unsigned, works well with open, closed, and non-orientable surfaces. CPR was developed over a series of publications where the authors use forward-in-time discretization with the Closest Point Method (CPM) [96] and backward-in-time discretization with the Implicit Closest Point Method (ICPM) [1]. $\mathrm{CPR}^{1}$ was theoretically proven in [97]. We use the $\mathrm{ICPM}^{2}$ to describe an implicit mesh and solve Eq. 3.1 using a first-order backward-in-time Euler scheme. This approach was presented in [58] for scale-space construction

\footnotetext{
${ }^{1}$ We use the acronym CPR to encompass both CPM and ICPM.

${ }^{2}$ Code for the ICPM may be found at https://github.com/cbm755/cpmatrices
} 
and keypoint detection.

\subsubsection{Diffusion with the Implicit Closest Point Method (ICPM)}

Use the definition of the embedding grid to rewrite the diffusion equation as

$$
\frac{\partial u(t, \mathbf{x})}{\partial t}=\alpha \nabla^{2} u(t, \mathrm{cp}(\mathbf{x}))
$$

where $u$ is the signal, $\nabla^{2}$ is the classical Cartesian defined continuous Laplace operator. The Laplacian is approximated using a linear finite difference scheme of either second order or fourth order centered differences. The discretized Laplacian is applied to each point in $\mathcal{C}$ by taking a linear combination of neighboring points that lie within the differentiation stencil.

Recall that ICPM only operates on $\mathbf{x}_{i} \in \mathbb{R}^{3}$ that lie within a narrow band around the original surface. The widthof this narrow band is [96]

$$
\beta=\delta \sqrt{(d-1)\left(\frac{p+1}{2}\right)^{2}+\left(\frac{l}{2}+\frac{p+1}{2}\right)^{2}}
$$

where $\delta$ is the discretized spacing of the embedding Cartesian grid, $d$ is the dimension of the surface $(d=3$ for this work), $l$ is the order of the Laplacian stencil, and $p$ is the order of the interpolation scheme used to find the values of $u(\operatorname{cp}(\mathbf{x}))$.

Suppose at time $t_{n}>t_{0}$, some diffusion has occurred and $u\left(t_{n}, \mathbf{x}\right)$ has a new value. The value at $u\left(t_{n}, \mathbf{x}\right)$ also should have the same value as $u\left(t_{n}, \mathrm{cp}(\mathbf{x})\right)$. Due to numerical accuracy of the embedding scheme and discretization, that may not always be true for all $t_{n}$. Therefore, the value at 
$u\left(t_{n}, \mathrm{cp}(\mathbf{x})\right)$ is interpolated at each time step using barycentric Lagrange interpolation [98]. Since neither the grid nor the surface are moving, the interpolation weights are precomputed and stored in the closest point extension matrix $\mathbf{E}_{\mathrm{cp}}$

Discretization of Eq. 3.1 begins with stacking the signal values of $\mathcal{U}_{\mathcal{B}}$ into the vector $\mathbf{u}$. Also denote the Cartesian Laplacian as $\mathbf{L}_{\mathfrak{C}}$. The resulting semi-discrete system is stabilized - without impacting the solution consistency - by removing the redundant mapping of $u\left(\mathrm{cp}\left(\mathrm{x}_{i}\right)\right.$ for the Cartesian Laplacian's center points [1]. The stable Laplacian is written as

$$
\mathbf{L}=\operatorname{diag}\left(\mathbf{L}_{\mathcal{C}}\right)+\left(\mathbf{L}_{\mathcal{C}}-\operatorname{diag}\left(\mathbf{L}_{\mathcal{C}}\right)\right) \mathbf{E}_{\mathrm{cp}}
$$

where $\mathbf{L}_{\mathcal{C}}$ is size $m_{\mathcal{B}} \times\left(m_{\mathcal{B}}+m_{\mathcal{G}}\right)$ and $\mathbf{E}_{\mathrm{cp}}$ is size $\left(m_{\mathcal{B}}+m_{\mathcal{G}}\right) \times m_{\mathcal{B}}$ yielding $\mathbf{L}$ of size $m_{\mathcal{B}} \times m_{\mathcal{B}}$. Then Eq. 3.3 written semi-discretely as

$$
\frac{\partial \mathbf{u}_{\mathcal{B}}}{\partial t}=\alpha \mathbf{L} \mathbf{u}_{\mathcal{B}}
$$

where only $\mathcal{U}_{\mathcal{B}}$ is being diffused. Macdonald and Ruuth suggested using a second or fourth order backward differentiation formula (BDF) to discretize Eq. 3.6, but we have found that first order BDF is sufficiently accurate. BDF1 yields

$$
\mathbf{u}_{\mathcal{B}}\left(t_{n+1}\right)=\left(\mathbf{I}-\alpha \tau_{n} \mathbf{L}\right)^{-1} \mathbf{u}_{\mathcal{B}}\left(t_{n}\right)
$$

where $\mathbf{I}$ is the identity matrix the same size as $\mathbf{L}$ and $\tau_{n}$ is the discrete time step such that

$$
\tau_{n}=t_{n+1}-t_{n}
$$


The generalized minimal residual method (GMRES) and the bi-conjugate gradient method (BICG) or its variants [99] efficiently implement numerical solutions to Eq. 3.7.

For future use in constructing a scale-space, we require the signal values at the mesh vertex locations contained in $\mathcal{V}$. Analogous to the interpolation of closest point signal values, the vertex signal values are interpolated using barycentric Lagrange interpolation. Since both the mesh and grid are spatially fixed, the weight are precomputed and stored in the vertex extension matrix $\mathbf{E}_{\mathbf{v}}$. Recovering the signal values $\mathcal{U}$ at any time of interest occurs as

$$
\mathbf{u}\left(\mathbf{v}, t_{n+1}\right)=\mathbf{E}_{\mathbf{v}} \mathbf{u}_{\mathcal{B}}\left(t_{n+1}\right)
$$

where $\mathbf{E}_{\mathbf{v}}$ is size $m_{\mathbf{v}} \times m_{\mathcal{B}}$.

\subsubsection{Mesh Diffusion Examples}

We are interested in testing the qualitative accuracy of diffusion using ICPM to that of 2D Gaussian diffusion; however, it is difficult to diretly draw conclusions between 2D Gaussian diffusion and 3D diffusion on an arbitrary mesh. Fig. 3.3 compares a general mesh with varying connectivity and average edge length to a consistent planer mesh. We compare 2D Gaussian image diffusion to that of 3D diffusion on a planer mesh before applying diffusion to general 3D meshes.

Two examples are shown for an impulse and a general monochrome image. For both examples, the $2 \mathrm{D}$ Gaussian is discretized with a $5 \times 5$ window 

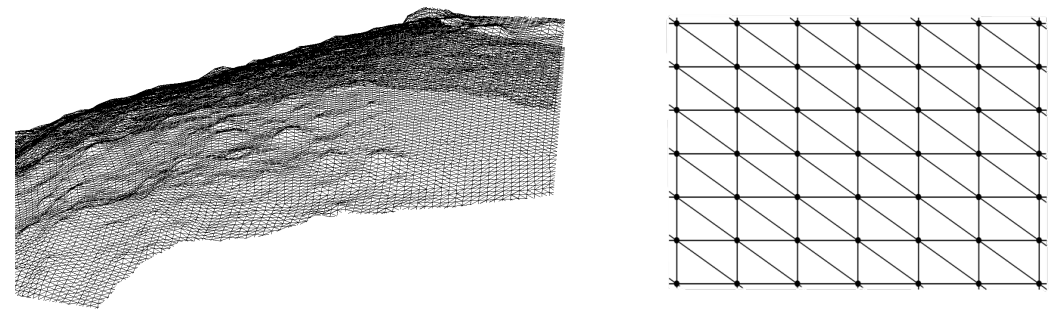

Figure 3.3: A general mesh compared to the simplicity of a planer mesh.

with a standard deviation $\sigma=0.75$. Explicit meshes are constructed with an identical size to that of the image, where each vertex directly corresponds to a pixel. Implicit surfaces constructed using the CPR have different grid spacing for each example. For impulse diffusion, the grid spacing is $\delta=0.25$ and has a time step identical to 2D Gaussian diffusion. For monochrome image diffusion, the grid spacing is $\delta=0.5$ and has a time step of $t=\delta / 4$. Both examples use a second order Laplacian stencil $(l=2)$ and a third order barycentric Lagrange interpolation $(p=3)$.

\subsubsection{Diffusion of an Impulse}

Results for 2D Gaussian image diffusion are shown in Fig. 3.4. Notice that the pixel values shown by the red dots lie on the predicted Gaussian curve. The same response is sought for implicit surface diffusion. Results for the 3D planar mesh diffusion using an implicit surface are shown in Fig. 3.5. Notice that the vertex values shown by the red dots lie near the predicted Gaussian curve for $\sigma=1.5$, and become more numerically accurate as $t$, and correspondingly $\sigma$ grow larger. Numerical interpolation of the grid points us- 

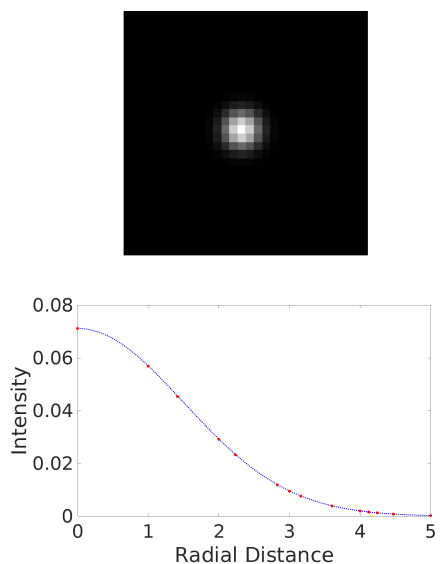

(a) $\sigma_{5}=1.500$
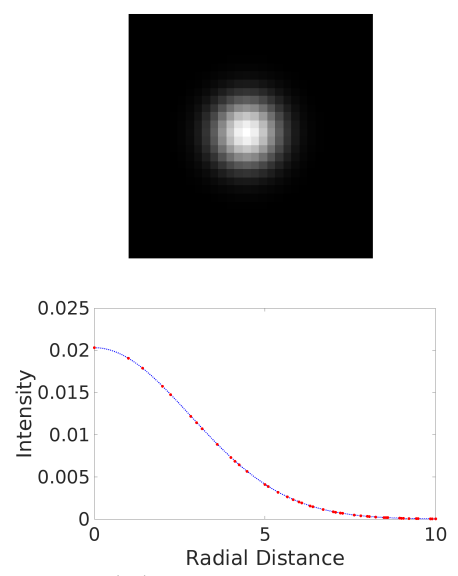

(b) $\sigma_{15}=2.806$
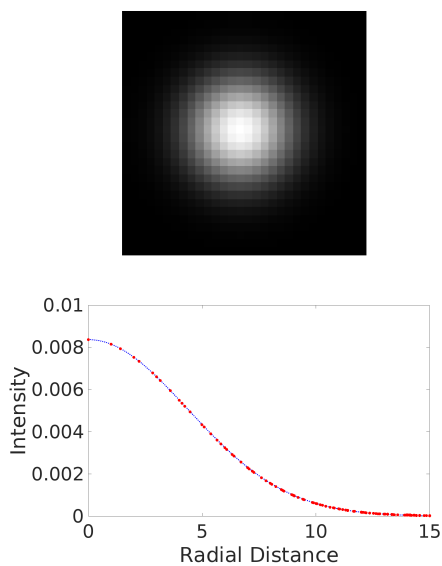

(c) $\sigma_{35}=4.373$

Figure 3.4: Gaussian diffusion of an impulse on a 2D image. Red dots are the numeric values of the intensity as a function of radial distance from the center. The blue dotted line is the Gaussian distribution at that scale. Notice the difference in the abscissa limits.

ing barycentric Lagrange interpolation occasionally yields negative values, but always on the order of machine precision. These negative values are reassigned to zero, but the effect may be mitigated by using another interpolation method, such as weighted essentially non-oscillatory (WENO) ${ }^{3}[100,101]$. Notice that the diffusion approaches Gaussian as the time increases (left to right). It was also noticed that diffusion approaches Gaussian earlier in time as the embedding grid spacing becomes smaller (not shown).

\footnotetext{
${ }^{3}$ Weights for the WENO scheme depend on the data and must be recomputed each time.
} 

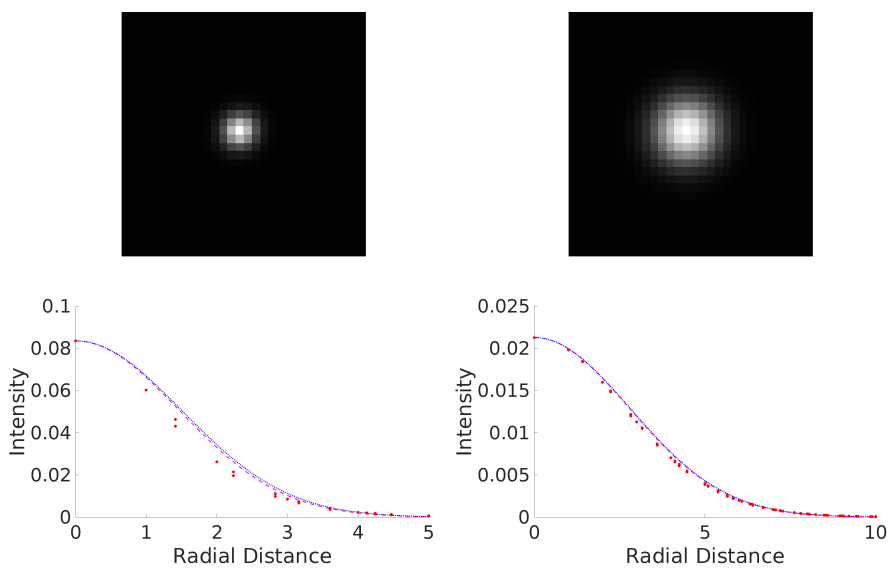

(a) $\sigma_{5}=1.500$

(b) $\sigma_{15}=2.806$
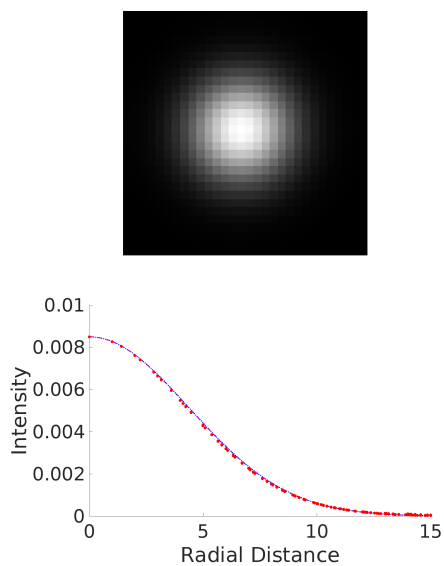

(c) $\sigma_{35}=4.373$

Figure 3.5: Impulse diffusion on an implicit planar mesh for comparison to Fig. 3.4. Red dots are intensity as a function of radial distance from the center. Dotted blue line is the Gaussian distribution with the noted standard deviation. Notice the difference in the abscissa limits.

\subsubsection{Diffusion of a Monochrome Image}

Diffusion of a monochrome image of Comet $67 \mathrm{P} /$ Churyumov-Gerasimenko ${ }^{4}$ (resampled to a size of $407 \times 564$ pixels) is shown in Fig. 3.6 with the first row showing 2D image Gaussian diffusion and the second row showing 3D implicit surface diffusion. Upon visual inspection, any difference between the two methods is imperceptible. Therefore, discretized diffusion on an implicit surface using ICPM offers an accurate method for solving the diffusion equation, and thus for constructing a scale-space.

\footnotetext{
${ }^{4}$ Original image provided as an IMG file in the archive delivery from: ESA/Rosetta/MPS for OSIRIS Team MPS/UPD/LAM/IAA/SSO/INTA/UPM/DASP/IDA
} 

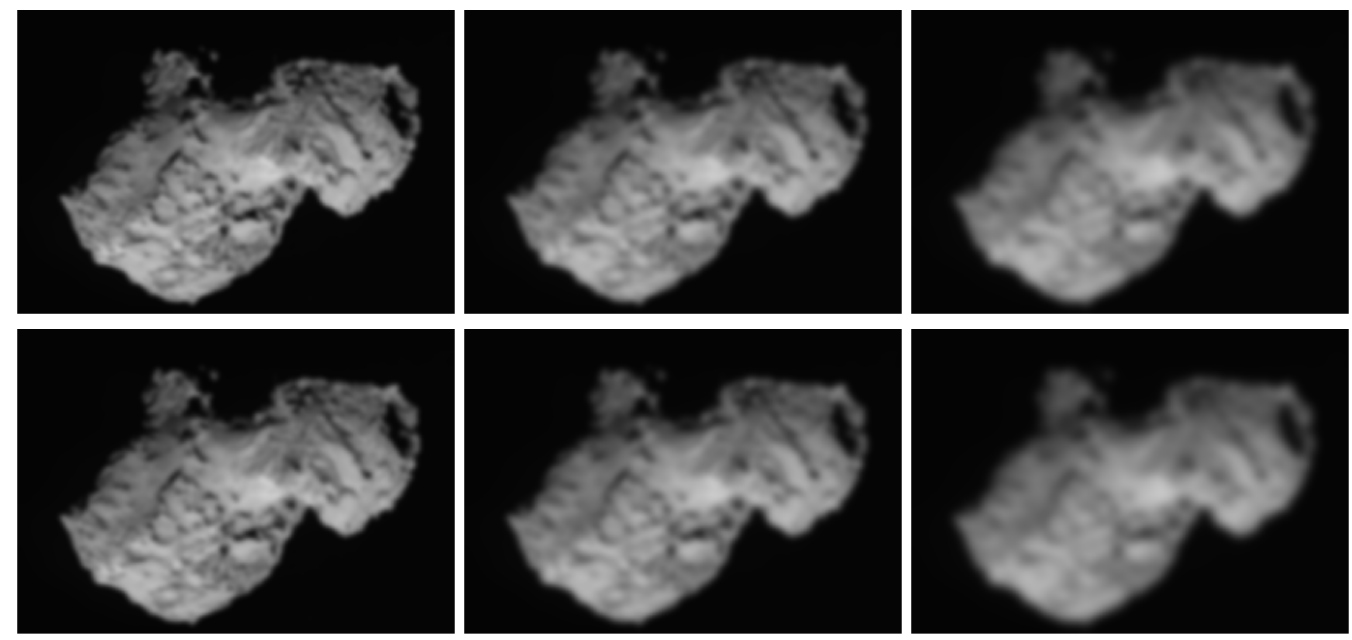

(d) $\sigma_{50}=3.54$

(e) $\sigma_{100}=5.0$

(f) $\sigma_{200}=7.07$

Figure 3.6: Diffusion of a monochrome gray image of Comet 67P/CG. Top row is 2D Gaussian diffusion. Bottom row is 3D implicit surface diffusion with DI.

\subsubsection{Comments}

The ICPM is extremely accurate for solving PDEs on arbitrary surfaces. Be embedding an general surface in a Cartesian grid, classic definitions of the gradient, divergence, and Laplacian are utilized. This embedding, however, also increases the dimensionality of the data set beyond the original surface size. Depending on the order of the Laplacian stencil and the interpolation scheme, the number of points of the implicit surface is, at minimum, twice as large as the explicit surface. Affects of this size increase carries also extends to constructing $\mathbf{L}$ and thus the inversion necessary in Eq. 3.7. Methods for solving diffusion on general surfaces should be both accurate and quick for use in real-time engineering applications. While DI was considered in [58], it is 
not quick, and thus no longer considered in this manuscript.

\subsection{Gaussian Diffusion on Implicit Surfaces (GI)}

While the CPR was developed to solve a variety of PDEs on general surfaces, scale-space construction depends on solving the diffusion equation. While the diffusion equation maybe solved with numerical integretration methods (e.g. BDF or Runge-Kutta), the characteristic solution is the Gaussian. CPR implicit defines general surfaces with points in a Cartesian grid, on which the Gaussian kernel is well-understood. Another work associated with the development of the CPR utilized the Gaussian for the diffusion of constrained curves on surfaces [102]. We utilize the CPM to solve the diffusion equation on implicit surfaces for the purpose of scale-space construction.

\subsubsection{Diffusion with the Closest Point Method (CPM)}

Recall the definition of the embedding grid $\overline{\mathcal{M}}$ to construct the individual components of the discrete diffusion equation. Begin by stacking the signal $\mathcal{U}_{\mathcal{B}}$ into the vector $\mathbf{u}_{\mathcal{B}}$. Recall that CPM only solves one step of diffusion on the narrow band followed by an immediate reevaluation of the grid signal. Using barycentric Lagrange interpolation [98], the weights are precomputed

and stored in the closest point extension matrix $\mathbf{E}_{\mathrm{cp}}$. This extension matrix interpolates $u(\operatorname{cp}(\mathbf{x}))$ in each step of diffusion. 
Denote the Cartesian Gaussian as $\mathbf{G}_{\mathcal{e}}$ such that

$$
\mathbf{G}_{\mathcal{C}(i j)}= \begin{cases}\frac{1}{\sigma^{3}(2 \pi)^{3 / 2}} \exp \left(\frac{-\left\|\mathbf{x}_{i}-\mathbf{x}_{j}\right\|^{2}}{2 \sigma^{2}}\right) & \text { if }\left\|\mathbf{x}_{i}-\mathbf{x}_{j}\right\| \leq m_{\sigma} \sigma \\ 0 & \text { otherwise }\end{cases}
$$

where $\sigma$ is the standard deviation of the Gaussian and $\|\cdot\|$ is the Euclidean distance metric acting on the embedding grid points. Then the Gaussian matrix for diffusion on implicit surfaces is

$$
\mathbf{G}=\mathbf{G}_{\mathrm{c}} \mathbf{E}_{\mathrm{cp}}
$$

where $\mathbf{G}_{\mathcal{C}}$ is size $m_{\mathcal{B}} \times\left(m_{\mathcal{B}}+m_{\mathcal{G}}\right)$ and $\mathbf{E}_{\mathrm{cp}}$ is size $\left(m_{\mathcal{B}}+m_{\mathcal{G}}\right) \times m_{\mathcal{B}}$. The discrete Gaussian is normalized to have unit row sum. Return to Eq. 3.3 and solve using the discrete Gaussian as

$$
\mathbf{u}_{\mathcal{B}}\left(\sigma_{n+1}\right)=\mathbf{G u}_{\mathcal{B}}\left(\sigma_{n}\right)
$$

where $\mathbf{G}$ is size $m_{\mathcal{B}} \times m_{\mathcal{B}}$.

For the purpose of scale-space construction, we must calculated and store the values of $\mathcal{U} \in \mathcal{M}$ at particular time steps. This is accomplished using the vertex extension matrix $\mathbf{E}_{\mathbf{v}}$. Recovering the signal $\mathcal{U}$ is performed as

$$
\mathbf{u}\left(\mathbf{v}, \sigma_{n+1}\right)=\mathbf{E}_{\mathbf{v}} \mathbf{u}_{\mathcal{B}}\left(\sigma_{n+1}\right)
$$

where $\mathbf{E}_{\mathbf{v}}$ has size $m_{\mathbf{v}} \times m_{\mathcal{B}}$.

\subsubsection{Determining the Bandwidth}

Only a narrow band around the original surface is used for CPR. The bandwidth was defined in Eq. 3.4 for a Laplacian stencil, but here we use the 
Gaussian. We propose a modification of the bandwidth equation to make it a function of the Gaussian standard deviation and the support region as

$$
\beta=\delta \sqrt{(d-1)\left(\frac{p+1}{2}\right)^{2}+\left(\frac{m_{\sigma} \sigma}{\delta}+\frac{p+1}{2}\right)^{2}}
$$

where $\delta$ is the discretized spacing of the embedding Cartesian grid, $d$ is the dimension of the surface ( $d=3$ for this work), $p$ is the order of the interpolation scheme, $\sigma$ is the standard deviation or the Gaussian, and $m_{\sigma}$ is the number of standard deviations used in the support window. While $\delta$ and $p$ are already known, we still need to determine values for $m_{\sigma}$ and $\sigma$ to provide sufficiently accurate diffusion while maintaining a small embedding grid.

\subsubsection{Determining the Kernel Window and Width}

Using the method DI from Section 3.2, the Laplacian stencil only uses in-plane points. The Gaussian is defined radially and thus requires many more embedding points for construction. It is of interest to determine the minimum required bandwidth for insight into the memory needed to use the GI method.

Suppose we extrapolate the square window of support from $2 \mathrm{D}$ image diffusion to a cubic window for Gaussian diffusion on 3D implicit surfaces. Suppose the cube has a side-length of $2 r$. Since the Gaussian is defined radially, then statistically, all of its mass exist within the sphere of radius $r$. There are points bounded by the cube - but outside the sphere - that statistically have no mass. Thus we can constrain the support window size and thus the number of points in the embedding grid. 
While counting the number of lattice points within a sphere is a still an open ended problem ${ }^{5}$, we may place an approximate upper bound through the ratio of sphere volume to cubic volume as

$$
m_{s} \lesssim \frac{(4 / 3) \pi r^{3}}{8 r^{3}} m_{c}=\frac{\pi}{6} m_{c}
$$

where $m_{c}$ is the number of points in the cube and $m_{s}$ is the number of points in the sphere. We are always guaranteed to have fewer points within the sphere than the cube in $\mathbb{R}^{3}$. 6

Determining the radius $r$ of the sphere such that $r=m_{\sigma} \sigma$ will regulate the number of points needed for the embedding grid. The higher dimensional erf [103] is analyzed to determine the number of standard deviations used in the support window $m_{\sigma}$. While it is common to use $3 \sigma$ support in $1 \mathrm{D}$ data to cover $99.7 \%$ of the weight, this only accounts for $97 \%$ of the weight in $3 \mathrm{D}^{7}$ We suggest using $m_{\sigma}=4$ to account for $99.8 \%$ of the weight and to maintain a small embedding grid.

The standard deviation for the implicit Gaussian is of critical importance for maintaining sparsity of $\mathbf{G}_{\mathrm{e}}$ and is fundamentally tied to the selection of the embedding grid spacing, $\delta$. We suggest that $\sigma \leq \delta$ so that $\mathbf{G}_{\mathcal{e}}$ maintains sparsity and thus efficiency.

\footnotetext{
${ }^{5}$ It is an extension of the Gauss Circle Problem

${ }^{6}$ Spheres are not bounded by tight fitting cubes for $d \geq 9$.

${ }^{7}$ Error function values may be obtained using Matlab function $\operatorname{chi} 2 c d f\left(m_{\sigma}^{2}, d\right)$.
} 


\subsubsection{Comments}

Constructing a Gaussian on on implicit surfaces for implementing the diffusion equation eliminates the need to invert a large sparse matrix, but comes with its own limitations. The discrete Gaussian requires more points within the stencil than the Laplacian, resulting in larger implicit surface. The number of points in the implicit surface is, at minimum, four times larger than the explicit surface. These additionally points make the GI method unsuitable for real-time engineering applications. We no longer consider this method in this manuscript.

\subsection{Discretized Diffusion on Explicit Surfaces (DE)}

Literature contains many examples of mesh description and manipulation using discrete LBOs. While many of the method focus on the movement of mesh vertex locations [104, 105, 65], there has been an recent interest in the diffusion of signals on surfaces $[57,59]$. The main difference between these publications is (1) the selection of LBO and (2) the selection of time steps for scale-space construction. This section will cover the discrete implementation of signal diffusion on surfaces and a discussion on LBO selection. Chapter 4 will discuss the time step selection.

\subsubsection{Diffusion with discrete Laplacians}

To implement the diffusion equation of Eq. 3.1 on discrete meshes, begin by stacking the signal $\mathcal{U}$ into the vector $\mathbf{u}$. Also discretize $\nabla_{\mathcal{S}}^{2}$ to yield 
the semi-discrete diffusion equation

$$
\frac{\partial \mathbf{u}}{\partial t}=\alpha \mathbf{L u}
$$

where $\mathbf{u}$ is a vector of length $m_{\mathbf{v}}$ and $\mathbf{L}$ is the discrete LBO of size $m_{\mathbf{v}} \times m_{\mathbf{v}}$. In principal, the exact solution is

$$
\mathbf{u}(t)=\exp (\alpha t \mathbf{L}) \mathbf{u}\left(t_{0}\right)
$$

where the matrix exponential is defined by the convergent power series

$$
\exp (\alpha t \mathbf{L})=\sum_{i=0}^{\infty} \frac{\alpha^{i} t^{i}}{i !} \mathbf{L}^{i}
$$

This method is computationally expensive for large matrices, but many approximation methods exist [106], the most popular of which is the scaling and squaring method using Padé approximations [107, 108]. Calculating the action of the matrix exponential leads to other popular methods of approximating $\exp (\alpha t \mathbf{L}) \mathbf{u}$, such as using Krylov subspaces [109, 110], interpolation of Leja points [111, 112], and truncated Taylor series [113]. Based on typical mesh sizes and the current state-of-the-art algorithms, we found that these methods are currently too computationally expensive to use. We instead look to first-order linearized methods.

Taking the Taylor series expansion of Eq. 3.18 to first order is equivalent to linearizing the left-hand-side (LHS) of Eq. 3.16 using the forward-intime Euler method. This requires small time steps pursuant to the CourantFriedrichs-Lewy (CFL) condition [114] or else it is notably unstable. Using 
Table 3.2: Convergence types for the three tested LBOs listed in order from strongest to weakest (top to bottom).

\begin{tabular}{ll}
\hline \hline LBO & Convergence \\
\hline Mesh [115] & $\mathrm{L}^{\infty}[116]$ \\
Umbrella [117] & in distribution [116] \\
Cotangent [118] & in probability [119] \\
\hline \hline
\end{tabular}

the backward-in-time Euler method instead leads to

$$
\mathbf{u}\left(t_{n+1}\right)=\left(\mathbf{I}-\alpha \tau_{n} \mathbf{L}\right)^{-1} \mathbf{u}\left(t_{n}\right)
$$

where $\tau_{n}$ is the time step

$$
\tau_{n}=t_{n+1}-t_{n}
$$

Eq. 3.19 is unconditionally stable, but requires inverting a large sparse matrix, and accuracy still depends on the magnitude of the time step $\tau_{n}$.

Eq. 3.19 is also known as the first order backward differentiation formula (BDF1) used in $[104,57]$. Higher order methods of BDF or Runge-Kutta may also be used, but we found that first order is sufficiently accurate. The generalized minimal residual method (GMRES) and the bi-conjugate gradient method (BICG) or its variants [99] efficiently implement numerical solutions to Eq. 3.19.

\subsubsection{Laplace-Beltrami Operators (LBO)}

The Laplace-Beltrami operator (LBO) is the extension of the Laplace operator to functions defined on surfaces in Euclidean space. It is numerically 
constructed in sparse matrix form for discrete meshes. The most popular implementations are the umbrella, cotangent, and mesh operators. They differ by the weighting scheme, which is how they acquire their names. Each exhibit different convergence properties as listed in Table 3.2 in descending order of strength. It is expected that stronger convergence leads to better accuracy and higher repeatability. Normalized LBOs have zero-sum rows with negative diagonal values, positive values in the row elements defined by the support region, and zeros everywhere else. The matrix construction is given by

$$
\mathbf{L}=\mathbf{W}-\mathbf{D}
$$

where $\mathbf{L}$ is the $\mathbf{L B O}, \mathbf{W}$ is the off-diagonal weighting matrix, and $\mathbf{D}$ is the diagonal matrix defined as

$$
\mathbf{D}_{i i}=\sum_{j} \mathbf{W}_{i j}
$$

where Figure 3.7 shows examples of their sparsity. Sparsity offers graphical insight into the LBO surface representation and its relative computational expense. The umbrella and cotangent LBOs will be computationally faster to implement, but the mesh LBO offers a more accurate representation of the surface.

\subsubsection{Umbrella Laplace Operator}

The umbrella operator is a combinatorial construction of the LBO [117, 120]. Its construction solely depends on the graph connectivity of the mesh vertices without regard for the geometry of the underlying mesh. The most 

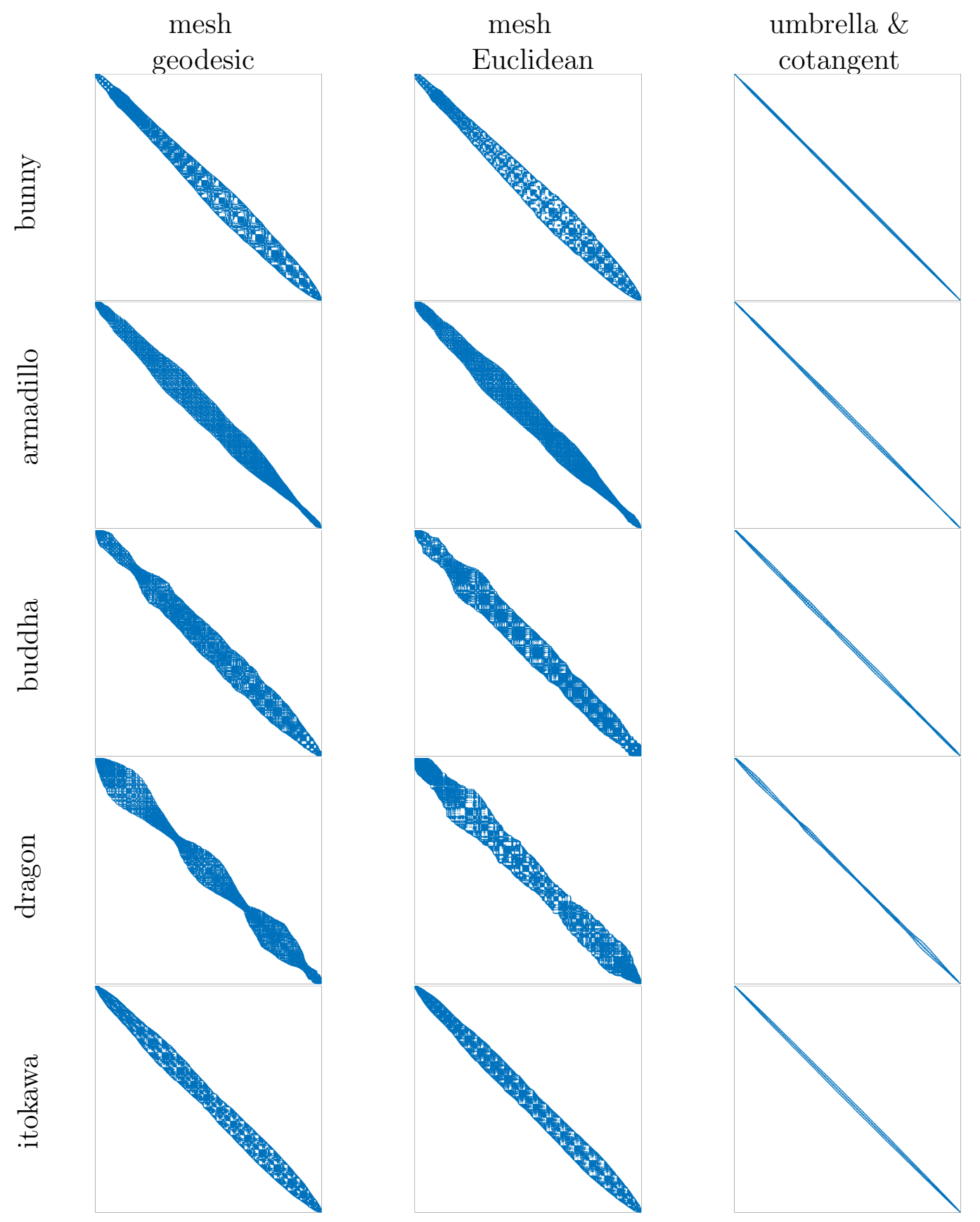

Figure 3.7: Visualization of the LBO sparsity for various mesh models. Rows are categorized by model, while columns are categorized by LBO. Umbrella and cotangent LBO have the same sparsity structure. 
common umbrella weighting scheme, and the one which we use in this paper, uses the cardinality of the first-ring neighborhood

$$
\mathbf{W}_{i j}= \begin{cases}1 /\left|\mathcal{N}\left(\mathbf{v}_{i}\right)\right| & \text { if } \mathbf{v}_{j} \in \mathcal{N}\left(\mathbf{v}_{i}\right) \\ 0 & \text { otherwise }\end{cases}
$$

where $\mathcal{N}\left(\mathbf{v}_{i}\right)$ is the first-ring neighborhood of vertices connected to $\mathbf{v}_{i}$. The weights also could be a function of the edge length between neighboring vertex locations or a ratio of the face areas of neighboring faces [105, 120]. The underlying assumption is that all vertices are homogeneously sampled from the surface and have uniform connectivity - an assumption rarely satisfied in practice. The umbrella operator presented here is identical for variously shaped neighborhoods as long as they have identical connectivity.

Belkin and Niyogi showed that the umbrella operator exhibits convergence in probability if the points are randomly sampled from a uniform distribution of the underlying manifold [116]. The right column Fig. 3.7 shows the sparse structure of the umbrella LBO for a variety of common mesh models.

\subsubsection{Cotangent Laplace Operator}

The cotangent operator ${ }^{8}$ is the most common form of the LBO. It is also constructed on the graph connectivity of the mesh, but includes some information regarding the shape of the faces. Specifically, it uses the cotangent of the angles that lie opposite of the connecting edge between two faces.

\footnotetext{
${ }^{8}$ Code may be found at https://github.com/areslp/matlab/tree/master/MeshLP.
} 
Variety in the cotangent weighting schemes arises from the normalization factor with examples being a constant [121], the summation of face areas of the first-ring neighborhood [104], or a mixed summation of face areas depending on the obtuseness of the faces [118]. We utilize the mixed area normalized cotangent defined as [118]

$$
\mathbf{W}_{i j}= \begin{cases}\frac{1}{2 A_{m}\left(\mathbf{v}_{i}\right)}\left(\cot \left(\alpha_{i}\right)+\cot \left(\beta_{j}\right)\right) & \text { if } \mathbf{v}_{j} \in \mathcal{N}\left(\mathbf{v}_{i}\right) \\ 0 & \text { otherwise }\end{cases}
$$

where $\alpha_{i}$ and $\beta_{j}$ are the angles opposite the common edge $e_{i j}$ between $\mathbf{v}_{i}$ and $\mathbf{v}_{j}$, and $A_{m}\left(\mathbf{v}_{i}\right)$ is given in [118] which considers both obtuse and non-obtuse triangular faces. Fig. 3.8 shows an example of the mixed area cotangent LBO. The cotangent LBO also assumes that all vertices are homogeneously sampled from the surface and have uniform connectivity.

Wardetzky shows that the cotangent LBO exhibits convergence in distribution, but not in $\mathrm{L}^{2}$ [119]. This is the weakest form of convergence among the three tested LBOs. While the values may be different, the sparse structure of the cotangent LBO is identical to that of the umbrella LBO because both are constructed on connectivity.

\subsubsection{Mesh Laplace Operator}

The mesh Laplacian ${ }^{8}$ is the most recently developed version of the LBO, originally for meshed surfaces [115], but also extended to point clouds ${ }^{9}$ [122]. It is distinct from the other LBO operators because it is defined by distance

\footnotetext{
${ }^{9}$ Code may be found at https://github.com/mruan/pcdlaplace
} 


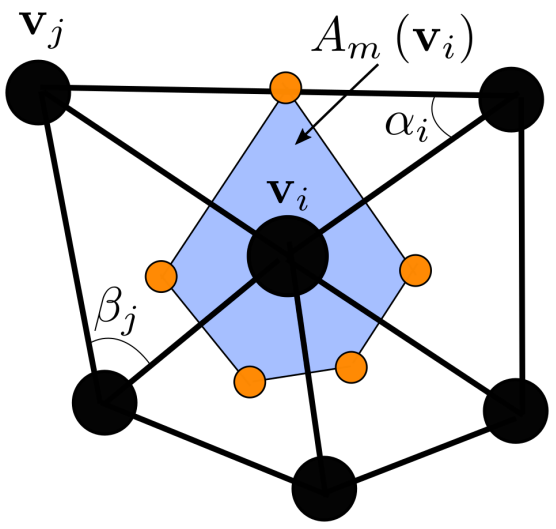

Figure 3.8: Graphical representation of the mixed area cotangent LBO.

between neighboring vertices instead of connectivity. The weighting matrix is defined as

$$
\mathbf{W}_{i j}= \begin{cases}\frac{A\left(\mathbf{v}_{j}\right)}{4 \pi h^{2}} \exp \left(\frac{-\left\|\mathbf{v}_{i}-\mathbf{v}_{j}\right\|^{2}}{4 h}\right) & \text { if }\left\|\mathbf{v}_{i}-\mathbf{v}_{j}\right\|<\rho \sqrt{h} \\ 0 & \text { otherwise }\end{cases}
$$

where $h$ is a positive quantity describing the support region, $A\left(\mathbf{v}_{i}\right)$ is the area weight defined as

$$
A\left(\mathbf{v}_{i}\right)=\sum_{f_{j} \mid \mathbf{v}_{i} \in f_{j}} \frac{A\left(f_{j}\right)}{\left|f_{j}\right|}
$$

$\left|f_{i}\right|$ is the cardinality of the face (e.g. $\left|f_{j}\right|=3$ for triangular faces), and the summation is carried out on all faces $f_{j}$ containing vertex $\mathbf{v}_{i}$. The exponent uses the geodesic distance metric; however, Belkin shows that the Euclidean distance is accurate to third order for dense meshes [115]. The neighborhood size $\rho$ is a user-defined scalar that, when multiplied by $h$, defines the Gaussian support. Default values are $h=\bar{e}^{2}$ and $\rho \in[3,6]$, and assuming that $\alpha=1$. An optimal choice of $h=\bar{e}^{2 / 5}$ was suggested in [122], but we have not witnessed significant improvement over the default value. 
Several authors have shown the mesh LBO to have point-wise convergence $\mathrm{L}^{\infty}$, and thus also $\mathrm{L}^{2}$ for increasingly dense meshes [115, 116, 123]. This is the strongest type of convergence among the three tested LBOs.

Fig. 3.7 shows the sparse structure of the mesh LBO for a variety of common mesh models. The left and center columns show the structure for geodesic and Euclidean distance respectively. Parameters chosen for this representation are $h=\bar{e}^{2}$ and $\rho=4$.

\subsubsection{Effects of Mesh Scaling}

Discrete diffusion of Eq. 3.19 operates in time (i.e. $\tau_{n}$ or $t_{n}$ ), but the LBOs are defined on physical properties such as connectivity, distance, and face areas. We wish to determine a relationship between the diffusion time and the physical scale of the mesh, as related through the LBO. One approach to determine the effect analyzes the orthonormal basis of eigenfunctions that form the heat kernel [124]. We offer a different discussion here.

Both the choice of the LBO and the physical scale of the underlying mesh may affect the amount of diffusion that has occurred at time $t_{n}$. This is immediately evident from an inspection of the three different discrete LBOs.

Suppose, for example, mesh $\mathcal{M}$ was scaled by $\beta$ to create $\mathcal{M}^{\prime}$, such that $\bar{e}^{\prime}=\beta \bar{e}$. Observe that $\mathbf{W}_{i j}^{\prime}$ of the umbrella LBO would remain unchanged, while the cotangent and mesh LBOs would scale such that $\mathbf{W}_{i j}^{\prime}=\left(1 / \beta^{2}\right) \mathbf{W}_{i j}$. As a consequence, mesh scale affects the umbrella LBO differently from the cotangent and mesh LBOs. These two scenarios are now discussed. 
First consider the cotangent and mesh LBOs. The scaling of the mesh - and consequently $\mathbf{W}_{i j}$ - means that the time it takes to achieve the same level of diffusion between a common pair of points is $t_{n}^{\prime}=\beta^{2} t_{n}$. For example, if $\beta>1$, then diffusion will take longer in time for the same amount of signal to transfer between two points on $\mathcal{M}^{\prime}$ as it did on $\mathcal{M}$. These two points are further apart on $\mathcal{M}^{\prime}$, and this larger distance is appropriately represented in $\mathbf{W}_{i j}^{\prime}$. Thus, the cotangent and mesh LBOs naturally consider scale in their construction and no adjustment is necessary when constructing a scale-space.

Now consider the umbrella LBO. This LBO considers only connectivity and $\mathbf{W}_{i j}$ is unaffected by mesh scaling. Consequently, the same amount of diffusion will occur between two points over a given time step regardless of the actual mesh size. Therefore, when using the umbrella operator, the amount of diffusion that occurs at $t_{n}^{\prime}$ in the scaled mesh is equivalent to diffusion occurring at $t_{n}$ in the original mesh. Furthermore, the time advancement of diffusion is independent of the value $\bar{e}$ for the umbrella LBO. This relationship causes local diffusion to occur quicker if $\bar{e}_{i}<\bar{e}$ or slower if $\bar{e}_{i}>\bar{e}$.

\subsubsection{Mesh Diffusion Examples}

Performance evaluations for visual appearances are performed in this section. Our objective is to verify that our discretized diffusion is equivalent to that of Gaussian diffusion on 2D images. Testing begins with an impulse on an image, followed by diffusion of a general image, and finally diffusion of a signal on a general 3D surface. Compare examples of impulse diffusion to 

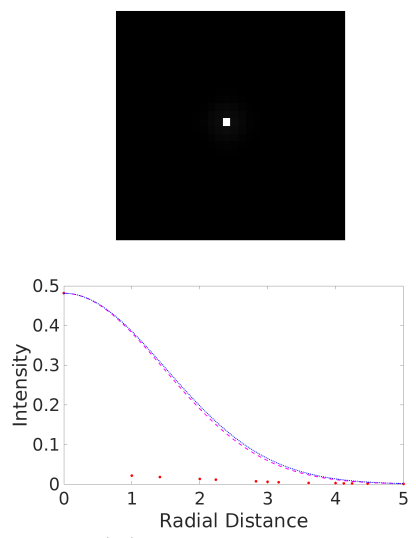

(a) $\sigma_{5}=1.500$
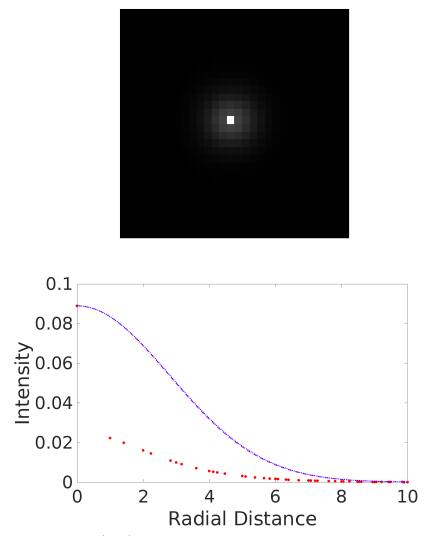

(b) $\sigma_{15}=2.806$
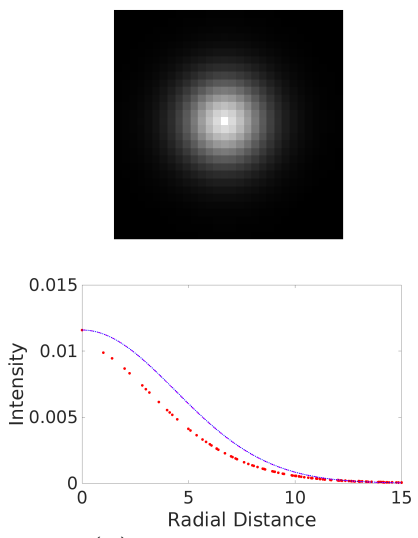

(c) $\sigma_{35}=4.373$

Figure 3.9: Impulse diffusion on an explicit planar mesh using method DE for comparison to Fig. 3.4. Diffusion approaches Gaussian as the number of iterations grows large for DE. Dotted blue line is the Gaussian distribution with the noted standard deviation. Notice the difference in the abscissa limits.

that of Gaussian diffusion in Fig. 3.4.

\subsubsection{Diffusion of an Impulse}

An impulse is placed at the center of an image and diffused with DE using Euclidean mesh LBO. Parameter values are $\rho=3, \sigma=0.75$, and $\bar{e}=1$. Three time steps are selected and shown in Fig. 3.9. Images are normalized by largest intensity for purposes of visualization. The top row shows how the impulse diffuses across the image as the scale grows. The bottom row shows the distribution of intensities as red dots and the exact Gaussian for the given standard deviation. Notice that diffusion approaches Gaussian as the number of steps grows large.

Since the mesh LBO depends on the average edge length $\bar{e}$, we inves- 


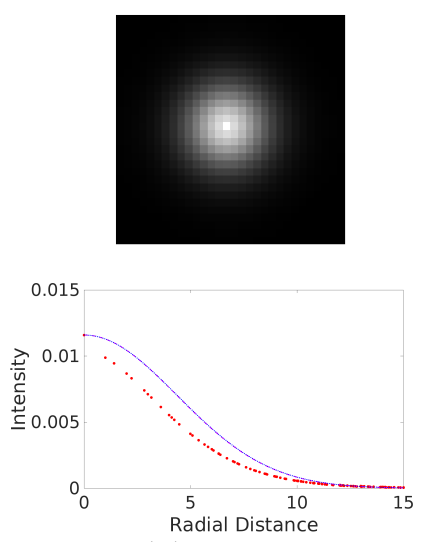

(a) $\bar{e}=1$
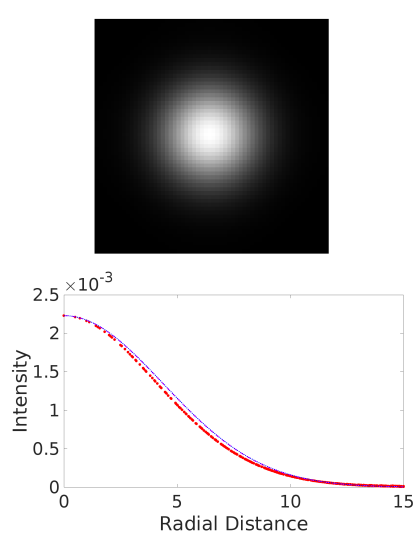

(b) $\bar{e}=0.5$
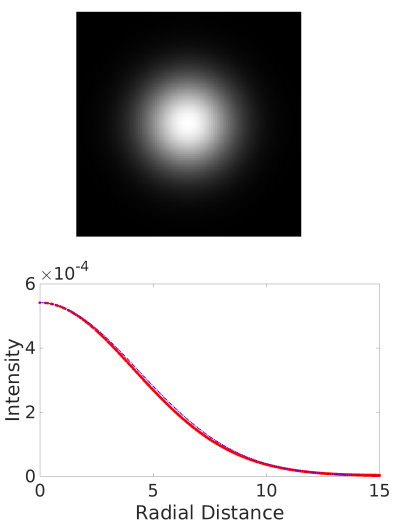

(c) $\bar{e}=0.25$

Figure 3.10: Diffusion approaches Gaussian as the average edge length decreases for DE. These three examples are shown for identical amounts of diffusion $\sigma_{35}=4.373$.

tigate the effect of mesh edge length on diffusion. Three images of the same size are constructed with average edge lengths of $\bar{e}=1, \bar{e}=0.5$, and $\bar{e}=0.25$. Diffusion occurs until the equivalent scale of $\sigma=4.373$ is achieved. The results are shown in Fig. 3.10. Notice that the blurred impulses in the top row are of identical diameter, meaning that the same amount of diffusion has occurred. Accuracy of DE improves as $\bar{e}$ decreases. Also, the size of the mesh LBO increases as $\bar{e}$ decreases since there are more vertices, but sparsity is maintained.

\subsubsection{Diffusion of a Monochrome Image}

Diffusion using DE of a general planar image is compared to that of Gaussian diffusion. A planar surface is constructed with an identical size to an image of Comet $67 \mathrm{P} / \mathrm{CG}$ with the image signal overlaid directly on the 

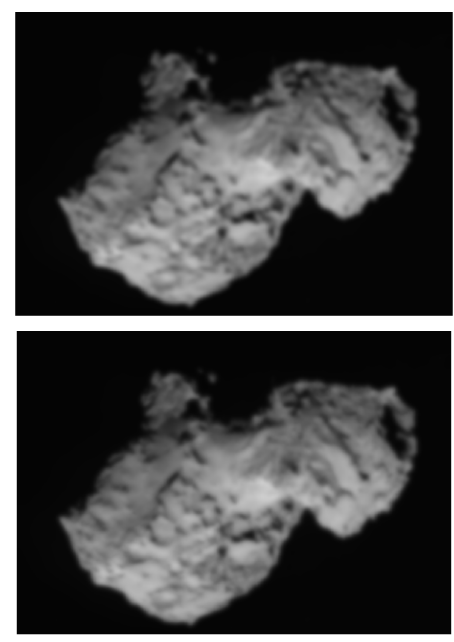

(a) $\sigma_{50}=3.54$
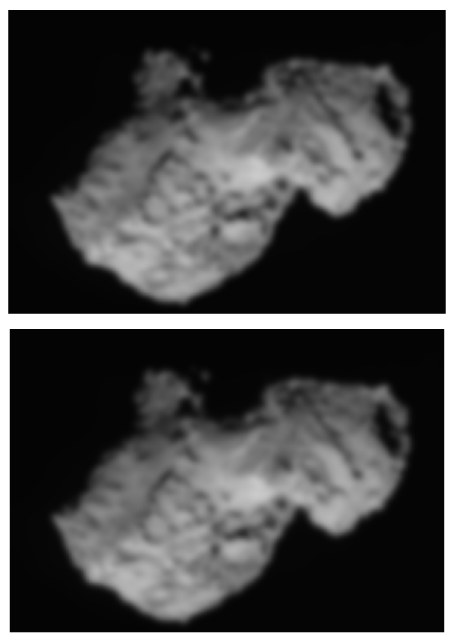

(b) $\sigma_{100}=5.0$
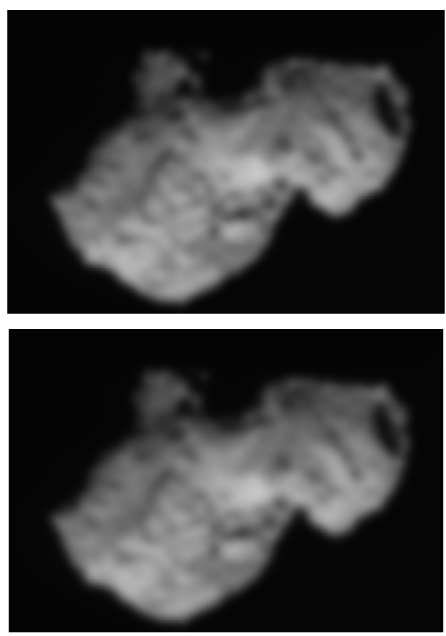

(c) $\sigma_{200}=7.07$

Figure 3.11: Diffusion of a monochrome gray image of Comet 67P/CG. Top row is $2 \mathrm{D}$ Gaussian diffusion. Bottom row is method DE using the mesh LBO.

mesh vertices. Gaussian diffusion of the image is compared to method DE using the Euclidean mesh LBO. Parameters are $\rho=3$ and $\sigma=0.5$ with $\bar{e}=1$. Diffusion results are shown in Fig. 3.11. Notice the visual similarities between Gaussian diffusion and method DE. These examples demonstrate that method DE yields visually similar results to that of Gaussian diffusion.

\subsubsection{Diffusion of a 3D Model}

Demonstrating thee equivalence of diffusion on planar meshes permits the expansion to testing signal diffusion on general 3D meshes. Since no commonly accepted general surface diffusion method exists, we compare the diffusion of each of the LBOs on four models from the Stanford Scanning Repository [125] and on a model of Itokawa [126]. Consider each model as a Lambertian 
surface with a pixel intensity as a function of lighting direction. Diffusion using method DE is performed on this Lambertian reflectance using each of the four LBOs.

Diffusion results are shown throughout Fig. 3.12, Fig. 3.13, Fig. 3.14, Fig. 3.15, and Fig. 3.16 for the scales of $\sigma=1.5 \bar{e}, \sigma=3.35 \bar{e}$, and $\sigma=6.71 \bar{e}$. Notice that the model vertices remain fixed while it is the signal along the vertices that is being diffused. Diffusion using the mesh LBO with either geodesic or Euclidean distances yield visually similar results. Diffusion with cotangent LBO appear most similar to mesh LBO but still with distinct differences. Diffusion with umbrella LBO yields an amount of diffusion inversely proportion to the average edge length. The umbrella LBO yields more diffusion than the other LBOs on the model buddah which has an average edge length of $\bar{e}=5.21$. The umbrella LBO yields less diffusion than the other LBOs on the model bunny which has $\bar{e}=1.0$.

This analysis shows that diffusion with the umbrella LBO yields results dependent on the mesh average edge length. Diffusion with mesh LBO using Euclidean distance is visually equivalent to, and thus an acceptable approximation of, mesh LBO with geodesic distance. The cotangent LBO yeilds diffusion most similar to the mesh LBO.

\subsection{Gaussian Diffusion on Explicit Surfaces (GE)}

With the wide popularity and success of SIFT [49] comes a bevy of diffusion implementations on surfaces using discrete Gaussians. Most of the 

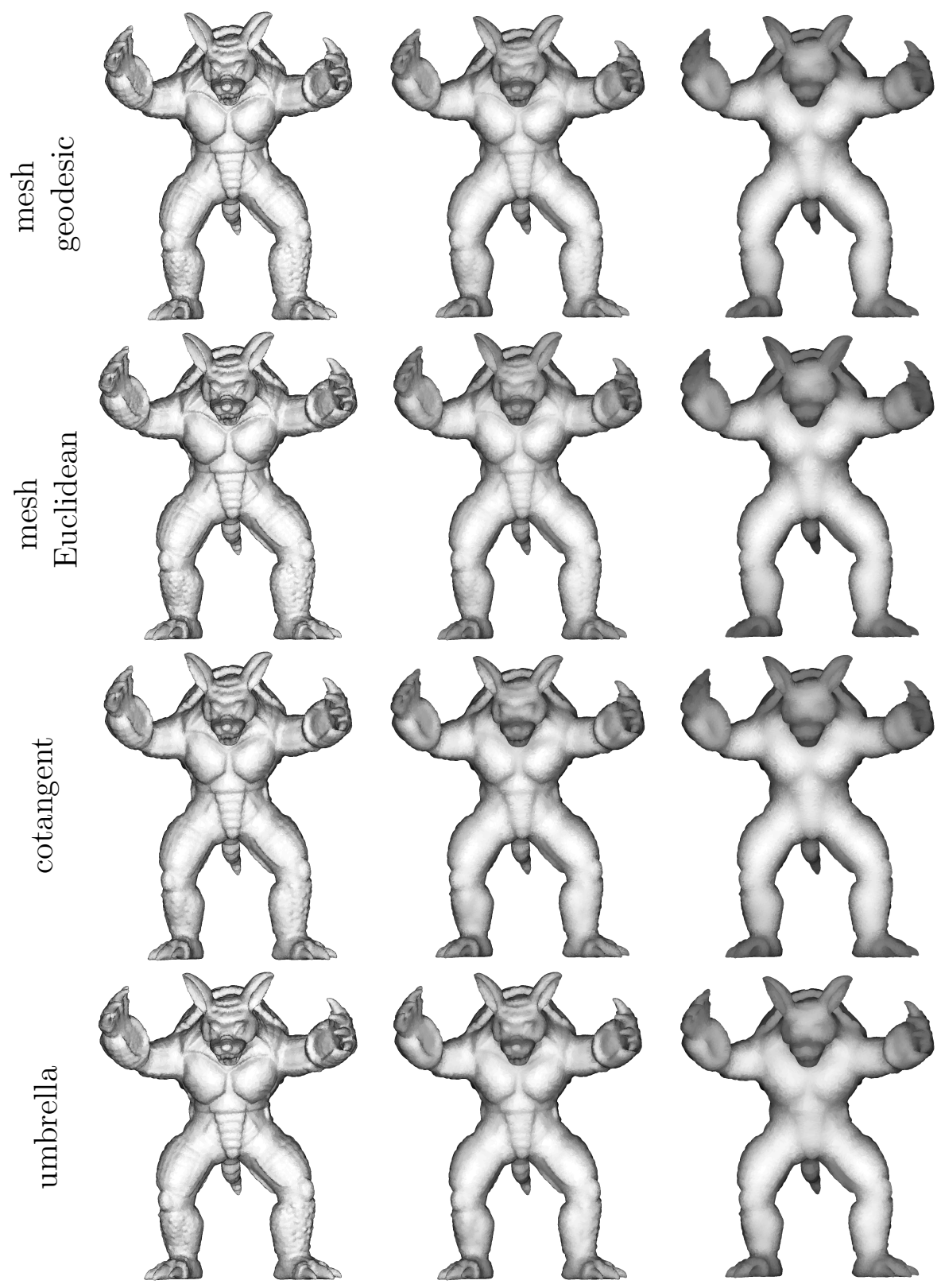

(a) $\sigma=1.50 \bar{e}$

(b) $\sigma=3.35 \bar{e}$

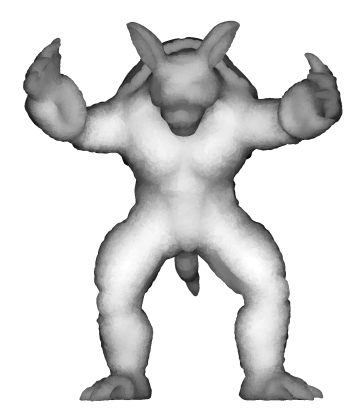

(c) $\sigma=6.71 \bar{e}$

Figure 3.12: Diffusion of Lambertian surface intensity of armadillo model using method DE for various LBOs. 

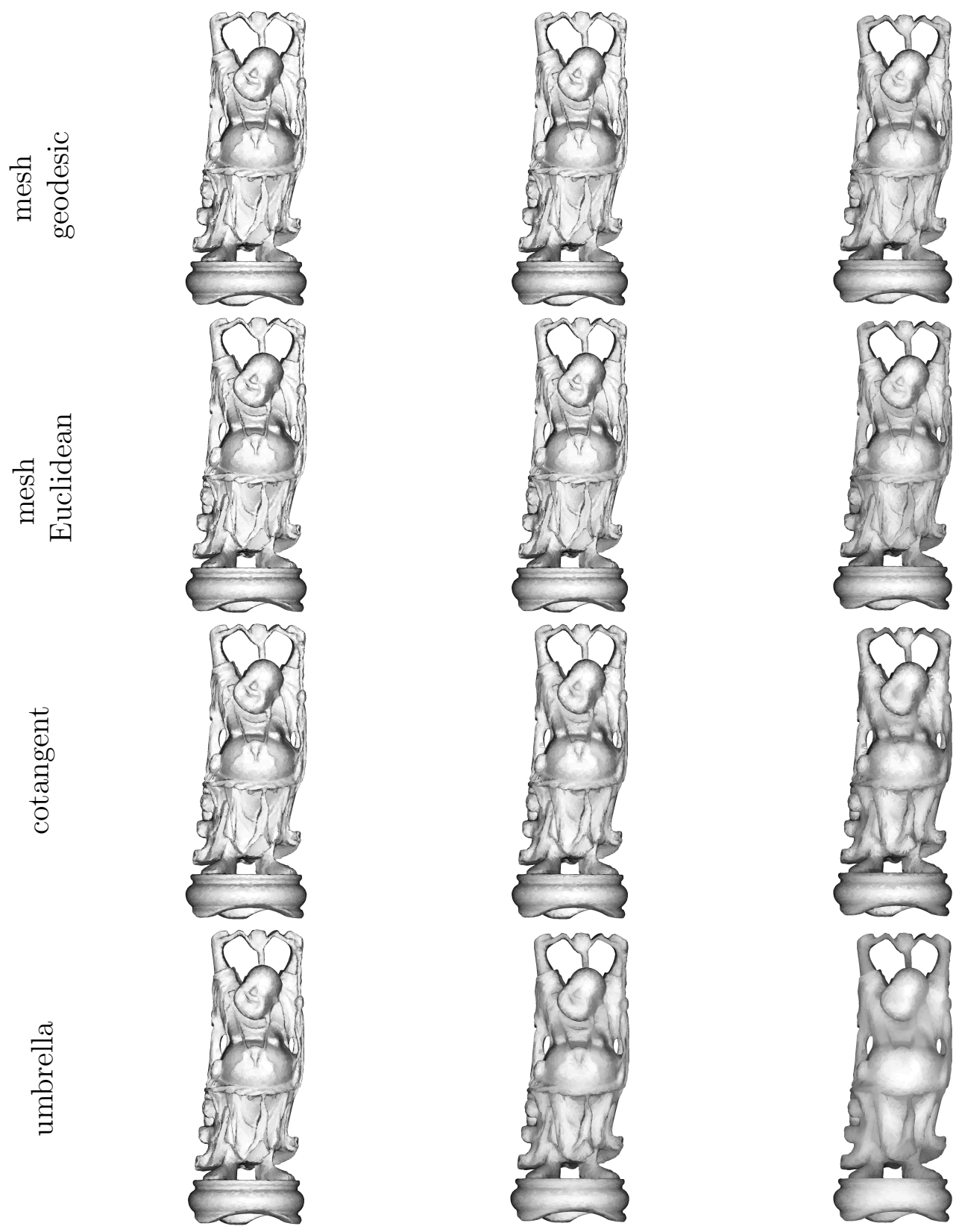

(a) $\sigma=1.50 \bar{e}$

(b) $\sigma=3.35 \bar{e}$

(c) $\sigma=6.71 \bar{e}$

Figure 3.13: Diffusion of Lambertian surface intensity of buddha model using method DE for various LBOs. 

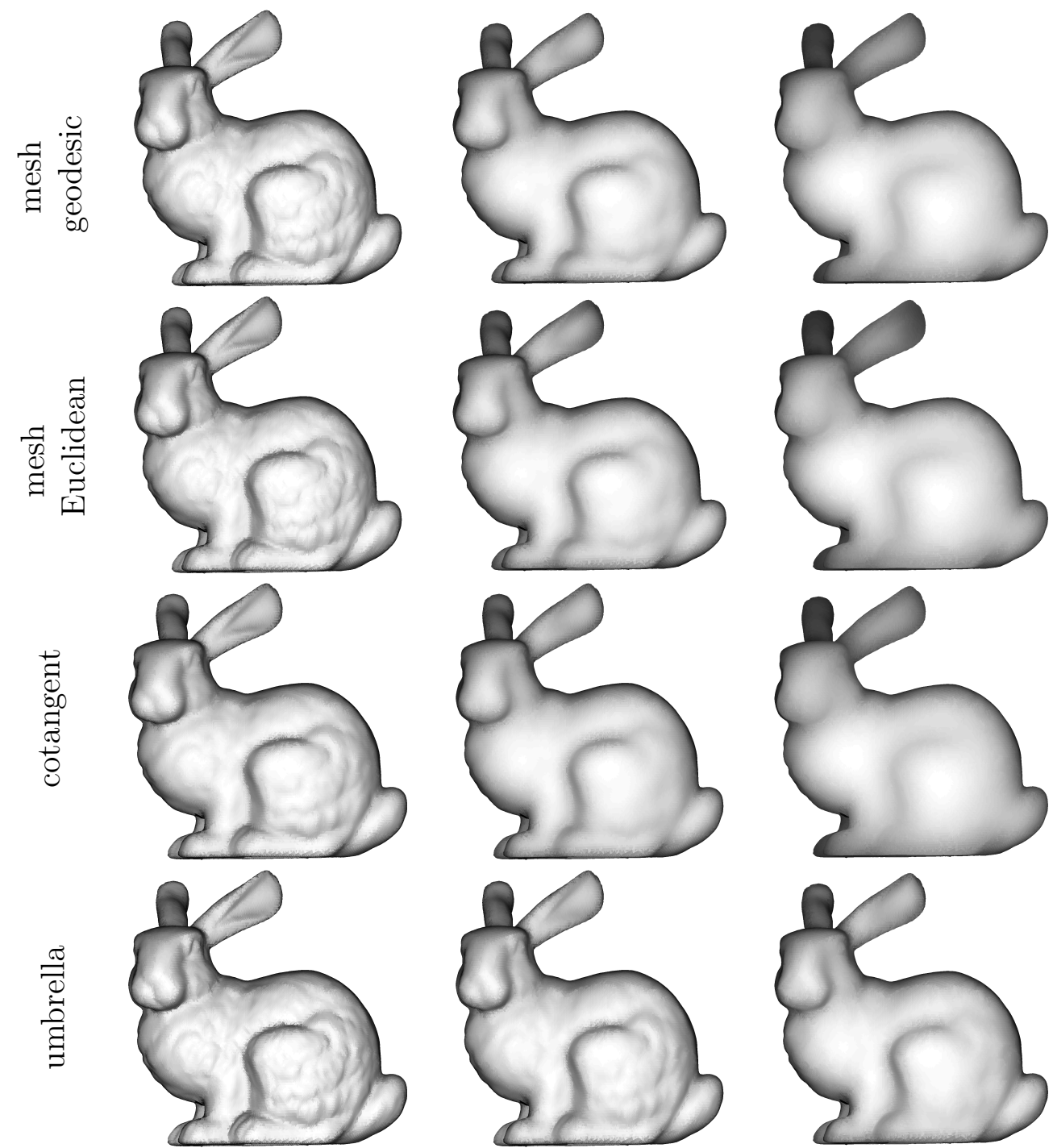

(a) $\sigma=1.50 \bar{e}$

(b) $\sigma=3.35 \bar{e}$

(c) $\sigma=6.71 \bar{e}$

Figure 3.14: Diffusion of Lambertian surface intensity of bunny model using method DE for various LBOs. 

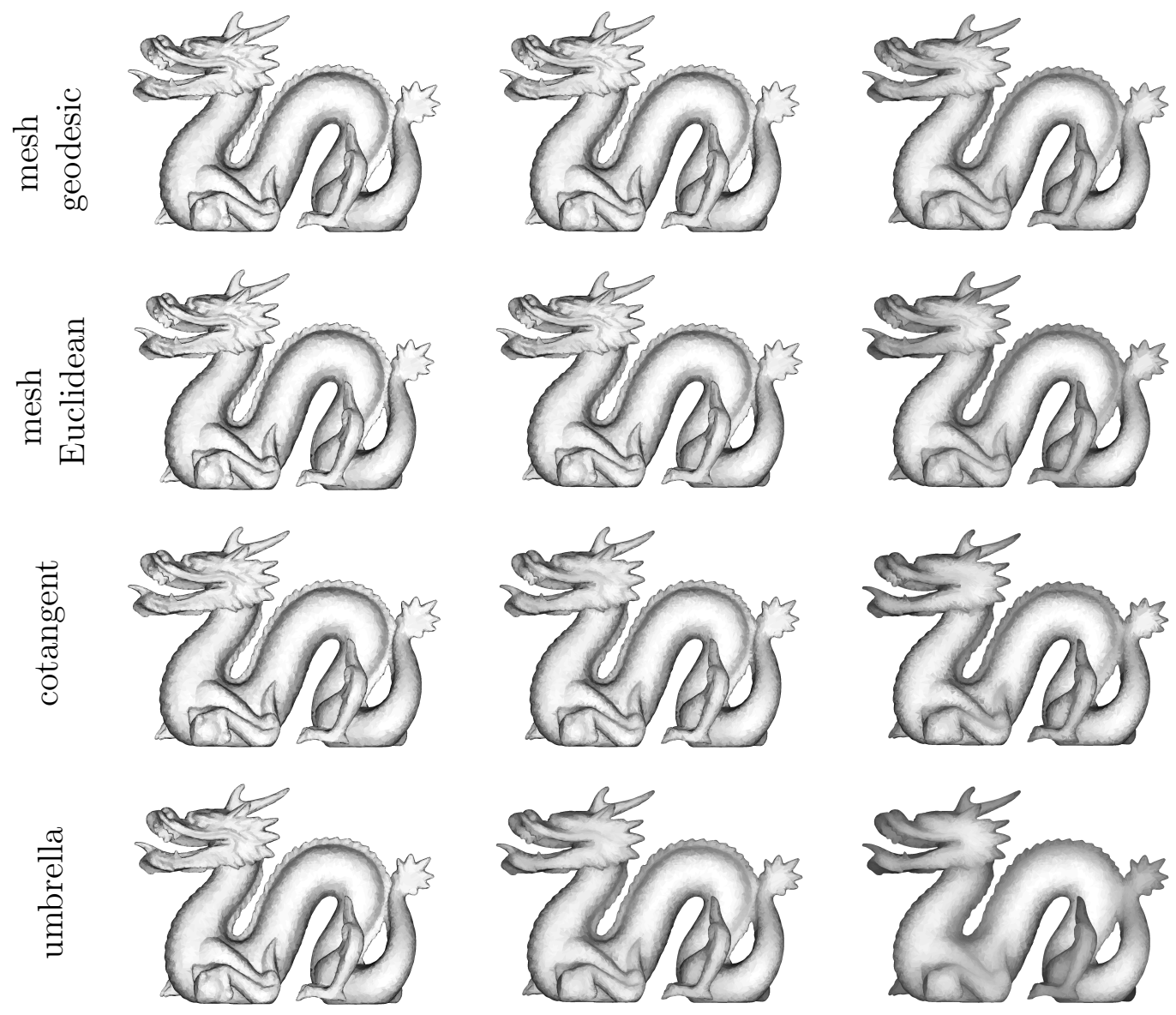

(a) $\sigma=1.50 \bar{e}$

(b) $\sigma=3.35 \bar{e}$

(c) $\sigma=6.71 \bar{e}$

Figure 3.15: Diffusion of Lambertian surface intensity of dragon model using method DE for various LBOs. 

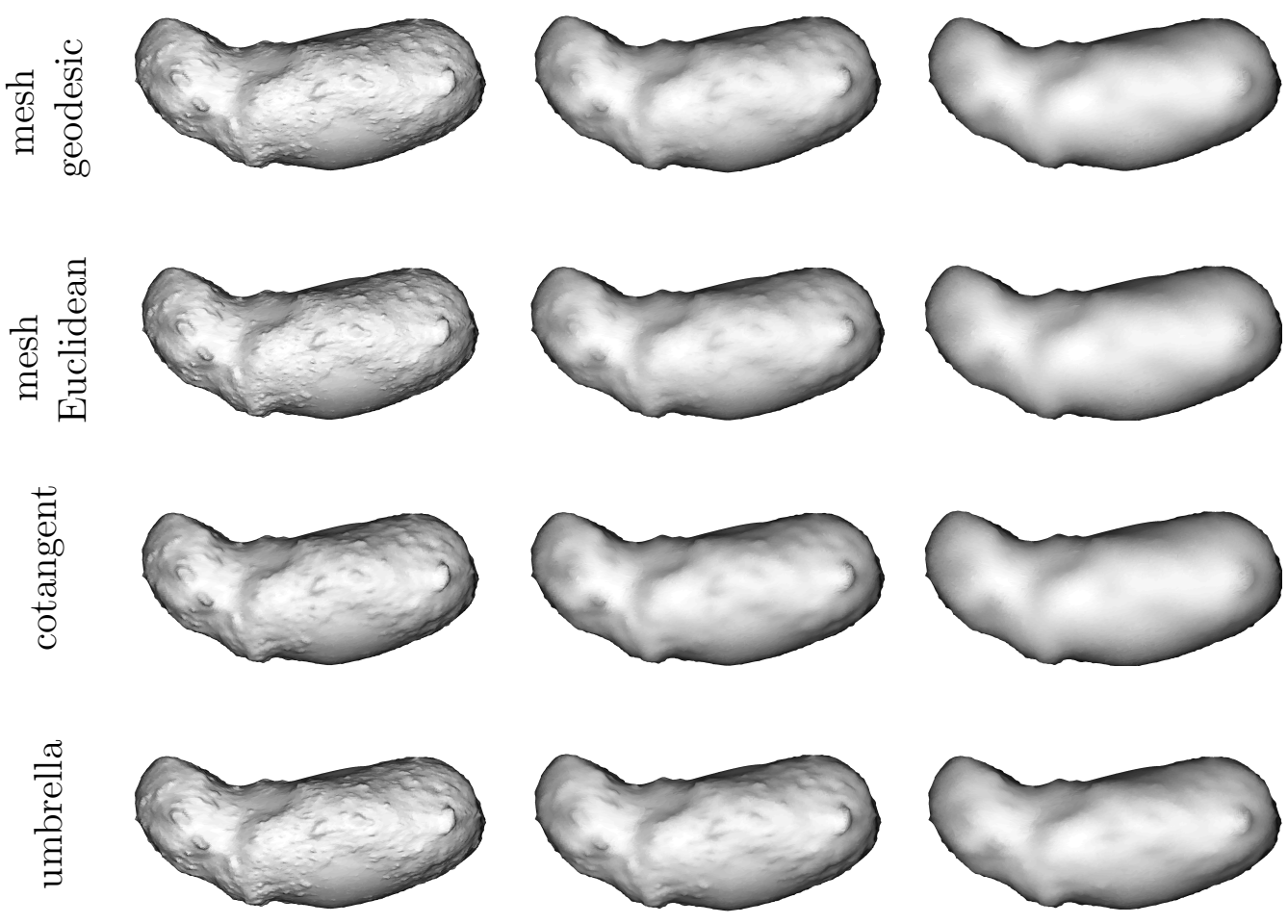
(a) $\sigma=1.50 \bar{e}$
(b) $\sigma=3.35 \bar{e}$
(c) $\sigma=6.71 \bar{e}$

Figure 3.16: Diffusion of Lambertian surface intensity of itokawa model using method DE for various LBOs. 
literature focuses on signal diffusion $[69,67,66,63]$ instead of diffusing mesh vertex locations [63]. The general concept of discrete Gaussian construction are similar in most of these publications. The differences arise when constructing a scale-space, which will be discussed in Chapter 4. This section serves to complete the discussion on diffusion techniques on 3D meshes and to present consistent notation through this document.

\subsubsection{Diffusion with discrete Gaussians}

Recall that the characteristic solution to the diffusion equation is the Gaussian. To implement Eq. 3.1 using discrete Gaussian begin by stacking the signal $\mathcal{U}$ into the vector $\mathbf{u}$. Define the discrete Gaussian in matrix form as

$$
\mathbf{G}_{(i j)}= \begin{cases}\frac{1}{\sigma^{3}(2 \pi)^{3 / 2}} \exp \left(\frac{-\left\|\mathbf{v}_{i}-\mathbf{v}_{j}\right\|^{2}}{2 \sigma^{2}}\right) & \text { if }\left\|\mathbf{v}_{i}-\mathbf{v}_{j}\right\| \leq \rho \sigma \\ 0 & \text { otherwise }\end{cases}
$$

where $\sigma$ is the standard deviation of the Gaussian, $\rho$ is the user-defined scalar that when multiplied by $\sigma$ defines the Gaussian support, and $\|\cdot\|$ is either the geodesic or Euclidean distance metric acting on the vertex locations. We maintain similar default values as Sec. 3.4 (which also is constructed with Gaussian weights) for $\sigma=\bar{e}$ and $\rho=4$. Belkin shows that the Euclidean distance metric is accurate to third order, and thus may replace the geodesic metric for dense meshes [115]. Both distance metrics are analyzed in this work. While the geodesic metric constructs a more accurate Gaussian matrix, the Euclidean metric is computationally less expensive.

The discrete construction of $\mathbf{G}_{(i j)}$ may not satisfy the constraint that 
rows have unit sum, so it is renormalized by row as

$$
\mathbf{G}_{(i j)}=\frac{\mathbf{G}_{(i j)}}{\sum_{j} \mathbf{G}_{(i j)}}
$$

In this way, the total amount of signal is preserved. Fig. 3.17 shows examples of the sparse structure of the discrete Gaussian for explicit surfaces using both the geodesic and Euclidean distance metrics. Parameters chosen for this parameterization are $\sigma=1.6 \bar{e}$ and $\rho=4$. Notice the similarity to the mesh LBO in Fig. 3.7.

Diffusion on explicit surfaces with a Gaussian is implemented as

$$
\mathbf{u}\left(\sigma_{n+1}\right)=\mathbf{G}\left(\varsigma_{n}\right) \mathbf{u}\left(\sigma_{n}\right)
$$

where $\varsigma_{n}$ is the time step such that

$$
\varsigma_{n}^{2}=\sigma_{n+1}^{2}-\sigma_{n}^{2}
$$

\subsubsection{Mesh Diffusion Examples}

Performance evaluations for visual appearances are performed in this section. Our objective is to verify that our method GE is equivalent to that of Gaussian diffusion on 2D images. Testing begins with an impulse on an image, followed by diffusion of a general image, and finally diffusion of a signal on a general 3D surface. Compare examples of impulse diffusion to that of Gaussian diffusion in Fig. 3.4. 


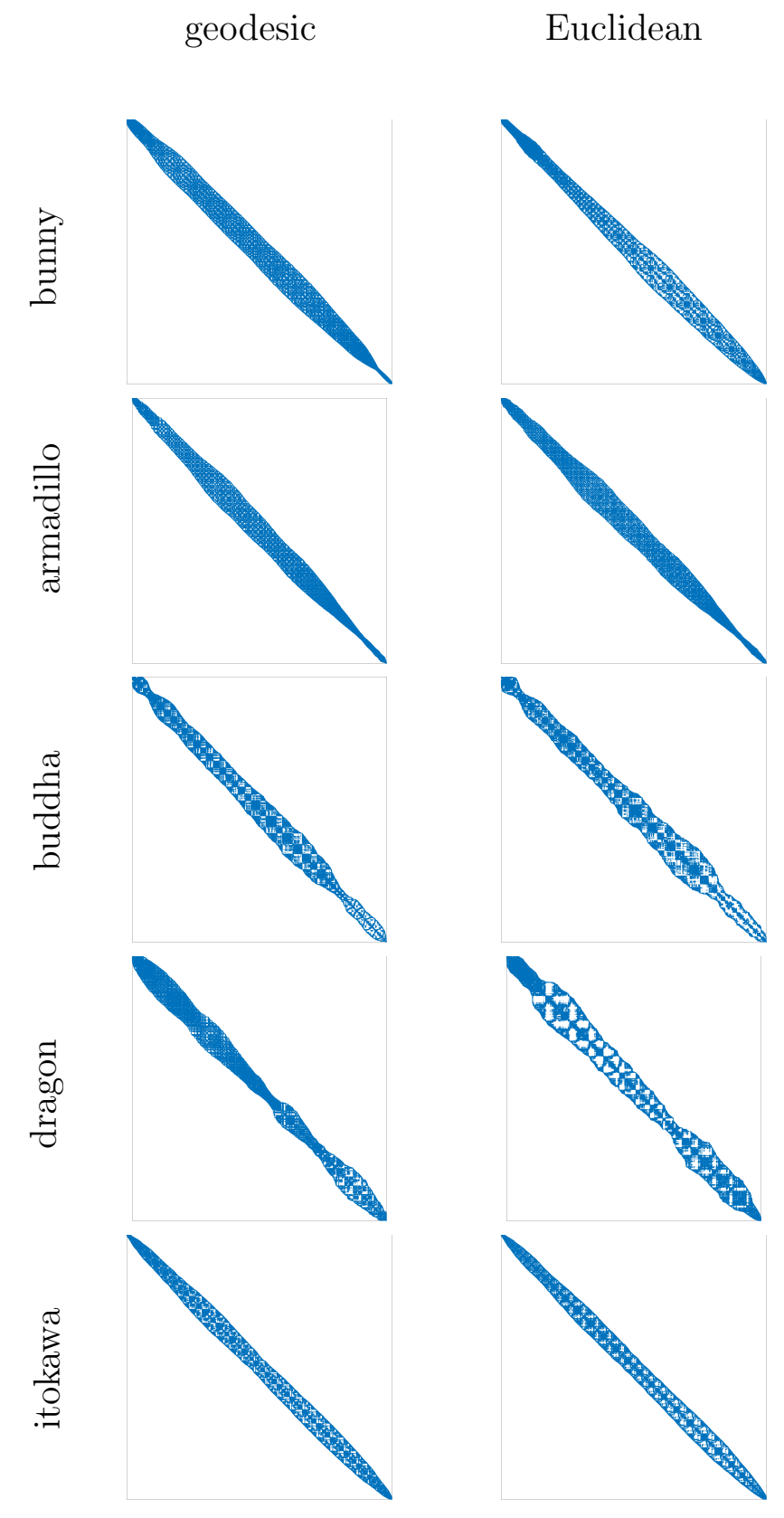

Figure 3.17: Visualization of the explicit Gaussian sparsity for various mesh models. Rows are categorized by model, while columns are categorized by distance metric. 

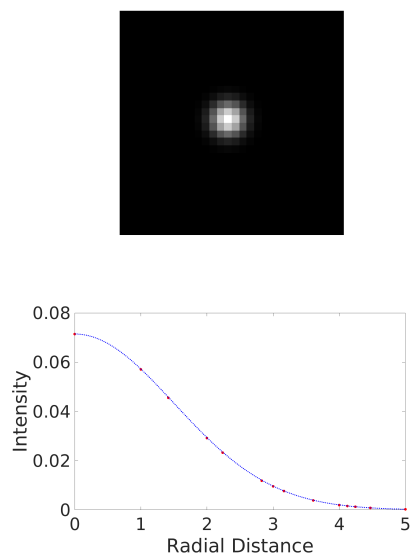

(a) $\sigma_{5}=1.500$
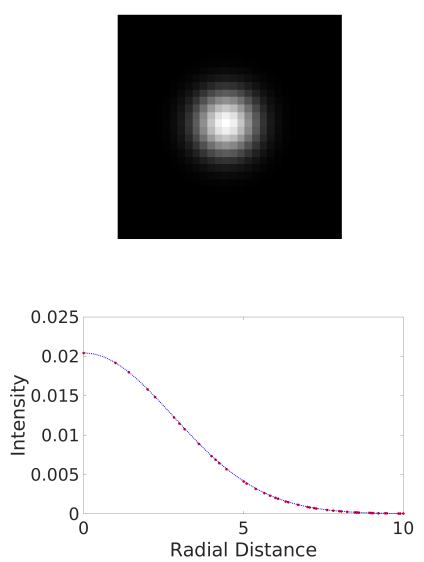

(b) $\sigma_{5}=2.806$
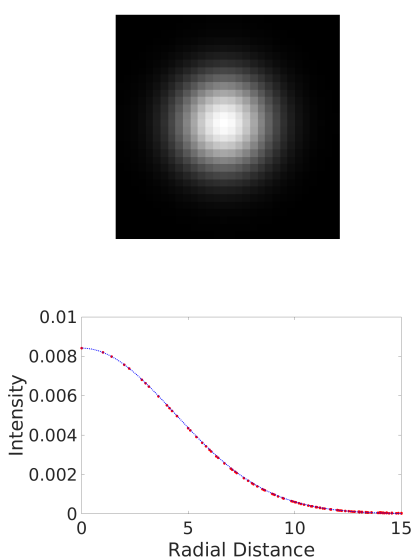

(c) $\sigma_{5}=4.373$

Figure 3.18: Impulse diffusion on an explicit planar mesh using method GE matches extremely well to Fig. 3.4. Red dots are the numeric values of the intensity as a function of radial distance from the center. Blue dotted line is the Gaussian at that scale. Notice the difference in the abscissa limits.

\subsubsection{Diffusion of an Impulse}

A 3D planar mesh is constructed and assigned an impulse signal at the center vertex. Method GE is applied using the settings $\sigma=\bar{e}$, Euclidean distance, and $\rho=4$. Three time steps are shown in Fig. 3.18. Intensities of each image are normalized for visual purposes. The bottom row shows the intensity as a function of radial distance from the center vertex overlaid on a Gaussian with the true standard deviation.

Notice the accuracy of method GE on planar meshes. Since a 3D planar mesh with homogeneously sampled vertex locations, it is nearly equivalent to 
a 2D image. Moreover, since it is planar, the Euclidean and geodesic distances are identical. Therefore, diffusion with method GE on a 3D planar mesh is structurally equivalent to $2 \mathrm{D}$ image diffusion. This equivalence, and the simplicity of constructing $\mathbf{G}$, is what makes method GE attractive to use on general 3D meshes.

\subsubsection{Diffusion of a Monochrome Image}

Diffusion of a planar mesh with a general signal is considered. A planar mesh is constructed with an identical size to the image of Comet $67 \mathrm{P} / \mathrm{CG}$ with

the image signal overlaid directly on the mesh vertices. Diffusion of method GE is compared to 2D image Gaussian diffusion. Parameters for the method GE are $\sigma=\bar{e}$, Euclidean distance, and $\rho=4$. Diffusion results are shown in Fig. 3.19 with 2D image Gaussian diffusion in the top row and method GE in the bottom row.

Following the equivalence statements on the impulse example, this comparison also shows the equivalence of method GE on a planar mesh to 2D image Gaussian diffusion. Showing the similarities of diffusion on a simple mesh such as the plane - allows for the extension of our method GE to general 3D meshes.

\subsubsection{Diffusion of a 3D Model}

We apply diffusion using method GE to five general 3D mesh models. We diffuse a signal constructed by Lambertian reflectance along the surface. 

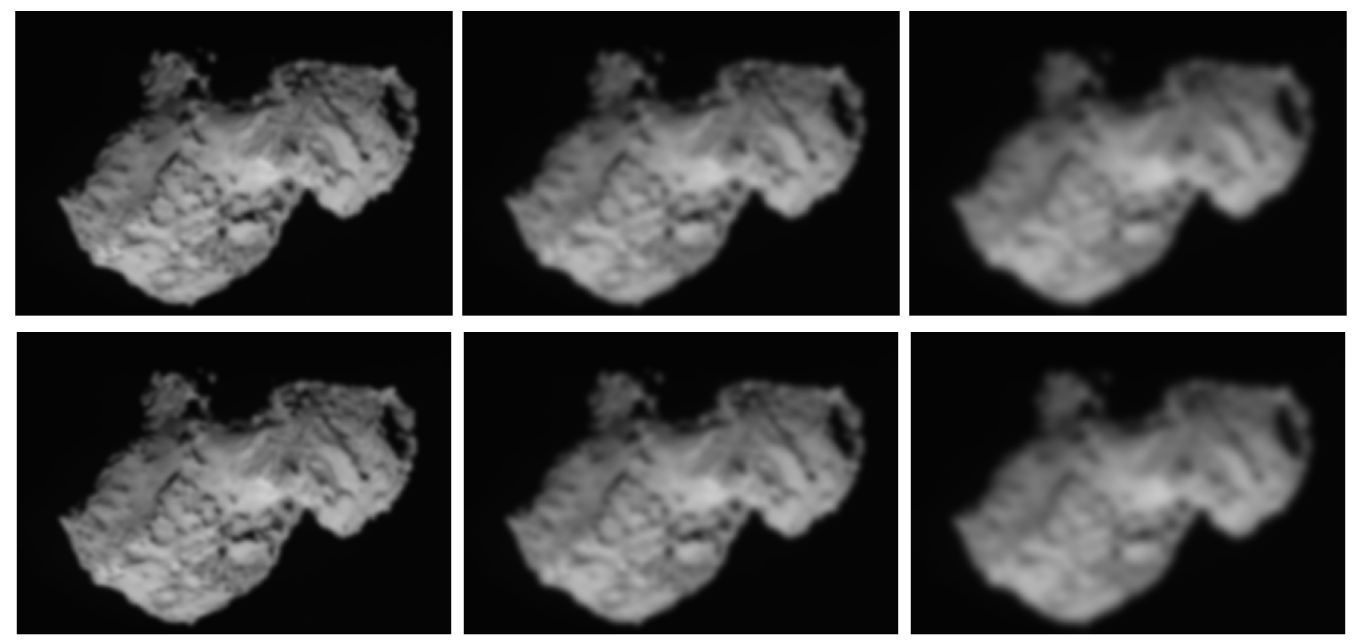

(a) $\sigma_{50}=3.54$

(b) $\sigma_{100}=5.0$

(c) $\sigma_{200}=7.07$

Figure 3.19: Diffusion of a monochrome gray image of Comet 67P/CG. Top row is 2D Gaussian diffusion. Bottom row is method GE. 


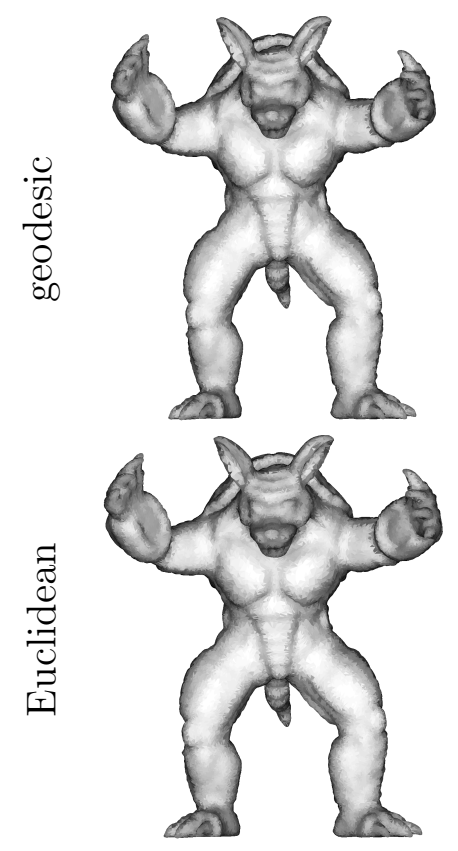

(a) $\sigma=1.47 \bar{e}$
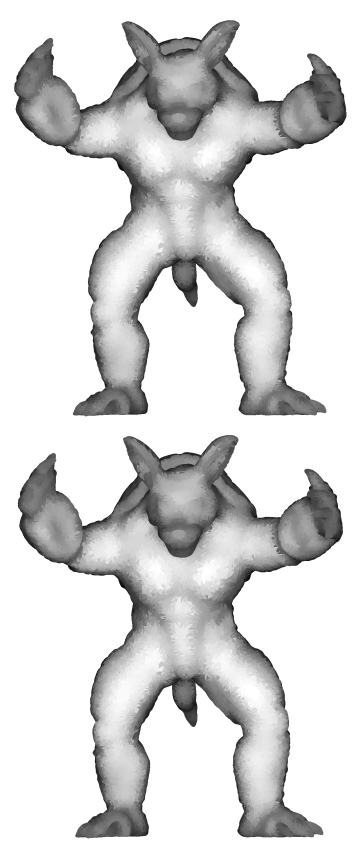

(b) $\sigma=3.02 \bar{e}$
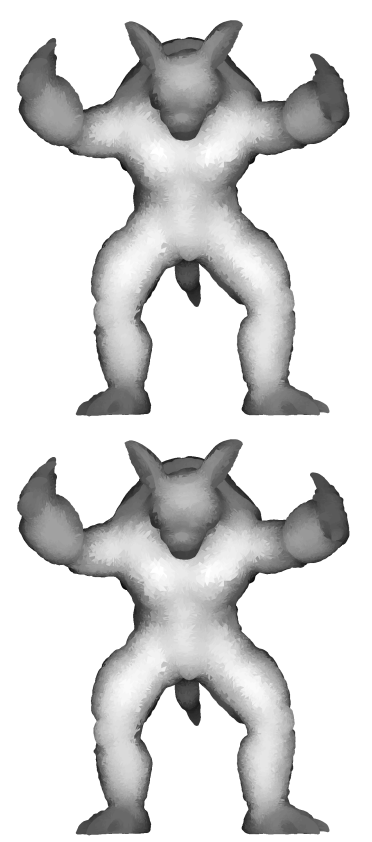

(c) $\sigma=5.93 \bar{e}$

Figure 3.20: Diffusion of Lambertian surface intensity of armadillo model using method GE.

Examples are shown throughout Fig. 3.20, Fig. 3.21, Fig. 3.22, Fig. 3.23, and Fig. 3.24 for three time steps. Results are displayed for both Euclidean and geodesic distance metrics. Diffusion with either distance metric are visually equivalent, showing that Euclidean distances are a good approximation of geodesic distances. 


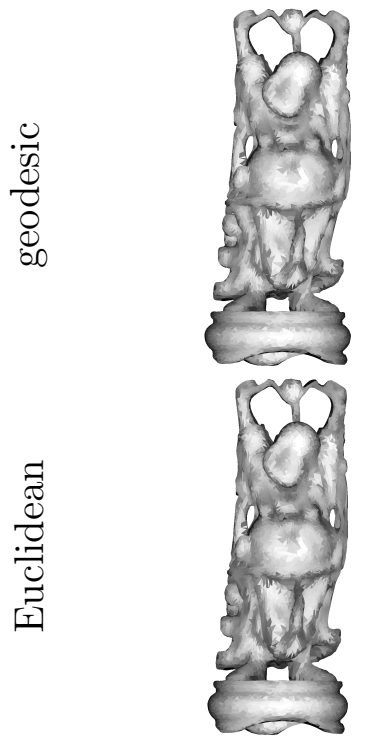

(a) $\sigma=1.47 \bar{e}$
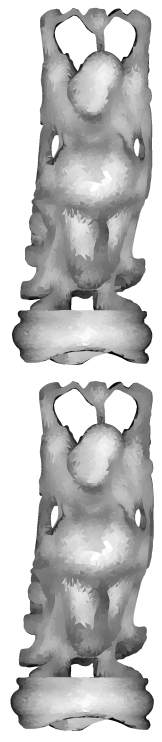

(b) $\sigma=3.02 \bar{e}$
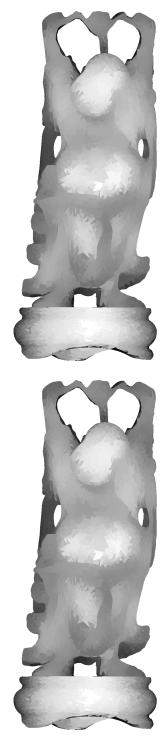

(c) $\sigma=5.93 \bar{e}$

Figure 3.21: Diffusion of Lambertian surface intensity of buddha model using method GE. 

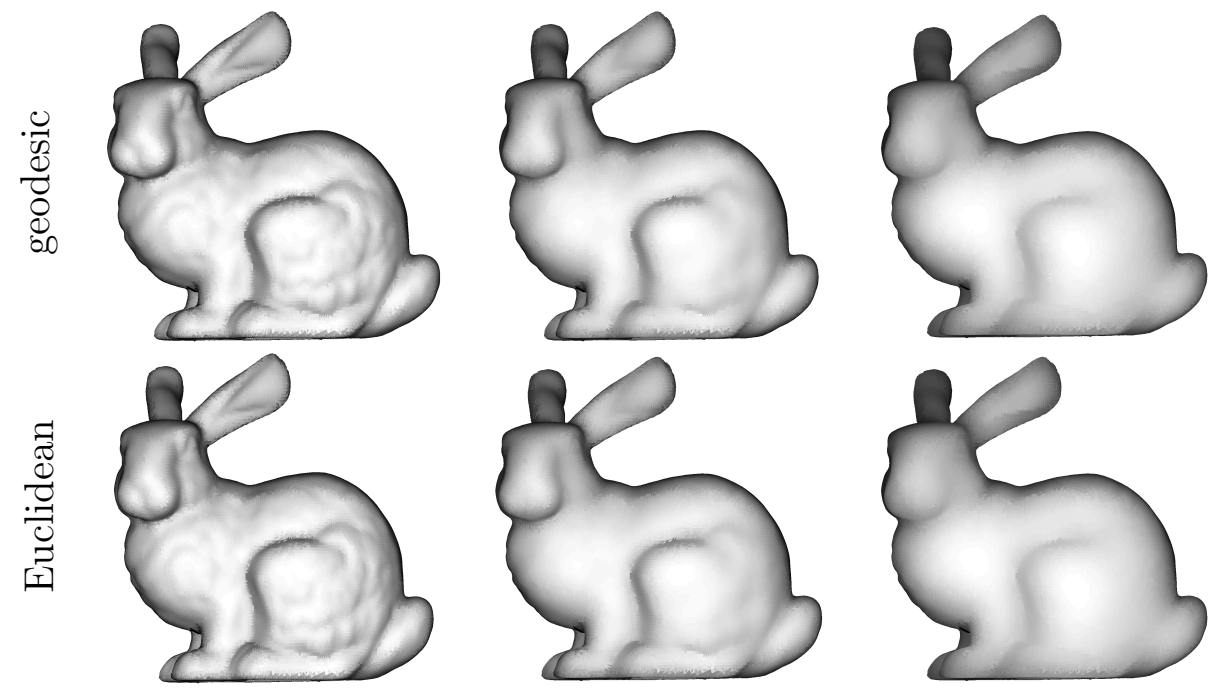

(a) $\sigma=1.47 \bar{e}$

(b) $\sigma=3.02 \bar{e}$

(c) $\sigma=5.93 \bar{e}$

Figure 3.22: Diffusion of Lambertian surface intensity of bunny model using method GE. 

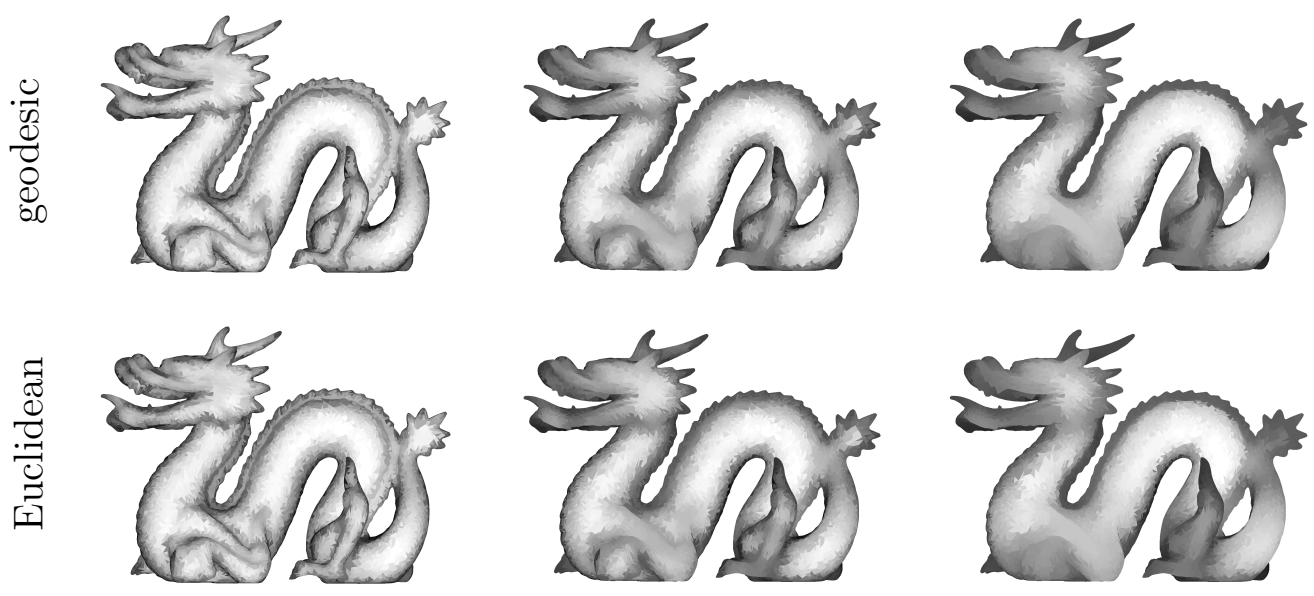
(a) $\sigma=1.47 \bar{e}$
(b) $\sigma=3.02 \bar{e}$
(c) $\sigma=5.93 \bar{e}$

Figure 3.23: Diffusion of Lambertian surface intensity of dragon model using method GE. 

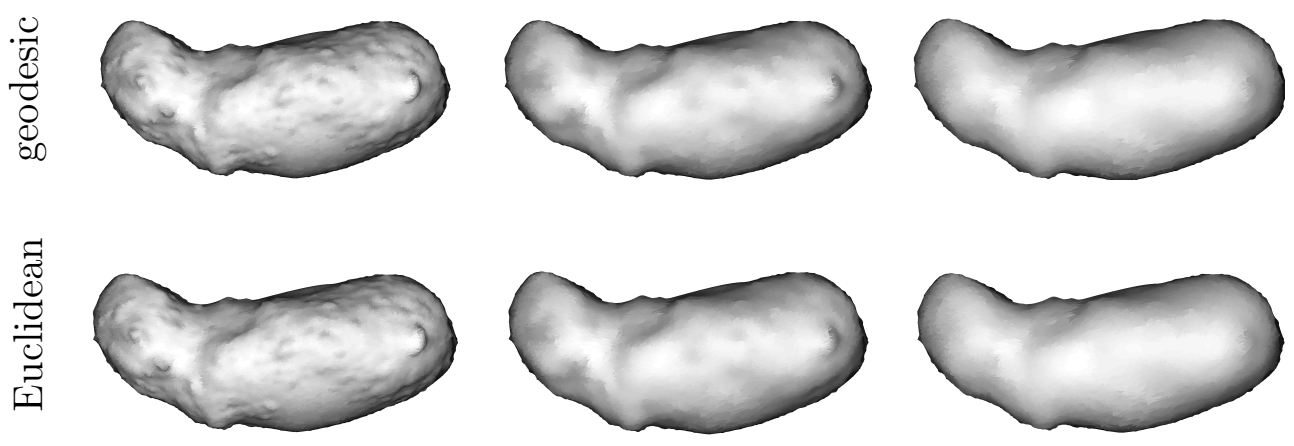

(a) $\sigma=1.47 \bar{e}$

(b) $\sigma=3.02 \bar{e}$

(c) $\sigma=5.93 \bar{e}$

Figure 3.24: Diffusion of Lambertian surface intensity of itokawa model using method GE.

\subsection{Conclusions and Comments}

Implicit surface diffusion methods, while extremely accurate, are too computationally expensive for use in scale-space construction. While the method of DI was investigated in [58], it is not considered a viable approach for keypoint detection in real-time scenarios. Diffusion methods using implicit surfaces, DI and GI, are not extended throughout this manuscript.

Explicit surfaces diffusion methods prove to be faster and sufficiently accurate for scale-space construction. While the method DE requires a numerical method for sparse matrix inversion for every step, the method GE only requires simple matrix multiplication. 


\section{Chapter 4}

\section{Scale-Space Construction}

Scale-space construction is comprised of several individual steps. Step one is an accurate method of diffusion which was discussed in Chapter 3. This chapter proceeds with diffusion on explicit surfaces using both the discretized diffusion with the LBO and Gaussian diffusion. Step two is the selection of particular levels of diffusion to construct a scale-space. It is important that each level of the scale-space is separated by a constant ratio to produce uniform amounts of diffusion between levels. The amount of diffusion is estimated independently of the surface as the scale-parameter. Step three is the application of a keypoint detection operator, for which we use the well-known difference of Gaussians (DoG). Additional optional steps include (1) the refinement of keypoint location in space and scale and (2) the development of a feature descriptor.

Our work makes contributions all three of these steps. First is the identification and classification of diffusion methods with two diffusion operators on two surface representation as presented in Chapter 3. During step two, we notice relationships between the scale-parameter and the diffusion kernel that influence the value of the constant ratio. The amount of diffusion (represented 
by scale) is independent of the LBO, but inherently tied to the Gaussian. These relationship lead to the one-time construction of the diffusion kernel for repeated use in the entire scale-space construction of a particular object. The one-time construction reduces the computationally expensive task of calculating distances on 3D surfaces for kernel construction. For step three, we update to common first-rind neighborhood to a distance-ring neighborhood to more robustly handle non-homogeneously connected surfaces. This work does not refine the location of keypoints in space and scale, nor does is design a new feature descriptor as there are many methods already available in literature.

\subsection{Scale-Parameter Selection and Estimation}

The scale-parameter describes the amount of diffusion that has occurred up to a particular level of the scale-space stack. It is independent of the signal, surface, and surface representation. It is dependent on the selection of $\tau_{n}$ or $\varsigma_{n}$ and the scale grown mechanics of either method DE or GE.

Scale-space analysis requires specific amounts of diffusion to occur between each level of the scale-space stack. The amount of diffusion may be applied either (1) explicitly through the selection of $\tau_{n}$ or $\varsigma_{n}$, or (2) implicitly through the inherent relationship between consecutive convolutions. This section discusses the method for selecting scale-parameters of interest scalespace construction. Relationships between these scale-parameters of interest and consecutive convolutions influence our parameter selections.

Scale-parameters estimation is inherent to diffusion equation and the 
chosen discretized method (e.g. DE or GE), but independent of actually per-

forming diffusion. Estimating the scale-parameter occurs before diffusion to ensure the proper scale-space levels are achieved. This section discusses three methods of estimating the scale-parameter.

\subsubsection{Time Step Selection}

Common convention for constructing a scale-space stack is to separate the scale-parameters by a constant ratio [49]

$$
\sigma_{n+1}=k \sigma_{n}
$$

where $k$ is a positive scalar. The scale-parameters that define the levels of the scale-space stack are defined as

$$
\sigma_{n}=k^{n} \sigma_{0}
$$

where $\sigma_{0}$ is the initial scale factor.

For numerical convenience, SIFT separates the scale-space stack into octaves $O$ each containing an integer number of levels $S$. For each octave, the image is down-sampled by half and the operating scale-parameter resets, while the global scale-parameter still follows Eq. 4.2. Within the levels of an octave, the scale-parameter for the Gaussian is determined by the difference between two consecutive values of Eq. 4.2. In this work, the time steps are selected analogously; however, we maintain a constant mesh throughout the diffusion process without down-sampling. Mesh down-sampling is a computationally 
expensive process that is research interest in itself, and the diffusion kernels (e.g. either LBO or Gaussian) would need to be reevaluated.

\subsubsection{Selecting the Time Step for Method DE}

Our method DE for diffusion on mesh surfaces uses Eq. 3.19, which is a function of $t$ instead of $\sigma$. We must select $\tau_{n}$ in such a way that reproduces the equal ratios in $\sigma$. Recall that the Gaussian variance may be written with either $\sigma$ or $t$ with the relationship

$$
t=\sigma^{2}
$$

Substituting Eq. 4.3 into Eq. 4.1 yields

$$
t_{n+1}=k^{2} t_{n}
$$

which leads to

$$
\tau_{n}=t_{n+1}-t_{n}=k^{2} t_{n}-t_{n}=\left(k^{2}-1\right) t_{n}
$$

such that the accumulated scale at level $n$ becomes

$$
t_{n}=k^{2 n} t_{0}
$$

where $t_{0}$ is the initial scale factor. It is not necessary that the time step ratios be spaced identically as SIFT, so long as $k>1$. These $t_{n}$ values are the desired scale-parameters for analyzing the scale-space stack with the DoG. The discrete time steps are found with Eq. 3.20.

While BDF1 is unconditionally stable for all values of $\tau_{n}$, it is only a first order method, so relatively small steps must be maintained to ensure numerical accuracy. The exponential growth of diffusion time negatively affects 
accuracy when the difference between two consecutive levels becomes large. Additionally, the time steps for numerical integration do not necessarily need to be $\tau_{n}$. Any size steps may be taken for numerical accuracy, so long as the levels corresponding to $t_{n}$ are calculated and retained to construct the scale-space stack.

\subsubsection{Selecting the Time Step for Method GE}

Our method GE for diffusion on mesh surfaces uses Eq. 3.29 with the discrete Gaussian from Eq. 3.27. Since the Gaussian kernel variance may be represented by either $t$ or $\sigma$, both Eq. 4.2 and Eq. 4.6 are equivalent in determining the levels for the scale-space stack.

Recall that for method DE, the scale-parameter $t$ is independent of the discrete LBO. Its implementation technique permits the use of any arbitrary $k>1$ value. Contrastingly for method GE, the Gaussian is dependent on the scale-parameter. Any change in $\varsigma$ requires reevaluating the discrete Gaussian. Since this would be extremely computationally expensive, we decide to hold $\varsigma$ constant and rely on the accumulating affect of Gaussian convolution to reach the levels of the scale-space stack.

Suppose a signal is consecutively convolved $n$ times with a Gaussian of standard deviation $\varsigma$. It is equivalent to a single Gaussian convolution with $\sigma$ if

$$
\sigma=\sqrt{n \varsigma^{2}}
$$

It would be advantageous to select $k$ such that levels of the scale-space stack 
are directly achievable with consecutive Gaussian convolutions and without changing $\varsigma$. Common implementations of SIFT [127] set $k=\sqrt[3]{2}$, but we select $k=\sqrt{2}$. With this selection, the discrete Gaussian of Eq. 3.27 must be calculated only once.

\subsubsection{Selecting the Number of Levels}

The number of levels, $N$, for the scale-space stack is calculated by selecting a desired maximum valued for the scale-parameter

$$
\sigma_{\max }=\eta \bar{e}
$$

where $\eta$ is a scalar. Substituting Eq. 4.6 and Eq. 4.8 into Eq. 4.17 and solving for $N$ yields,

$$
N=\left\lceil\frac{\ln \left((\eta \bar{e})^{2} /\left(2 \alpha t_{0}\right)\right)}{2 \ln k}\right\rceil
$$

where $\lceil\cdot\rceil$ is the ceiling operator.

The scale-parameter $\varsigma$ is held constant for all diffusion steps in method GE, but the levels of the scale-space stack are separated by a constant ratio (from Eq. 4.1). Not all diffusion steps with a constant $\varsigma$ match the scales of the scale-space stack. As the number of levels increase, the number of discrete diffusion steps between levels grows exponentially according to $2^{(n-1)}$. In this scenario, it is advantageous to choose $N$ such that the number of steps between levels remains relatively small and computationally efficient. We set $N=10$ or less depending on the physical object size. 


\subsubsection{Estimation Methods}

We discuss three approaches of scale-parameter estimation. The first is the quadratic fitting method presented in [57], but limits to its implementation exist. The second is the cutoff frequency method which relates the transfer function to the discretized diffusion equation. The third uses the scale inherent to the diffusion equation's characteristic solution — the Gaussian. While all three approaches are applicable to method DE, only the third is applicable to method GE. The discussion concludes with a numerical example.

Scale-parameter estimation for method DE occurs in the frequency domain by taking the Fourier transform of the LBO in Eq. 3.19, resulting in the transfer function [104]

$$
\mathrm{h}_{n}=\left(1-\alpha \tau_{n} \omega^{2}\right)^{-n}
$$

where $\omega^{2}$ is the angular frequency representation of the discrete $\mathrm{LBO}^{1}$ and $n$ is the step of interest. This transfer function does not produce a Gaussian for every time step; however, it does approach Gaussian as $n$ grows large [42]. The objective is to estimate the Gaussian with frequency scale-parameter $\sigma_{\omega(n)}$ that best fits the transfer function at step $n$, and then convert the frequency scale-parameter back to the spatial domain.

Proceed by sampling the transfer function at a series of frequency values, $\omega_{j}$, resulting in the set $\Gamma=\left\{\omega_{j}, \mathrm{~h}\left(\omega_{j}\right)\right\}_{j=0}^{J}$. This is where the quadratic fitting method of [57] and our cutoff method diverge.

\footnotetext{
${ }^{1}$ The Fourier transform of the LBO may also be represented with the ordinary frequency, where $\omega=2 \pi f$
} 


\subsubsection{Quadratic Fitting Method}

The transfer function from Eq. 4.10 may be rewritten as

$$
\mathrm{h}_{n}(\omega)=\prod_{i=0}^{n-1}\left(1+\tau_{i} \omega^{2}\right)^{-1}
$$

where $\alpha=1$ and $n$ is the level of interest dependent on all lower levels $i$ because Eq. 3.19 is iterative. The frequency scale-parameter is estimated for a Gaussian of form

$$
G_{n}\left(\omega, \sigma_{\omega(n)}\right)=\exp \left(-\omega^{2} /\left(2 \sigma_{\omega(n)}^{2}\right)\right)
$$

by fitting it to the transfer function of Eq. 4.11 using least squares, yielding $[57]$

$$
\sigma_{\omega(n)}^{2}=\frac{\sum_{j=0}^{J} \omega_{j}^{4}}{2 \sum_{j=0}^{J} \omega_{j}^{2} \sum_{i=0}^{n-1} \ln \left(1+\tau_{i} \omega_{j}^{2}\right)}
$$

where the derivation may be found in [128]. The scale parameter obtained in Eq. 4.13 depends upon the maximum sampled frequency band of $\Gamma$. Modifying the frequency band yields a different estimate of the scale parameter.

The problem arises because $\ln \left(G_{n}\left(\omega, \sigma_{\omega(n)}\right)\right)$ is always a concave quadratic function, whereas $\ln \left(\mathrm{h}_{n-1}(\omega)\right)$ is only concave quadratic within a range. Another issue arises when determining the sampled frequency range, which [105] suggests to be set as the range of the LBO eigenvalues. The maximum eigenvalue for the umbrella LBO is $\omega_{J}=2$ [105], but may not be readily available for the mesh and cotangent LBOs. We do not use, nor suggest, this method of scale-parameter estimation. 


\subsubsection{Cutoff Method}

The cutoff frequency of the transfer function may be used to estimate the scale-parameter and by leveraging well-known relationships between a Gaussian in the frequency and spatial domains. The transfer function in Eq. 4.10 effectively acts as a low-pass filter and approaches a Gaussian distribution as $n$ grows large. Recall that the cutoff frequency is often taken to be the half-power point $(-3 \mathrm{~dB})$. Interpolating the sampling $\Gamma$ for the $-3 \mathrm{~dB}$ power level approximates the cutoff frequency $\omega_{c}$. For a Gaussian, the cutoff frequency relates to the frequency scale-parameter $\sigma_{\omega}$ as

$$
\sigma_{\omega(n)}=\frac{\omega_{c(n)}}{\sqrt{2 \ln (\sqrt{2})}}
$$

The frequency scale-parameter transforms to the spatial scale-parameter using the inverse Fourier transform. The Gaussian possesses the convenient property that

$$
\sigma^{2}=\frac{1}{\sigma_{\omega}^{2}}
$$

where $\sigma$ has units of length and exists in the spatial domain. Thus, the cutoff method is not influenced by the maximum sampled frequency $\omega_{J}$. The process of sampling the transfer function, finding the cutoff frequency, and approximating the best-fit Gaussian in the frequency domain is shown in Fig. 4.1 for two different time steps $n$. Notice that the transfer function becomes a closer approximation of a Gaussian as $n$ grows large. 


\subsubsection{Gaussian Relationship Method}

Considering the relationship of scale growth for consecutive convolutions provides a direct analytic approach for preferred estimates of the scaleparameter. The scale-parameter is directly estimated in the time domain by recalling the the structure of the Gaussian that solves Eq. 3.1. The Gaussian kernel is

$$
\mathrm{G}\left(\mathbf{x}, t_{n}\right)=\frac{1}{(2 \pi)^{3 / 2}\left(2 \alpha t_{n}\right)^{3 / 2}} \exp \left(\frac{-\|\mathbf{x}\|^{2}}{4 \alpha t_{n}}\right)
$$

where $\mathbf{x}$ is a generic spatial coordinate. The relationship between the diffusion time and the spatial scale-parameter is

$$
\sigma_{n}^{2}=2 \alpha t_{n}
$$

where many authors $[115,1,129,57]$ set $\alpha=1$, though any value is acceptable, as this constant affects the rate of diffusion as much as $t$ does.

Recall that our numerical technique for method DE implements Eq. 3.19 with discrete time steps $\tau_{n}$. The accumulated diffusion time at $t_{n}$ is defined as

$$
t_{n}=\sum_{i=1}^{n} \tau_{i}+t_{0}
$$

where $t_{0}$ is the initial scale of the signal $\mathbf{u}$.

\subsubsection{Numerical Example}

The scale estimation accuracy of the quadratic fitting method is compared to the cutoff method, where natural scale growth is considered truth. Without loss of generality, we set $\alpha=1$ and maintain a constant valued 


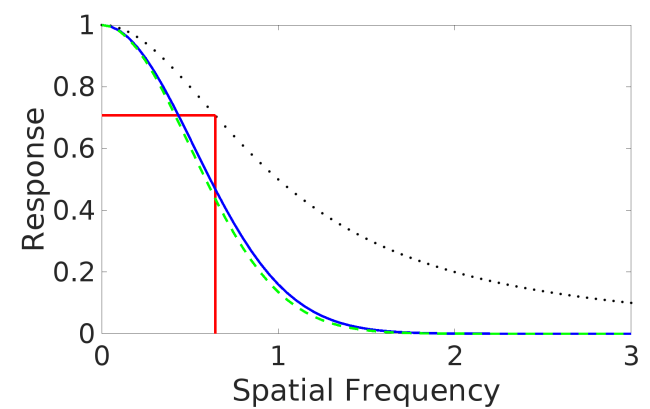

(a) $n=2$

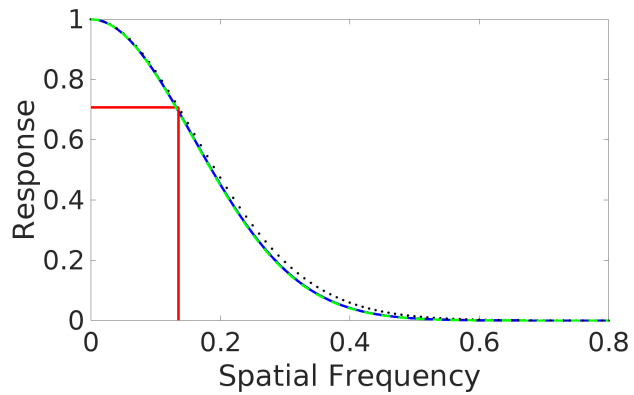

(b) $n=20$

Figure 4.1: The transfer function becomes a closer approximation of a Gaussian as $n$ grows large. Black dots are samples from the actual transfer function in Eq. 4.10. Meeting of the red lines is the cutoff frequency at the half-power point $(-3 \mathrm{~dB})$. Solid blue line is the Gaussian estimated using the cutoff method. Dashed green line is the Fourier transform of Eq. 4.17.

$\tau_{n}=\tau=1 .^{2} \quad$ Also consider three maximum frequency bounds of $\omega_{J}=2$, $\omega_{J}=4$, and $\omega_{J}=5$. The relative errors of the quadratic fitting method and the cutoff method are shown in Fig. 4.1. The cutoff frequency method asymptotically approaches the true scale value as $n$ grows large, and is independent of $\omega_{J}$. The quadratic fitting method displays different error curves depending on the value of $\omega_{J}$.

\subsection{Keypoint Detection, Localization, and Automatic Scale Selection}

After constructing the scale-space stack, keypoints and their support region are found for object description and matching. Among a group of keypoint detectors, the scale-normalized Laplace of Gaussian (LoG), $t \nabla^{2} \mathrm{G}$, pro-

\footnotetext{
${ }^{2}$ In [57] and our work, $\tau_{n}$ is not constant, but this discussion suffices for now.
} 
vides the most stable keypoints [130]. The LoG is computationally expensive, but may be closely approximated with the DoG. Begin with the discretization of Eq. 3.1 in time with a Gaussian as

$$
\frac{\mathrm{G}_{n+1}-\mathrm{G}_{n}}{t_{n+1}-t_{n}} \approx \alpha \nabla_{\mathcal{S}}^{2} \mathrm{G}
$$

where $\tau_{n}=t_{n+1}-t_{n}$ varies with the scale-space level according to Eq. 4.5. Solving for the scale-normalized LoG leads to

$$
\begin{aligned}
\operatorname{DoG}=\mathrm{G}_{n+1}-\mathrm{G}_{n} & \approx\left(t_{n+1}-t_{n}\right) \alpha \nabla_{\mathcal{S}}^{2} \mathrm{G} \\
& \approx\left(k^{2}-1\right) \alpha t_{n} \nabla_{\mathcal{S}}^{2} \mathrm{G}
\end{aligned}
$$

where $\left(k^{2}-1\right) \alpha$ is a constant over all scales, thus not affecting extrema detection. With a signal, it is calculated as

$$
\mathrm{D}(\mathbf{u})=\mathbf{u}_{n+1}-\mathbf{u}_{n}
$$

where D is short for the DoG and also utilized by SIFT [49], MeshDoG [66], GSS [69] and HSS [67].

Vertices whose DoG value achieves an extrema relative to their neighbors in the current level, and the levels immediately below and above, are selected as keypoints. Traditionally, the search neighborhood is the first-ring connected neighborhood $[49,57,129]$, but the next section discusses a different neighborhood construction. The keypoints' scales are automatically selected from the level of the scale-space stack at which the extrema exists. 


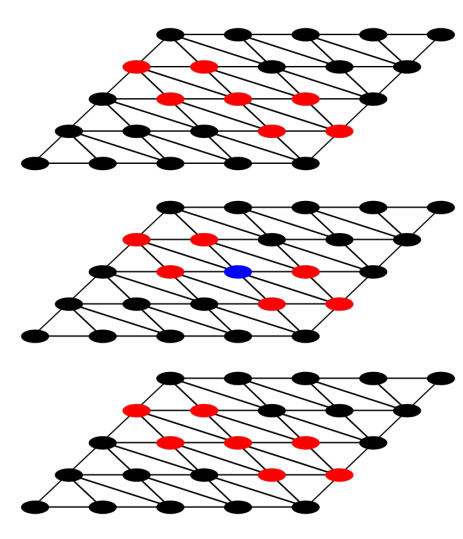

(a) triangular mesh

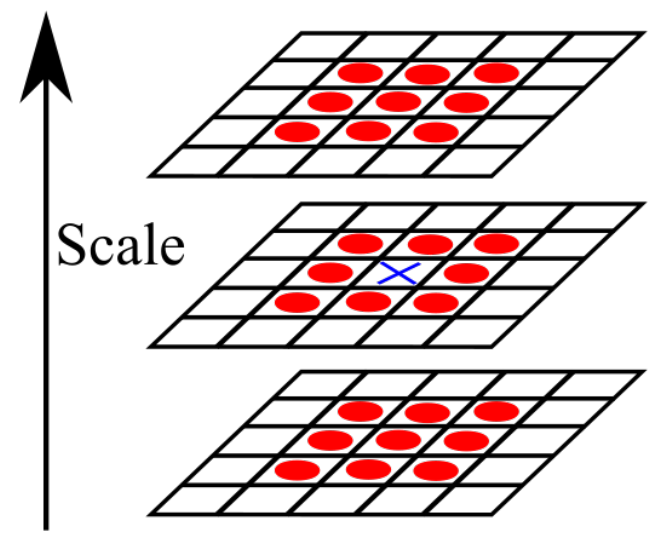

(b) image

Figure 4.2: First-ring neighbor comparison for three consecutive scale-space levels for (a) a triangular mesh and (b) an image. The value of the blue (a) vertex or (b) pixel is compared to the values of the first-ring neighbors in red.

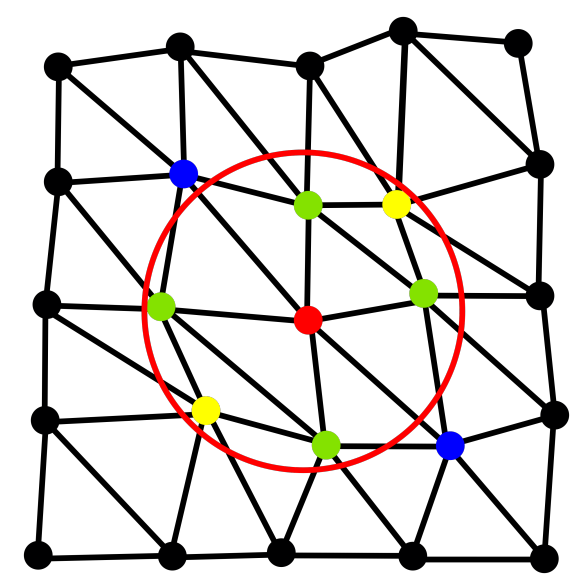

Figure 4.3: Contrasting the distance-ring and first-ring neighborhoods for a vertex (blue) of an inhomogeneous mesh. The first-ring neighbors are the green and yellow vertices. The large red circle shows the boundary for the distance-ring neighbors that includes the green and red vertices, but excludes the yellow vertices. 


\subsubsection{Redefining the Neighborhood}

Consider the neighborhood definition for detecting local extrema. The first-ring connectivity neighborhood is defined by the edges $\mathcal{E}$ of the mesh $\mathcal{M}$. Let's consider this definition for 2D and expand it for higher dimensions.

The first-ring neighborhood of a query pixel in an image is defined as the adjacent eight pixels [49]. The straightforward extension to surfaces defines the first-ring neighborhood as all vertices adjacently connected to the query vertex. Indeed, this is the definition in $[57,129]$ for detecting local extrema, to construct the umbrella and cotangent LBOs, and is denoted here as $\mathcal{N}(\mathbf{v})$. The difference is that images are gridded structures that always have eight connected neighbors, whereas mesh surfaces may have a variable number of neighbors dependent upon the particular model, 3D sensor, or surface reconstruction technique. We propose to define a distance-ring neighborhood that should simplify to the popular first-ring connectivity neighborhoods for specific constraints. A similar definition was proposed in [66].

The distance-ring neighborhood contains all vertices that lie within a geodesic distance (e.g. usually a multiple of $\bar{e}$ or $\bar{e}_{i}$ ) from a queried vertex. This is different from the first-ring neighborhood which is based on connectivity. For example, consider the eight contiguous pixels surrounding a center pixel that form its first-ring neighbors. Each of the eight pixel centers are within a circle

of radius $\sqrt{2}$. Extending to surfaces with a more general average edge length, 
the distance-ring neighborhood is defined as

$$
\mathcal{N}_{d}\left(\mathbf{v}_{i}\right)=\left\{\mathbf{v}_{j} \in \mathcal{V}:\left\|\mathbf{v}_{i}-\mathbf{v}_{j}\right\| \leq \bar{e}_{i} \sqrt{d}\right\}
$$

where $d$ is the dimension ( $d=3$ for this work), and we use $\bar{e}_{i}$ to account for local mesh density variability. This definition uses the geodesic distance metric, but may be approximated with the Euclidean distance. Figure 4.3 shows a graphical representation of the contrast between first-ring and distance-ring neighborhoods. The global average edge length could be used instead of $\bar{e}_{i}$, but this leads to some vertices not having neighbors due to mesh inhomogeneity. This definition of the distance-ring neighborhood does not alter keypoint detection in 2D images or 3D surfaces for specific homogeneous constraints. The list of distance-ring neighbors could be a standard inclusion in object files used for surface diffusion, efficiently constructed by solving the discrete geodesic problem ${ }^{3}$ [131], constructed using an efficient space-partitioning data structure (e.g. k-d trees), or well approximated with the Euclidean distance metric for densely sampled meshes [116].

\subsubsection{Non-Maximum Suppression (NMS)}

Non-maximum suppression (NMS) is a common technique in keypoint detection schemes to reduce the quantity of keypoints based on some characterization of saliency. We apply NMS by defining a minimum scale threshold and by comparing spatial distance and scale similarity.

\footnotetext{
${ }^{3}$ Geodesic package available at https://code.google.com/archive/p/geodesic/
} 
First we compare a keypoint's scale $\sigma_{i}$ to the local average edge length $\bar{e}_{i}$. If $\sigma_{i}<\bar{e}_{i}$, then we remove that keypoint. Next, we sort the remaining keypoints according to their absolute valued DoG response in descending order. Beginning with the first sorted keypoint, which exists at vertex $\mathbf{v}_{i}$ with scale $\sigma_{i}$, we query all other keypoints that lie within a distance threshold $\ell_{d}$. We examine two distance relationships

$$
\begin{aligned}
& \ell_{d}=\sigma_{i} / 2 \\
& \ell_{d}=2 \bar{e}
\end{aligned}
$$

which correspond with distance threshold discussed in Section 4.4.1. If the scale ratio between the given keypoint and the returned keypoint is larger than a threshold, $\sigma_{i / j}>\ell_{r}$ where

$$
\sigma_{i / j}=\frac{\min \left(\sigma_{i}, \sigma_{j}\right)}{\max \left(\sigma_{i}, \sigma_{j}\right)}
$$

then remove the keypoint with the smaller absolute valued DoG response. With this procedure, keypoints that have overlapping support regions with similar scales reduce to a single keypoint. Similar to [49], we set $\ell_{r}=1 / k$.

\subsubsection{NMS for Method DE}

When working the discrete Laplacians, we found that an additional NMS step is necessary. We remove small keypoints according to the empirically found relationships

$$
\begin{cases}\sigma_{i}<\ell_{\sigma_{i}} \bar{e}_{i} & \text { if mesh LBO } \\ \sigma_{i}<\ell_{\sigma_{i}} \sqrt{\bar{e}_{i}} & \text { if cotangent LBO } \\ \sigma_{i}<\ell_{\sigma_{i}} & \text { if umbrella LBO }\end{cases}
$$


where $\ell_{\sigma_{i}}$ is a user selected threshold to define the minimum scale. We set $\ell_{\sigma_{i}}=2$. This method is different than that of [57] which reassigned the scale of small-scaled keypoints. We maintain that these small-scale keypoints are unreliable, susceptible to noise, and often artifacts of the mesh discretization. Thus, they should be removed instead of reassigned.

\subsubsection{NMS for Method GE}

A similar step is also applied when working with a discrete Gaussian. We remove small keypoints if the selected scale is less than the local average edge length. The relationship is

$$
\sigma_{i}<\ell_{\sigma_{i}} \bar{e}_{i}
$$

where $\ell_{\sigma_{i}}$ is a user selected threshold to define the minimum scale. We set $\ell_{\sigma_{i}}=1$.

\subsection{Keypoint Detection Examples}

It is instructive to qualitatively compare the performance of Gaussian scale-space on an image using SIFT with that of our methods DE and GE. These comparisons will show whether our methods DE and GE are comparable to Gaussian scale-space on an image. Test images are of the Voorhees Computing Center ${ }^{4}$ and of Woodburn Hall ${ }^{5}$. To use our methods on an image,

\footnotetext{
${ }^{4}$ Located on the RPI campus

${ }^{5}$ Located on the WVU campus
} 
a planar mesh is constructed such that each vertex corresponds to an image pixel.

After demonstrating the comparability of our methods to 2D image Gaussian scale-space, we apply the methods to general 3D models. We diffuse a signal of mean curvature along the surface of the general 3D models using both methods DE and GE.

\subsubsection{Planar Mesh Example using DE}

Planar meshes with an overlaid signal of a general image are diffused using BDF1 to compare the three LBOs. Other parameters are $\alpha=1, k=\sqrt{2}$, and for $N=10$. The NMS routine from Section 4.2.2 is applied to the SIFT diffusion and the planar mesh diffusion alike. The results are shown in Fig. 4.4

Notice that the keypoints from the mesh and cotangent LBOs most closely resemble one another. Since the planar mesh is homogeneous, and both the mesh and cotangent LBOs incorporate the vertex area into their weighting schemes. Together, they are similar to most of the SIFT keypoints. The differences may be explained by the SIFT procedure of removing keypoints based on their corner response and absolute DoG value, as well as that the method in our work is a numerical approximation to Gaussian diffusion. Keypoints

found with the umbrella LBO are similar in nature to those of SIFT, mesh and cotangent LBOs, but usually at different scales. Additionally, many more 


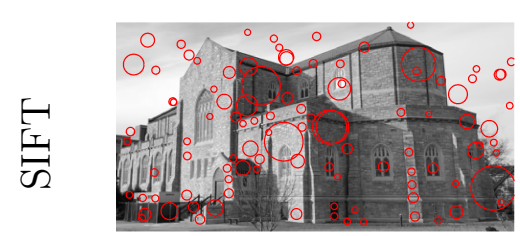

(a) $m_{\mathcal{K}}=116$

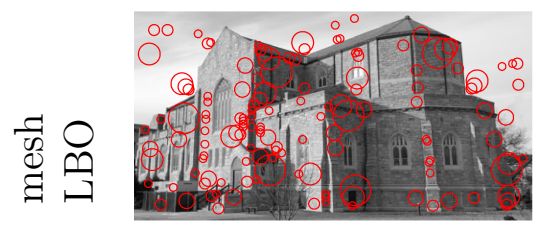

(b) $m_{\mathcal{K}}=138$

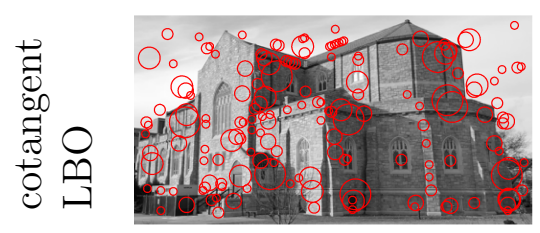

(c) $m_{\mathcal{K}}=162$

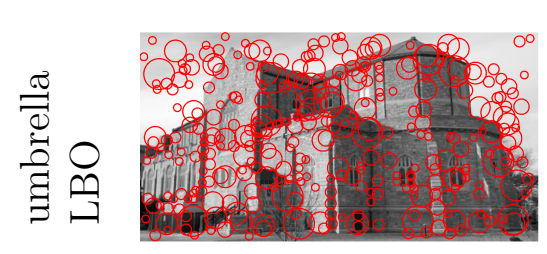

(d) $m_{\mathcal{K}}=328$

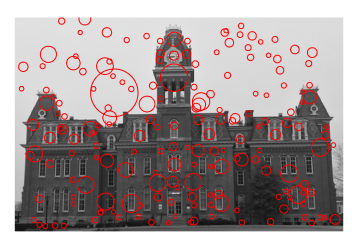

(e) $m_{\mathcal{K}}=217$

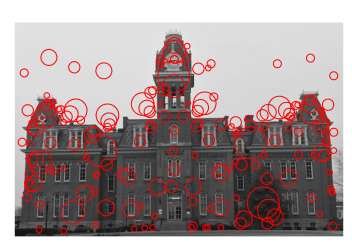

(f) $m_{\mathcal{K}}=176$

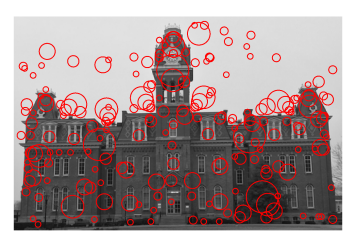

(g) $m_{\mathcal{K}}=200$

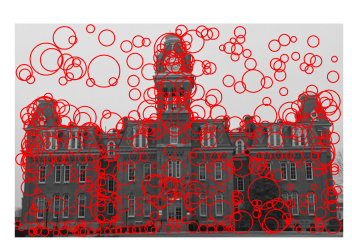

(h) $m_{\mathcal{K}}=428$
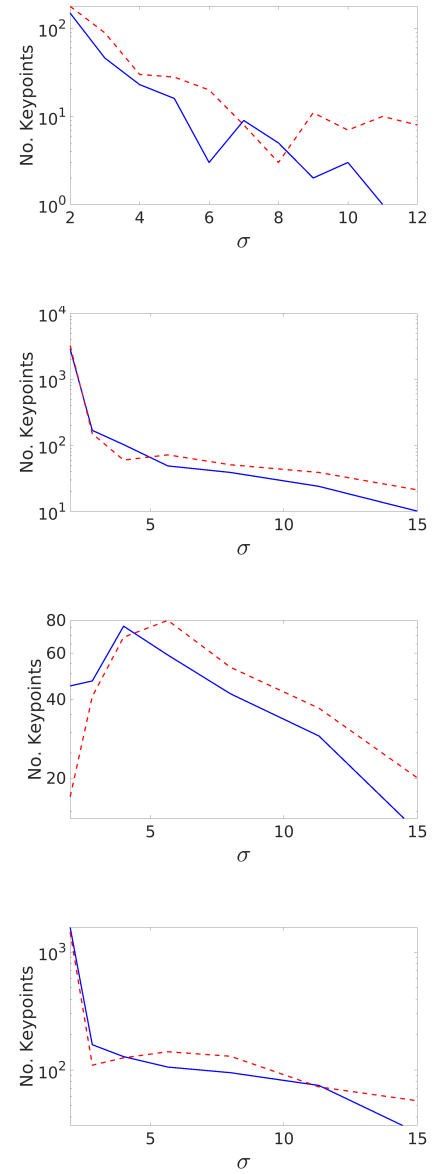

Figure 4.4: Comparing keypoints of image diffusion with SIFT to that of planar mesh diffusion with the LBOs for two images: Voorhees Computing Center and Woodburn Hall. Number of keypoints found are shown in each sub-caption by $m_{\mathcal{K}}$. The right column shows that an abundance of keypoints are found at small scales for all diffusion methods before NMS is applied. Colors correspond to images as Voorhees (solid blue) and Woodburn (dotted red). 


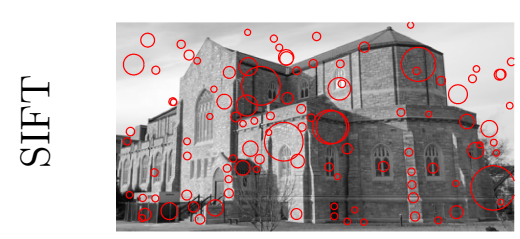

(a) $m_{\mathcal{K}}=116$

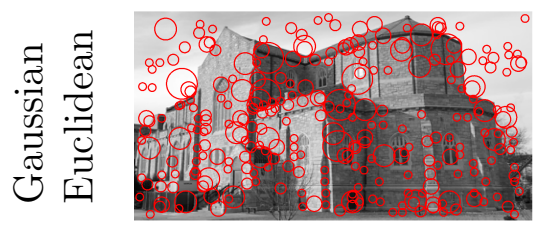

(d) $m_{\mathcal{K}}=281$

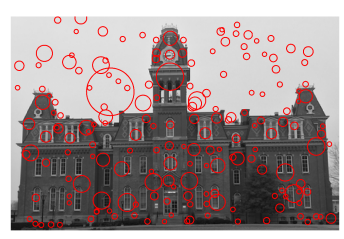

(e) $m_{\mathcal{K}}=217$

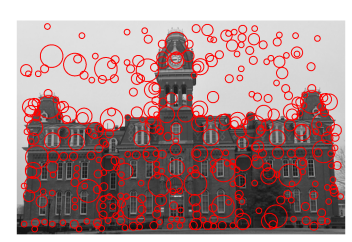

(h) $m_{\mathcal{K}}=371$
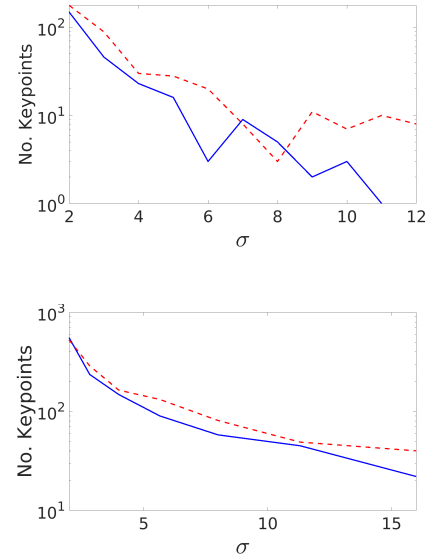

15

Figure 4.5: Comparing keypoints of image diffusion with SIFT to that of planar mesh diffusion with method GE for two images: Voorhees Computing Center and Woodburn Hall. Number of keypoints found are shown in each sub-caption by $m_{\mathcal{K}}$. The right column shows that an abundance of keypoints are found at small scales for all diffusion methods before NMS is applied. Colors correspond to images as Voorhees (solid blue) and Woodburn (dotted red).

keypoints are found with the umbrella LBO. The nominal SIFT method ${ }^{6}$ [49] found many keypoints at low scales that were removed with our NMS strategy.

\subsubsection{Planar Mesh Example using GE}

Diffusion of the general image signal overlaid on a planar mesh is performed using method GE. Since Euclidean and geodesic distances are equivalent on planar meshes, we only compare the Euclidean metric to SIFT. Other parameters are $\rho=3, \sigma=0.5$, and $k=\sqrt{2}$. Results are shown in Fig. 4.5.

\footnotetext{
${ }^{6}$ SIFT implementation is from http://www.vlfeat.org/
} 
Notice that many of the keypoints found with method DE are identical to SIFT, especially around the windows and clock tower. However, method GE finds many more keypoints that SIFT because the SIFT NMS routine differs from ours.

\subsubsection{General Mesh Example using DE}

Signal diffusion is performed using method DE for each of the LBOs on the five models from Fig. 4.8. Common parameter settings are $t_{0}=\bar{e} / 4$, $k=1.2, \alpha=1$, for $N=11$ levels. For the mesh LBO we set $\rho=4$ and use geodesic distances. Keypoints are shown in Fig. 4.6.

These keypoints are found in the scale-space when the signal is mean curvature. Notice the similarities among the keypoints found by each LBO. For the bunny, the mesh and cotangent LBOs find similar keypoints on the back, chest and ears. The umbrella LBO finds many more keypoints on the bunny than the other LBOs. For the armadillo, the mesh and cotangent LBOs find similar keypoints on the chest, legs, and tail. The mesh LBO finds many keypoints round the facial features. For the dragon, the mesh and umbrella LBOs find similar keypoints along the spine at each peak.

\subsubsection{General Mesh Example using GE}

Signal diffusion is performed for method GE using each of the distance metrics for the five models shown in Fig. 4.8. Common parameters are $k=\sqrt{2}$, $\sigma=0.8 \bar{e}, \alpha=1 / 2, N=11$, and $\rho=4$. Keypoints are shown in Fig. 4.7 for a 


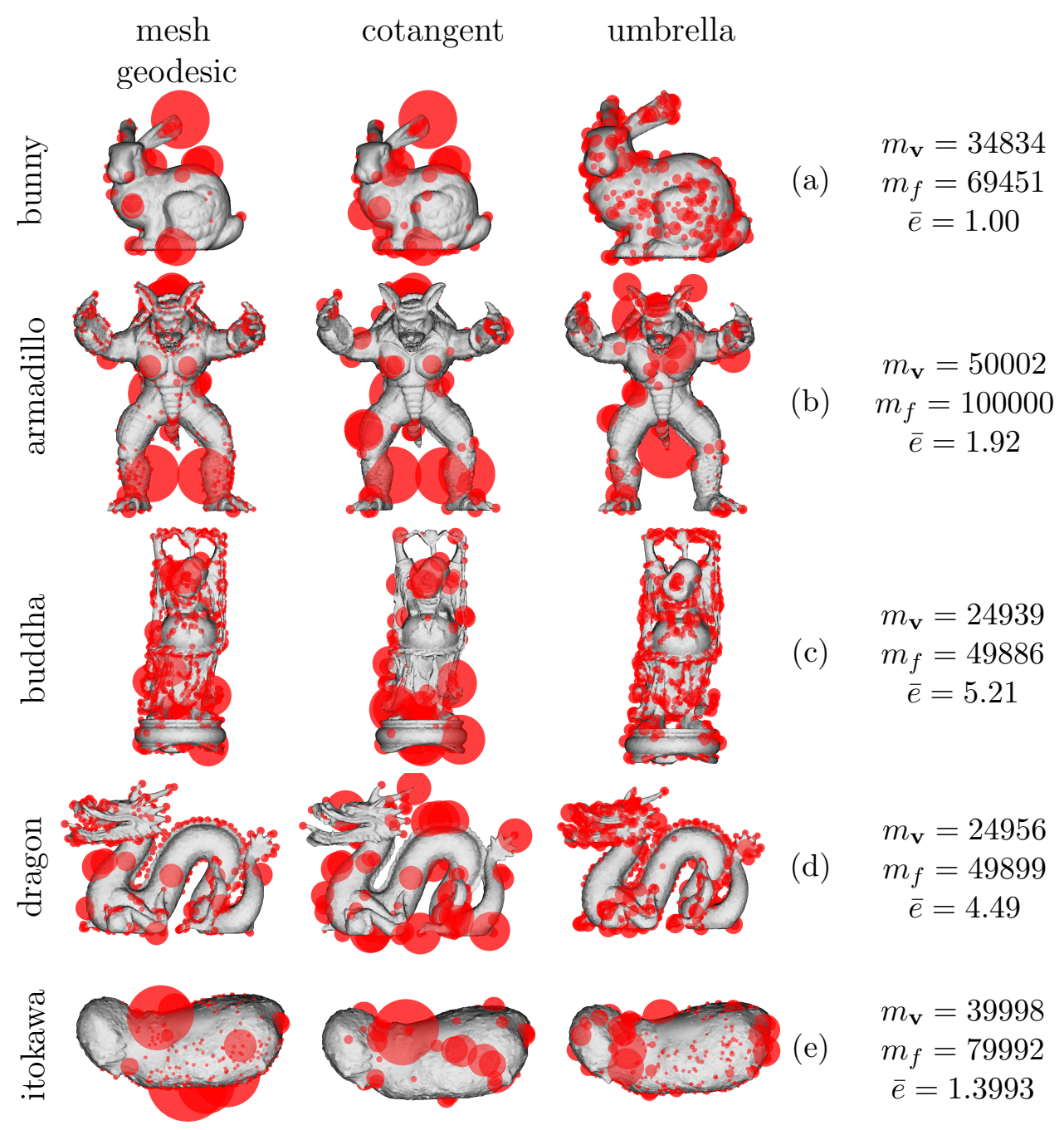

Figure 4.6: Models from the Standford scanning repository and the asteroid Itokawa with keypoints using each of the LBOs. 
signal of mean curvature.

Notice the similar keypoints between the two distance metrics for each model. There are many common keypoints found using method GE as with method DE, such as the armadillo chest and bunny nose. For the dragon though, method GE identifies keypoints at the base of the spine whereas method DE found keypoints on the spine edge. Overall, there are far fewer keypoints for all models using method GE as compared to method DE.

\subsection{Numerical Validation of Keypoint Detection}

The repeatability of keypoint detection and localization using methods DE and GE are evaluated through a number of numerical tests. These experiments diffuse a signal of mean curvature, but any signal is acceptable. For method DE, natural scale selection from Section 4.1.2.3 is used for the scaleparameter estimation. DoG of Eq. 4.21 detects keypoints; however, selection of the neighborhood definitions depends on the selected LBO. The distance-ring neighborhood is selected for the mesh LBO, whereas, the first-ring neighborhood is chosen for the umbrella and cotangent LBOs. While the umbrella LBO has been used before for signal diffusion on 3D meshes [57], we have not found any literature using either the mesh or cotangent LBOs. For method GE, the distance-ring neighborhood is selected for keypoint detection.

Two experiments use Monte Carlo simulations by adding noise to the (1) mean curvature signal or (2) vertex locations. Four models from the Standford Scanning Repository and a model of the asteroid Itokawa [126] are used. 

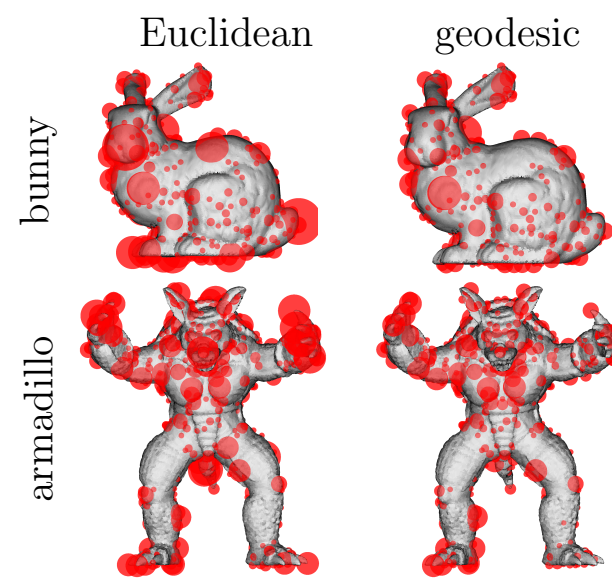

$m_{\mathbf{v}}=34834$

(a) $\quad m_{f}=69451$

$$
\bar{e}=1.00
$$

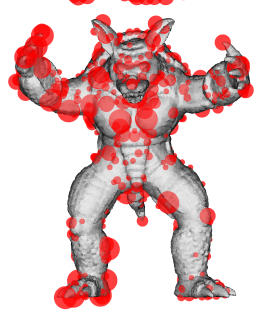
$m_{\mathbf{v}}=50002$
(b) $\quad m_{f}=100000$
$\bar{e}=1.92$
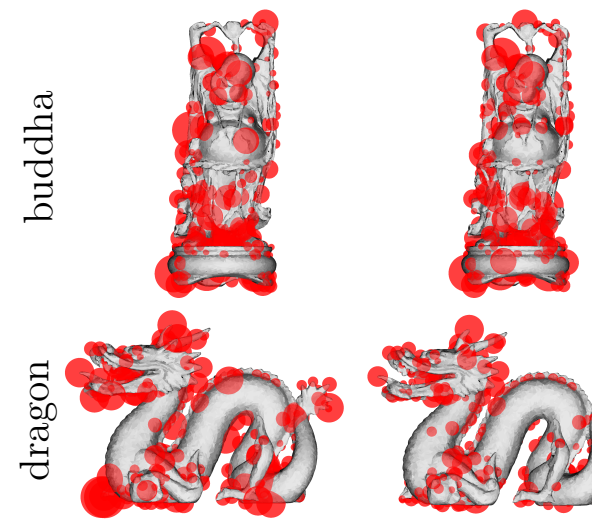

$m_{\mathbf{v}}=24939$

(c) $\quad m_{f}=49886$

$\bar{e}=5.21$
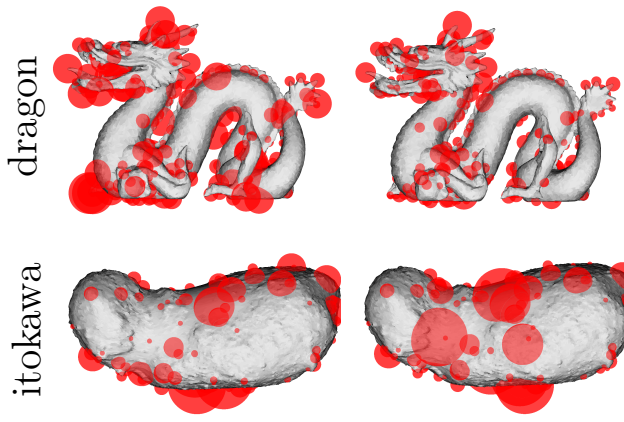
$m_{\mathrm{v}}=24956$
$m_{f}=49899$
$\bar{e}=4.49$

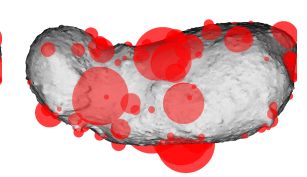

$$
\begin{gathered}
m_{\mathbf{v}}=39998 \\
m_{f}=79992 \\
\bar{e}=1.3993
\end{gathered}
$$

Figure 4.7: Models from the Standford scanning repository and the asteroid Itokawa with keypoints using method GE with both distance metrics. 


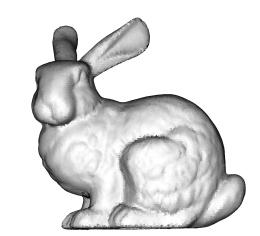

$m_{\mathbf{v}}=34834$

(a) $m_{f}=69451$

$\bar{e}=1.00$

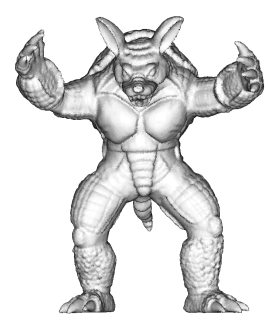

$m_{\mathbf{v}}=50002$

(b) $m_{f}=100000$

$\bar{e}=1.92$

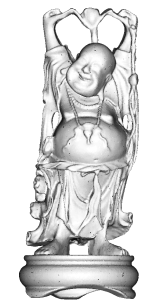

$m_{\mathbf{v}}=24939$

(c) $m_{f}=49886$

$\bar{e}=5.21$

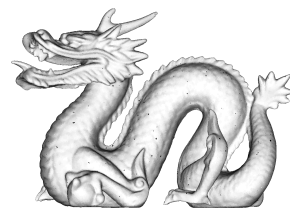

(d) $\begin{aligned} m_{\mathbf{v}} & =24956 \\ m_{f} & =49899 \\ \bar{e} & =4.49\end{aligned}$

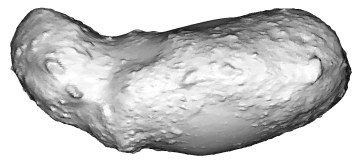

$\begin{aligned} m_{\mathbf{v}} & =39998 \\ \text { (e) } \quad m_{f} & =79992\end{aligned}$

$\bar{e}=1.3993$

Figure 4.8: (a)-(d) Models from the Standford scanning repository and (e) the asteroid Itokawa.

Figure 4.8 shows the models enlarged such that $\bar{e}=1$, then the armadillo, buddha, dragon, and Itokawa models are down-sampled to fewer faces and vertices using the MATLAB function reducepatch and MeshLab [12] to clean mesh discrepancies (e.g. non-manifoldness, repeated faces/vertices). Parameters common to the diffusion remain constant. For method DE: 15 runs per Monte Carlo simulation, $t_{0}=\bar{e} / 4, \alpha=1, k=1.2$, and $\eta=80$. For the mesh LBO, we selected $\rho=4$ and tested both geodesic and Euclidean distance metrics. For method GE: 5 runs per Monte Carlo, $\sigma=0.8 \bar{e}, \alpha=1 / 2, k=\sqrt{2}$, $\rho=4, N=10$, and tested both Euclidean and geodesic distance metrics. 


\subsubsection{Repeatability Metrics}

Our analysis uses three metrics of repeatability to determine the performance of each of the diffusion methods on explicit surfaces. The definitions are nearly identical to those presented in [132], but with a slight modification to the definition of repeatable keypoints. Begin with the set of all keypoints existing on the mesh that also exist in the scene that are not occluded as $\mathcal{K}=\left\{\mathbf{v}_{i}, \sigma_{i}\right\}_{i=1}^{m_{\mathcal{K}}}$ and the set of keypoints identified in the scene as $\mathcal{K}^{\prime}=\left\{\mathbf{v}_{i}^{\prime}, \sigma_{i}^{\prime}\right\}_{i=1}^{m_{\mathcal{K}}^{\prime}}$.

We define the set of repeatable keypoints as

$$
\begin{aligned}
\mathcal{K}_{R}=\left\{\mathbf{v}_{i} \in \mathcal{K}:\right. & \left(\left\|\mathbf{R}_{\mathcal{K}^{\prime}}^{\mathcal{K}} \mathbf{v}_{i}+\mathbf{t}_{\mathcal{K}^{\prime}}-\mathbf{v}_{i}^{\prime}\right\|<\ell_{d}\right) \\
& \left.\wedge\left(\sigma_{i / j}>\ell_{r}\right)\right\}
\end{aligned}
$$

where $\mathbf{R}, \mathbf{t}$ are the true rotation and translation and Eq. 4.24 defines $\sigma_{i / j}$. This is to say that the detected keypoint is repeatable if it lies within a distance threshold $\ell_{d}$ and a scale threshold $\ell_{r}$ of the currently visible model keypoints transformed into the measured frame. This definition differs slightly from that of [132] because it is possible to have multiple keypoints at the same physical location with sufficiently different scales to warrant distinct keypoint identification. We test two distance thresholds corresponding to those introduced in Section 4.2.2: $\ell_{d}=2 \bar{e}$ (similar to [132]) and $\ell_{d}=\sigma_{i} / 2$.

The remaining definitions follow the convention set in [132]. The first measure is that of absolute repeatability, which is the quantity of keypoints that exist in both the truth and noisy models

$$
r_{a b s}=\left|\mathcal{K}_{R}\right|
$$


where $|\cdot|$ is the returns the cardinality of the set. The quantity of repeatable keypoints should balance being sufficient large to facilitate a successful matching and pose estimation routine, but small enough to use computational resources efficiently.

The second measure is that of relative repeatability, which is the ratio between the quantity of repeatable keypoints and the quantity of keypoints in the truth model

$$
r_{\text {rel }}=\frac{\left|\mathcal{K}_{R}\right|}{|\mathcal{K}|} .
$$

The third measure is scale repeatability, which is the average percentage overlap of all pairwise matching keypoints. Given a repeatable keypoint $\left(\mathbf{v}_{i}, \sigma_{i}\right) \in \mathcal{K}_{R}$ and its closest match in $\left(\mathbf{v}_{j}, \sigma_{j}\right) \in \mathcal{K}^{\prime}$, the pairwise scale repeatability is

$$
r_{i j}=\frac{V\left(\operatorname{Sp}\left(\sigma_{i}\right)\right) \cap V\left(\operatorname{Sp}\left(\sigma_{j}\right)\right)}{V\left(\operatorname{Sp}\left(\sigma_{i}\right)\right) \cup V\left(\operatorname{Sp}\left(\sigma_{j}\right)\right)}
$$

where $\operatorname{Sp}(\sigma)$ is a sphere of radius $\sigma$ and $V(\mathrm{Sp})$ is the volume of the sphere. The overall scale repeatability for a single scene-to-model pair is

$$
r_{\sigma}=\left(\sum_{\substack{\mathbf{v}_{j} \in \mathcal{K}^{\prime} \\ \mathbf{v}_{i} \in \mathcal{K}_{R}}} r_{i j}\right) /\left|\mathcal{K}_{R}\right| .
$$

These three repeatability measures interact to determine the overall performance of the diffusion methods on explicit surfaces. Simply having a high value for a single repeatability measure does not indicate that the method is successful. For diffusion using discrete Laplacians, the objective is to find if 
any LBO performs comparably to or better than the other LBOs in all three repeatability measures. For diffusion using discrete Gaussians, the objective is to determine if the Euclidean distance metric is a sufficient approximation of the geodesic distance metric. In our experiments, we test two values for $\ell_{d}$ in Eq. 4.27. We noticed that with its variation, there is a noticeable and opposing trade-off between relative and scale repeatability.

\subsubsection{Keypoint Repeatability Results}

The first experiment determines repeatability when adding noise to the mean curvature signal while the vertex locations remain fixed. Various noise levels are considered as scalar multiples of the true ${ }^{7}$ mean curvature's standard

deviation. Gaussian white noise is added to the true mean curvature signal as

$$
\bar{\kappa} \leftarrow \bar{\kappa}+\nu \quad \nu \sim \mathcal{N}\left(0,\left(\eta \sigma_{\bar{\kappa}}\right)^{2}\right)
$$

where $\eta \in[0.1,0.2,0.3,0.4,0.5]$. Since the diffusion kernels (e.g. LBO or Gaussian) are calculated based on the underlying structure and are independent of the signal, they are only calculated once for this experiment and used for all the noise signal cases.

The second experiment determines repeatability when adding noise to the vertex locations and the mean curvature signal is reevaluated. Various noise levels are considered as scalar multiples of the average edge length, $\overline{\text { e. }}$ Gaussian white noise is added to the vertex locations along their normal vector

\footnotetext{
${ }^{7}$ Truth being the original mean curvature without added noise.
} 


$$
\mathbf{v} \leftarrow \mathbf{v}+\mathbf{n} \nu \quad \nu \sim \mathcal{N}\left(0,(\eta \bar{e})^{2}\right)
$$

where $\eta \in[0.1,0.2,0.3,0.4,0.5]$. The mesh triangulation remained constant and the mean curvature signal was estimated using MeshLab. Since the diffusion kernels depend on the mesh structure, they are recalculated for each run of the Monte Carlo experiments.

\subsubsection{Repeatability of DE adding Signal Noise}

The results shown in Fig. 4.9 are grouped by model. Each line shows the repeatability of that $\mathrm{LBO}$ as the noise level increases.

The umbrella LBO found the largest quantity of repeatable keypoints, mainly because it produces the most keypoints. The cotangent LBO produced the fewest quantity of repeatable keypoints. Despite having the largest absolute repeatability, the umbrella LBO does not perform as well as the mesh LBO in terms of relative and scale repeatability. The cotangent LBO performs the worst on all three metrics, made evident by the sharp decline in relative repeatability as signal noise increases. Relative and scale repeatability for all three LBOs exhibit the expected trend of degradation as the noise level increases. The mesh LBO has the most reliable performance for relative and scale repeatability. LBO convergence properties listed in Table 3.2 predicted the relative performance among the LBOs where stronger convergence correlates to better repeatability. Independent of the chosen LBO, repeatability metrics appear to also be affected by the chosen model. The quantity of 

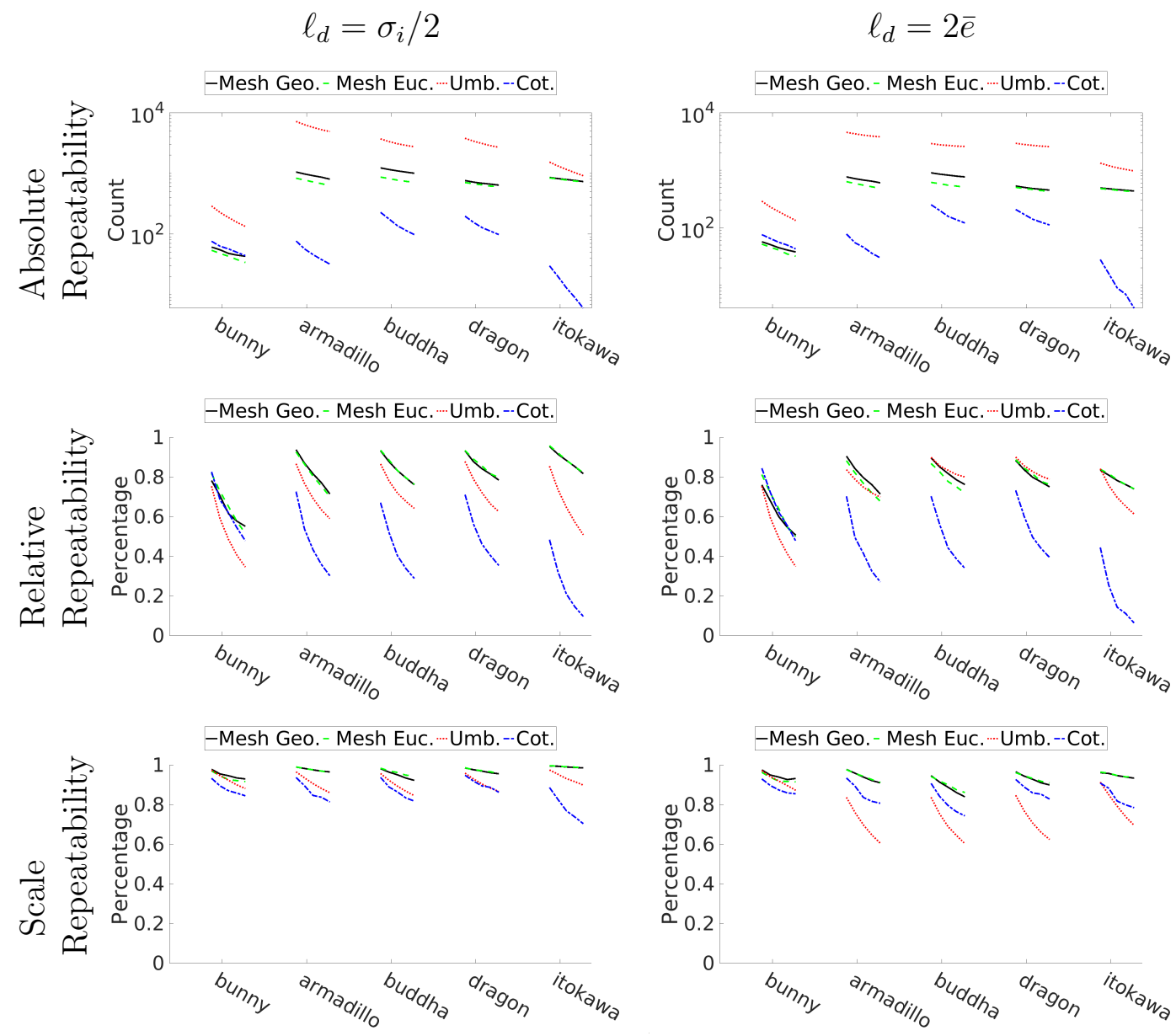

Figure 4.9: Keypoint repeatability results when adding Gaussian white noise to the signal. Median values for the Monte Carlo simulation are presented for the five levels of noise. Colors correspond to LBOs as the mesh LBO (geodesic metric) in solid black, mesh LBO (Euclidean metric) in dashed green, umbrella LBO in dotted red, cotangent LBO in dash dotted blue. For Eq. 4.27, the cases are $\ell_{d}=\sigma_{i} / 2$ (left column), or $\ell_{d}=2 \bar{e}$ (right column). 
repeatability keypoints are the lowest overall for the bunny model. Relative repeatability is also slightly lower overall for the bunny model, regardless of LBO.

Two cases for $\ell_{d}$ are shown in Fig. 4.9 to showcase the effects of different settings. In the top row, the umbrella operator performs better than the cotangent LBO, but worse than the mesh LBO, in terms of relative and scale repeatability. A different trend occurs in the bottom row where the umbrella LBO performs similarly to the mesh LBO in terms of relative repeatability, but then performs worse than the cotangent LBO on scale repeatability. Absolute repeatability remains highest for the umbrella LBO for both rows, and lowest for the cotangent LBO. While the mesh LBO finds an intermediate quantity of repeatable keypoints, its relative and scale repeatability are highest among all models and all LBOs.

The mesh LBO performs nearly identical for both the geodesic and Euclidean distance metrics across all three repeatability measures. The Euclidean mesh LBO performs only marginally lower than the geodesic mesh LBO in absolute repeatability for a few models. This shows that the Euclidean distance metric is sufficiently accurate to represent the mesh models in this work. The Euclidean mesh LBO is quicker to construct than the geodesic version, but is interchangeable with the geodesic mesh LBO without a loss in performance.

High rates of relative and scale repeatability occur as expected for each of the LBOs in the signal noise case since each exhibit some form of convergence $[115,116,119]$ and because the underlying mesh structure remains unaltered. 
Adding noise to the vertex locations will determine how the mesh LBOs react to a changing mesh structure.

\subsubsection{Repeatability of DE adding Vertex Noise}

Fig. 4.10 shows the results. For this experiment, the umbrella LBO was only calculated once because it only depends on connectivity of the mesh. The mesh and cotangent LBOs were recalculated for each run of the Monte Carlo simulation because they change depending on the mesh structure.

The values for the three repeatability measures decrease in this experiment as compared to the signal noise experiment. Similar to the first experiment, relative and scale repeatability for all three LBOs exhibit the expected trend of degradation as the amount of added noise increases. In the top row, the mesh LBO has the highest relative repeatability. The umbrella LBO performs at least as well as the cotangent LBO, but sometimes worse. All three LBOs have similar values for scale repeatability. Choice of model also affects repeatability performance, as the bunny model has the lowest values for absolute and relative repeatability regardless of LBO.

In contrast to the first experiment, the cotangent LBO performs at least as well as the umbrella LBO in all three repeatability measures. The difference in performance of the umbrella LBO may be attributed to its insensitivity to changing vertex locations since it remains constant based on connectivity. Conversely, the cotangent and mesh LBOs adjust to the changing mesh structure. 

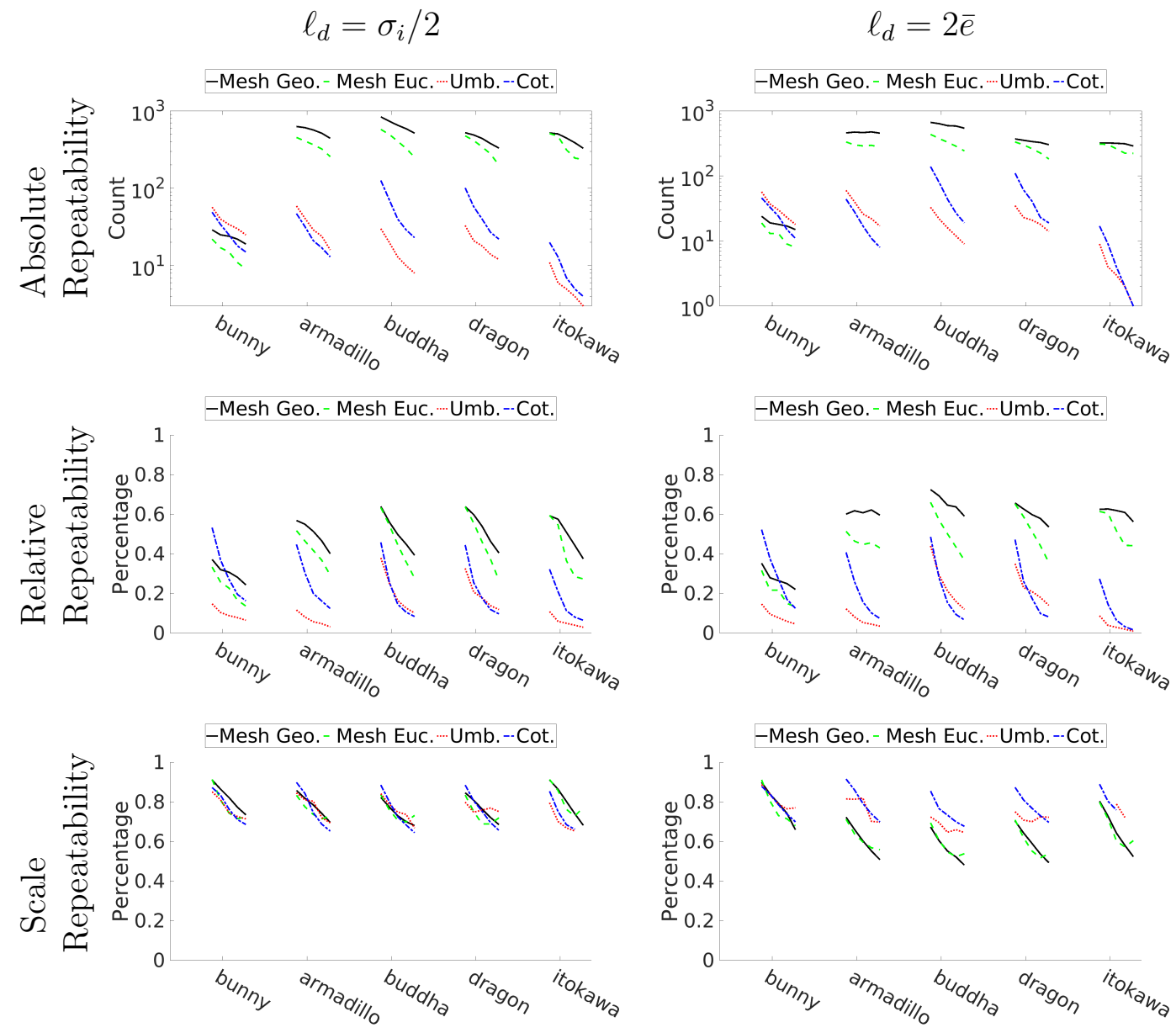

Figure 4.10: Keypoint repeatability results when adding Gaussian white noise to the vertex locations and recomputing the mean curvature signal. Median values for the Monte Carlo simulation are presented for the five levels of noise. Colors correspond to LBOs as the mesh LBO (geodesic metric) in solid black, mesh LBO (Euclidean metric) in dashed green, umbrella LBO in dotted red, cotangent LBO in dash dotted blue. For Eq. 4.27, the cases are $\ell_{d}=\sigma_{i} / 2$ (left column), or $\ell_{d}=2 \bar{e}$ (right column). 
In this experiment, the performance difference between the geodesic and Euclidean mesh LBO is noticeable. While the mesh LBO has higher absolute and relative repeatability than the other LBOs, the performance varies by evaluation metric. The geodesic mesh LBO performs slightly better than the Euclidean mesh LBO for absolute and relative repeatability. The mesh LBO performs nearly identical on scale repeatability for both geodesic and Euclidean. We consider the geodesic mesh LBO to be the most repeatable option for keypoint detection because it performs highest in absolute and relative repeatability, and similarly to the other LBOs on scale repeatability.

\subsubsection{Repeatability of GE adding Signal Noise}

The results shown in Fig. 4.11 are grouped by model. Each line shows the repeatability as the noise level increases for the two tested distance metrics. For this experiment, the Gaussian kernel is only calculated once per model and distance metric combination because the mesh structure remains constant while only the signal is changing.

Method GE performs equivalently for both distance metric selections on all three measures of repeatability. This shows that the additional resources required to compute the geodesic distance are unnecessary and may be well approximated by Euclidean distances. The overall quantity of keypoints is about an order of magnitude less than that of method DE. Repeatability is affected by (1) the level of noise, as shown by the negative sloping lines, and (2) the model. Unlike method DE, there is little affect seen by the two settings 

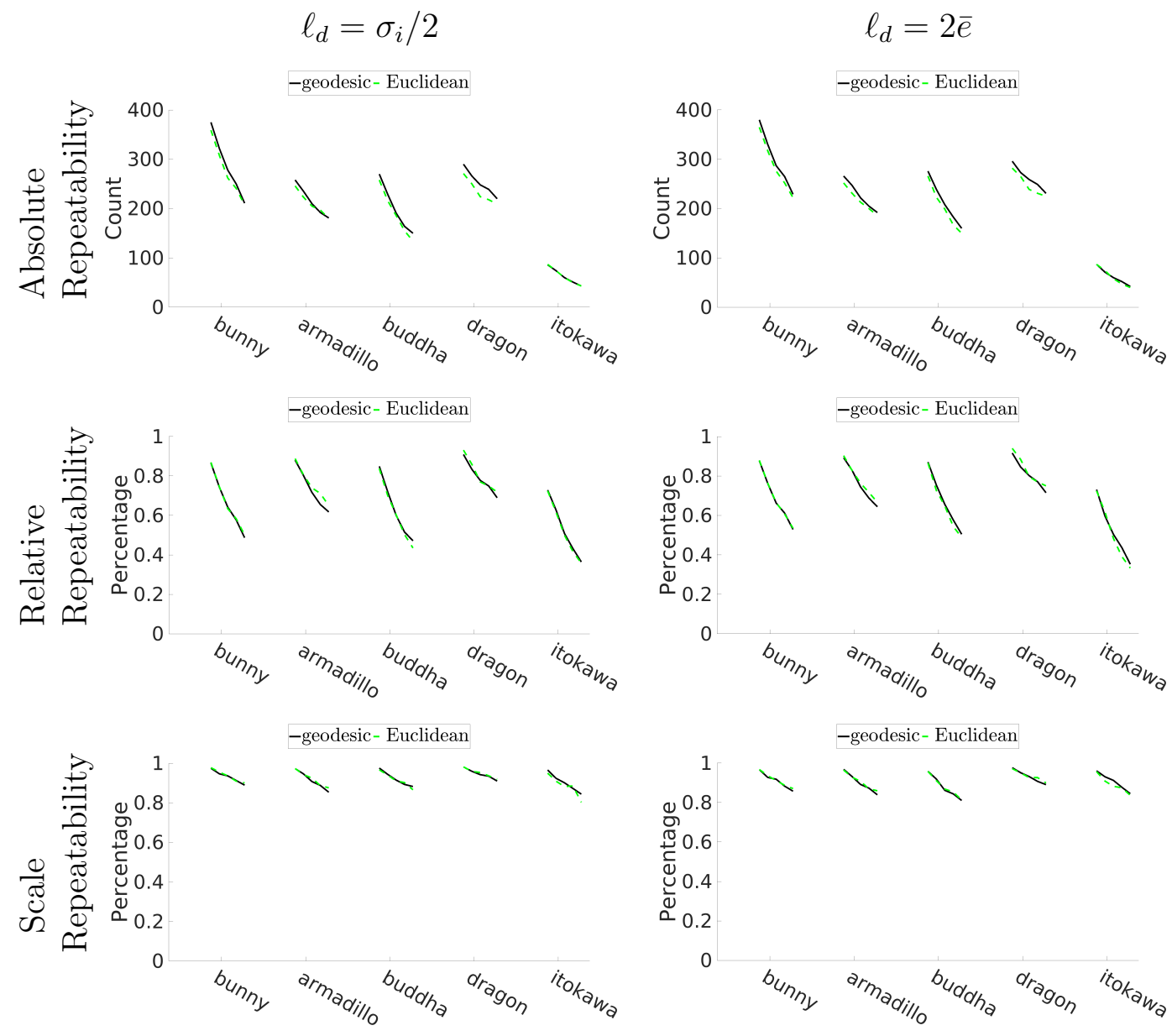

Figure 4.11: Keypoint repeatability results for Gaussian diffusion on meshes when adding Gaussian white noise to the signal. Median values for the Monte Carlo simulation are presented for the five levels of noise. Colors correspond to distance metric as geodesic in solid black and Euclidean in dashed green. For Eq. 4.27, the cases are $\ell_{d}=\sigma_{i} / 2$ (left column), or $\ell_{d}=2 \bar{e}$ (right column). 
of $l_{d}$.

Absolute repeatability of the bunny model degrades the quickest with increased noise, while remaining much more constant for the Itokawa model. Relative repeatability begins high for all models (except Itokawa), but degrades quickly with increased noise. Performance is in between that of method DE using the umbrella or cotangent LBO. Scale repeatability is high, nearly constant and identical for all models. Unfortunately, this is of only a marginal benefit given the low values of relative and absolute repeatability.

\subsubsection{Repeatability of GE adding Vertex Noise}

Results are shown in Fig. 4.12. For this experiment, the Gaussian kernel is recalculated for each Monte Carlo run since the underlying mesh structure is changing with the noise level.

All three measures of repeatability decreased relative to the previous signal noise experiment. Once again, performance is nearly equivalent for both the Euclidean and geodesic distance metrics. Absolute repeatability quickly

degrades with increased noise, with performance much worse than that of method DE in Fig. 4.10. With the lowest noise level, the quantity of keypoints is at least an order of magnitude less than method DE. With the highest level of noise, only a handful of keypoints are identified on each model.

Relative repeatability is low and close to that of method DE using either the umbrella or cotangent LBOs. Scale repeatability performs similarly for all models. Given the similarities between repeatability performance using either 

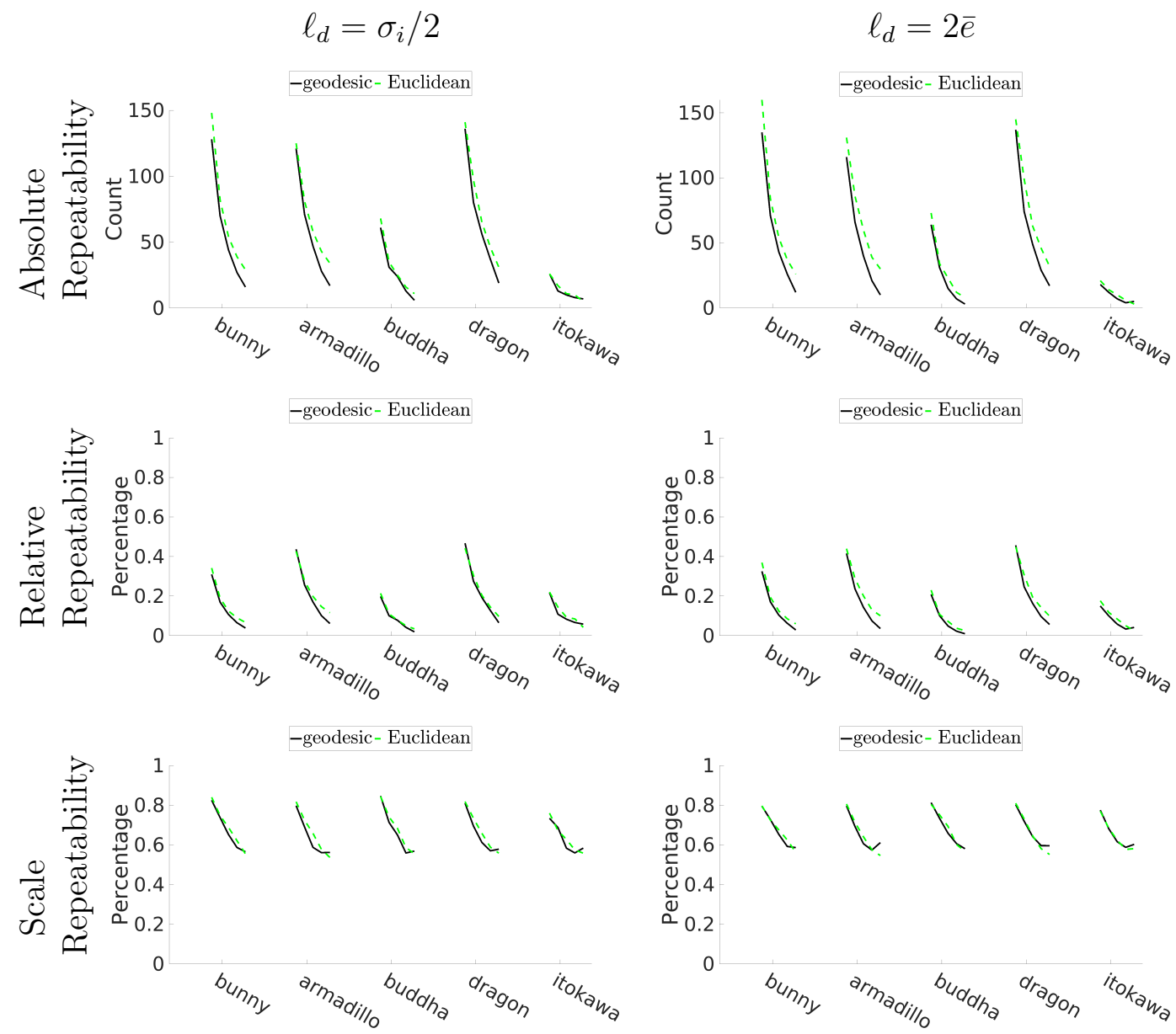

Figure 4.12: Keypoint repeatability results for Gaussian diffusion on meshes when adding Gaussian white noise to the vertex locations and recomputing the mean curvature signal. Median values for the Monte Carlo simulation are presented for the five levels of noise. Colors correspond to distance metric as geodesic in solid black and Euclidean in dashed green. For Eq. 4.27, the cases are $\ell_{d}=\sigma_{i} / 2$ (left column), or $\ell_{d}=2 \bar{e}$ (right column). 
the geodesic and Euclidean distance metrics, we suggest using the Euclidean distance because it is less computationally expensive to compute.

\subsection{Comments on Scale-Space Diffusion Methods}

Scale-space construction using two different diffusion methods were tested in this chapter. Method DE uses a discrete LBO while method GE uses a discrete Gaussian as the diffusion kernel. Theoretically, method DE is a first order approximation to method GE using backward Euler discretization in time; however, the three discrete LBOs discussed here have theoretical convergence to the true diffusion kernel $[116,119]$, while the discrete Gaussian does not. Some research has shown that discrete Gaussian construction on general explicit 3D surfaces may be futile [133]. The difference in theoretical support may contribute to the difference in repeatability performance. Another major difference between the methods is the separation between the scale-space levels. Method DE uses $k=1.2$ (but is user-defined) while method GE permanently selects $k=\sqrt{2}$ for convenience. While the affect of varying $k$ is not immediately evident, it may be of importance in scale-space construction.

Keypoint repeatability of our scale-space using both methods is tested using keypoint repeatability. The signal was arbitrarily chosen as mean curvature since it is independent of the selected sensor, but the scale-space is designed for any signal defined on the surface. Signals may be environment dependent (e.g. color or shading by lighting source) or artificially overlaid (e.g. image projected onto a surface). Given that these experiments used the 
mean curvature signal for diffusion, keypoint locations are understood to be extrema in mean curvature. Other signal types will result in keypoints at different locations and scales. 


\section{Chapter 5}

\section{Feature Histogram Descriptors}

The application of OUR-CVFH to the scenario of relative navigation (RelNav) to an asteroid is presented. We discuss the observability of the model Itokawa using OUR-CVFH which suggest certain areas of the model are unconducive to accurate pose estimation. An example rendezvous shows that OUR-CVFH offers pose estimates independent of the navigation filter. This successfully disconnects the external feedback loop from the output states to the pose estimation sensor as discussed in Sec. 1.1.

The use of scale is incorporated into the well-known feature histogram descriptor OUR-CVFH . Scale is used to quickly identify similar clusters during the matching procedure. This reduces run-time by only comparing probable clusters of similar size. Many other modifications are made to OUR-CVFH to improve pose estimation accuracy and robustness to noise, measurement error, and sensor resolution variation with feature interpolation, normalization, and introducing an new cost metric. This modifications take place in Sec. 5.2.

Portions of this chapter were previously published in [16]. Notation and terminology has been updated to maintain consistency and clarity of this document. 


\subsection{Application of OUR-CVFH to LIDAR-Based Rela- tive Navigation}

\subsubsection{Training}

Training is accomplished using a virtual 3D model and a LIDAR simulator. This option is prefferable to using a physical objects and a LIDAR

- especially for space applications where it is difficult to gather adequate training data on the actual object. A particularly good open source LIDAR simulator called GLIDAR [13] was used by Woods et al. [30] for demonstrations of RelNav with respect to non-cooperative objects in space. We utilize an in-house built Matlab LIDAR simulator.

We propose a different training strategy that OUR-CVFH discussed in Section 2.2.5.4. To create a database of OUR-CVFH histograms, we place a 3D model at the origin of a polyhedron with equally spaced vertices. The vertices must be evenly spaced so that all orientations of the model are equally seen without favoring any particular set of orientations. The number of vertices of the polyhedron is application dependent, though a polyhedron with 20 vertices is usually sufficient. Training images must be taken at a variety of ranges, which are selected based on the intended operating range. The simulated 3D sensor is then placed at each of the vertices of the polyhedron at each of the training ranges. This method of training ensures that the object has been seen at a variety of ranges and attitudes. Selecting training ranges is application dependent and should be selected based on the intended operating ranges. Additionally, the training range intervals are incremented by steps equal to 
one-fourth the size of the model.

To complete the training stage, an additional pose must be stored for future use in pose estimation. To enable the estimation of pose from the training sensor to the active sensor, the camera pose $\mathbf{T}_{S}^{B}$.

\subsubsection{Recognition Framework}

The pose estimation framework of OUR-CVFH returns the relative $6 \mathrm{DOF}$ pose from the object body in the model frame $B_{\mathcal{M}}$ to the object body in the scene frame $B_{\mathcal{S}}$. This type of pose estimate would be good in a dual inertial state Kalman Filter [30]. While it is only a matter of preference, some may desire the $6 \mathrm{DOF}$ pose from the training camera instead of the model body. In this case the ICP output of Sec. 2.2.5 Step 2 is multiplied by the true training pose of the sensor $\mathbf{T}_{S}^{B}$ to yield

$$
\widetilde{\mathbf{T}}_{S}^{B}=\delta \widetilde{\mathbf{T}}_{S}^{+} \mathbf{T}_{S}^{B}
$$

which is the relative pose estimate from the training camera pose the active sensor pose.

\subsubsection{Uniqueness of Feature Histograms and Pose Observability}

Recall that one of the requirements for using OUR-CVFH is that the object has sufficient uniqueness in shape and/or texture. Let us consider the observability of an object as viewed from different directions by assessing the accuracy of the pose measurement returned by OUR-CVFH . The pose estimate could be faulty for two reasons: (1) the OUR-CVFH descriptor is not 


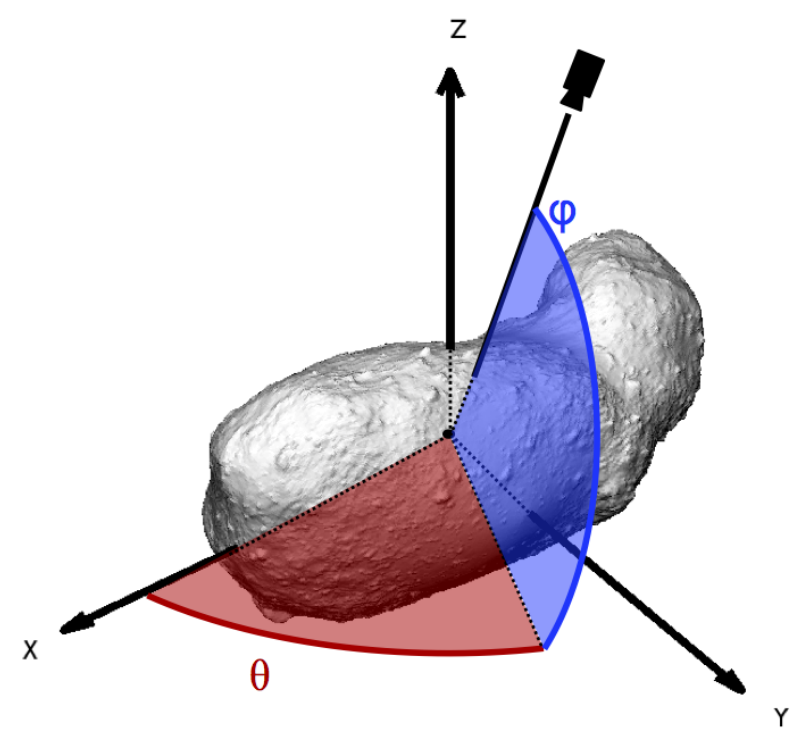

Figure 5.1: Reference frame for Itokawa. Azimuth and elevation angles are defined from the $x$-axis. Use this image to aid in reading Fig. 5.2.

sufficiently unique to distinguish clusters or objects or (2) the object exhibits poor shape and/or texture uniqueness at specific ranges or orientations.

As an illustrative example, consider the pose observability of the asteroid Itokawa. Here, we will assess the observability at a range of 300 meters by finding the pose performance for a sensor placed at various combinations of azimuth, $\theta$, and elevation, $\phi$, angles. These angles are defined in Fig. 5.1 and were sampled at intervals of $4^{\circ}$ each. The colored scatter plots of attitude (top) and position (bottom) errors produced from the OUR-CVFH pose estimate are shown in Fig. 5.2. It is immediately evident that the majority of viewpoints of the asteroid provide good pose estimates (there is much more blue than red in these plots) and that some viewpoints have better observability 
than others. Poorer viewpoints are those that generate larger pose estimation errors.

There are viewing regions of Itokawa where OUR-CVFH produces accurate pose estimates. For example, the regions surrounding $(\theta, \phi)=(90,0)$ and $(270,0)$ in Fig. 5.2 provide especially good viewing geometry for two reasons. First, the surface area of Itokawa visible to the LIDAR in these regions is abundant, which permits numerous clusters - more clusters in a particular view yield more opportunities for correct histogram matching. Second, the surface of Itokawa visible at these attitudes has sufficiently unique shape and texture to create uniquely descriptive histograms. Approaching Itokawa from these orientations would provide the most accurate pose estimates.

On the contrary, OUR-CVFH produces a poor pose estimate in the regions surrounding $(\theta, \phi)=(180,-45)$ and $(360,0)$. The surface area of Itokawa visible to the LIDAR in these attitude regions is minimal and rough, leading to a few small clusters that are not sufficiently unique to correctly identify. When designing an approach trajectory towards Itokawa, it would be advisable to avoid these relative attitudes which are known to produce poor pose estimates.

Thus, for this example, the OUR-CVFH histogram descriptor is sufficiently unique to identify clusters from neighboring viewpoints as shown in the regions of Fig. 5.2 having accurate pose estimates (the blue regions). Generalizing this result, we observe that the surface of the object visible to the LIDAR must be sizable and distinctive to form unique histograms. If the 

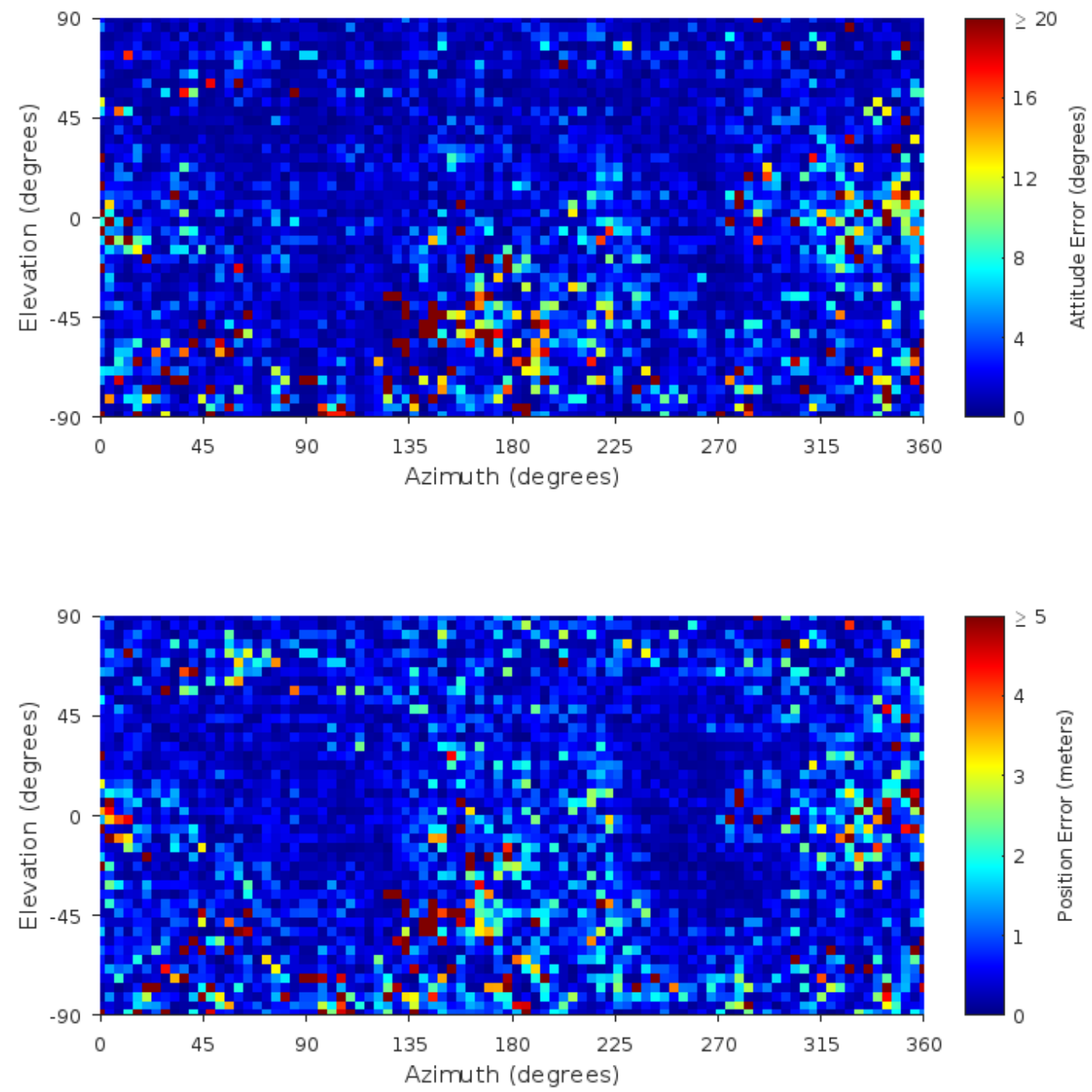

Figure 5.2: Attitude (top) and position (bottom) errors produced from OURCVFH pose estimation. Blue is an accurate estimate with nearly no error. Position errors of 5 meters or more, and attitude errors of $20^{\circ}$ or more are shown as dark red. Azimuth and elevation angles are defined from the $x$-axis as shown in Fig. 5.1. (This figure is best viewed in color.) 
viewing geometry limits the visible surface area, and this area is excessively rough, then OUR-CVFH is more likely to generate an inaccurate pose estimate. Improving the accuracy of pose estimation from OUR-CVFH could be accomplished by (1) making a more discriminating descriptor possibly using scale and/or (2) modifying the matching routine to include multiple clusters in a particular view that are geometrically consistent.

\subsubsection{Numerical Example: Rendezvous with an Asteroid 5.1.4.1 Problem Scenario and Monte Carlo Set-up}

As an illustrative example, consider a simple rendezvous with an asteroid. The specific scenario explored here is an approach to the asteroid Itokawa, beginning at a range of $350 \mathrm{~m}$ and ending at a range of $250 \mathrm{~m}$. A simulated Flash LIDAR of size $128 \times 128$ pixels and a $10^{\circ}$ FOV generates point clouds where each point is corrupted by Gaussian noise with a standard deviation of $\sigma=0.05$ meters. Point clouds are produced once every second $(1 \mathrm{~Hz})$ and are

processed by a Multiplicative Extended Kalman Filter (MEKF). The initial state uncertainty in the MEKF is $25 \mathrm{~m}$ in position, $5 \mathrm{~cm} / \mathrm{s}$ in velocity, 10 deg in attitude, and $0.06 \mathrm{deg} / \mathrm{sec}$ in body rate. A small Monte Carlo analysis is performed to demonstrate the performance of both OUR-CVFH and the MEKF. 


\subsubsection{Unfiltered OUR-CVFH Pose Performance}

The OUR-CVFH method computes the relative position and relative attitude between the sensor and the object directly from the 3D point cloud without the need of an a priori pose estimate. Thus, the use of OUR-CVFH to generate LIDAR-based pose estimates completely severs feedback of the navigation state into the measurement generation process. This is one of the primary benefits of OUR-CVFH .

The OUR-CVFH procedure outlined in Sec. 2.2.5 with the training from Sec. 5.1.1 was implemented by the authors in MATLAB and applied to the Itokawa rendezvous example described above. Examples of the automatically generated OUR-CVFH clusters along this approach trajectory are shown in Fig. 5.3. Additionally, the pose measurement residuals across all Monte Carlo runs are shown in Fig. 5.4.

\subsubsection{Processing with a Simple Multiplicative Extended Kalman Filter (MEKF)}

The raw OUR-CVFH pose measurements (whose errors are presented in Fig. 5.4) may be smoothed considerably using a simple MEKF. Classical Kalman filters are statistically optimal sequential estimators for systems with linear dynamics and linear measurement models [134, 135]. The extended Kalman filter (EKF) modifies the linear Kalman filter to handle both non-

linear dynamics and nonlinear measurement models [136]. The multiplicative EKF (MEKF) provides further modifications for intelligently handling attitude 

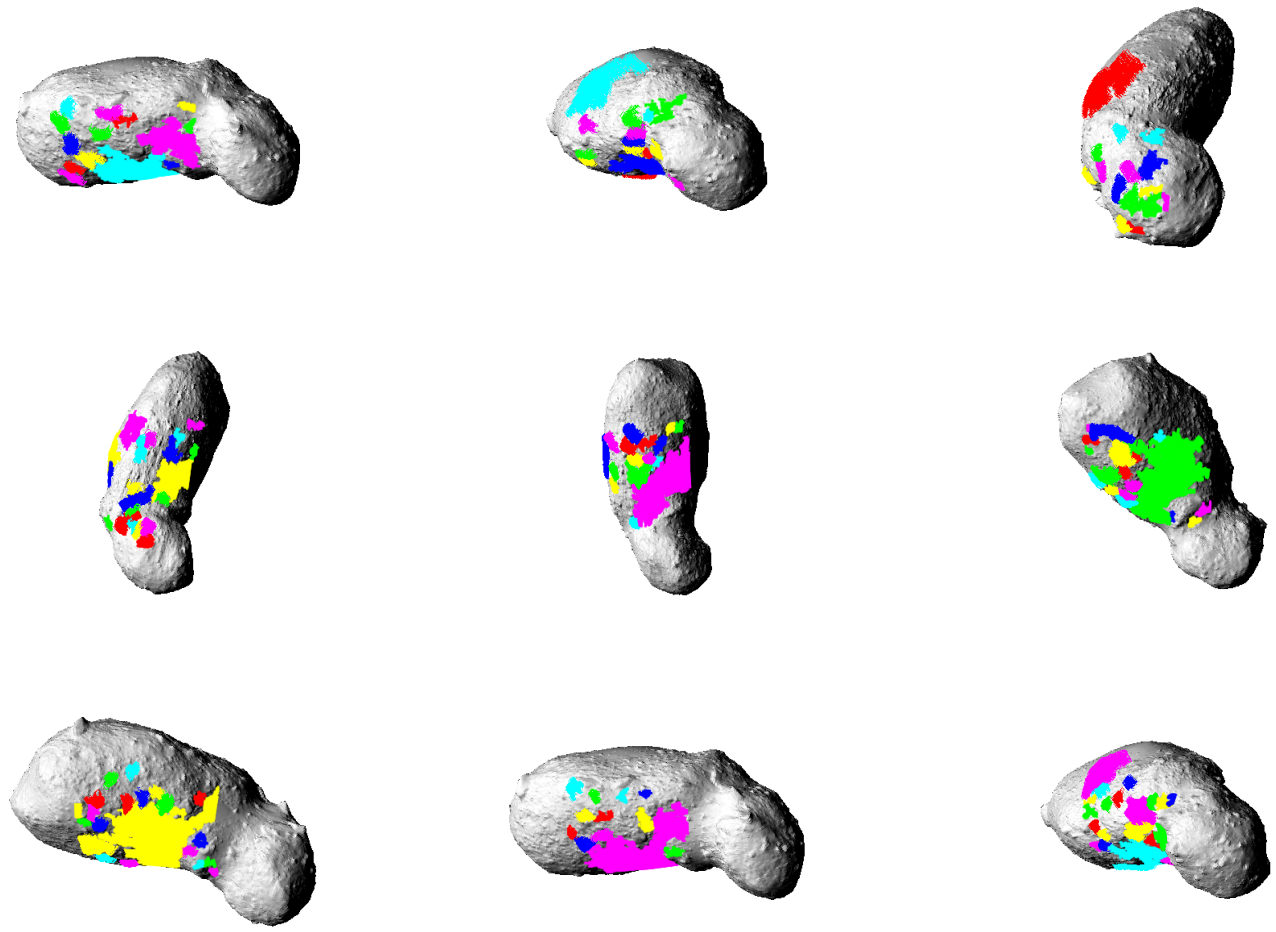

Figure 5.3: Example OUR-CVFH clusters along simulated rendezvous trajectory with Itokawa. Each cluster is represented by a different color. If two clusters have the same color but are clearly separated, then they indicate different clusters. 

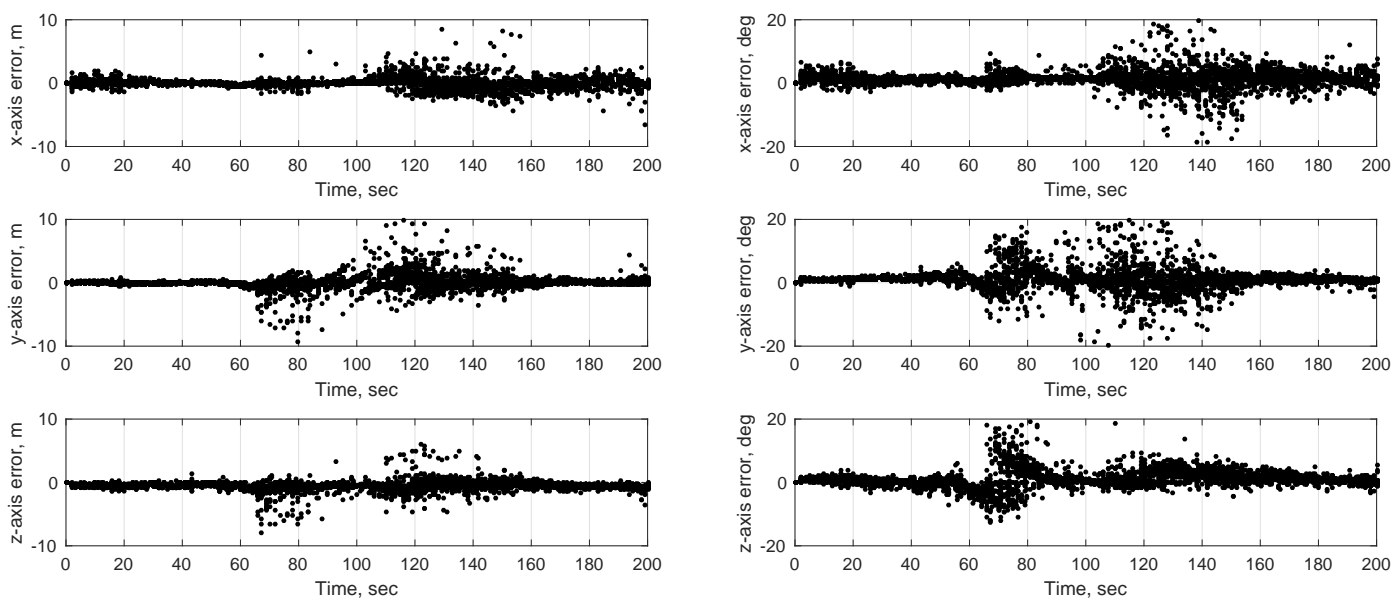

Figure 5.4: Relative position error (left) and relative attitude error (right) for raw OUR-CVFH pose results during simulated rendezvous with Itokawa for all Monte Carlo runs. The varying observability as the viewpoint changes is evident in the growing and shrinking of the residuals.

states $[137,138]$. Since the current problem considers both relative position and relative attitude, an MEKF was selected to filter the LIDAR-based pose measurements generated by OUR-CVFH .

\subsubsection{Brief Review of MEKF Implementation}

The MEKF state vector was chosen to be the $12 \times 1$ vector

$$
\mathbf{X}^{\top}=\left[\begin{array}{llll}
\mathbf{r}^{\top} & \dot{\mathbf{r}}^{\top} & \mathbf{a}^{\top} & \boldsymbol{\omega}_{r}^{\top}
\end{array}\right]
$$

where $\mathbf{r}$ is the relative position, $\dot{\mathbf{r}}$ is the relative velocity, $\mathbf{a}$ is the $3 \times 1 \mathrm{MEKF}$ attitude error vector, and $\boldsymbol{\omega}_{r}$ is the relative angular velocity. We find it necessary to estimate $\boldsymbol{\omega}_{r}$ since the asteroid is tumbling at an unknown rate (the inertial body rate of the spacecraft is assumed to be known from IMUs). 
Both the state estimate, $\hat{\mathbf{x}}$, and state covariance, $\mathbf{P}$, are propagated from the time of one measurement to another using the typical EKF approach

$$
\begin{gathered}
\dot{\hat{\mathbf{x}}}=f(\mathbf{x}, t) \\
\dot{\mathbf{P}}=\mathbf{F} \mathbf{P}+\mathbf{P F}^{\boldsymbol{\top}}+\mathbf{Q}
\end{gathered}
$$

where $\mathbf{F}=\partial f(\mathbf{x}, t) / \partial \mathbf{x}$ and $\mathbf{Q}$ is the process noise. In parallel, the estimated attitude quaternion, $\hat{\mathbf{q}}$, is also propagated using standard quaternion kinematics

$$
\dot{\hat{\mathbf{q}}}=\frac{1}{2}\left[\begin{array}{c}
\boldsymbol{\omega} \\
0
\end{array}\right] \otimes \hat{\mathbf{q}}
$$

where $\otimes$ is the quaternion multiplication operator.

Proceed by defining the OUR-CVFH based pose measurement available at time $t_{k}$ as

$$
\mathbf{y}_{k}=h\left(\mathbf{x}_{k}\right)=\left[\begin{array}{c}
\mathbf{r}_{k} \\
\mathbf{a}_{k}
\end{array}\right]
$$

This may be used to update the covariance at time $t_{k}$ using the Joseph form

$$
\mathbf{P}_{k}^{+}=\left(\mathbf{I}-\mathbf{K}_{k} \mathbf{H}_{k}\right) \mathbf{P}_{k}^{-}\left(\mathbf{I}-\mathbf{K}_{k} \mathbf{H}_{k}\right)^{\top}+\mathbf{K}_{k} \mathbf{R}_{k} \mathbf{K}_{k}^{\top}
$$

where $\mathbf{H}=\partial h(\mathbf{x}) / \partial \mathbf{x}$ is the measurement sensitivity matrix and $\mathbf{K}_{k}$ is the Kalman gain,

$$
\mathbf{K}_{k}=\mathbf{P}_{k}^{-} \mathbf{H}^{\top}\left(\mathbf{H P}_{k}^{-} \mathbf{H}^{\top}+\mathbf{R}_{k}\right)^{-1}
$$

The '-' superscript indicates the a priori estimate and the ' + ' superscript indicates the a posteriori estimate. 
The state update at time $t_{k}$ is performed in two steps. First, the nonattitude states are updated as in a typical EKF,

$$
\hat{\mathbf{x}}_{k}^{+}=\hat{\mathbf{x}}_{k}^{-}+\mathbf{K}_{k}\left(\tilde{\mathbf{y}}_{k}-h\left(\hat{\mathbf{x}}_{k}^{-}\right)\right)
$$

where $\tilde{\mathbf{y}}$ is the noisy OUR-CVFH measurement. Then, the attitude is updated multiplicatively using the attitude error state,

$$
\hat{\mathbf{q}}_{k}^{+}=\mathbf{q}\left(\hat{\mathbf{a}}_{k}^{+}\right) \otimes \hat{\mathbf{q}}_{k}^{-}
$$

The occasional OUR-CVFH pose outlier (especially in regions of poor observability) requires that a residual edit check be implemented to prevent the filter from processing erroneous measurements. This was done through a simple $\chi^{2}$ test using the measurement residual and the filter covariance. This check is straightforward to implement as

$$
\left(\tilde{\mathbf{y}}_{k}-h\left(\mathbf{x}_{k}^{-}\right)\right)^{\top}\left(\mathbf{H P}_{k}^{-} \mathbf{H}+\mathbf{R}_{k}\right)^{-1}\left(\tilde{\mathbf{y}}_{k}-h\left(\mathbf{x}_{k}^{-}\right)\right) \leq t o l
$$

While the appropriate value of tol varies from one scenario to another, the results presented in this paper assume a $4 \sigma$ threshold: tol $=4^{2}=16$.

\subsubsection{Filtered OUR-CVFH Pose Performance}

The MEKF described in the preceding section was implemented using the raw OUR-CVFH data presented in Sec. 5.1.4.2. The specific implementation used here assumed a constant covariance for the LIDAR measurement, with a standard deviation of 5 deg in relative attitude and a standard deviation of $5 \mathrm{~m}$ in relative position. The results for relative position error and 

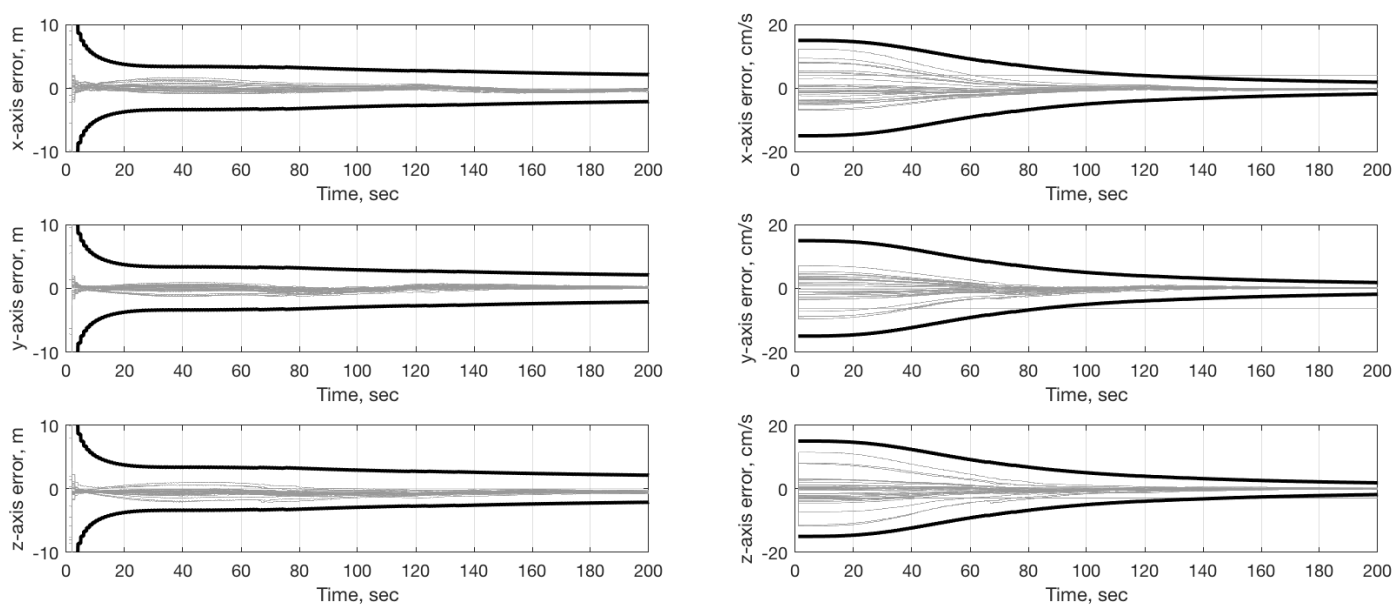

Figure 5.5: Relative position error (left) and relative velocity error (right) from the MEKF. The thin gray lines show results from individual Monte Carlo cases. The thick black lines show the $3 \sigma$ covariance bounds from the filter.

relative velocity error are shown in Fig. 5.5. Likewise, the results for the relative attitude error and relative body rate error are shown in Fig. 5.6.

The filtered results confirm that OUR-CVFH may be used to perform 6DOF RelNav with respect to a tumbling asteroid. Of particular note is that each and every OUR-CVFH pose estimate was generated without using any information from the filter, thus removing the filter feedback loop used in many LIDAR-based RelNav schemes.

\subsubsection{Comments on the use of OUR-CVFH in Space Applications}

The OUR-CVFH descriptor is well suited for spacecraft RelNav with respect to both natural and artificial objects. The primary requirements for using OUR-CVFH are as follows. First, the object must have a sufficiently 

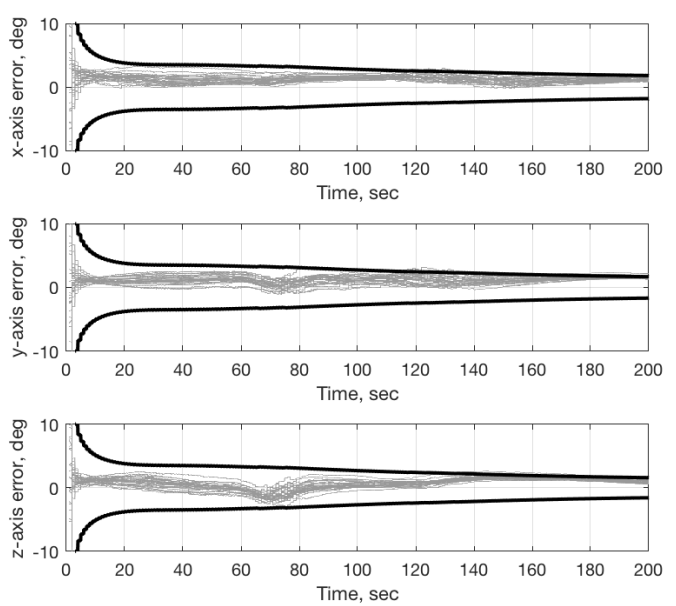
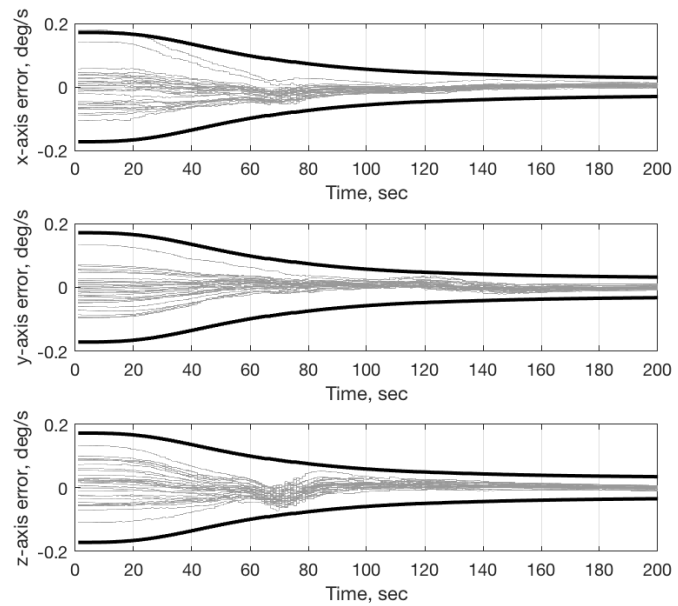

Figure 5.6: Relative attitude error (left) and relative body rate error (right) from the MEKF. The thin gray lines show results from individual Monte Carlo cases. The thick black lines show the $3 \sigma$ covariance bounds from the filter.

unique shape and/or texture and 3D point clouds must be collected from vantage points where this uniqueness is evident. Range images at very close range, for example, may not contain enough unique content to match the point cloud to the database. Second, an a priori $3 \mathrm{D}$ computer model of the object must exist for training. Such models typically exist for man-made satellites, and the generation of 3D models is a standard science data product for missions to asteroids, comets, and other natural bodies. Third, sufficient onboard computing resources must be available to run OUR-CVFH in real time. Fortunately, OUR-CVFH is highly parallelizable and may be accelerated in hardware through a filed programmable gate array (FPGA). Preliminary results [19] indicate that real-time implementation of OUR-CVFH is possible.

Recall that VFH, CVFH, and OUR-CVFH all assume a segmented 
scene. While complicated for cluttered office scenes and other personal robotics applications, scene segmentation is trivial for most space applications. Segmentation of objects in space is straightforward since the object of interest is typically surrounded by space (which appears to be at an infinite range and from which no signal is returned). Furthermore, the object of interest is typically the only object that is both within a detectable range and in the sensor's field of view.

The textbook implementation of OUR-CVFH assumes that the operational range between the 3D sensor and the observed objects does not greatly vary. Consequently, the spatial resolution of the measured scene remains reasonably constant. Since we intend to use OUR-CVFH for spacecraft RelNav which has varying operational ranges, the assumption of a constant resolution of the measured point cloud is not valid. Our measured point cloud will have widely different spatial resolutions depending on the actual range to the object. Therefore, when creating a training database according to the procedure in Sec. 2.2.5.4, we have to consider the many viewing orientations as well as multiple ranges. This updated training method is detailed in Sec. 5.1.1. In this way, our training procedure is different from $[79,54]$ as they consider training from multiple viewpoints but only at a single constant range.

Aldoma et al. [54] claim that since the height of the OUR-CVFH histogram is directly related to the number of points in the measured scene, the descriptor implicitly relays information about the object's scale. However, their conclusion is dependent on the assumption of a common spatial 
resolution between the model and measured point clouds. Since there is no explicit consideration of scale — and the upcoming thought experiment shows OUR-CVFH' 's failure in determining scale — the cost metric in Sec. 2.2.5.5 attempts to predominately match clusters of similar size. Though the overall OUR-CVFH generation and matching procedure does not directly address scale, satisfactory pose estimation is achieved while leaving capacity for future improvement.

A simple thought experiment shows the limitation in using histogram size as an implicit measure of feature scale. Consider a clustered point cloud of specific shape that has $n$ points with a physical size of $d$. The size may be different for the $x, y, z$ directions. Create an OUR-CVFH histogram from this cluster using features from Sec. 2.2.5. Now consider the same clustered point cloud of specific shape that still has $n$ points but now has size $2 d$. Once again, create its OUR-CVFH histogram. Since both point clouds have the same number of points $n$, they will have the same height. Also, by realizing that the OUR-CVFH features are all generated with unit vectors, the dimension of the object (either $d$ or $2 d$ ) will not affect the results of those sections of the histogram. Also notice that since the $I S O C$ histogram is normalized by the distance between the cluster centroid and farthest point in the cluster, this component will also remain the same for both point clouds. Therefore, there is a notable difficulty when considering the scale of an object from the histogram descriptor, and in fact, all of the descriptors presented here are scale invariant. This deficiency does not prevent OUR-CVFH from being used 
for spacecraft RelNav, and in fact, still shows accurate results in Sec. 5.1.4. However, this issue should be addressed in future innovations of the feature histogram descriptor. A feature histogram descriptor that explicitly characterizes the scale of a cluster would be beneficial to the aerospace community for spacecraft RelNav.

An additional benefit of OUR-CVFH is that of object recognition. Aldoma et al. [54] originally intended OUR-CVFH to be utilized by robots in identifying different objects that lie in a relatively cluttered environment (e.g. in an office, kitchen, or industrial workbench). For spacecraft in the RelNav environment, the on-board training database could contain model feature histograms for multiple objects. Then when encountering any one of those objects, the OUR-CVFH recognition process will automatically determine which object from the database is being observed as well as the 6DOF relative pose of that object.

\subsection{Development of Scaled OUR-CVFH (SOUR-CVFH)}

This section details our modifications to OUR-CVFH in development of SOUR-CVFH . We present the modifications individually in order to show the effects of each step. The final SOUR-CVFH descriptor is described concisely in Section 5.3. In this section, we specifically address the possible improvements discussed in Section 5.1.5 of (1) directly addressing a cluster's physical scale which is directly related to (2) reducing the required computational resources. Additional modifications to increase the performance are made and 
separated by section. Section 5.2.1 discusses histogram component interpolation, Section 5.2.2 evaluates the description space of specific components, Section 5.2.3 investigates a new cost metric function, Section 5.2.4 normalizes the histogram, Section 5.2.5 compares multiple distance functions for matching histograms, Section 5.2.6 develops of method of estimating and comparing cluster scale, Section 5.2.7 compares weighting techniques for the spatial feature description, Section 5.2.8 compares the performance of different feature combinations, and Section 5.2.9 modifies the manner in which the cluster reference frame is selected. Some of these contributions are shown graphically in Fig. 5.7. Each section is followed by an example on how our adjustment modifies the OUR-CVFH descriptor and its effect on pose estimation.

The experiments in the following section are performed using the same set of training data, unless otherwise noted. Training is performed following the procedure in Section 2.2.5.4 with the following specific characteristics. The LIDAR FOV is $20^{\circ}$ with a resolution of $128 \times 128$. There are 642 unique viewpoints evenly space around the Itokawa model shown in Fig. 4.8, at ranges between 200 meters and 400 meters at 25 meter intervals. The same set of Monte Carlo experimentation data is also used for each experiment, unless otherwise noted. A 500 run Monte Carlo is performed with attitude quaternions and ranges individually sampled from uniform distributions. The sampled range are within the 200-400 meter training range. 

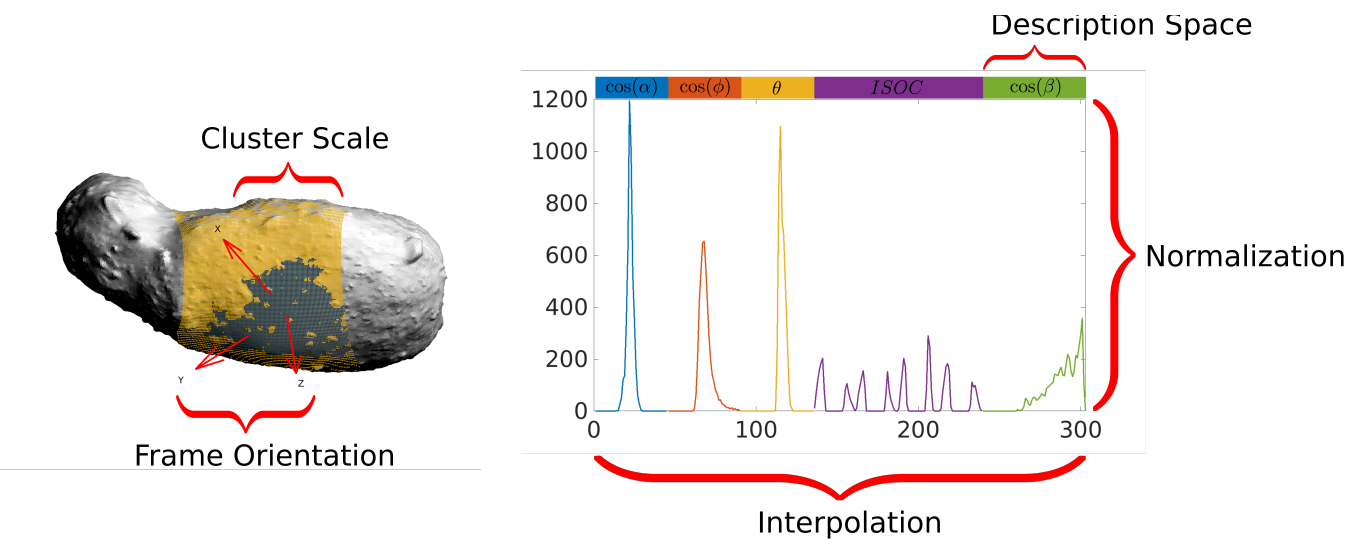

Figure 5.7: Visual indications of modifications made to OUR-CVFH to develop SOUR-CVFH and SOL-CVFH . Section 5.2.1 discusses interpolation, Section 5.2.2 discusses description space, Section 5.2.4 discusses histogram normalization, Section 5.2.6 discusses cluster scale, and Section 5.2.9 discusses reference frame orientation.

Table 5.1: Statistics for the 500 run Monte Carlo experiment comparing linear interpolation of angular distributions to OUR-CVFH . Method A uses LERP for $\cos \alpha_{i}, \cos \phi_{i}, \theta_{i}$, and $\cos \beta_{i}$. Method A performs better on every statistic except for Num. Pose Estimates and median angle error.

\begin{tabular}{|c|c|c|}
\hline & OUR-CVFH & A \\
\hline \multicolumn{3}{|c|}{ Num. Pose Estimates } \\
\hline & 470 & 469 \\
\hline \multicolumn{3}{|l|}{ Angle Error } \\
\hline Mean (deg.) & $9.78^{\circ}$ & $8.40^{\circ}$ \\
\hline Median (deg.) & $2.44^{\circ}$ & $2.49^{\circ}$ \\
\hline Std. Dev. (deg.) & $29.12^{\circ}$ & $27.08^{\circ}$ \\
\hline \multicolumn{3}{|l|}{ Range Error } \\
\hline Mean (m.) & $3.59 \mathrm{~m}$ & $3.18 \mathrm{~m}$ \\
\hline Median (m.) & $1.43 \mathrm{~m}$ & $1.38 \mathrm{~m}$ \\
\hline Std. Dev. (m.) & $8.52 \mathrm{~m}$ & $8.21 \mathrm{~m}$ \\
\hline
\end{tabular}




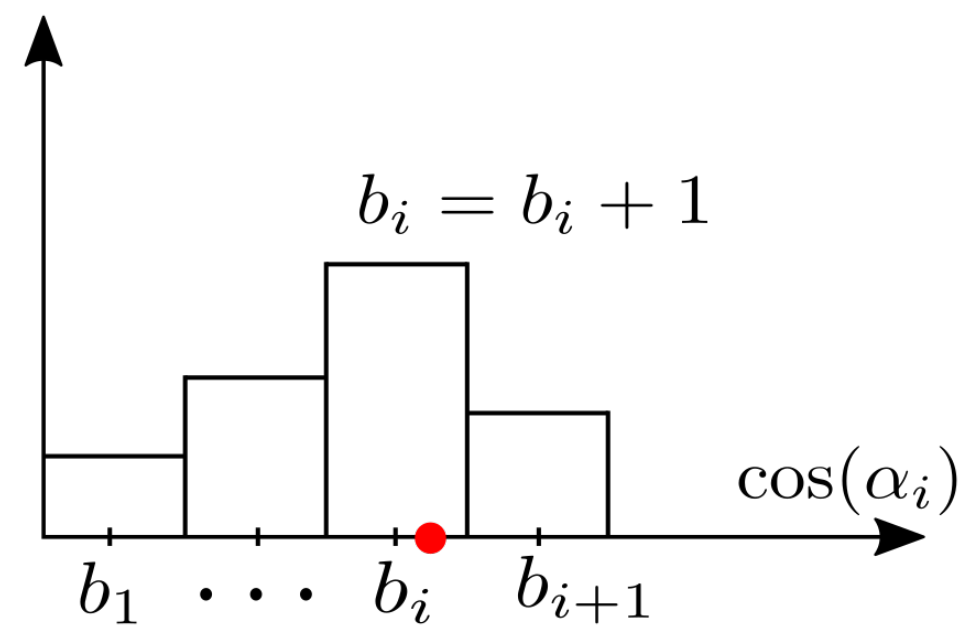

(a) OUR-CVFH bin accumulation

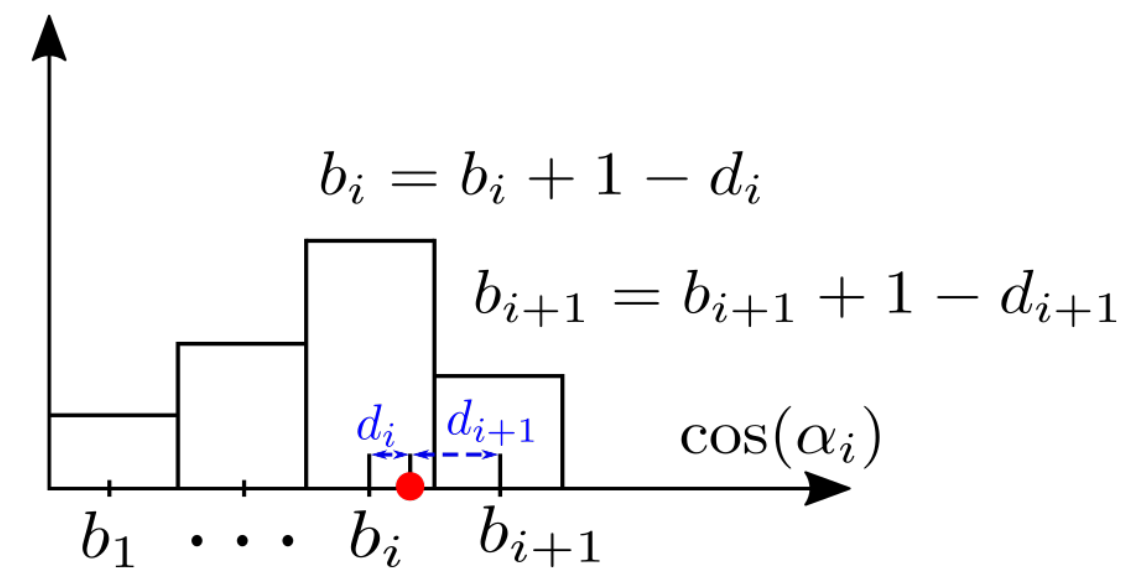

(b) LERP bin accumulation

Figure 5.8: Bin accumulation comparison for OUR-CVFH and the proposed LERP. The red dot is a value to be binned. In OUR-CVFH, the value completely lies within bin $b_{i}$. Using LERP, the value is distributed across bins $b_{i}$ and $b_{i+1}$ depending on the distance to the bin centers. 


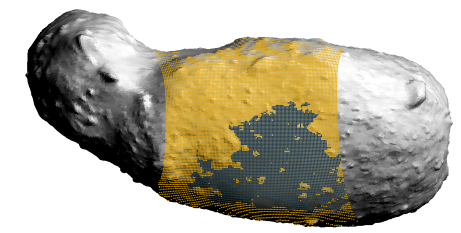

(a) Itokawa with scene and cluster

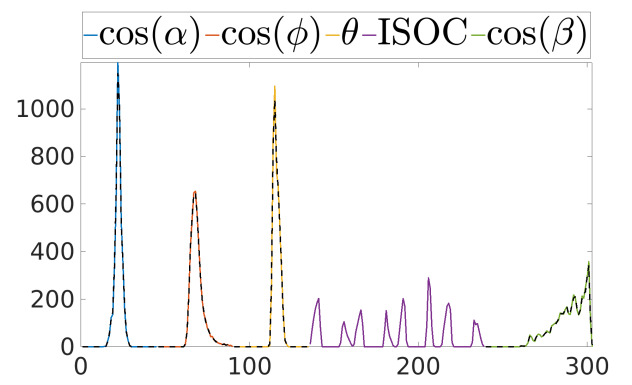

(b) Histogram with and without LERP

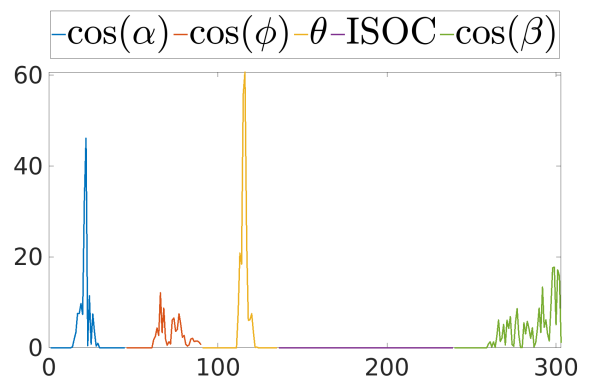

(c) Difference between histograms

Figure 5.9: Visual affects of LERP on histogram construction. (a) Itokawa with the measured scene shown in yellow and cluster of interest shown in blue. (b) OUR-CVFH histogram shown in colors corresponding to histogram components. LERP of components overlaid in black dashed line. (c) Absolute difference between histograms with and without LERP color coded by component. Notice that only $\cos \left(\alpha_{i}\right), \cos (\phi), \theta$, and $\cos \left(\beta_{i}\right)$ differ between histograms. 
Angle Error
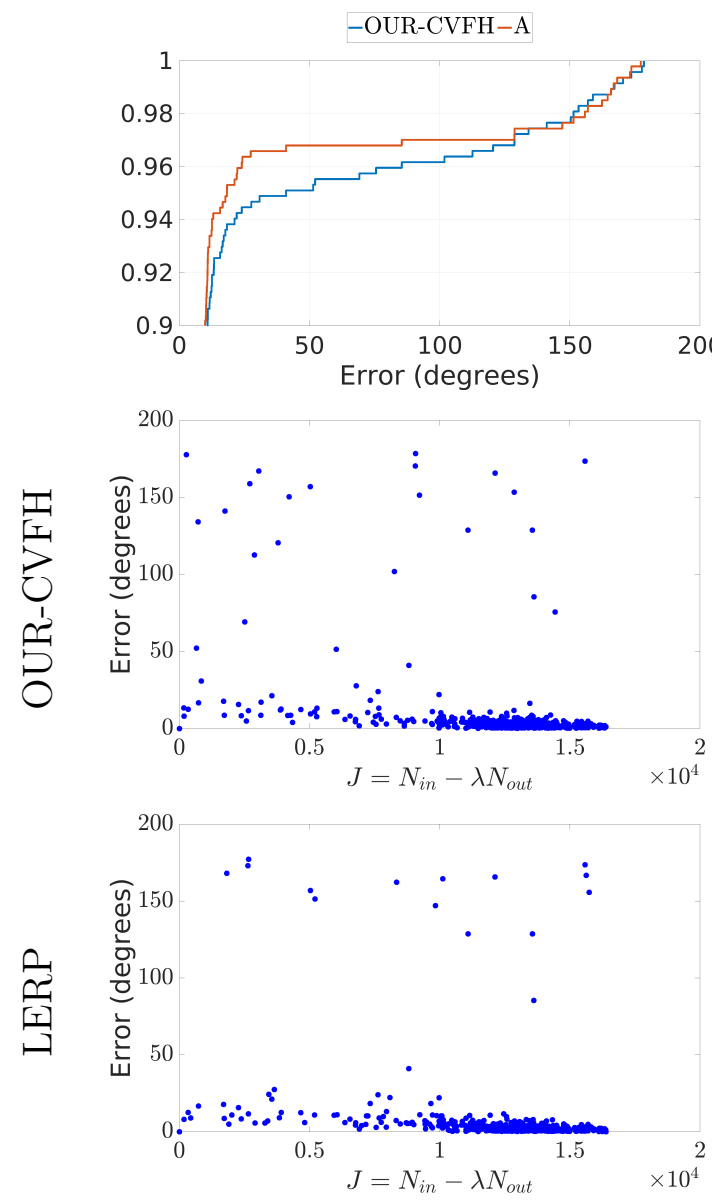

Range Error
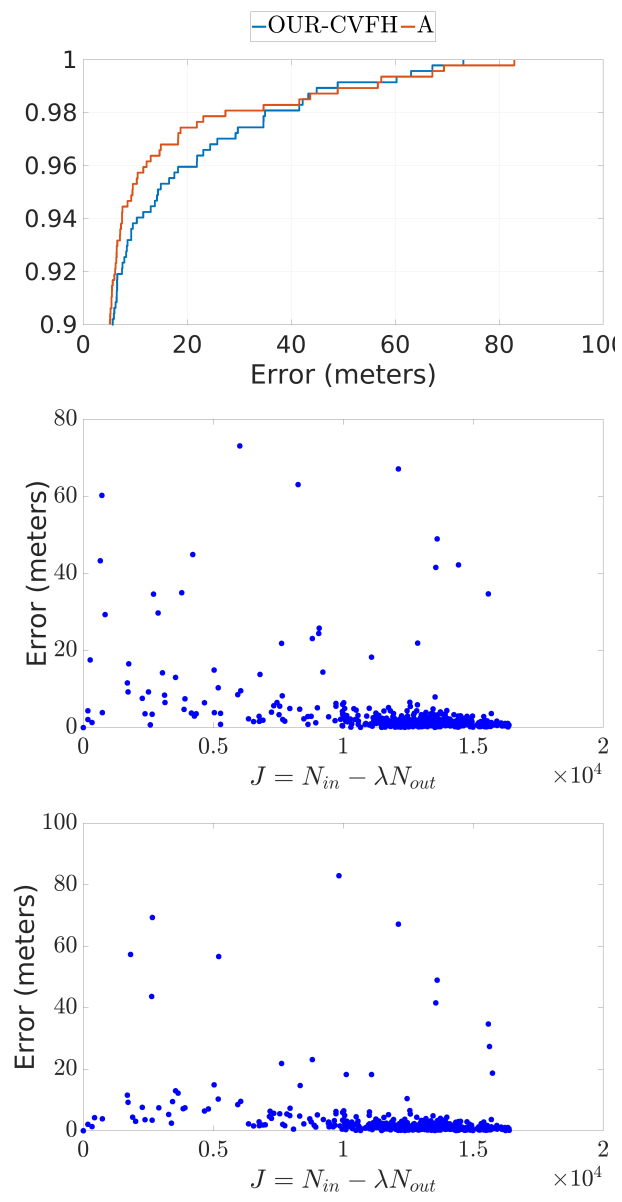

Figure 5.10: Pose estimation errors for 500 run Monte Carlo experiment comparing OUR-CVFH to linear interpolation of angular distribution components. First row shows the box plots of the errors without outliers. Second and third rows show all pose estimation errors as a function of cost metric value. 


\subsubsection{Linear Interpolation}

The OUR-CVFH histogram contains five components, of which four describe angular distributions and one describes the points' spatial distribution. In the original work, only the spatial component (i.e. ISOC [16]) is interpolated to account for various factors such as reference frame orientation uncertainty, partial occlusions, or sensor noise [54]. It has long been the standard of feature histogram construction to interpolate all components across neighboring bins $[74,88,66,68,139,140]$. Our first modification to OUR-CVFH is to linear interpolate (LERP) the four angular distributions $\cos \left(\alpha_{i}\right), \cos \left(\phi_{i}\right), \theta_{i}$, and $\cos \left(\beta_{i}\right)$. This improves the histogram's robustness to variations in vector orientations caused by sensor noise or partial occlusions.

A graphical comparison of bin accumulation is shown in Fig. 5.8 for OUR-CVFH and the proposed LERP. Independent from the number of bins used for a histogram component, each bin is centered around the bin number plus one-half (e.g. 1.5). Suppose the $i$-th element of $\cos \left(\alpha_{i}\right)$ is found to have a floating bin value of 3.8 - it is in the third bin, but closer to the fourth bin than the second. Each bin accumulates the weight of $1-d$ where $d$ is the distance from the floating bin value to the center bin value. For this example, the third bin accumulates a weight of $1-0.3=0.7$, and the fourth bin accumulates a weight of $1-0.7=0.3$. The total amount of weight does not change, just the bin in which it is located.

Our analysis compares the pose estimation errors of OUR-CVFH with and without linear interpolation of the angular distribution components. Box 


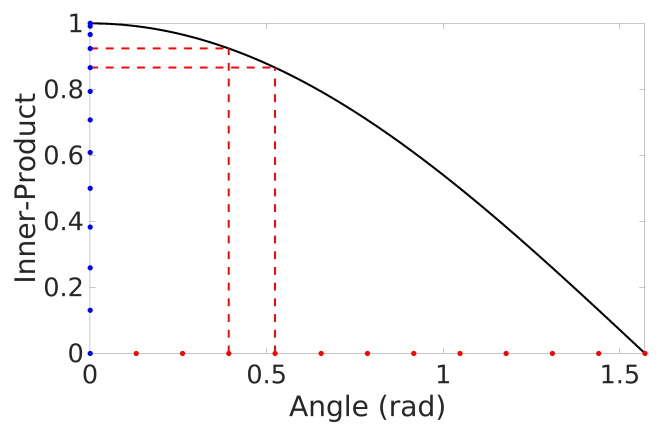

(a) Angular to Inner-Product

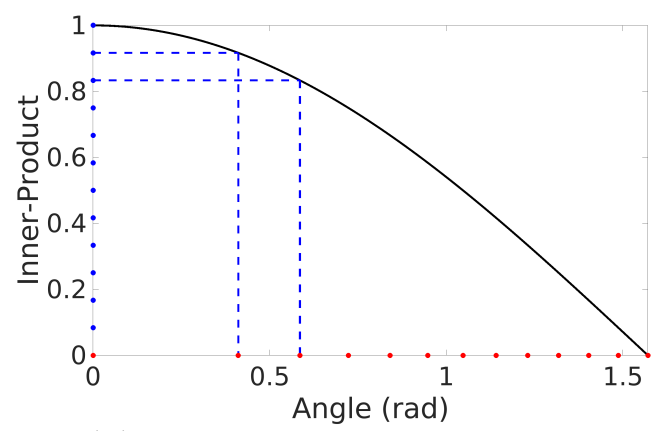

(b) Inner-Product to Angular

Figure 5.11: Spacing between inner-product space and angular space is not equal. (a) Equal spacing in angular space converted through $\cos (\cdot)$ to unequal spacing in inner-product space. (b) Equal spacing in inner-product space is converted through $\cos (\cdot)$ to unequal spacing in angular space.

plots of the errors without outliers as well as estimation errors as a function of cost metric value are shown in Fig. 5.10. Linear interpolation of the angular distribution histogram components offers comparable pose estimation results to OUR-CVFH . Of the 500 runs, OUR-CVFH returns 470 pose estimates, while only 469 pose estimates with the LERP modification.

\subsubsection{Histogram Component Description Space}

There are two description spaces in which features may be described: inner-product space or angular space. These spaces are discretized into equally sized bins for accumulating feature instances. Since the mapping function from one description space to the other is a non-linear trigonometric function, equal spacing in the inner-product space is inequivalent to equal spacing in the angular space. Fig. 5.11 exhibits the differences in mapping between innerproduct and angular spaces. Equally sized angular bins mapped by cosine 

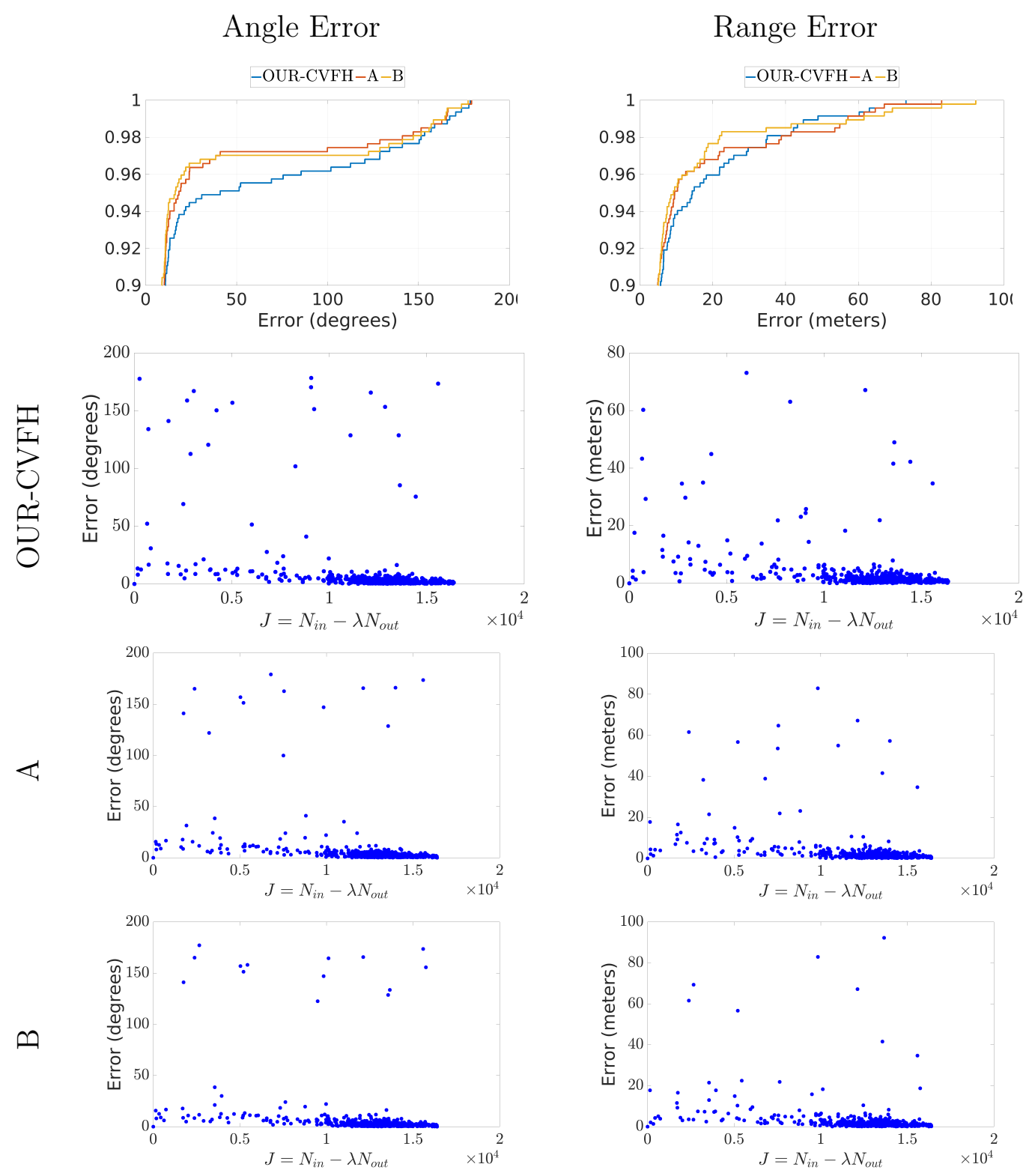

Figure 5.12: Pose estimation errors for 500 run Monte Carlo for varying the description space of $\beta_{i}$. Method $\mathrm{A}$ describes $\beta_{i}$ in angular space, Method $\mathrm{B}$ describes $\beta_{i}$ in angular space and applies LERP to all angular distribution features. The first row shows box plots of the pose estimation errors without outliers. All errors as a function of cost metric value are shown in the second through fourth rows. 
Table 5.2: Statistics for the 500 run Monte Carlo experiment varying the description space of $\beta_{i}$ and linear interpolation to OUR-CVFH . Method A describes $\beta_{i}$ in angular space, Method $\mathrm{B}$ describes $\beta_{i}$ in angular space and applies LERP to all angular distribution features.

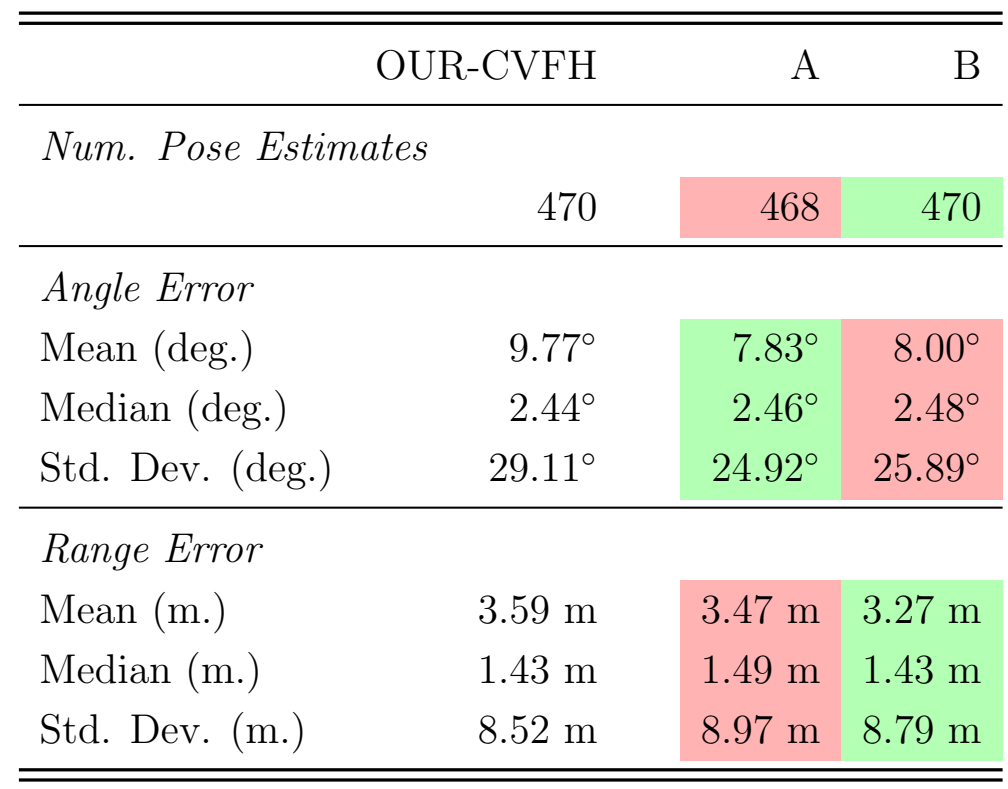

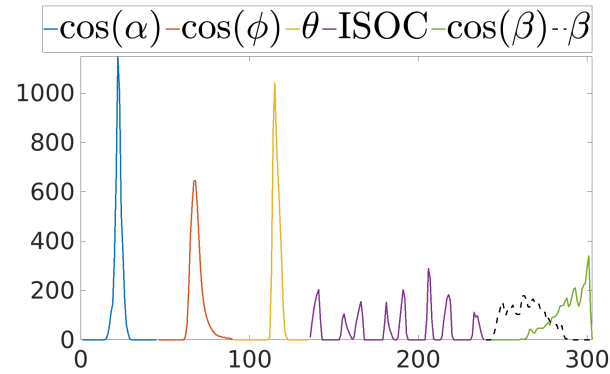

(a) Histogram with $\beta$ in both spaces

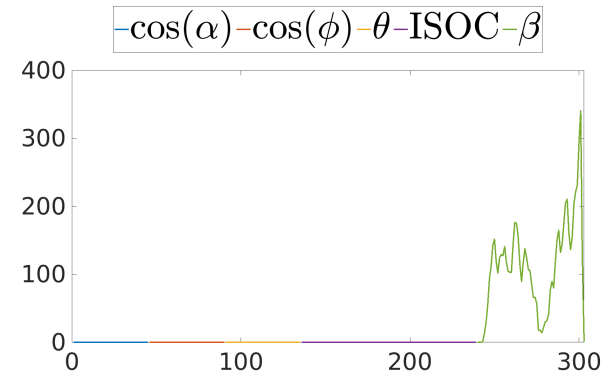

(b) Difference between histograms

Figure 5.13: Visual affects of description space of $\beta_{i}$ on histogram appearance. (a) Histograms shown in colors corresponding to the components (both using LERP). Proposed description of $\beta$ in black dashed line. (b) Absolute difference between histograms color coded by component. Notice change is only with $\beta$. 


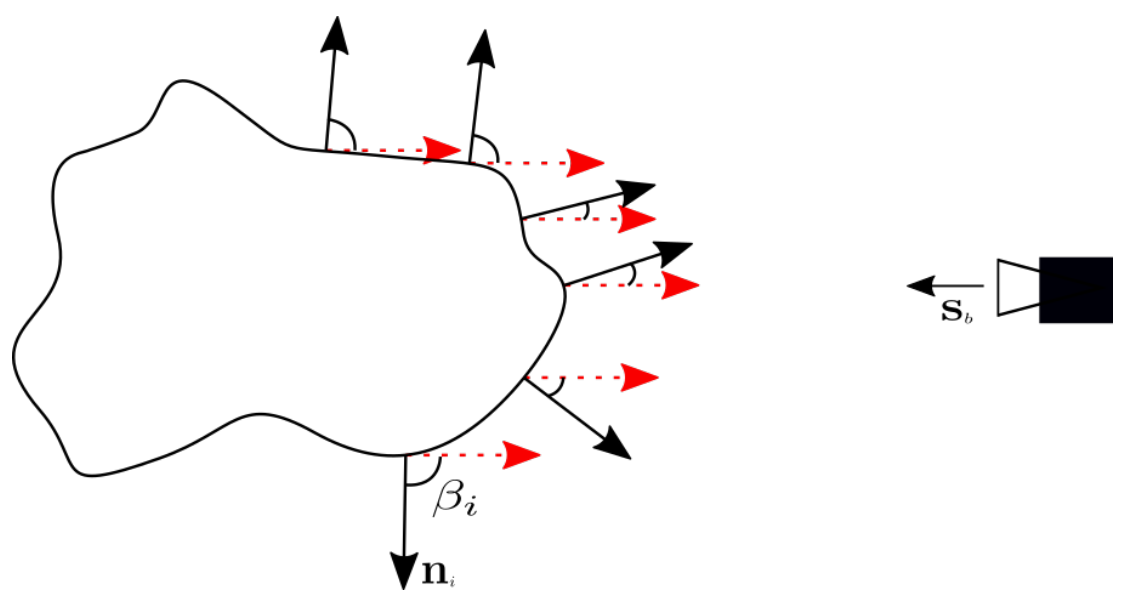

Figure 5.14: A sensor view of an object. Inner product between surface normal vectors $\mathbf{n}_{i}$ and reverse camera boresight $\mathbf{s}_{b}$ (shown in red) create the angle $\beta$. Only small values of $\beta$ are possible, as large values signify that the surface is pointing away from the sensor.

yield large bins around 0 and smaller sized bins around \pm 1 . There is a similar occurrence for mapping from inner-product to angular space. Since description spaces are inequivalent, we must purposefully decide in which space to describe certain features.

For OUR-CVFH, three of the features $-\cos \left(\alpha_{i}\right), \cos \left(\phi_{i}\right)$, and $\cos \left(\beta_{i}\right)-$ exist in inner-product space, while $\theta_{i}$ exist in angular space. Throughout the family of OUR-CVFH publications [79, 78, 54, 77, 76], no comment on which description space these features should exist in is available, nor is reasoning of their selection. Therefore, we perform a though experiment to determine the proper spaces for each of the features. The reader may refer to Fig. 2.3 for additional assistance. Physically, $\alpha_{i}$ and $\phi_{i}$ are generally around $90^{\circ}$. Since their histograms exist in inner-product space, bins are smaller and thus more 
descriptive about $90^{\circ}$ than around $0^{\circ}$ or $180^{\circ}$. We maintain their description in the inner-product space. The angular component $\beta_{i}$ describes the orientation of the sensor with the scene. Oftentimes the sensor is nadir pointing towards objects, then $\beta_{i}$ is generally around $0^{\circ}$. Up to this point, its description has been in the inner-product space where the bins are larger (and thus less descriptive) around $0^{\circ}$ than near $90^{\circ}$. It would be intuitive to change the histogram component $\cos \left(\beta_{i}\right)$ to be described in angular space, yielding

$$
\beta_{i}=\arccos \left(\mathbf{n}_{i}^{\top} \mathbf{v}\right)
$$

Our analysis compares the pose estimation errors of OUR-CVFH when describing $\beta$ in both angular space and inner-product space. Note that we also include the modification from the previous section of linear interpolation across bins for the angular features. Box plots of the errors without outliers as well as estimation errors as a function of cost metric value are shown in Fig. 5.12. Method A describes $\beta_{i}$ in angular space and Method B describes $\beta_{i}$ in angular space with LERP for all angular distribution features. Comparable pose estimation errors are provided by all three methods as shown by the box plots. Notice on the scatter plots for Method B that there are fewer intermediate pose estimation errors - either the errors are small or large, regardless of cost metric value. For the 500 runs, OUR-CVFH yields 470 pose estimations while Method A and B yield 468 and 470 respectively. 


\subsubsection{Pose Estimation Cost Metric}

In OUR-CVFH, the model cluster and corresponding pose estimate that maximizes the cost function of Eq. 2.91 is selected as the best possible match. This cost metric depends on a tuning parameter $\lambda$, which is unset by [54] and set to unity in [16]. Furthermore, if the cost function is negative, then no result is provided. Properly setting $\lambda$ is unclear, as it affects both the quantity and quality of pose estimates. We suggest a different cost metric that does not rely on any tuning parameters. We modify Step 4 from Sec. 2.2.5.5 to use the new cost metric

$$
J=\frac{N_{i n}}{|\mathcal{P}-|}
$$

where $N_{\text {in }}$ is the number of inliers, $\mathcal{P}^{-}$is the model point-cloud produced in training that contains the model cluster being matching and excludes outliers, and $|\cdot|$ returns the set cardinality (i.e. number of points). The best candidate pose estimate is the one that maximizes this cost metric. Using this method, a pose estimate is always provided because $J$ is always bounded by $J \in[0-1]$.

Similar to OUR-CVFH, we also account for occlusions. After ICP, with the model point-cloud in the estimated scene frame, we back-project the model points onto the camera. If the point is not visible on the sensor, then it is removed from consideration before using Eq. 5.13. Inlier, outlier, and occluded points are related through

$$
\left|\mathcal{P}^{-}\right|=|\mathcal{P}|-N_{\text {occlude }}=N_{\text {in }}+N_{\text {out }}
$$



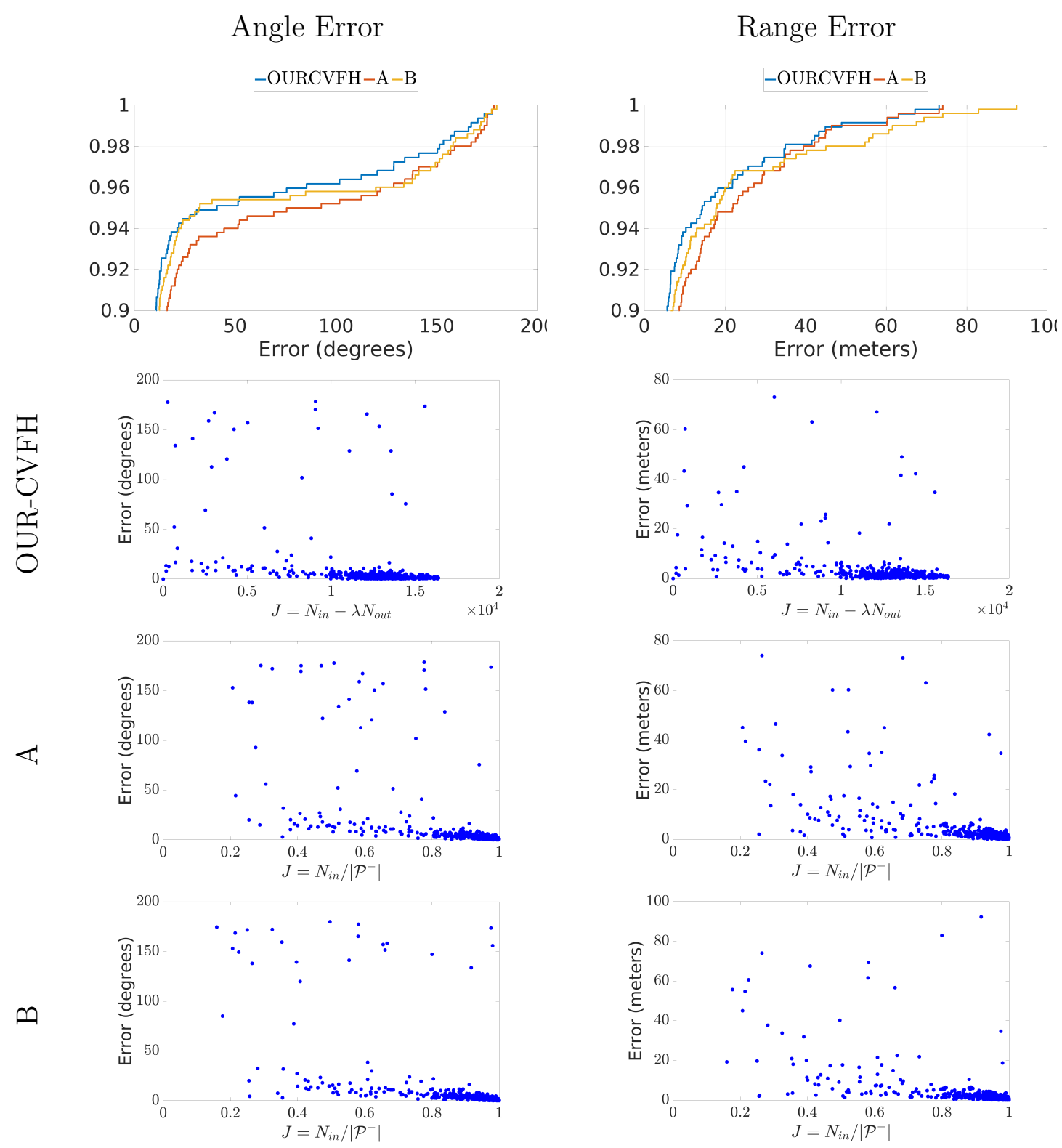

Figure 5.15: Pose estimation errors in CDF and verse cost metric values for various descriptor settings. Method A is OUR-CVFH using the cost metric of Eq. 5.13 and Method B describes $\beta_{i}$ in angular space, uses LERP for angular distribution components, and the cost metric of Eq. 5.13. 
Table 5.3: Statistics for the 500 run Monte Carlo experiment varying the description space of $\beta_{i}$ and linear interpolation to OUR-CVFH . Method A describes $\beta_{i}$ in angular space, Method $\mathrm{B}$ describes $\beta_{i}$ in angular space and applies LERP to all angular distribution features.

\begin{tabular}{|c|c|c|c|}
\hline & -CVFH & $\mathrm{A}$ & $\mathrm{B}$ \\
\hline \multicolumn{4}{|c|}{ Num. Pose Estimates } \\
\hline & 470 & 500 & 500 \\
\hline \multicolumn{4}{|l|}{ Angle Error } \\
\hline Mean (deg.) & $9.77^{\circ}$ & $11.88^{\circ}$ & $10.63^{\circ}$ \\
\hline Median (deg.) & $2.44^{\circ}$ & $2.43^{\circ}$ & $2.48^{\circ}$ \\
\hline Std. Dev. (deg.) & $29.11^{\circ}$ & $33.00^{\circ}$ & $31.49^{\circ}$ \\
\hline \multicolumn{4}{|l|}{ Range Error } \\
\hline Mean (m.) & $3.59 \mathrm{~m}$ & $4.18 \mathrm{~m}$ & $4.13 \mathrm{~m}$ \\
\hline Median (m.) & $1.43 \mathrm{~m}$ & $1.40 \mathrm{~m}$ & $1.40 \mathrm{~m}$ \\
\hline Std. Dev. (m.) & $8.52 \mathrm{~m}$ & $9.41 \mathrm{~m}$ & $10.58 \mathrm{~m}$ \\
\hline
\end{tabular}


where $N_{\text {out }}$ is the number of outlier points and $N_{\text {occlude }}$ is the number of occluded points.

Our analysis compares the pose estimation of OUR-CVFH with the two cost metrics of Eq. 2.91 and Eq. 5.13. We also include the previous modifications of (1) LERP of the angular distribution components and (2) describing $\beta_{i}$ in the angular space. Box plots of the pose estimation errors without outliers are shown in Fig. 5.15. Method A is OUR-CVFH using the cost metric of Eq. 5.13 and Method B describes $\beta_{i}$ in angular space, uses LERP for angular distribution components, and the cost metric of Eq. 5.13.

Fig. 5.15 shows CDF plots of OUR-CVFH and Methods A and B. Tab. 5.3 shows the statistics of the two methods compared to OUR-CVFH . The distribution of all pose estimates as a function of cost metric value are shown in Fig. 5.15. In this scenario, OUR-CVFH outperforms Methods A and $\mathrm{B}$. The reason is that OUR-CVFH only returns 470 pose estimates while Methods A and B both return a pose estimate for all 500 Monte Carlo runs. While this modification temporarily worsens our methods, it provides a more intuitive situation of limiting poor pose estimates.

If an accurate descriptor match is not found, then it is desired that a pose estimate not be returned. This would prevent poor pose estimates being ingested into a navigation filter. The OUR-CVFH cost metric of the first row is unbounded and has a wide range, thus, it would difficult to set a threshold for which to ignore estimates. OUR-CVFH does not return an estimate in instances when the cost metric of Eq. 2.91 is negative, but selecting a value 
for $\lambda$ is ill-defined. Instead, it would be more intuitive to set a minimum threshold $t_{J}$ for which Eq. 5.13 would be required to satisfy before accepting the pose estimate. We do not set the threshold $t_{J}$ at this time because the SOUR-CVFH descriptor is still in development. Its value will be set in Sec. 5.3.

\subsubsection{Histogram Normalization}

The OUR-CVFH histogram consists of five individual components: $\alpha$, $\phi, \theta$, ISOC, and $\beta$. Four of these components $(\alpha, \phi, \theta$, and $\beta)$ are formed by accumulating angular distributions into discretized bins, while the fifth (ISOC) is for the spatial distribution. For each component, the summation of their bins equals the number of points in the cluster $m_{\mathcal{K}}$. We normalize each histogram component individually such that the summation of their bins totals unity. In this way, the histogram is representative of the cluster shape probability. Viewing the histogram as a probability distribution offers an opportunity to use statistical inference and compare various distance metrics availably only to normalized distributions. Additionally, each histogram component may be utilized individually as a histogram descriptor. Because each component is normalized individually, the total summation of each histogram is equal to the total number of components - in our case 5.

The affect of histogram normalization on pose estimation errors is not investigated here for multiple reasons. First, up to this point, we still use the distance function of Eq. 2.88 which includes a " $1+$ " on the numerator and denominator. It would have undue influence on a normalized histogram. 


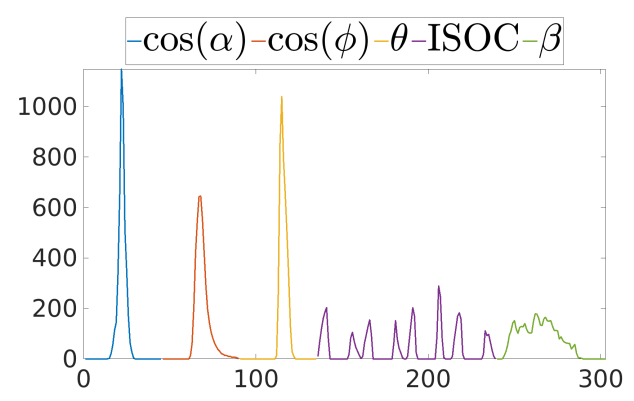

(a) Unnormalized

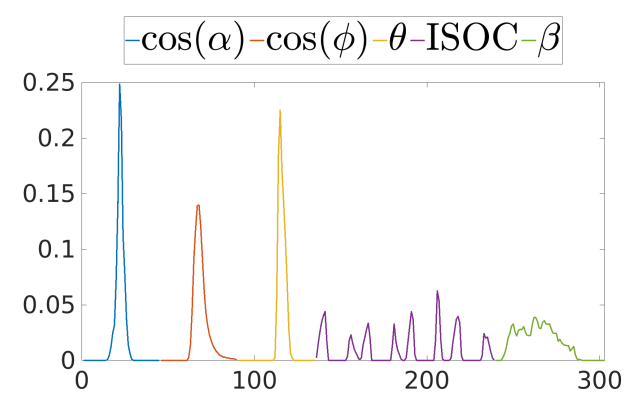

(b) Normalized

Figure 5.16: Normalization of histogram individually by component.

Second, a normalized histogram is considered a probability distribution and other distance metrics are available. Third, by normalizing the histogram, all information regarding cluster size is eliminated. An upcoming section will provide a method of matching cluster scale.

\subsubsection{Distance Metric Selection}

Let's reevaluate the distance metric for matching histograms. Given a normalized histogram such that the sum of all the components is unity, the histogram may be considered a discrete probability distribution. With this comes the opportunity to use distance metrics reserved for calculating the divergence between probability distributions. We investigate four distance metrics to use with our SOUR-CVFH descriptor, namely the Jaccard distance, KullbackLeibler divergence, Jensen-Shannon divergence, and a symmetric Chi-Square test.

The Jaccard index tests the similarity between two sets of data. We are interested in the dissimilarity between two histograms $\mathcal{H}$, which is it's 
compliment. One disadvantage to the Jaccard metric is that it is sensitive to data occlusions, which is probably in the case of measured LIDAR data. The Jaccard (JC) distance is

$$
\mathrm{d}_{\mathrm{JC}}(\widetilde{\mathcal{H}}, \mathcal{H})=1-\frac{\sum_{i=1}^{303} \min \left(\widetilde{\mathcal{H}}_{i}, \mathcal{H}_{i}\right)}{\sum_{i=1}^{303} \max \left(\widetilde{\mathcal{H}}_{i}, \mathcal{H}_{i}\right)}
$$

The Kullback-Leibler (KL) divergence is the measure of the difference between to probability distributions. The histogram must be normalized in order to use this metric. Unfortunately, KL is an asymmetric measure depending on which histogram $\mathcal{H}$ is considered the primary and secondary. Because of this asymmetry, we calculate the KL divergence in both directions and take the maximum result. The KL divergence is $[141,76]$

$$
\mathrm{d}_{\mathrm{KL}}(\widetilde{\mathcal{H}} \mid \mathcal{H})=\sum_{i=1}^{303} \widetilde{\mathcal{H}}_{i} \ln \left(\frac{\widetilde{\mathcal{H}}_{i}}{\mathcal{H}_{i}}\right)
$$

The Jensen-Shannon (JS) divergence is a special case of the KL divergence. The JS divergence is one-half times the sum of the KL divergences calculated in both directions. Because it is an average of the the KL divergence of both directions, it is a symmetric measure and written as

$$
\mathrm{d}_{\mathrm{JS}}(\widetilde{\mathcal{H}}, \mathcal{H})=\frac{1}{2} \mathrm{~d}_{\mathrm{KL}}(\widetilde{\mathcal{H}} \mid \mathcal{H})+\frac{1}{2} \mathrm{~d}_{\mathrm{KL}}(\mathcal{H} \mid \widetilde{\mathcal{H}})
$$

The JS distance is calculated as the square-root of Eq. 5.17. We analyze the divergence because is avoids the additional square-root step. Either metric would provide identical results. 
Pearson's Chi-Squared test is a statistical measure of the difference between two data sets. The Chi-Squared test is asymmetric, but we use a symmetric version of the Chi-Squared (CS) given as [141, 142]

$$
\mathrm{d}_{\chi^{2}}(\widetilde{\mathcal{H}}, \mathcal{H})=\sum_{i=1}^{303} \frac{\left(\widetilde{\mathcal{H}}_{i}-\mathcal{H}_{i}\right)^{2}}{\widetilde{\mathcal{H}}_{i}+\mathcal{H}_{i}}
$$

The Chi-Squared distance is the square-root of Eq. 5.18. We analyze the divergence, but either would provide identical results.

The distance metric used by OUR-CVFH in Eq. 2.88 is most similar the Jaccard dissimilarity of Eq. 5.15 with the notably difference of a "1+" in the denominator and numerator. Since the OUR-CVFH descriptor is unnormalized and the histogram will contain values must large than 1 , the " $1+$ " has little overall affect on the distance calculation. Contrastingly, for our normalized SOUR-CVFH descriptor, the "1+" would have an extreme influence on our distance calculation. For this reason, we do not use the OUR-CVFH distance metric, but do consider the similar Jaccard dissimilarity.

Our analysis compares the pose estimation results of OUR-CVFH to various distance metrics. All other modifications up to this point are also included when testing the new metrics: (1) LERP of angular distributions, (2) describing $\beta_{i}$ in the angular space, (3) new cost metric of Eq. 5.13, and (4) histogram normalization. Box plots of the pose estimation errors without outliers are shown in Fig. 5.17. The JC and CS metrics both provide pose estimation errors comparable to OUR-CVFH, while the KL and JS metrics yield larger errors. 


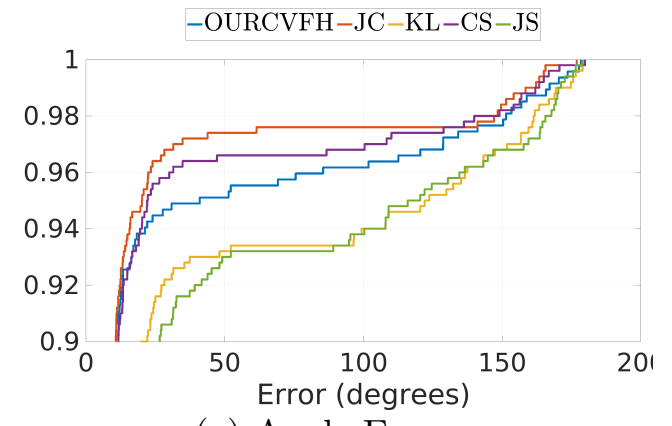

(a) Angle Error

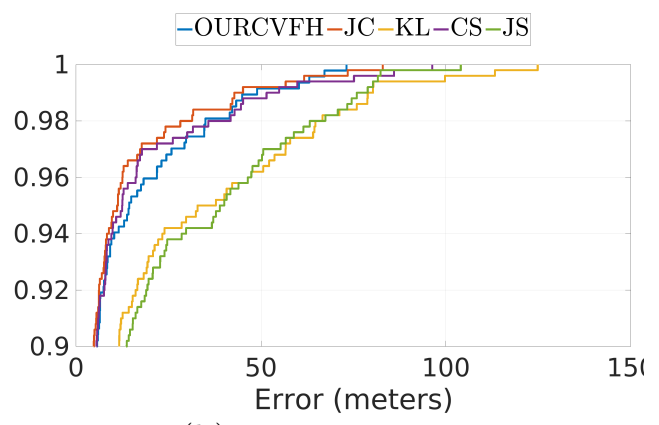

(b) Range Error

Figure 5.17: Pose estimation errors without outliers for 500 run Monte Carlo varying the distance metric. Distances used are Jaccard (JC), Kullback-Leibler (KL), Chi-Squared (CS), and Jensen-Shannon (JS). Results are compared to OUR-CVFH .

Table 5.4: Statistics for the 500 run Monte Carlo experiment varying distance metric.

\begin{tabular}{llllll}
\hline \hline & OUR-CVFH & JC & KL & CS & JS \\
\hline \multicolumn{2}{c}{ Num. Pose Estimates } & & & & \\
& 470 & 500 & 500 & 500 & 500 \\
\hline Angle Error & & & & & \\
Mean (deg.) & $9.78^{\circ}$ & $7.29^{\circ}$ & $14.02^{\circ}$ & $8.39^{\circ}$ & $14.52^{\circ}$ \\
Median (deg.) & $2.44^{\circ}$ & $1.67^{\circ}$ & $2.55^{\circ}$ & $1.90^{\circ}$ & $2.57^{\circ}$ \\
Std. Dev. (deg.) & $29.12^{\circ}$ & $24.21^{\circ}$ & $35.75^{\circ}$ & $26.31^{\circ}$ & $36.04^{\circ}$ \\
\hline Range Error & & & & & \\
Mean (m.) & $3.59 \mathrm{~m}$ & $2.97 \mathrm{~m}$ & $6.35 \mathrm{~m}$ & $3.41 \mathrm{~m}$ & $6.38 \mathrm{~m}$ \\
Median (m.) & $1.43 \mathrm{~m}$ & $1.02 \mathrm{~m}$ & $1.69 \mathrm{~m}$ & $1.11 \mathrm{~m}$ & $1.60 \mathrm{~m}$ \\
Std. Dev. (m.) & $8.52 \mathrm{~m}$ & $7.92 \mathrm{~m}$ & $15.20 \mathrm{~m}$ & $9.30 \mathrm{~m}$ & $14.28 \mathrm{~m}$ \\
\hline \hline
\end{tabular}



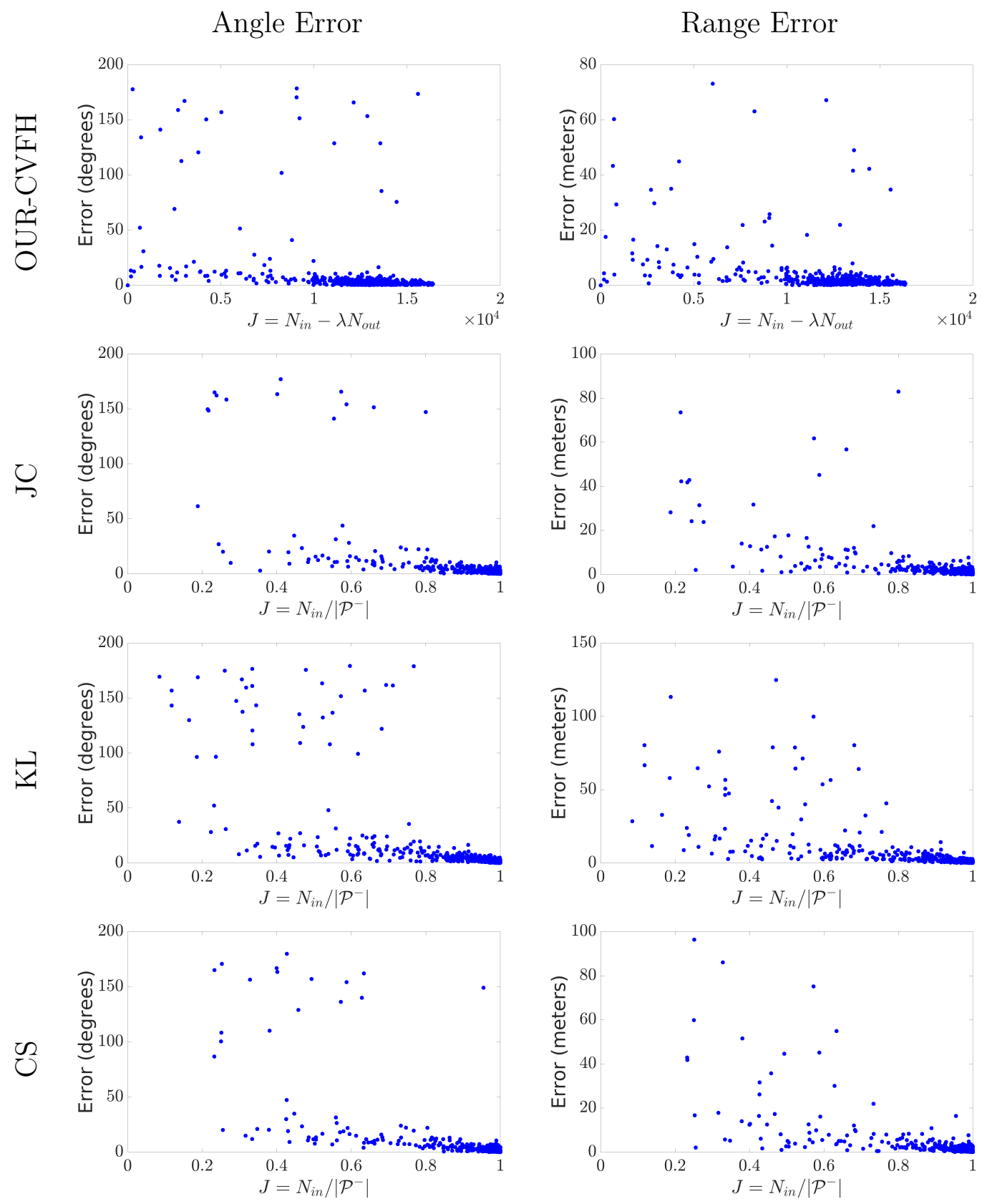

Figure 5.18: All pose estimation errors as a function of cost metric value for 500 run Monte Carlo varying the distance metric. Distances used are Jaccard (JC), Kullback-Leibler (KL), Chi-Squared (CS), and Jensen-Shannon (JS). Compare to the OUR-CVFH results in first row of Fig. 5.10. 
Angle Error

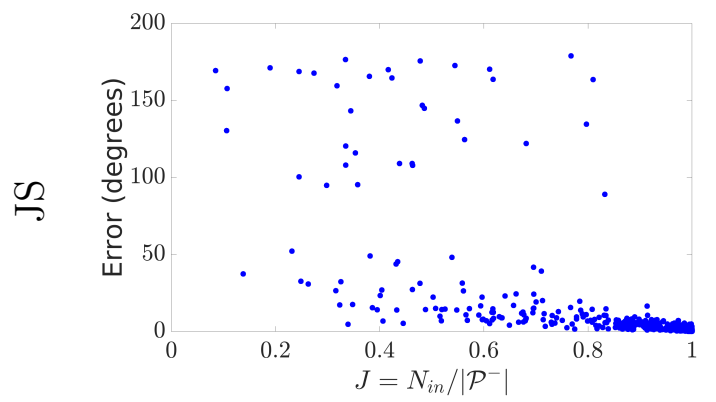

Range Error

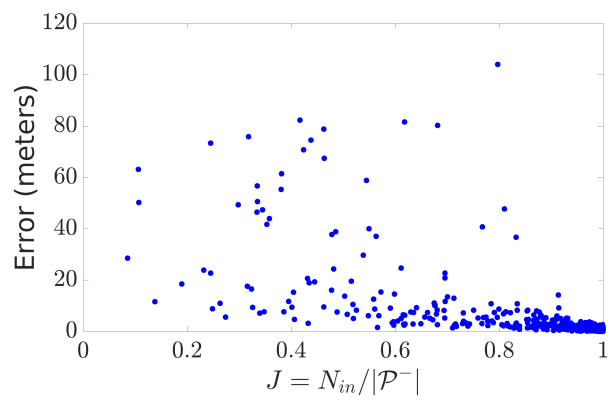

Figure 5.18: Continued.

All pose estimation errors as a function of cost metric value are shown in Fig. 5.18. OUR-CVFH results are shown in Fig. 5.10. Recall that the cost metric of Eq. 5.13 yields a pose estimate in every instance, while OURCVFH occasionally omits estimates. For angular errors on both JC and CS metrics, there is a clear distinction between good and poor estimates. This separation allows for easy residual edit checks in a navigation filter for rejection of poor estimates.

When we normalized the histogram, we lost information regarding the cluster size - namely the quantity of points in the cluster. The height of the OUR-CVFH histogram is representative of the cluster size, but only in the sense of the quantity of points, not necessarily the cluster scale (see Sec. 5.1.5). The OUR-CVFH histogram height is more representative of the LIDAR resolution. In the absence of a scale factor, the OUR-CVFH histogram height is a decent substitute. The next section will introduce a scale factor to our descriptor. 
Since both JC and CS metrics performed similarly, and we still have yet to include a scale factor, we do not yet select a distance metric. We will analyze both the JC and CS metrics in the next section when introducing scale.

\subsubsection{Cluster Scale as an Identifier}

Object scale is an important attribute to use in matching and identification. An advantage of using point-clouds over 2D images for object recognition is that scale is explicitly inherent to the range measurements. Including the scale in the recognition framework will (1) improve pose estimation by only considering clusters of similar scale and (2) decrease the runtime by limiting the number of histograms to compare. This section presents a method of analyzing a cluster's scale.

Previously, OUR-CVFH considered the histogram's height as representative of the object scale. The thought experiment in Sec. 5.1.5 explains how two clusters of different scale, but identical number of points, may results in the same histogram. Instead, the height of the OUR-CVFH histogram is most reflective of the sensor resolution. If two sensors of resolution $r_{1}$ and $r_{2}$ where $r_{2}>r_{1}$ were to measure the same scene, the same clusters would be identified, but the histograms would have different heights. Again, the OUR-CVFH descriptor fails to explicitly recognize scale. Since the current histogram is normalized, a different approach must be taken to explicitly incorporate scale. 
Recall the OUR-CVFH scatter matrix M presented in Eq. 2.63. Defining the SGURF requires taking the eigen-decomposition of $\mathbf{M}$ and disambiguating the axes using the whole point-cloud. Eigen-decomposition returns (1) eigenvectors defining the principle axes and (2) eigenvalues describing the characteristic length along the axes. Only the eigenvectors are utilized in constructing the SGURF and OUR-CVFH disregards the eigenvalues. We use these eigenvalues to describe the cluster scale.

Suppose the scatter matrix of Eq.2.63 has the eigen-decomposition

$$
\mathbf{M e}_{i}=\lambda_{i} \mathbf{e}_{i}
$$

where $\mathbf{e}_{i}$ is an eigenvector and $\lambda_{i}$ is an eigenvalue of $\mathbf{M}$. Generating the SGURF reorganizes the order of the eigenvectors according to the disambiguations scheme. Tracking the corresponding eigenvalues will provide the scale along those axes. The scales along the axes are found as

$$
\sigma_{i}=3 \sqrt{\lambda_{i}} \quad i=1, \ldots, 3
$$

where $\sigma_{i}$ corresponds to the $i$-th axes and the support region is 3 . The clustering method presented in Section 2.2.5.1 prioritizes nearly planar clusters that are considered to lie in 2D. A support window of 3 offers $98.8 \%$ coverage in 2D.

Each cluster is associated with three scales, one for each axis. Since the clustering algorithm favors planar clusters, the out-of-plane axis (i.e. $z$-axis) has a small eigenvalue, and it is similar for nearly all clusters. Comparing 

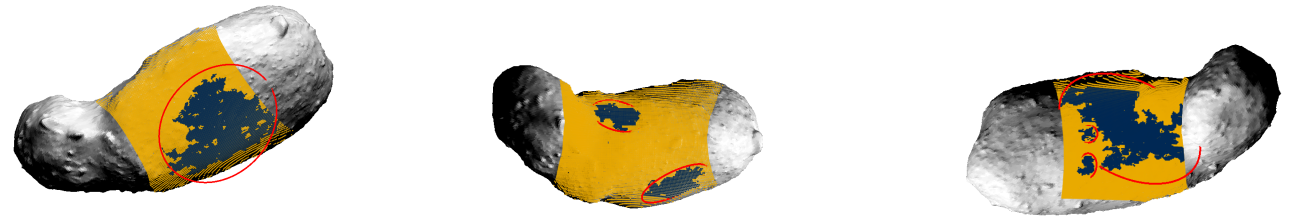

Figure 5.19: Model Itokawa with scene shown in yellow, clusters shown in blue, and the cluster's bounding circle defined by the scale shown in red. Only 2D circles are shown for clarity.

scales of the $z$-axis is mostly uninformative. The other in-plane axes offer the most information on cluster scale.

When matching SOUR-CVFH histograms, the scale components are first compared to ensure that the clusters are of similar size. If the scales are not similar, then the histograms are not matched. This efficient approach performs a single value comparison that limits the quantity of histogram matches. It reduces the computational burden of the matching algorithm and eliminates the potential of matching histograms of extremely different sized clusters. Comparing the cluster scale is as follows:

1. Compare the scales of every scene cluster $\mathcal{C}_{k} \in \mathcal{S}$, to the scales of every model cluster $\mathcal{C}_{k} \mathcal{M}$ through the scale ratios as

$$
\mathrm{r}_{\sigma, j}=\frac{\min \left(\sigma_{\widetilde{\delta_{, j}}}, \sigma_{\mathcal{M}, j}\right)}{\max \left(\sigma_{\widetilde{\S, j}}, \sigma_{\mathcal{M}, j}\right)} \quad j=1,2
$$

where he scale ratios $\mathbf{r}_{\sigma} \in[0-1]$, where $\mathbf{r}_{\sigma}=0$ is no similarity and $\mathbf{r}_{\sigma}=1$ is identical values. 
2. If the scale ratios are larger than a threshold $t_{r_{\sigma}}$, then the clusters are of similar scale, and histogram matching may proceed.

The $x$ - and $y$-axes of the SGURF are selected as the scatter matrix eigenvectors which have corresponding eigenvalues. Since corresponding axes between two SGURFs are matched, we also match the corresponding axes scales. This allows the most direct comparison of scale similarity. Other options considered were comparing (1) the minimum and maximum scales or (2) the cluster area. The first is inaccurate because it could match an $x$-axis of one SGURF to a $y$-axis of another SGURF. The second could yield false positives when comparing long narrow clusters to small circular clusters with similar areas.

Two questions remain regarding comparing the scales between a scene and model cluster. First, what should be the threshold $t_{\mathrm{r}}$ ? Second, Should both $r_{\sigma, 1}$ and $r_{\sigma, 2}$ be larger than this threshold, or is only one ratio greater than the threshold sufficient?

A Monte Carlo analysis is performed to test pose estimation accuracy for a variety of values for $t_{\mathrm{r}} \in[0.7,0.8,0.9,0.95]$. We compare both distance metrics of JC and CS, and contrast the results with OUR-CVFH . Recall the modifications that we've introduced: (1) used LERP on all angular distribution features, (2) describe $\beta_{i}$ in angular space, (3) introduced a new cost metric of Eq. 5.13, (4) normalized each component of the histogram, (5) selected a new distance metric, and now (6) comparing scene and model clusters' scales to 
eliminate improbable matches. We analyze separately the effects of requiring either one or both scale ratios to meet the scale threshold.

Pose estimation errors without outliers of the Monte Carlo experiment are shown for JC metric in Fig. 5.20 and for CS metric in Fig. 5.23. The left columns are when only one axis scale ratio is required to meet the threshold, while the right columns are when both axes scale ratios are required to meet the threshold. Requiring only one axis scale to meet the threshold $t_{\mathrm{r}_{\sigma}}$ offers comparable pose estimation errors as OUR-CVFH . Noticeable improvement occurs when both axes scale ratios are required to meet the threshold (right columns). For both JC and CS, the smallest errors occur when $t_{\mathrm{r}_{\sigma}}=0.9$ and both axes scale ratios satisfy the threshold. An upper limit to this threshold does exist, as is evident by the larger pose estimation errors when $t_{\mathrm{r}_{\sigma}}=0.95$. Our objective is to set the scale ratio threshold $t_{\mathrm{r}_{\sigma}}$ as high as possible to eliminate improbably histogram matches and reduce pose estimation errors. We utilize scale comparisons as a first step to eliminate unnecessary matching of histograms for clusters of different sizes. Also recall that OUR-CVFH provides only 470 of 500 pose estimates, while our method yields all 500 pose estimates due to the cost metric of Eq. 5.13.

One of the most significant improvements resulting from scale comparisons is the reduction in the number of histogram matches. These results are shown on a log-scale in the bottom rows of Fig. 5.20 for the JC metric and in Fig. 5.23 for the CS metric. When $t_{\mathrm{r}_{\sigma}}=0.9$, the number of histogram matches is reduced by two orders of magnitude with this train- 
Single Scale Ratio
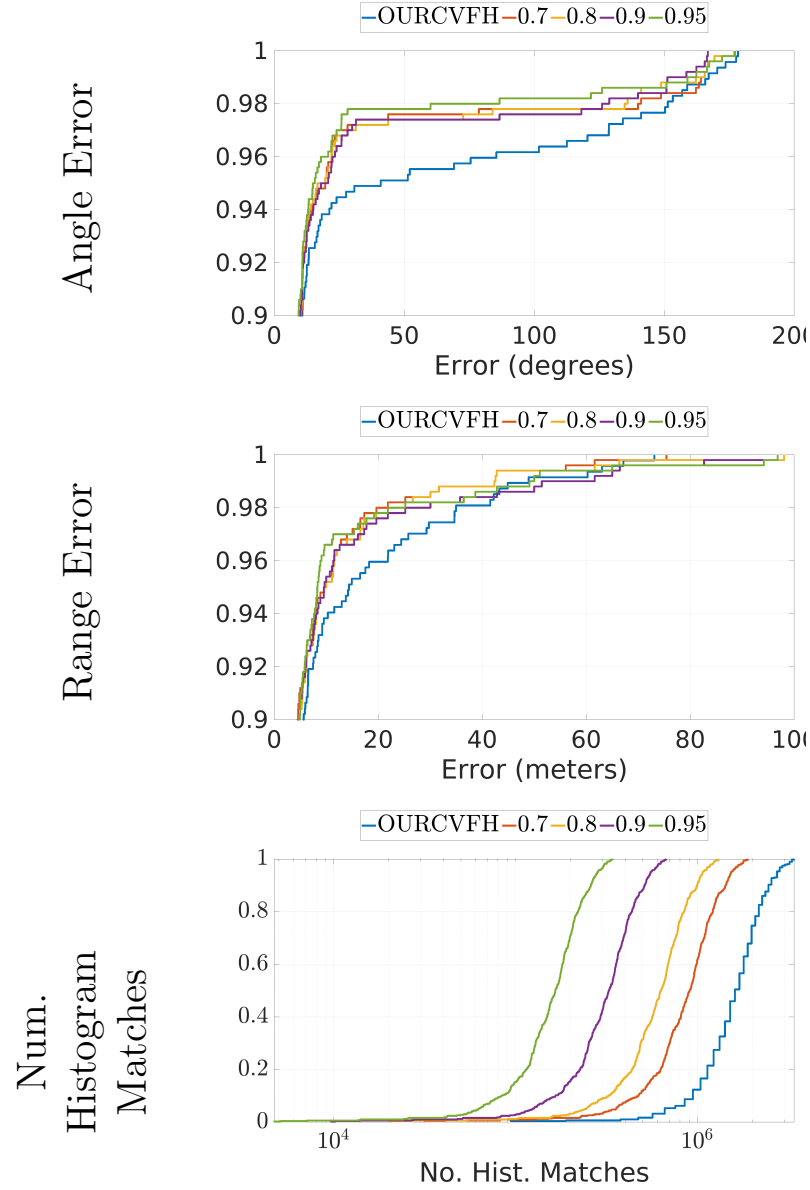

Both Scale Ratio
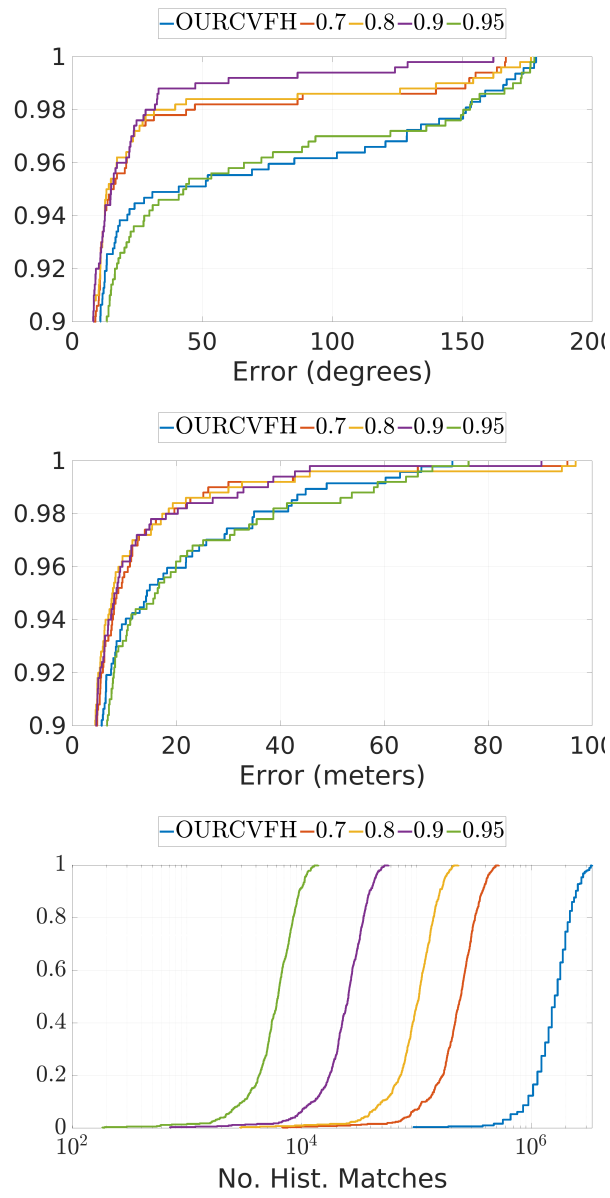

Figure 5.20: Pose estimation errors for 500 run Monte Carlo experiment for various scale thresholds using JC distance. Left column is when one axis scale ratio is greater than $t_{r_{\sigma}}$. Right column is when both scale ratios are greater than $t_{r_{\sigma}}$. Top row is the angular error in degrees, middle row is the range error in meters, bottom row is the number of histogram matches performed. 
Table 5.5: Statistics for the 500 run Monte Carlo experiment scale ratio threshold using distance metric JC.

\begin{tabular}{|c|c|c|c|c|c|}
\hline \multirow{2}{*}{\multicolumn{2}{|c|}{ OUR }} & \multicolumn{4}{|c|}{ one ratio } \\
\hline & & $t_{\mathrm{r}_{\sigma}}=0.7$ & $t_{\mathrm{r}_{\sigma}}=0.8$ & $t_{\mathrm{r}_{\sigma}}=0.9$ & $t_{\mathrm{r}_{\sigma}}=0.95$ \\
\hline \multicolumn{6}{|c|}{ Num. Pose Estimates } \\
\hline & 470 & 500 & 500 & 500 & 500 \\
\hline \multicolumn{6}{|l|}{ Angle Error } \\
\hline Mean (deg.) & $9.78^{\circ}$ & $7.02^{\circ}$ & $6.98^{\circ}$ & $6.96^{\circ}$ & $6.33^{\circ}$ \\
\hline Median (deg.) & $2.44^{\circ}$ & $1.67^{\circ}$ & $1.66^{\circ}$ & $1.64^{\circ}$ & $1.70^{\circ}$ \\
\hline Std. Dev. (deg.) & $29.12^{\circ}$ & $23.82^{\circ}$ & $23.34^{\circ}$ & $23.18^{\circ}$ & $21.35^{\circ}$ \\
\hline \multicolumn{6}{|l|}{ Range Error } \\
\hline Mean (m.) & $3.59 \mathrm{~m}$ & $2.67 \mathrm{~m}$ & $2.80 \mathrm{~m}$ & $3.06 \mathrm{~m}$ & $2.93 \mathrm{~m}$ \\
\hline Median (m.) & $1.43 \mathrm{~m}$ & $1.00 \mathrm{~m}$ & $1.01 \mathrm{~m}$ & $1.01 \mathrm{~m}$ & $1.07 \mathrm{~m}$ \\
\hline Std. Dev. (m.) & $8.52 \mathrm{~m}$ & $6.73 \mathrm{~m}$ & $7.52 \mathrm{~m}$ & $8.86 \mathrm{~m}$ & $8.48 \mathrm{~m}$ \\
\hline \multicolumn{6}{|c|}{ Num. Hist. Matches } \\
\hline Mean (m.) & $1.67 e^{6}$ & $9.12 e^{5}$ & $6.36 e^{5}$ & $3.29 e^{5}$ & $1.67 e^{9}$ \\
\hline Median (m.) & $1.60 e^{6}$ & $9.05 e^{5}$ & $6.30 e^{5}$ & $3.25 e^{5}$ & $1.65 e^{5}$ \\
\hline \multirow[t]{3}{*}{ Std. Dev. (m.) } & $6.10 e^{5}$ & $3.59 e^{5}$ & $2.51 e^{5}$ & $1.29 e^{5}$ & $6.56 e^{4}$ \\
\hline & & \multicolumn{4}{|c|}{ two ratios } \\
\hline & OUR-CVFH & $t_{\mathrm{r}_{\sigma}}=0.7$ & $t_{\mathrm{r}_{\sigma}}=0.8$ & $t_{\mathrm{r}_{\sigma}}=0.9$ & $t_{\mathrm{r}_{\sigma}}=0.95$ \\
\hline Angle Error & & & & & \\
\hline Mean (deg.) & $9.78^{\circ}$ & $5.86^{\circ}$ & $5.61^{\circ}$ & $4.46^{\circ}$ & $9.53^{\circ}$ \\
\hline Median (deg.) & $2.44^{\circ}$ & $1.63^{\circ}$ & $1.63^{\circ}$ & $1.63^{\circ}$ & $1.72^{\circ}$ \\
\hline Std. Dev. (deg.) & $29.12^{\circ}$ & $19.45^{\circ}$ & $19.14^{\circ}$ & $12.61^{\circ}$ & $28.52^{\circ}$ \\
\hline \multicolumn{6}{|l|}{ Range Error } \\
\hline Mean (m.) & $3.59 \mathrm{~m}$ & $2.56 \mathrm{~m}$ & $2.55 \mathrm{~m}$ & $2.49 \mathrm{~m}$ & $3.72 \mathrm{~m}$ \\
\hline Median (m.) & $1.43 \mathrm{~m}$ & $1.01 \mathrm{~m}$ & $1.00 \mathrm{~m}$ & $1.00 \mathrm{~m}$ & $1.11 \mathrm{~m}$ \\
\hline Std. Dev. (m.) & $8.52 \mathrm{~m}$ & $6.63 \mathrm{~m}$ & $7.33 \mathrm{~m}$ & $6.30 \mathrm{~m}$ & $9.19 \mathrm{~m}$ \\
\hline \multicolumn{6}{|c|}{ Num. Hist. Matches } \\
\hline Mean (m.) & $1.67 e^{6}$ & $2.43 e^{5}$ & $1.06 e^{5}$ & $2.58 e^{4}$ & $6.31 e^{3}$ \\
\hline Median (m.) & $1.60 e^{6}$ & $2.43 e^{5}$ & $1.05 e^{5}$ & $2.55 e^{4}$ & $6.22 e^{3}$ \\
\hline Std. Dev. (m.) & $6.10 e^{5}$ & $9.67 e^{4}$ & $4.16 e^{4}$ & $1.03 e^{4}$ & $2.52 e^{3}$ \\
\hline
\end{tabular}



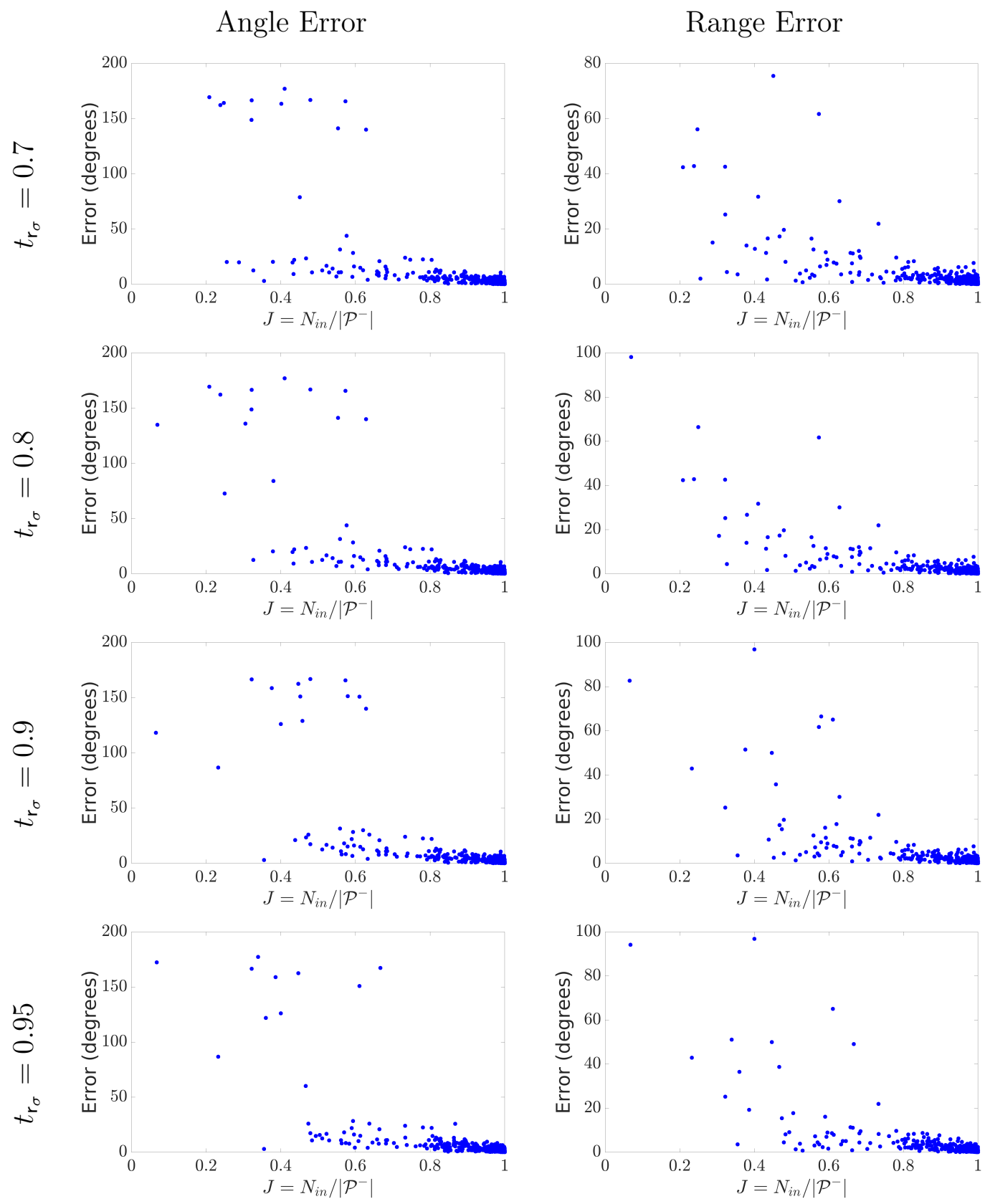

Figure 5.21: All pose estimation errors as a function of cost metric value for 500 run Monte Carlo varying scale threshold $t_{\mathrm{r}_{\sigma}}$ using distance metric JC and requiring one axis scale ratio to meet threshold. 

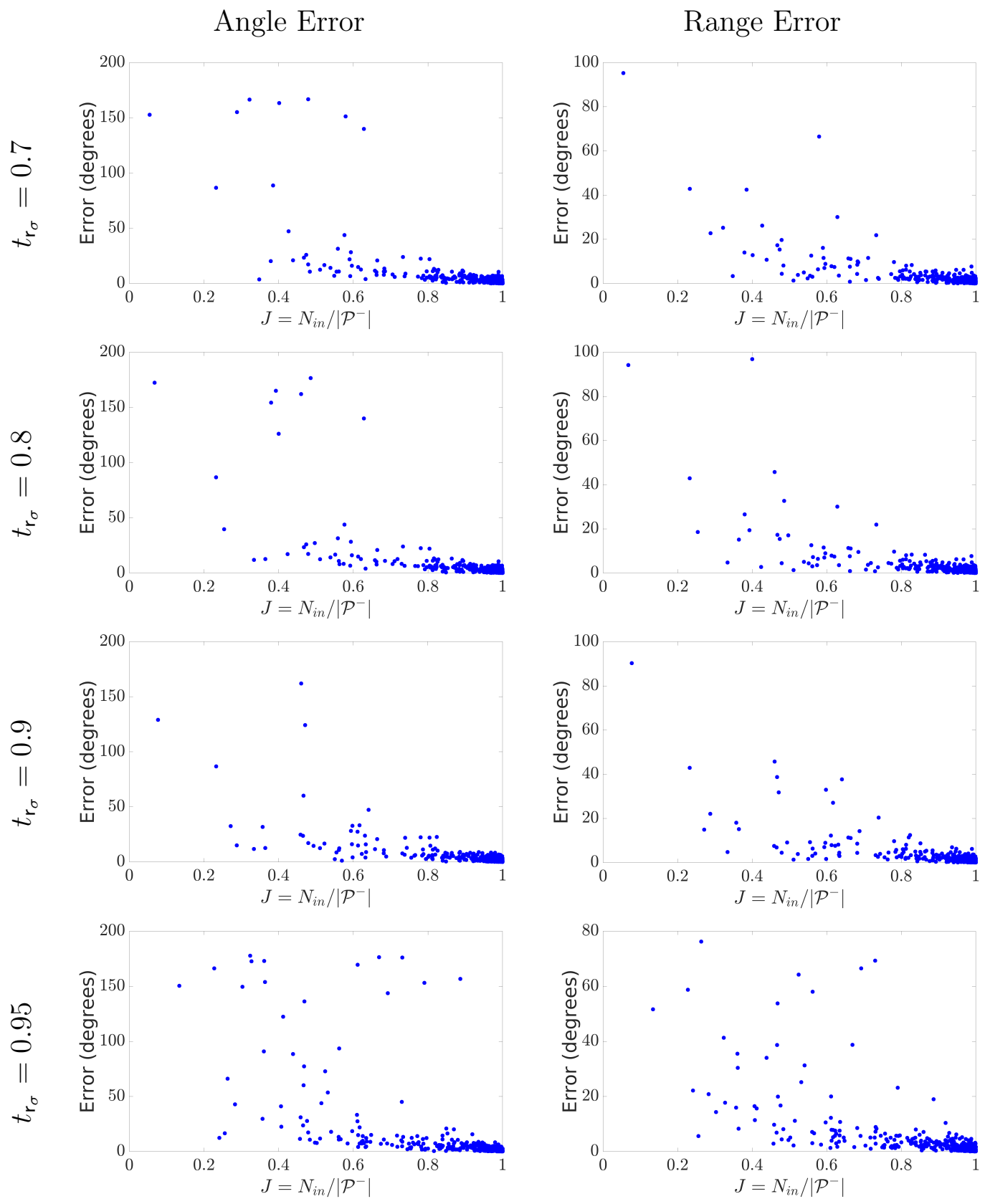

Figure 5.22: All pose estimation errors as a function of cost metric value for 500 run Monte Carlo varying scale threshold $t_{\mathrm{r}_{\sigma}}$ using distance metric JC and requiring both axes scale ratios to meet threshold. 
Single Scale Ratio
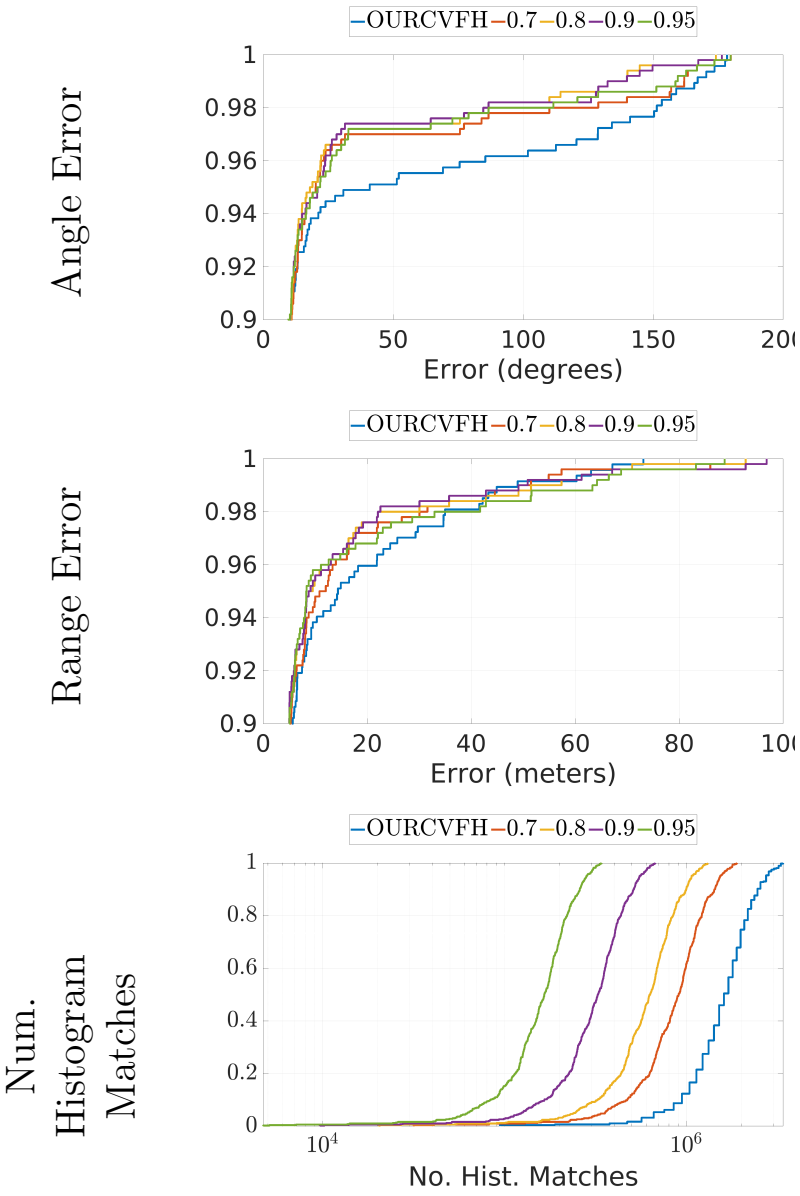

Both Scale Ratio
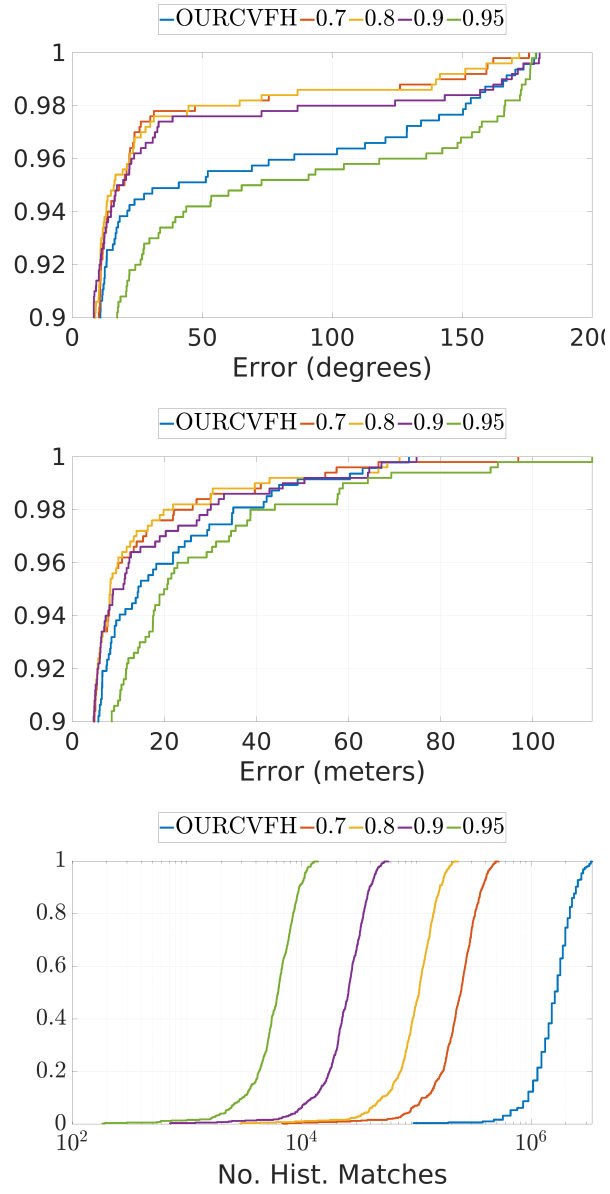

Figure 5.23: Pose estimation errors for 100 run Monte Carlo experiment for various scale thresholds using CS distance. Left column is when one axis scale ratio is greater than $t_{r_{\sigma}}$. Right column is when both scale ratios are greater than $t_{r_{\sigma}}$. Top row is the angular error in degrees, middle row is the range error in meters, bottom row is the number of histogram matches performed. 
Table 5.6: Statistics for the 500 run Monte Carlo experiment scale ratio threshold using distance metric CS.

\begin{tabular}{|c|c|c|c|c|c|}
\hline \multirow{2}{*}{\multicolumn{2}{|c|}{ OUR }} & \multicolumn{4}{|c|}{ one ratio } \\
\hline & & $t_{\mathrm{r}_{\sigma}}=0.7$ & $t_{\mathrm{r}_{\sigma}}=0.8$ & $t_{\mathrm{r}_{\sigma}}=0.9$ & $t_{\mathrm{r}_{\sigma}}=0.95$ \\
\hline \multicolumn{6}{|c|}{ Num. Pose Estimates } \\
\hline & 470 & 500 & 500 & 500 & 500 \\
\hline \multicolumn{6}{|l|}{ Angle Error } \\
\hline Mean (deg.) & $9.78^{\circ}$ & $7.41^{\circ}$ & $6.51^{\circ}$ & $6.53^{\circ}$ & $7.04^{\circ}$ \\
\hline Median (deg.) & $2.44^{\circ}$ & $1.90^{\circ}$ & $1.84^{\circ}$ & $1.81^{\circ}$ & $1.90^{\circ}$ \\
\hline Std. Dev. (deg.) & $29.12^{\circ}$ & $23.68^{\circ}$ & $19.99^{\circ}$ & $20.46^{\circ}$ & $22.39^{\circ}$ \\
\hline \multicolumn{6}{|l|}{ Range Error } \\
\hline Mean (m.) & $3.59 \mathrm{~m}$ & $3.17 \mathrm{~m}$ & $3.08 \mathrm{~m}$ & $3.05 \mathrm{~m}$ & $3.31 \mathrm{~m}$ \\
\hline Median (m.) & $1.43 \mathrm{~m}$ & $1.09 \mathrm{~m}$ & $1.08 \mathrm{~m}$ & $1.11 \mathrm{~m}$ & $1.15 \mathrm{~m}$ \\
\hline Std. Dev. (m.) & $8.52 \mathrm{~m}$ & $8.52 \mathrm{~m}$ & $8.45 \mathrm{~m}$ & $8.63 \mathrm{~m}$ & $9.33 \mathrm{~m}$ \\
\hline \multicolumn{6}{|c|}{ Num. Hist. Matches } \\
\hline Mean (m.) & $1.67 e^{6}$ & $9.12 e^{5}$ & $6.36 e^{5}$ & $3.29 e^{5}$ & $1.67 e^{9}$ \\
\hline Median (m.) & $1.60 e^{6}$ & $9.05 e^{5}$ & $6.30 e^{5}$ & $3.25 e^{5}$ & $1.65 e^{5}$ \\
\hline \multirow[t]{3}{*}{ Std. Dev. (m.) } & $6.10 e^{5}$ & $3.59 e^{5}$ & $2.51 e^{5}$ & $1.29 e^{5}$ & $6.56 e^{4}$ \\
\hline & & \multicolumn{4}{|c|}{ two ratios } \\
\hline & OUR-CVFH & $t_{\mathrm{r}_{\sigma}}=0.7$ & $t_{r_{\sigma}}=0.8$ & $t_{\mathrm{r}_{\sigma}}=0.9$ & $t_{\mathrm{r}_{\sigma}}=0.95$ \\
\hline \multicolumn{6}{|l|}{ Angle Error } \\
\hline Mean (deg.) & $9.78^{\circ}$ & $6.06^{\circ}$ & $5.96^{\circ}$ & $6.79^{\circ}$ & $11.77^{\circ}$ \\
\hline Median (deg.) & $2.44^{\circ}$ & $1.87^{\circ}$ & $1.85^{\circ}$ & $1.70^{\circ}$ & $2.08^{\circ}$ \\
\hline Std. Dev. (deg.) & $29.12^{\circ}$ & $19.21^{\circ}$ & $19.16^{\circ}$ & $23.43^{\circ}$ & $33.47^{\circ}$ \\
\hline \multicolumn{6}{|l|}{ Range Error } \\
\hline Mean (m.) & $3.59 \mathrm{~m}$ & $2.86 \mathrm{~m}$ & $2.76 \mathrm{~m}$ & $2.95 \mathrm{~m}$ & $4.51 \mathrm{~m}$ \\
\hline Median (m.) & $1.43 \mathrm{~m}$ & $1.09 \mathrm{~m}$ & $1.08 \mathrm{~m}$ & $1.04 \mathrm{~m}$ & $1.17 \mathrm{~m}$ \\
\hline Std. Dev. (m.) & $8.52 \mathrm{~m}$ & $7.60 \mathrm{~m}$ & $7.21 \mathrm{~m}$ & $7.87 \mathrm{~m}$ & $11.44 \mathrm{~m}$ \\
\hline \multicolumn{6}{|c|}{ Num. Hist. Matches } \\
\hline Mean (m.) & $1.67 e^{6}$ & $9.12 e^{5}$ & $6.36 e^{5}$ & $3.29 e^{5}$ & $1.67 e^{9}$ \\
\hline Median (m.) & $1.60 e^{6}$ & $9.05 e^{5}$ & $6.30 e^{5}$ & $3.25 e^{5}$ & $1.65 e^{5}$ \\
\hline Std. Dev. (m.) & $6.10 e^{5}$ & $3.59 e^{5}$ & $2.51 e^{5}$ & $1.29 e^{5}$ & $6.56 e^{4}$ \\
\hline
\end{tabular}



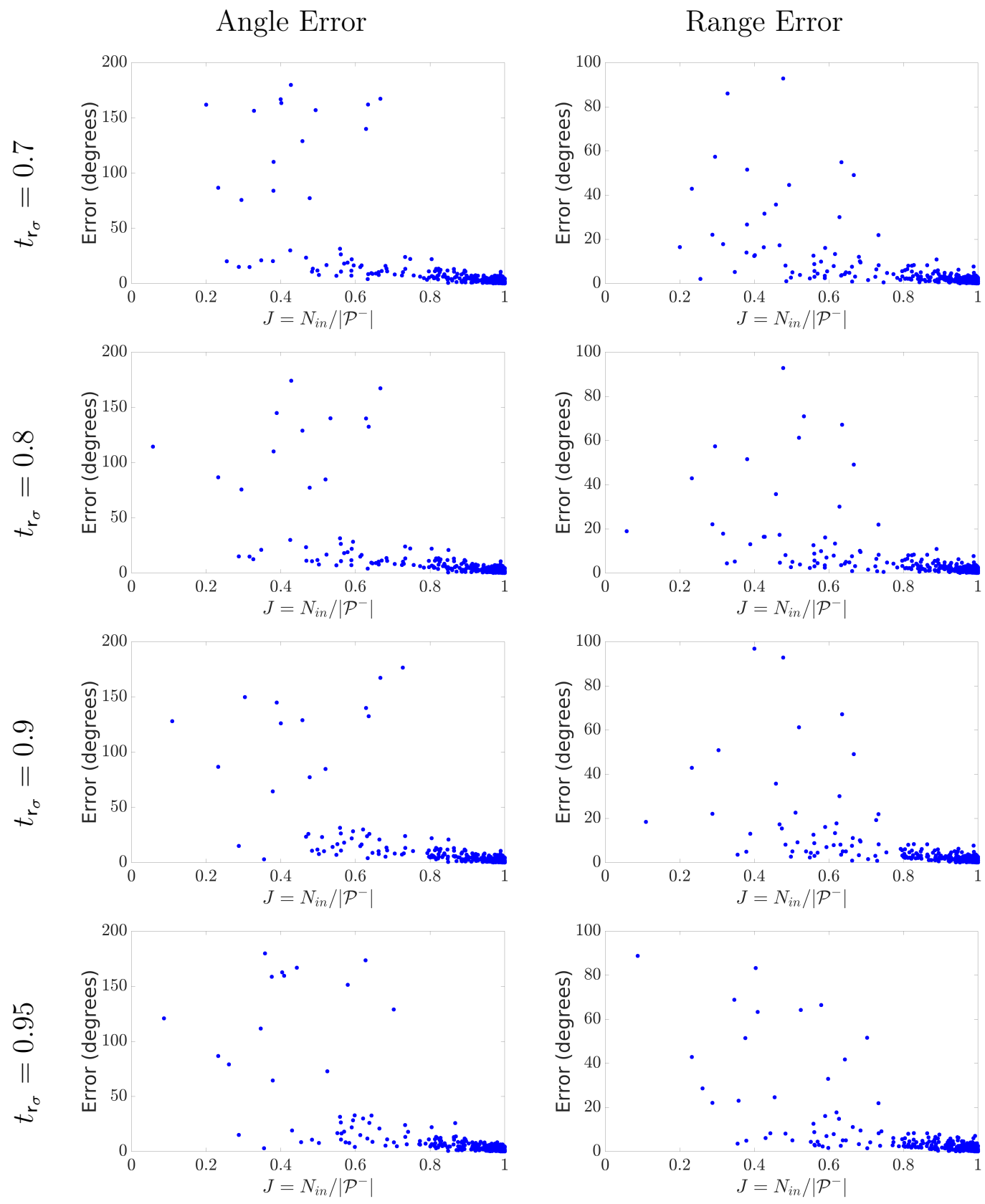

Figure 5.24: All pose estimation errors as a function of cost metric value for 500 run Monte Carlo varying scale threshold $t_{\mathrm{r}_{\sigma}}$ using distance metric CS and requiring one axis scale ratio to meet threshold.. 

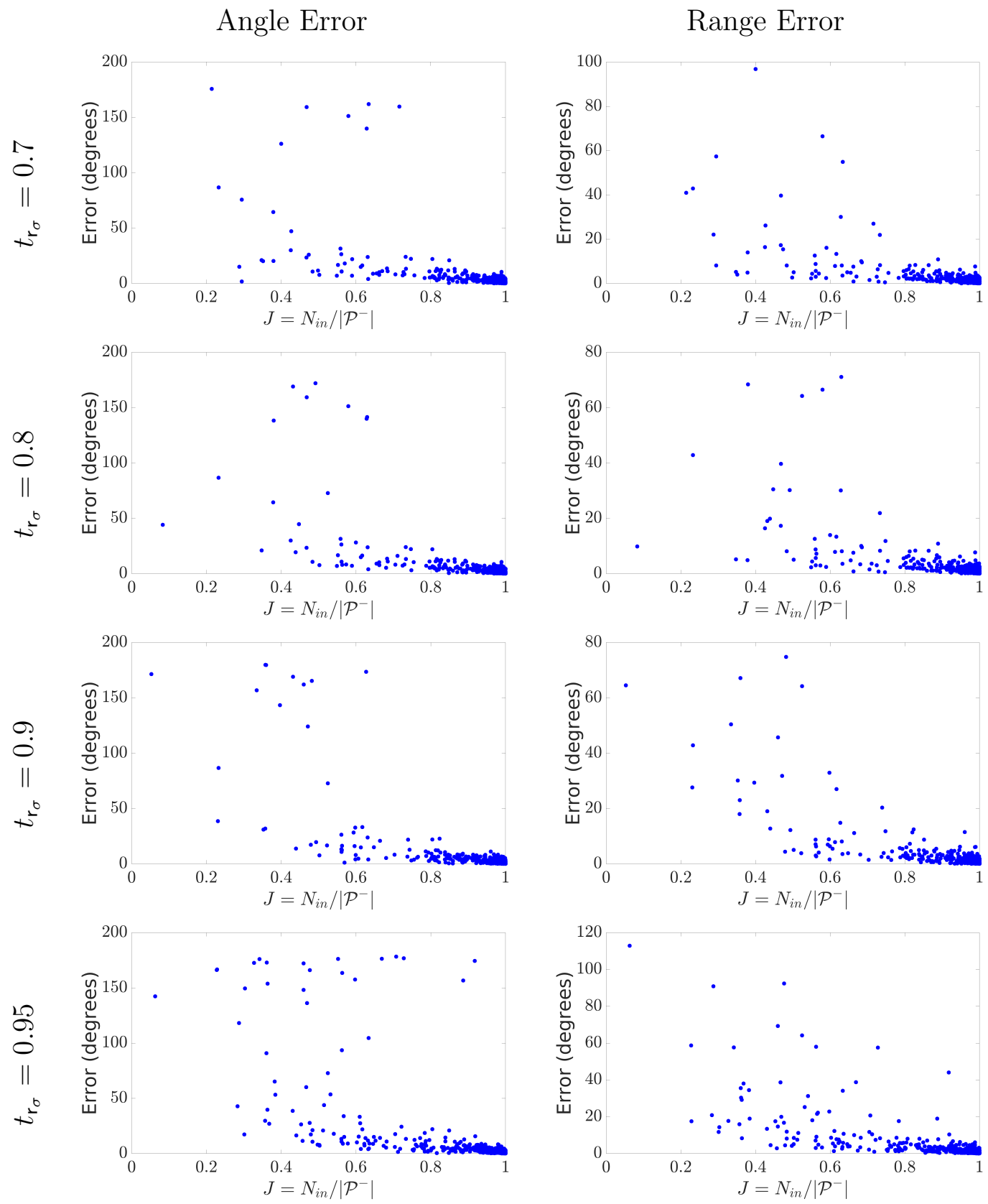

Figure 5.25: All pose estimation errors as a function of cost metric value for 500 run Monte Carlo varying scale threshold $t_{\mathrm{r}_{\sigma}}$ using distance metric CS and requiring both axes scale ratios to meet threshold. 
ing database. Computational expense is saved by performing a single value comparison of scales to eliminate improbably comparisons before matching histograms. OUR-CVFH must match every histogram, yielding higher computational expense.

All pose estimation errors as a function of cost metric value are shown for JC metric in Fig. 5.21 and Fig. 5.22, and for CS metric in Fig. 5.24 and Fig. 5.25. Notice that requiring both axes scale ratios to satisfy the threshold reduces the quantity of poor pose estimates for both JC and CS. Any poor pose estimates remaining also have a smaller cost value $J$, which will be beneficial when setting the threshold $t_{J}$. Using a scale threshold for comparing histograms makes the SOUR-CVFH descriptor more discriminatory by eliminating matches of physically different clusters. Based on these figures and the analysis, we select the Jaccard (JC) distance metric, set the scale threshold $t_{\mathrm{r}_{\sigma}}=0.9$, and require both axes scale ratios to satisfy the threshold before moving forward with histogram matching.

\subsubsection{Interpolated Spatial Octant Component (ISOC)}

The fourth component of OUR-CVFH is used to spatially describe the cluster points. This is different from the other histogram components which describe characteristics of the surface normal vector variations. Construction of the ISOC uses three 1D Gaussians placed on each of the axes with each point receiving eight weights (one weight per octant). Aldoma et al. [54] originally set the Gaussian standard deviation $\varsigma=1 \mathrm{~cm}$ with the assumption 
that the model and scene point clouds will always have the same resolution of 3 $\mathrm{mm}$. Aldoma et al. enforce this constraint by using MLS upsampling followed by downsampling to the desired resolution. We do not perform point-cloud upsampling or downsampling. Instead, we construct our database at multiple ranges to more accurately emulate the effects of sensor FOV on range images. Therefore, we must revisit the Gaussians' standard deviations for constructing the ISOC.

The Gaussian standard deviation should be dynamic so that it accounts for the object's physical size. Selecting a fixed value, like OUR-CVFH, would only be applicable to objects of that size. We discuss two options for the Gaussian standard deviation.

The first option is to set $\varsigma=\epsilon$, where $\epsilon$ is the scene point-cloud spatial resolution. With this setting, the ISOC describes the distribution of points in the cluster while being dynamic to the point-cloud resolution, which is representative of the object range as well as the sensor. This selection would impose a limitation on the use of the histogram descriptor, namely that the training database and the scene must be measured with a sensor of the same physical properties (e.g. FOV and resolution). Suppose a scene is viewed by two sensors with different resolutions, but situated with the same pose such that they return $\mathcal{P}_{1}$ and $\mathcal{P}_{2}$. Suppose the resolution of $\mathcal{P}_{1}$ returned by the first sensor is $\epsilon$ and the resolution of $\mathcal{P}_{2}$ returned by the second sensor is $2 \epsilon$. Since both sensors have the same pose and measure the same scene, the histograms should match; however, since the resulting point-cloud resolutions 


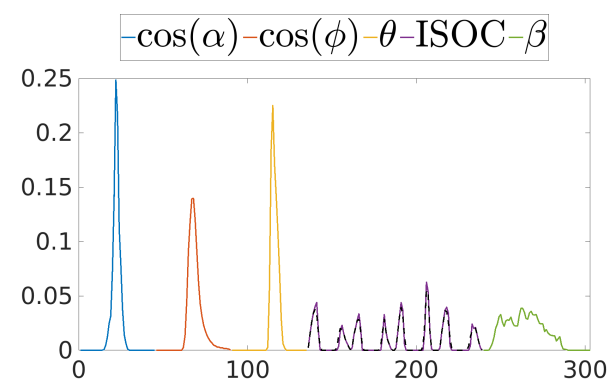

(a) Histograms of ISOC $\varsigma=0.1$ and $\operatorname{ISOC} \varsigma=\epsilon$

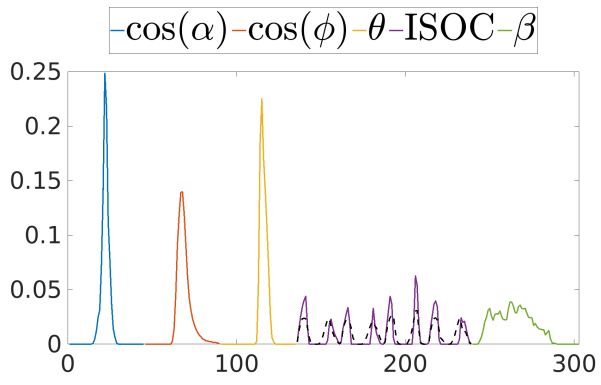

(c) Histograms of ISOC $\varsigma=0.1$ and ISOC $\varsigma_{i}=\sqrt{\lambda_{i}}$

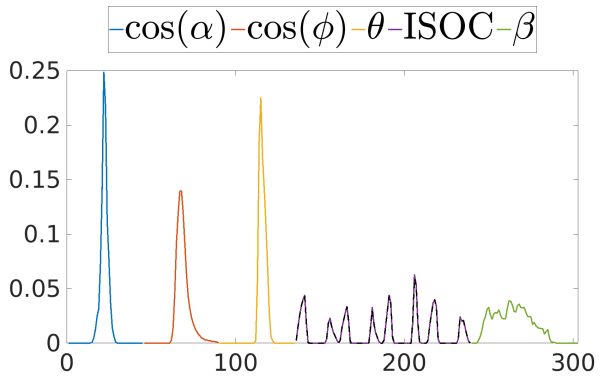

(e) Histograms of ISOC $\varsigma=0.1$ and ISOC LERP

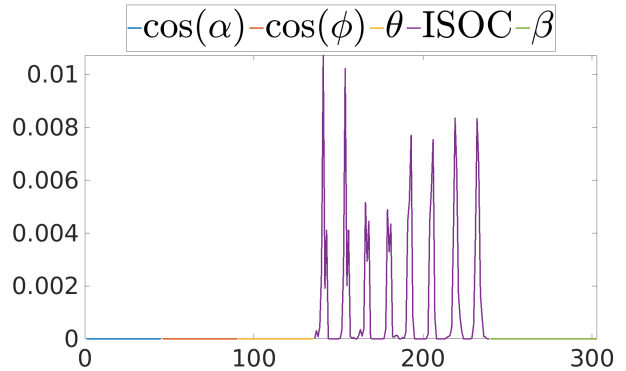

(b) Difference between histograms

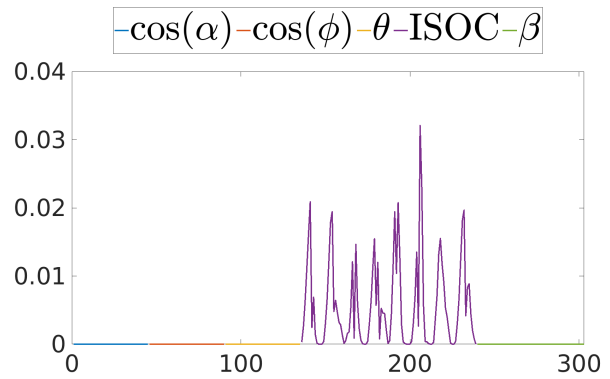

(d) Difference between histograms

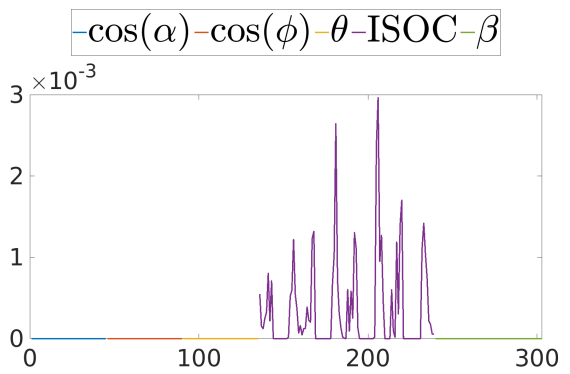

(f) Difference between histograms

Figure 5.26: Normalized histograms with various settings of ISOC weighting. Left column is the histogram with all modifications up until this step and overlaid by a histogram with the various ISOC settings. Right column is the absolute difference between the two histograms. Notice the only difference is with ISOC. 
are different, the Gaussian standard deviations are different, and their ISOC histograms will not match. This results in a failed pose estimate for this scene. Thus, the training sensor must carry the same physical properties (e.g. FOV and resolution) as the active sensor for the histograms to accurately match. OUR-CVFH with our multi-distance training routine is also constrained by this requirement because it has an unnormalized histogram. As the LIDAR resolution increases, so does the histogram height since it depends on the number of points in the cluster. The same sensor must be used in training and active sensing for OUR-CVFH as well. Therefore, we have not introduced a new restriction, only substituted the reasoning.

The second option is to set $\varsigma_{i}=\sqrt{\lambda_{i}}$, where $\lambda_{i}$ are the eigenvalues of a clusters' scatter matrix decomposition. This setting is also dynamic, but consistent across a given clusters regardless of sensor resolution. Cluster scale is an inherent quality, fixed for all other inputs, and is a scaled version of $\lambda_{i}$ (see Sec. 5.2.6). The ISOC's purpose is to spatially describe a cluster's points location uncertainty in each axes octant. A cluster may have a different scale along each axes, implying that the points' location uncertainty is different for each axes and that the Gaussian weights should be unique to each axes. Therefore, we place a Gaussian on each axes with a standard deviation equal to the cluster's axis eigenvalue square root, $\varsigma_{i}=\sqrt{\lambda_{i}}$. These Gaussians may be different for each axes. With this weighting, we are actually describing the spatial distribution of points across the octants without reference to the resolution. Recall the previous scenario of two sensors with different physical 


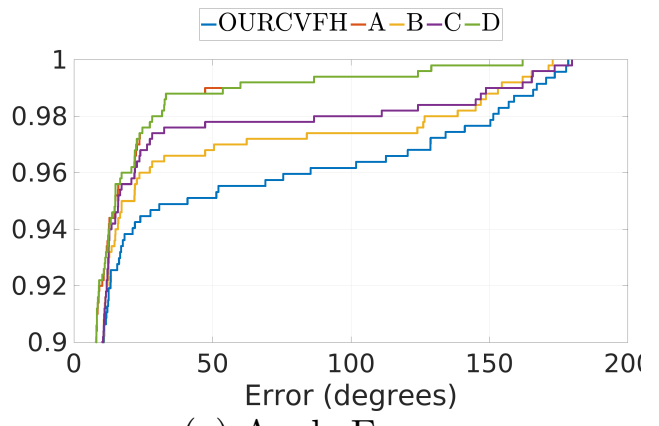

(a) Angle Error

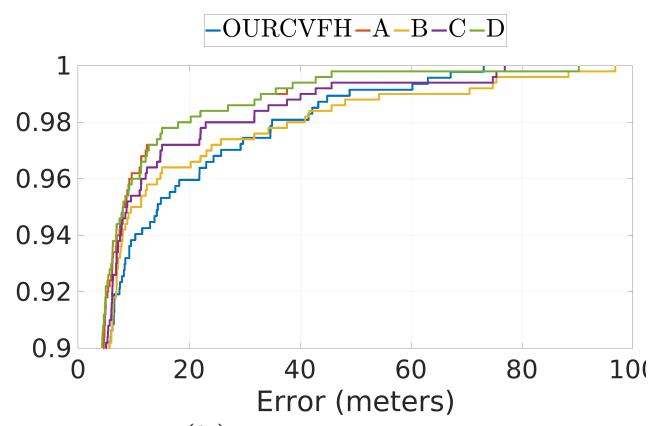

(b) Range Error

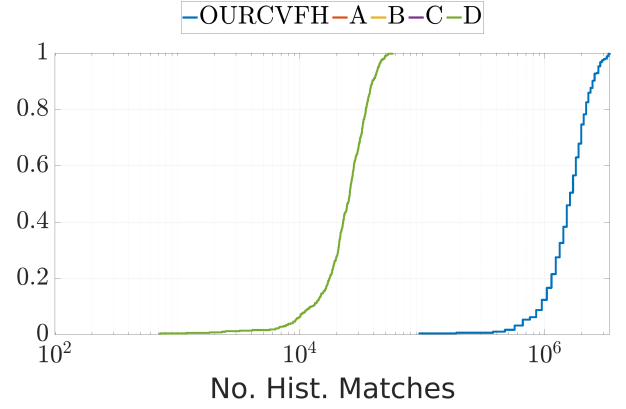

(c) Num. Histogram Matches

Figure 5.27: Pose estimation errors without outliers for 500 run Monte Carlo experiment various ISOC weighting using metric JC. Methods A, B, and C use all the modifications up to this point. Method A uses $\varsigma=0.01$, method B uses $\varsigma=\epsilon$, method $\mathrm{C}$ uses $\varsigma_{i}=\sqrt{\lambda_{i}}$, and method D uses LERP. 
Table 5.7: Statistics for the 500 run Monte Carlo experiment varying ISOC weighting using metric JC. Method A uses $\varsigma=0.01$, method B uses $\varsigma=\epsilon$, method C uses $\varsigma_{i}=\sqrt{\lambda_{i}}$, and method D uses LERP.

\begin{tabular}{lrrrrr}
\hline & OUR-CVFH & $\mathrm{A}$ & $\mathrm{B}$ & $\mathrm{C}$ & $\mathrm{D}$ \\
\hline Num. Pose Estimates & & & & & \\
& 470 & 500 & 500 & 500 & 500 \\
\hline Angle Error & & & & & \\
Mean (deg.) & $9.78^{\circ}$ & $4.46^{\circ}$ & $7.52^{\circ}$ & $6.64^{\circ}$ & $4.38^{\circ}$ \\
Median (deg.) & $2.44^{\circ}$ & $1.63^{\circ}$ & $1.67^{\circ}$ & $1.82^{\circ}$ & $1.58^{\circ}$ \\
Std. Dev. (deg.) & $29.12^{\circ}$ & $12.61^{\circ}$ & $24.24^{\circ}$ & $21.91^{\circ}$ & $12.65^{\circ}$ \\
\hline Range Error & & & & & \\
Mean (m.) & $3.59 \mathrm{~m}$ & $2.49 \mathrm{~m}$ & $3.44 \mathrm{~m}$ & $2.95 \mathrm{~m}$ & $2.43 \mathrm{~m}$ \\
Median (m.) & $1.43 \mathrm{~m}$ & $1.00 \mathrm{~m}$ & $1.12 \mathrm{~m}$ & $1.16 \mathrm{~m}$ & $0.97 \mathrm{~m}$ \\
Std. Dev. (m.) & $8.52 \mathrm{~m}$ & $6.30 \mathrm{~m}$ & $9.86 \mathrm{~m}$ & $7.65 \mathrm{~m}$ & $6.28 \mathrm{~m}$ \\
\hline Num. Hist. Matches & & & & & \\
Mean (m.) & $1.67 e^{6}$ & $2.58 e^{4}$ & $2.58 e^{4}$ & $2.58 e^{4}$ & $2.58 e^{4}$ \\
Median (m.) & $1.60 e^{6}$ & $2.55 e^{4}$ & $2.55 e^{4}$ & $2.55 e^{4}$ & $2.55 e^{4}$ \\
Std. Dev. (m.) & $6.10 e^{5}$ & $1.03 e^{4}$ & $1.03 e^{4}$ & $1.03 e^{4}$ & $1.03 e^{4}$ \\
\hline \hline
\end{tabular}



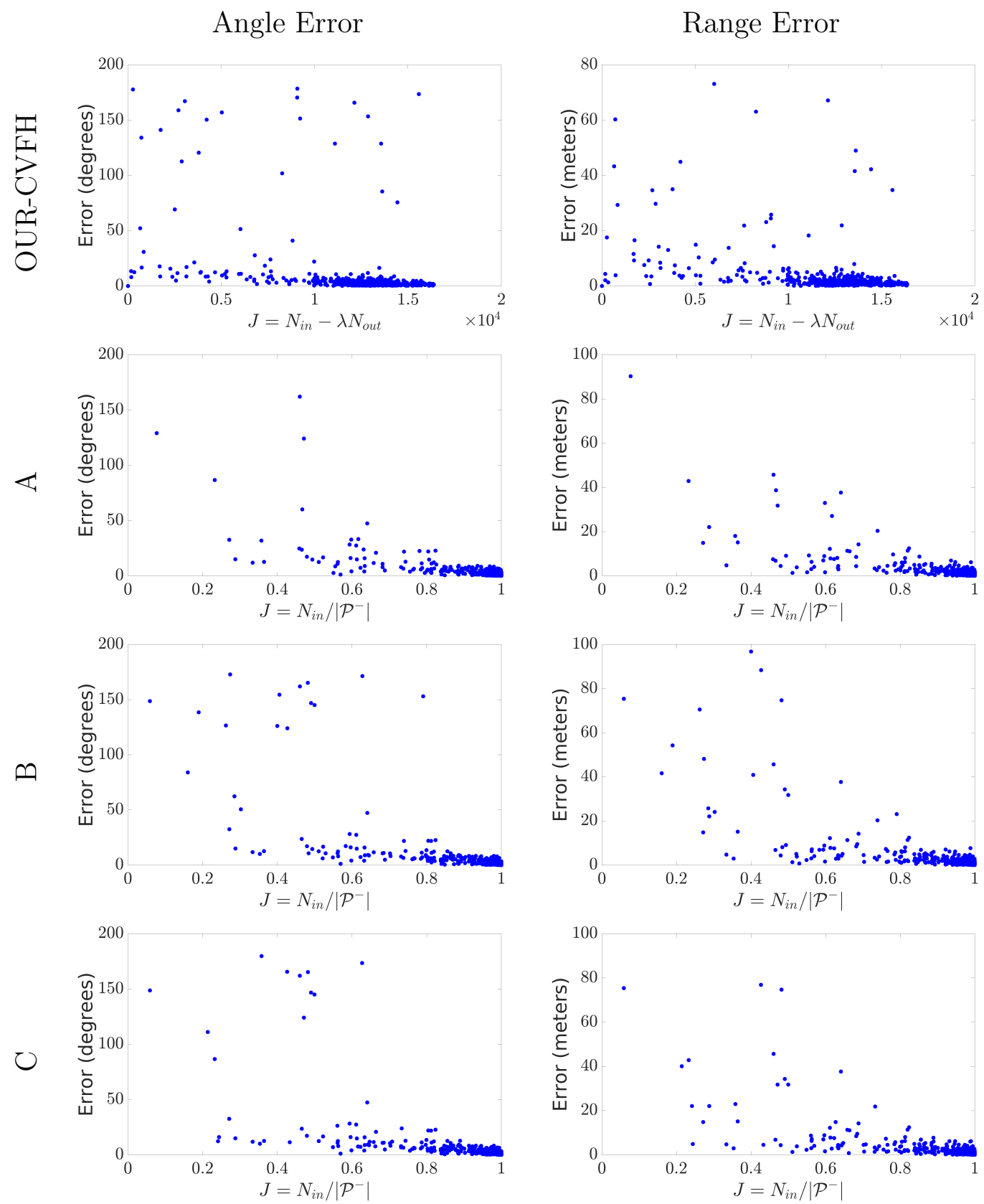

Figure 5.28: All pose estimation errors as a function of cost metric value for 500 run Monte Carlo varying ISOC weighting using metric JC. Methods A, B, $\mathrm{C}$, and D use all the modifications up to this point. Method A uses $\varsigma=0.01$, method B uses $\varsigma=\epsilon$, method $\mathrm{C}$ uses $\varsigma_{i}=\sqrt{\lambda_{i}}$, and method D uses LERP. 

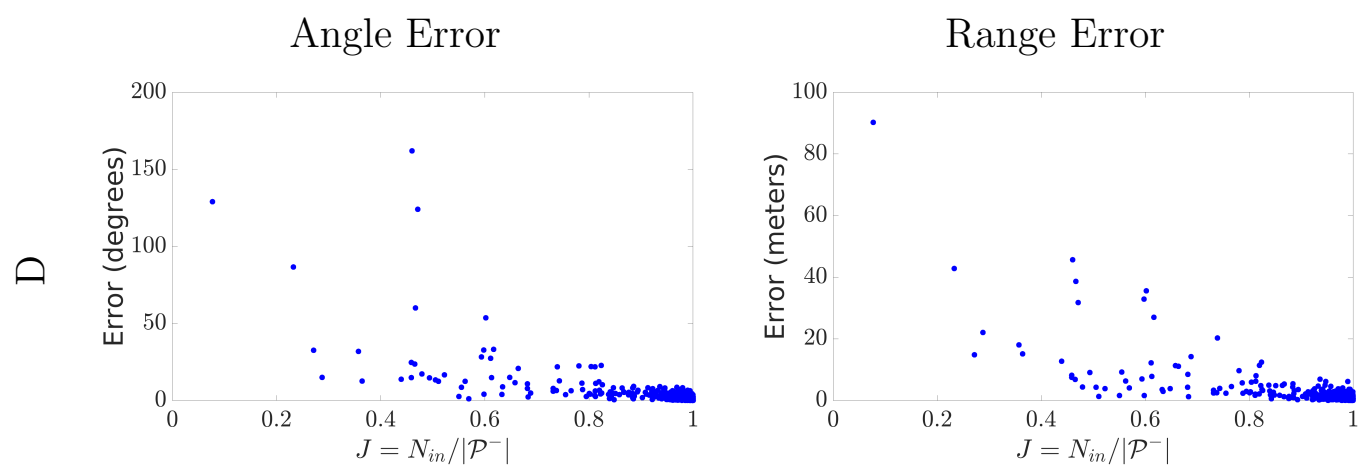

Figure 5.28: Continued

properties measuring the same scene resulting in the point-clouds $\mathcal{P}_{1}$ and $\mathcal{P}_{2}$. Both point-clouds contain the cluster $\mathcal{C}$, but with a different number of points. Since the cluster scale is fixed, and the histogram is normalized, the ISOC (and the other histogram components) will match.

Upon analysis of the results, we found that the OUR-CVFH selection of $\varsigma=0.01 \mathrm{~cm}$ performed the best. This is counter-intuitive because it is the least descriptive as the resolution of our measured point-clouds were often greater than $4 \varsigma$ yielding Gaussian weight values of nearly 0. The ISOC counted the quantity of points that fell withing a specific bin within an octant. The Gaussian weights yielded minimal affect on ISOC construction. It would be simpler and less computationally expensive to simply count the number of points in a bin for each octant for ISOC construction. However, it is desired to maintain some interpolation across bins to deal with spurious affects (e.g. measurement noise or clutter). Therefore, the third option is to use LERP (see Sec. 5.2.1) for ISOC construction. 
Fig. 5.26 shows the histograms with the various settings of ISOC weighting scheme. The left column shows the histogram with all modifications up to this point with ISOC $\varsigma=0.1$. They are overlaid by histograms with the three new settings for ISOC. The right column is the absolute difference between the overlaid histograms. Notice that ISOC LERP is nearly identical to that of ISOC $\varsigma=0.1$, but is quicker to compute.

Pose estimation errors as a CDF are shown in Fig. 5.27. We compare the affects of the three Gaussian standard deviations settings and LERP on ISOC construction to OUR-CVFH . We include all previous modifications and settings up to this point for methods A using $\varsigma=0.01 \mathrm{~cm}$, method B using $\varsigma=\epsilon$, method $\mathrm{C}$ using $\varsigma_{i}=\sqrt{\lambda_{i}}$, and method D using LERP. Notice that OURCVFH is the worst performing of the other methods. Method A performs the best and is nearly identical to method D, showing the equivalence of those approaches. Method $\mathrm{C}$ using $\varsigma_{i}=\sqrt{\lambda_{i}}$ is the next best method.

All pose estimates as a function of cost metric value are shown in Fig. 5.28. Method B produces the most outlier estimates, while methods A and D appear to produce the most accurate measurements. Statistics regarding the performance of these methods are listed in Tab. 5.7. OUR-CVFH performs the worst, while method D yields the best statistics. Recall that we are not yet applying the cost metric threshold $t_{J}$, for which it would improve the results of methods A, B, C, and D, but reduce the number of pose estimates returned.

Also considered is the effect of sensing objects with a different physical system than that used in training. Specifically, we use a simulated LIDAR 
Table 5.8: Statistics for the 500 run Monte Carlo experiment varying ISOC weighting and varying resolution using metric JC. Method A uses $\varsigma=0.01$, method B uses $\varsigma=\epsilon$, method $\mathrm{C}$ uses $\varsigma_{i}=\sqrt{\lambda_{i}}$, and method D uses LERP.

\begin{tabular}{lrrrrrr}
\hline \multicolumn{2}{c}{ OUR-CVFH } & A & B & C & D \\
\hline \multicolumn{1}{c}{ Num. Pose Estimates } & & & & & \\
& 256 & 447 & 432 & 430 & 446 \\
\hline Angle Error & & & & & \\
Mean (deg.) & $12.44^{\circ}$ & $4.20^{\circ}$ & $4.07^{\circ}$ & $3.60^{\circ}$ & $3.12^{\circ}$ \\
Median (deg.) & $0.98^{\circ}$ & $0.71^{\circ}$ & $0.76^{\circ}$ & $0.78^{\circ}$ & $0.72^{\circ}$ \\
Std. Dev. (deg.) & $37.45^{\circ}$ & $19.48^{\circ}$ & $18.65^{\circ}$ & $16.13^{\circ}$ & $14.51^{\circ}$ \\
\hline Range Error & & & & & \\
Mean (m.) & $4.15 \mathrm{~m}$ & $2.06 \mathrm{~m}$ & $2.06 \mathrm{~m}$ & $1.86 \mathrm{~m}$ & $1.63 \mathrm{~m}$ \\
Median (m.) & $0.66 \mathrm{~m}$ & $0.51 \mathrm{~m}$ & $0.50 \mathrm{~m}$ & $0.52 \mathrm{~m}$ & $0.50 \mathrm{~m}$ \\
Std. Dev. (m.) & $11.39 \mathrm{~m}$ & $8.30 \mathrm{~m}$ & $8.19 \mathrm{~m}$ & $6.20 \mathrm{~m}$ & $6.50 \mathrm{~m}$ \\
\hline \hline
\end{tabular}

with a resolution of $256 \times 256$ while maintaining a FOV of $20^{\circ}$. We still use the same training dataset as explained at the beginning of Sec. 5.2. For this example, we also apply a cost metric threshold of $t_{J}=0.4$. Fig. 5.29 shows a CDF of the pose estimation errors with the applied cost metric threshold. Statistics for the performance of these method are listed in Tab 5.8. OURCVFH returns the fewest number of pose estimates and the worst estimates. Performance of methods A, B, C, and D are similar. All pose estimates as function of cost metric are shown in Fig. 5.30 with a vertical dashed line to denote the cost metric threshold $t_{J}$.

Although method C using $\varsigma_{i}=\sqrt{\lambda_{i}}$ provides a consistent representation 


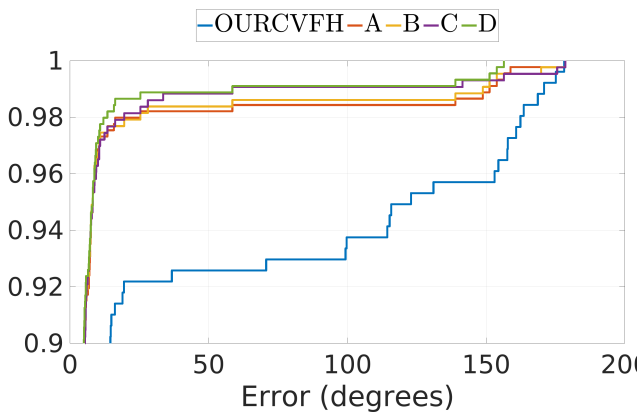

(a) Angle Error

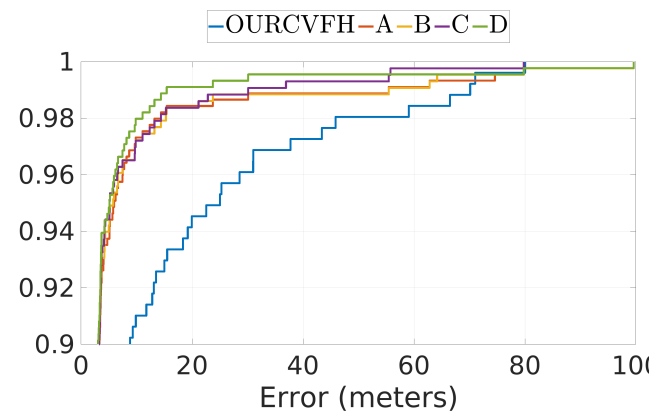

(b) Range Error

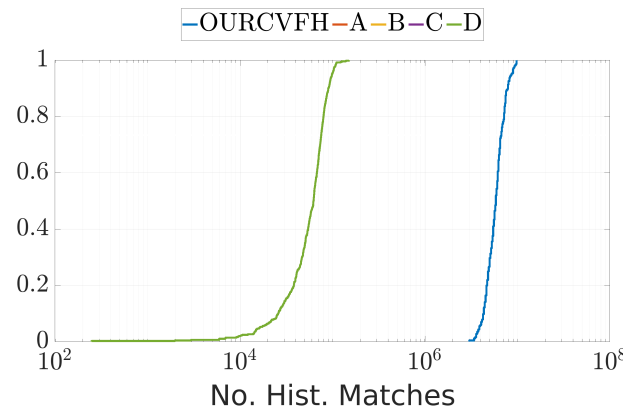

(c) Num. Histogram Matches

Figure 5.29: Pose estimation errors without outliers for 500 run Monte Carlo experiment various ISOC weighting and varying resolution using metric JC. Methods A, B, and C use all the modifications up to this point. Method A uses $\varsigma=0.01$, method B uses $\varsigma=\epsilon$, method $\mathrm{C}$ uses $\varsigma_{i}=\sqrt{\lambda_{i}}$, and method D uses LERP. 

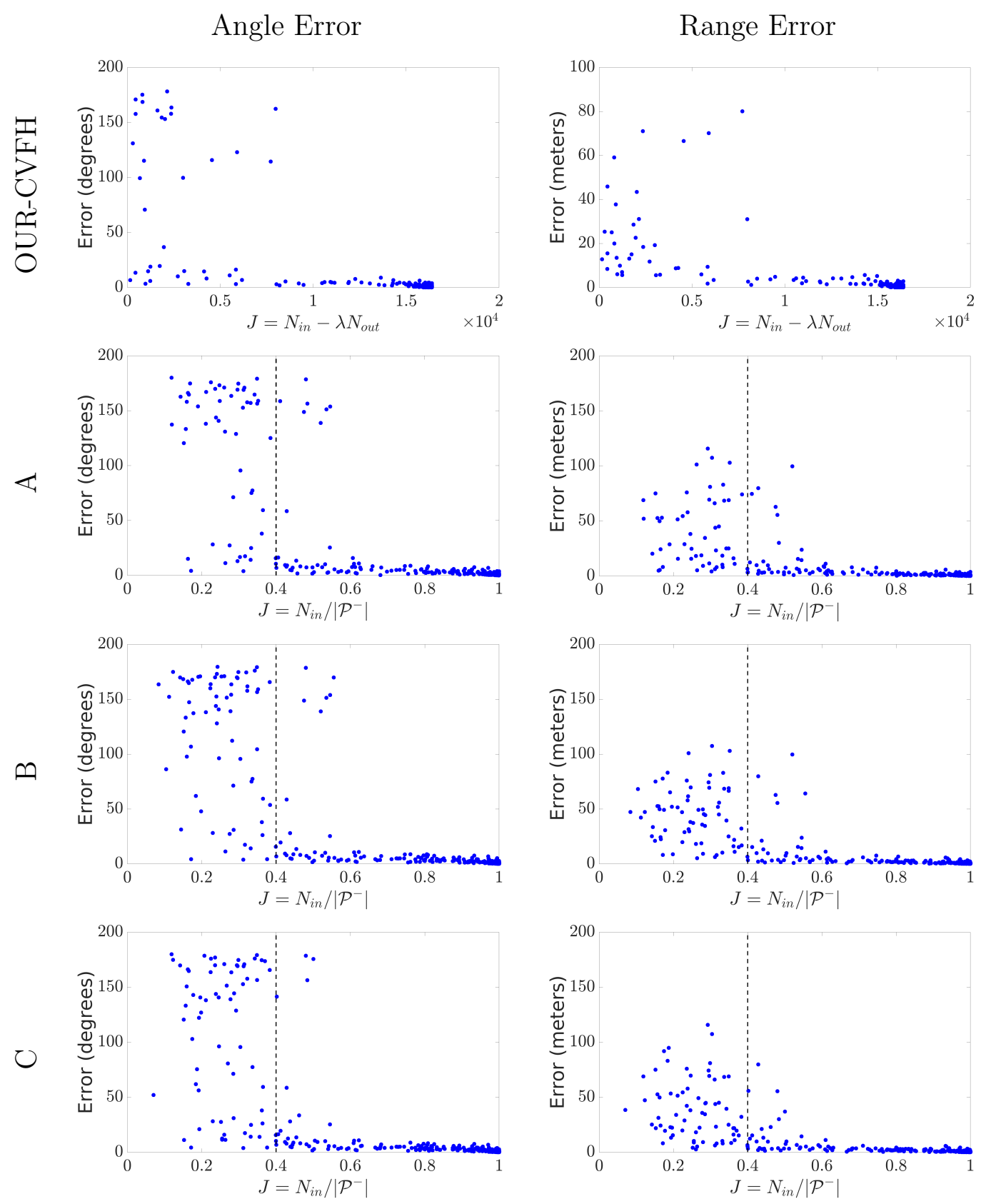

Figure 5.30: All pose estimation errors as a function of cost metric value for 500 run Monte Carlo varying ISOC weighting and varying resolution using metric JC. Methods A, B, and $\mathrm{C}$ use all the modifications up to this point. Method A uses $\varsigma=0.01$, method B uses $\varsigma=\epsilon$, method $\mathrm{C}$ uses $\varsigma_{i}=\sqrt{\lambda_{i}}$, and method D uses LERP. Vertical dashed line signifies the cost metric threshold of $t_{J}=0.4$. 

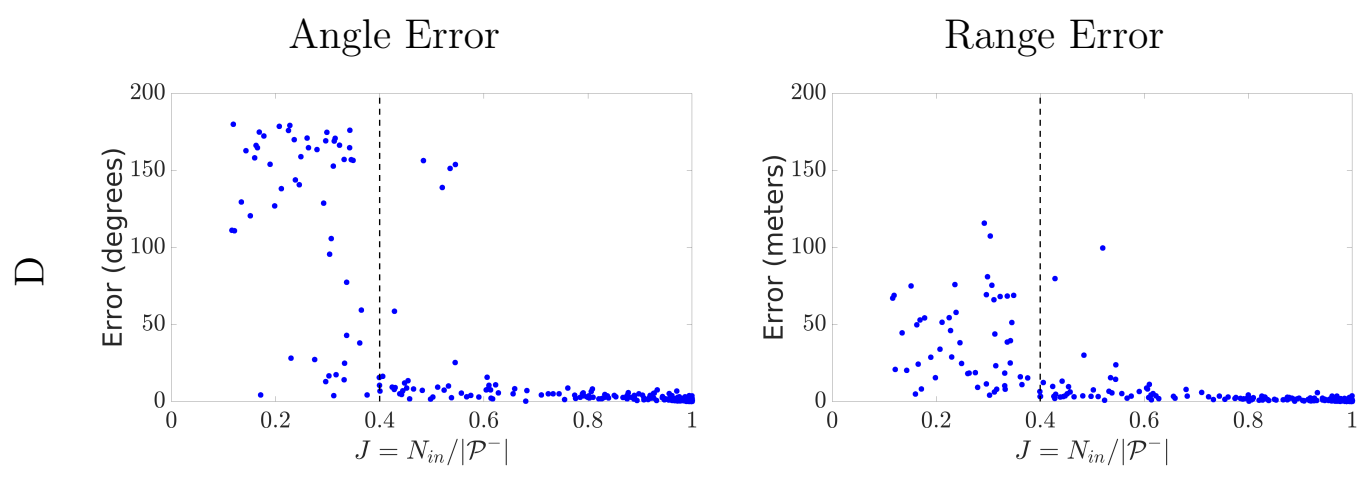

Figure 5.30: Continued

of the clusters that does not vary with sensor resolution or object distance, it is out-performed by the much simpler LERP. A close look at Fig. 5.29 shows that methods A, B, C, and D perform similarly.

\subsubsection{Uniqueness of Histogram Components}

The OUR-CVFH descriptor is constructed from five individual histograms appended together: $\mathcal{D}=[\alpha, \phi, \theta$, ISOC, $\beta]$. Four of the components describe angular distributions, while ISOC describes the physical distribution of points on the cluster. While OUR-CVFH and our SOUR-CVFH use all five of the components, they may offer different accuracies if used independently. This section serves to characterize the performance of the histogram components individually and in combinations other than that provided in OURCVFH . Our goal is to determine if the SOUR-CVFH descriptor may be shortened by component, or if all five components are necessary.

We tested the pose estimation accuracy for various combinations of components as shown in Table 5.9 where the blue marks if the component is 
included in the method. All other specifications of SOUR-CVFH are used: (1) linear interpolation of each feature, (2) describing $\beta$ in angular space, (3) using cost metric of Eq. 5.13, (4) normalize each histogram component to unity, (5) the distance metric JC, (6) require both scale ratios to satisfy the threshold $t_{\mathrm{r}}=0.9,(5)$ and test ISOC with LERP and and Gaussian standard deviations of $\varsigma_{i}=\lambda_{i}$. A CDF plot of select methods is shown in Fig. 5.31 with all the statistics shown Table 5.9.

The best performing method is $\mathrm{G}$ in green which only uses scale, $\cos (\alpha)$, and ISOC LERP. The second best performing is method A shown in red, which is SOUR-CVFH . Methods D, I, and U perform comparable to OUR-CVFH . Notice that using any of $\cos (\alpha), \cos (\phi), \theta$, or $\beta$ individually, or with scale, (e.g. Methods M, N, O, P, Q, R, S, and T) are incapable of accurately measuring pose. All methods that include ISOC with LERP offers decent pose estimation capabilities. This analysis shows that the most important histogram component is ISOC. Performance of particular combinations improves upon the ISOC only method.

All pose estimation errors as a function of cost metric for the select methods A, C, D, G, I, and U are shown in Fig. 5.32. Mtehods D, I, and U perform most similarly to OUR-CVFH . Method G produces the fewest number of outlier measurements closely followed by Method A (SOUR-CVFH ). This outliers would be efficiently eliminated by enforcing the cost metric threshold of $t_{J}=0.4$ or with navigation filter rejection. 


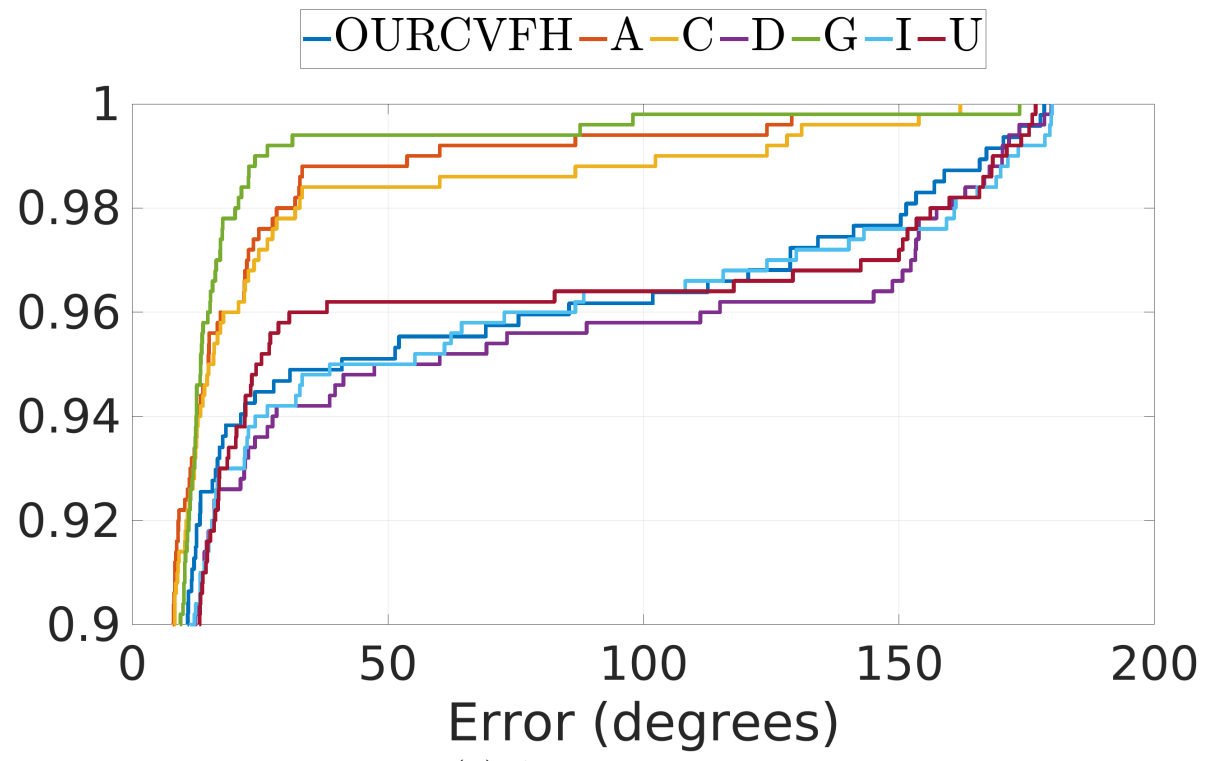

(a) Angle Error

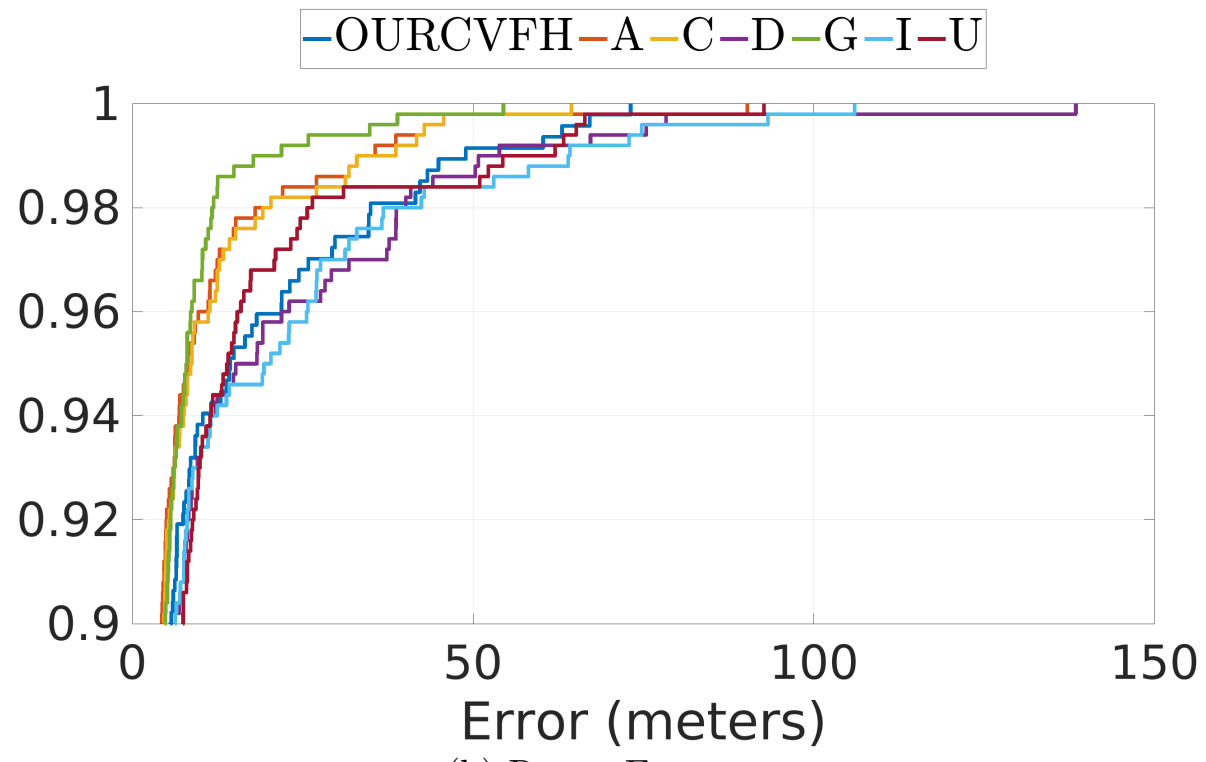

(b) Range Error

Figure 5.31: Pose estimation errors for 500 run Monte Carlo experiment for various histogram component combinations using JC distance for select method A, C, D, G, I, and U. 
Table 5.9: Various histogram component combinations tested with 500 run Monte Carlo using distance metric JC.

\begin{tabular}{|c|c|c|c|c|c|c|c|c|c|}
\hline & OUR-CVFH & $\mathrm{A}$ & $\mathrm{B}$ & $\mathrm{C}$ & $\mathrm{D}$ & $\mathrm{E}$ & $\mathrm{F}$ & $\mathrm{G}$ & $\mathrm{H}$ \\
\hline \multicolumn{10}{|l|}{ Components } \\
\hline \multicolumn{10}{|l|}{$\begin{array}{r}\sigma_{i} \\
\cos (\alpha)\end{array}$} \\
\hline \multicolumn{10}{|l|}{$\cos (\phi)$} \\
\hline \\
\hline \multicolumn{10}{|l|}{ ISOC LERP } \\
\hline \multicolumn{10}{|l|}{$\mathrm{ISOC} \varsigma_{i}=\lambda_{i}$} \\
\hline \\
\hline \multicolumn{10}{|l|}{ Angle Error } \\
\hline Mean (deg.) & $9.78^{\circ}$ & $4.38^{\circ}$ & $6.64^{\circ}$ & $5.18^{\circ}$ & $10.63^{\circ}$ & $6.73^{\circ}$ & $15.31^{\circ}$ & $4.03^{\circ}$ & $5.79^{\circ}$ \\
\hline Median (deg.) & $2.44^{\circ}$ & $1.58^{\circ}$ & $1.82^{\circ}$ & $1.67^{\circ}$ & $1.92^{\circ}$ & $1.82^{\circ}$ & $2.28^{\circ}$ & $1.85^{\circ}$ & $2.07^{\circ}$ \\
\hline Std. Dev. (deg.) & $29.12^{\circ}$ & $12.65^{\circ}$ & $21.91^{\circ}$ & $15.76^{\circ}$ & $31.95^{\circ}$ & $21.56^{\circ}$ & $39.08^{\circ}$ & $10.43^{\circ}$ & $18.58^{\circ}$ \\
\hline \multicolumn{10}{|l|}{ Range Error } \\
\hline Mean (m.) & $3.59 \mathrm{~m}$ & $2.43 \mathrm{~m}$ & $2.95 \mathrm{~m}$ & $2.55 \mathrm{~m}$ & $3.98 \mathrm{~m}$ & $3.24 \mathrm{~m}$ & $5.69 \mathrm{~m}$ & $2.17 \mathrm{~m}$ & $2.52 \mathrm{~m}$ \\
\hline Median (m.) & $1.43 \mathrm{~m}$ & $0.97 \mathrm{~m}$ & $1.17 \mathrm{~m}$ & $1.05 \mathrm{~m}$ & $1.21 \mathrm{~m}$ & $1.10 \mathrm{~m}$ & $1.28 \mathrm{~m}$ & $0.99 \mathrm{~m}$ & $1.15 \mathrm{~m}$ \\
\hline Std. Dev. (m.) & $8.52 \mathrm{~m}$ & $6.28 \mathrm{~m}$ & $7.65 \mathrm{~m}$ & $5.82 \mathrm{~m}$ & $10.82 \mathrm{~m}$ & $8.64 \mathrm{~m}$ & $13.43 \mathrm{~m}$ & $4.25 \mathrm{~m}$ & $4.73 \mathrm{~m}$ \\
\hline
\end{tabular}


Table 5.9: Continued

\begin{tabular}{|c|c|c|c|c|c|c|c|c|c|}
\hline & $\mathrm{I}$ & $\mathrm{J}$ & $\mathrm{K}$ & $\mathrm{L}$ & M & $\mathrm{N}$ & $\mathrm{O}$ & $\mathrm{P}$ & $\mathrm{Q}$ \\
\hline \multicolumn{10}{|l|}{ Components } \\
\hline \\
\hline \multicolumn{10}{|l|}{$\cos (\alpha)$} \\
\hline \multicolumn{10}{|l|}{$\cos (\phi)$} \\
\hline \multicolumn{10}{|l|}{$\theta$} \\
\hline \multicolumn{10}{|l|}{ ISOC LERP } \\
\hline \multicolumn{10}{|l|}{ ISOC $\varsigma_{i}=\lambda_{i}$} \\
\hline & & & & & & & & & \\
\hline \multicolumn{10}{|l|}{ Angle Error } \\
\hline Mean (deg.) & $9.85^{\circ}$ & $20.00^{\circ}$ & $5.56^{\circ}$ & $9.4^{\circ}$ & $53.5^{\circ}$ & $32.9^{\circ}$ & $66.6^{\circ}$ & $56.1^{\circ}$ & $115.5^{\circ}$ \\
\hline Median (deg.) & $2.01^{\circ}$ & $2.71^{\circ}$ & $1.99^{\circ}$ & $2.29^{\circ}$ & $10.18^{\circ}$ & $4.66^{\circ}$ & $22.13^{\circ}$ & $10.89^{\circ}$ & $142.83^{\circ}$ \\
\hline Std. Dev. (deg.) & $30.07^{\circ}$ & $44.55^{\circ}$ & $17.86^{\circ}$ & $28.63^{\circ}$ & $67.32^{\circ}$ & $57.54^{\circ}$ & $71.70^{\circ}$ & $68.81^{\circ}$ & $59.18^{\circ}$ \\
\hline \multicolumn{10}{|l|}{ Range Error } \\
\hline Mean (m.) & $3.94 \mathrm{~m}$ & $6.80 \mathrm{~m}$ & $2.58 \mathrm{~m}$ & $3.42 \mathrm{~m}$ & $17.01 \mathrm{~m}$ & $9.86 \mathrm{~m}$ & $18.42 \mathrm{~m}$ & $17.53 \mathrm{~m}$ & $35.35 \mathrm{~m}$ \\
\hline Median (m.) & $1.19 \mathrm{~m}$ & $1.54 \mathrm{~m}$ & $1.06 \mathrm{~m}$ & $1.30 \mathrm{~m}$ & $5.87 \mathrm{~m}$ & $3.02 \mathrm{~m}$ & $10.29 \mathrm{~m}$ & $7.04 \mathrm{~m}$ & $30.61 \mathrm{~m}$ \\
\hline Std. Dev. (m.) & $10.73 \mathrm{~m}$ & $14.66 \mathrm{~m}$ & $5.74 \mathrm{~m}$ & $6.94 \mathrm{~m}$ & $22.09 \mathrm{~m}$ & $16.87 \mathrm{~m}$ & $21.65 \mathrm{~m}$ & $21.56 \mathrm{~m}$ & $24.61 \mathrm{~m}$ \\
\hline
\end{tabular}


Table 5.9: Continued

\begin{tabular}{|c|c|c|c|c|c|}
\hline & $\mathrm{R}$ & $\mathrm{S}$ & $\mathrm{T}$ & $\mathrm{U}$ & $\mathrm{V}$ \\
\hline \multicolumn{6}{|l|}{ Components } \\
\hline \\
\hline \multicolumn{6}{|l|}{$\cos (\alpha)$} \\
\hline \multicolumn{6}{|l|}{$\cos (\phi)$} \\
\hline \\
\hline \multicolumn{6}{|l|}{ ISOC LERP } \\
\hline \multicolumn{6}{|l|}{$\mathrm{ISOC} \varsigma_{i}=\lambda_{i}$} \\
\hline \multicolumn{6}{|l|}{$\beta$} \\
\hline \multicolumn{6}{|l|}{ Angle Error } \\
\hline Mean (deg.) & $103.8^{\circ}$ & $111.8^{\circ}$ & $112.9^{\circ}$ & $9.6^{\circ}$ & $19.1^{\circ}$ \\
\hline Median (deg.) & $130.91^{\circ}$ & $141.22^{\circ}$ & $141.57^{\circ}$ & $2.21^{\circ}$ & $2.93^{\circ}$ \\
\hline Std. Dev. (deg.) & $65.64^{\circ}$ & $63.19^{\circ}$ & $61.80^{\circ}$ & $29.62^{\circ}$ & $44.30^{\circ}$ \\
\hline \multicolumn{6}{|l|}{ Range Error } \\
\hline Mean (m.) & $30.65 \mathrm{~m}$ & $30.32 \mathrm{~m}$ & $32.86 \mathrm{~m}$ & $3.58 \mathrm{~m}$ & $6.26 \mathrm{~m}$ \\
\hline Median (m.) & $26.07 \mathrm{~m}$ & $25.58 \mathrm{~m}$ & $26.86 \mathrm{~m}$ & $1.24 \mathrm{~m}$ & $1.64 \mathrm{~m}$ \\
\hline Std. Dev. (m.) & $23.59 \mathrm{~m}$ & $21.89 \mathrm{~m}$ & $23.58 \mathrm{~m}$ & $8.81 \mathrm{~m}$ & $13.86 \mathrm{~m}$ \\
\hline
\end{tabular}


This section shows that not all of the histogram components are necessary for accurate pose estimation. In fact, the histogram may be trimmed to only include $\mathcal{H}=[\cos (\alpha)$, ISOC $]+\sigma$ resulting in a histogram size of $45+104=149$ plus the two cluster scale values $\sigma_{i}$ for the $\mathrm{x}$ - and $y$-axes. A shorter histogram descriptor yields reduced memory storage and a quicker histogram matching procedure. These results validate that comparable pose estimation performance is achievable with a shorter histogram than OUR-CVFH . We offer these results to the user to select their histogram components for scenario and model dependent applications. We maintain the original length of the SOUR-CVFH histogram with all five components plus the addition of two scale values.

\subsubsection{Reference Frame Construction}

The major assumption made by CVFH [79], and carried on by OURCVFH [54], is that the measured point cloud has been segmented such that only one object is in view at a time. Unfortunately, there is no commonly accepted method for 3D scene segmentation — though the process is widely investigated [143, 144, 145]. The segmented scene assumptions permits the use of the OUR-CVFH SGURF, which incorporates the global point-cloud into the construction of the local reference frame (LRF). This semi-global nature of SGURF makes it more repeatable and unique, thus improving pose estimation accuracy and limiting database size. The assumption also proves detrimental in scenes with partially occluded objects because the supporting 

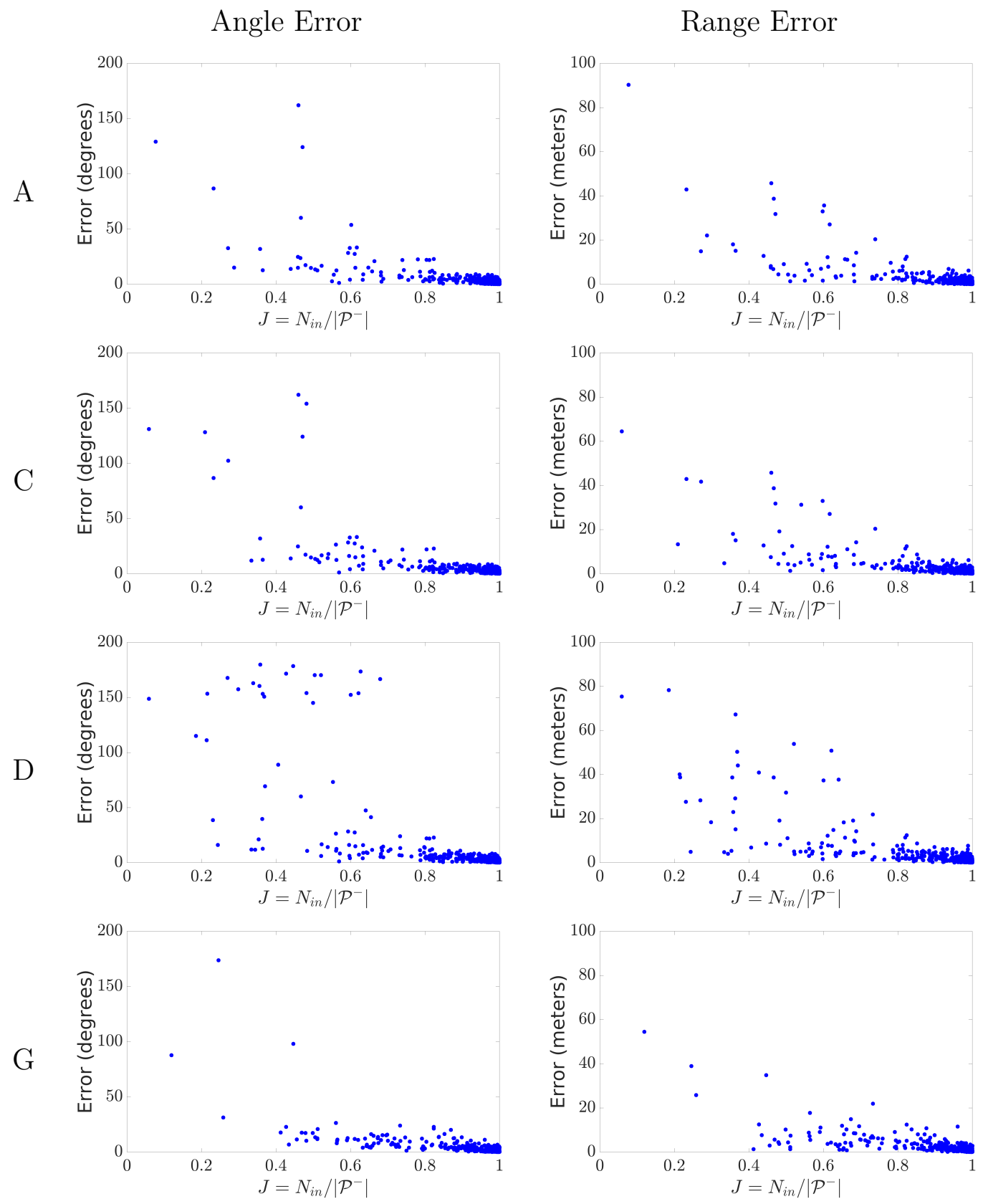

Figure 5.32: All pose estimation errors as a function of cost metric value for the 500 run Monte Carlo varying histogram components for select methods A, C, D, G, I, and U. If the method includes scale, then both scale ratios meet the threshold, $l_{\sigma}=0.9$, and all use distance metric JC. 

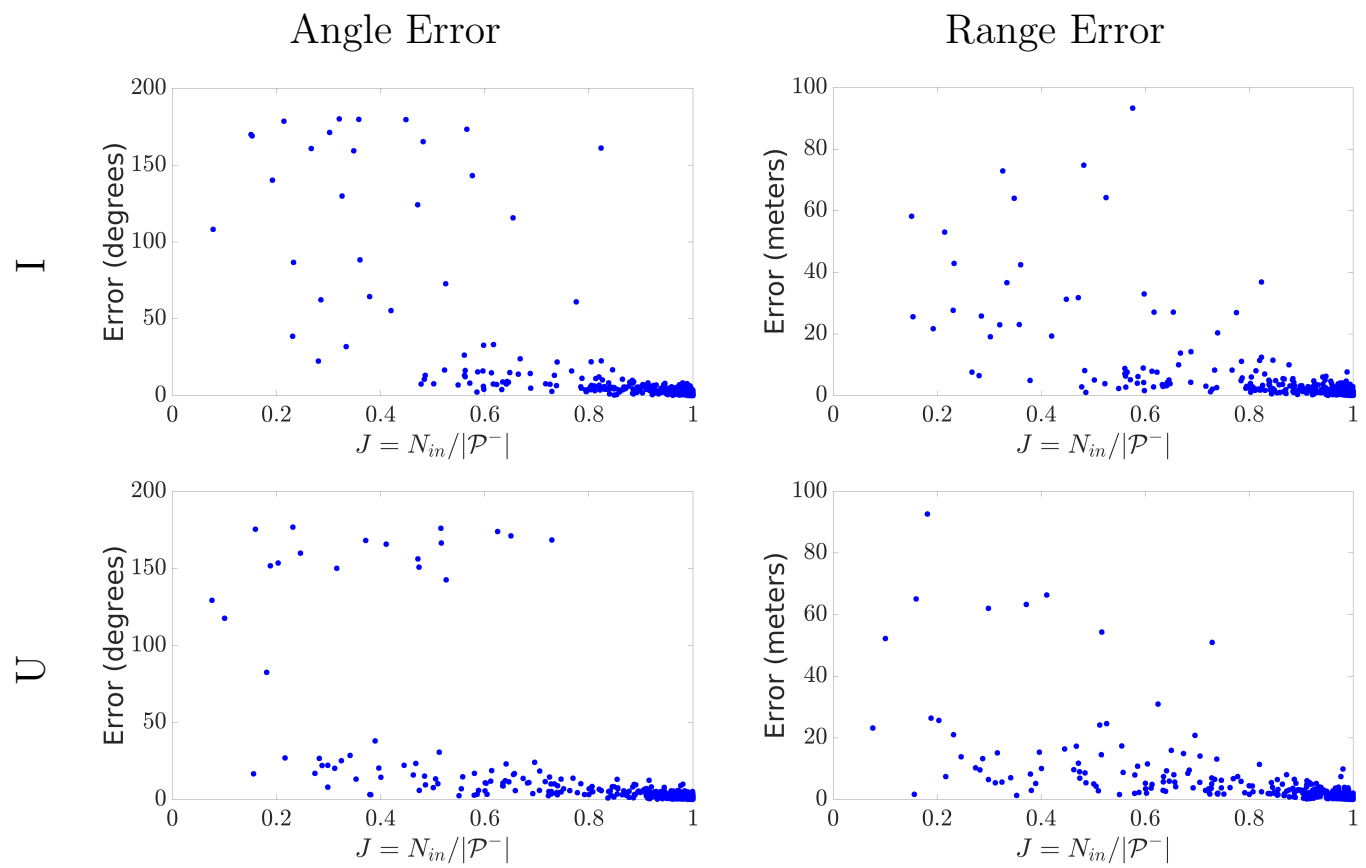

Figure 5.32: Continued. 
global point-cloud now includes surfaces not present in the object training stage.

This section investigates the use of a LRF using a similar construction method as SGURF but only including support from the cluster instead of the entire point-cloud. The LRF will be used for 6DOF pose estimation and to assist in cluster description. If the orientation of the LRF is ambiguous, then multiple (up to four) LRFs will be calculated. This will result in multiple histograms for each cluster. The only change is to Eq. 2.64 where the summation now goes to $m_{\mathbf{p}, k}$ instead of $m_{\mathbf{p}}$. All other modifications up to this point are also included and the ISOC is constructed with LERP.

We call this descriptor SOL-CVFH for Scale and Locally Oriented Clustered Viewpoint Feature Histogram. We feel it is necessary to create a new descriptor name because and SOUR-CVFH heavily depend on SGURF for accuracy and repeatability. Conversion to a LRF substantially changes the performance and theory of the descriptor. What we gain is the ability for pose estimation in non-segmented scenes.

CDF plots of pose estimation results for the 500 run Monte Carlo experiment comparing SOL-CVFH to and SOUR-CVFH is shown in Fig. 5.33. We use a cost metric threshold of $t_{J}=0.5$ for both SOUR-CVFH and SOL-CVFH . SOL-CVFH exhibits performance better than OUR-CVFH, but worse than SOUR-CVFH . Looking at the number of histogram matches in Fig. 5.33c shows that the LRF in SOL-CVFH is less unique than SGURF resulting in more orientations, more histograms per cluster, and thus more histogram 
matches. Using scale as an eliminator before comparing histograms still offers an advantage over OUR-CVFH in reducing the number of histogram matches.

The statistics for the experiment are shown in Table 5.10. While SOLCVFH does offer performance in between OUR-CVFH and SOUR-CVFH, it returns far fewer pose estimates than both OUR-CVFH and SOUR-CVFH . While OUR-CVFH and SOL-CVFH return pose estimates for every scene by construction of the cost metric $J$, using a cost metric threshold is necessary to removing poor estimates.

All pose estimation values as a function of cost metric are shown in Fig. 5.34. The vertical black dotted line is imposed cost metric threshold where values to the left are shown for reference only and not included in the results analysis. SOL-CVFH returns more poor pose estimates with low cost metric values than SOUR-CVFH .

SOL-CVFH exhibits improved performance over OUR-CVFH in terms of pose estimation accuracy and number of histograms matches. It performs worse than OUR-CVFH in terms of the number of estimates returned when the cost metric threshold is set to $t_{J}=0.5$. These results characterize the pure strength of SGURF in setting a unique and repeatable reference frame. In terms of object recognition, or in scenarios where only one object is in view (e.g. space-based applications [16]), SOUR-CVFH would be the preferred method. SOL-CVFH offers a different strength altogether, namely the ability to perform pose estimation in non-segmented scenes (as compared to OURCVFH and SOUR-CVFH ). 


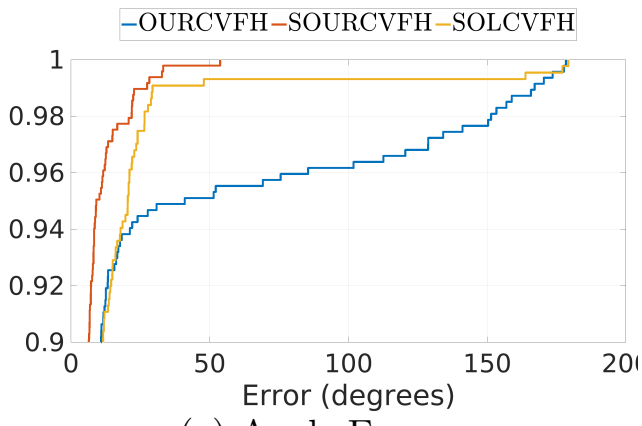

(a) Angle Error

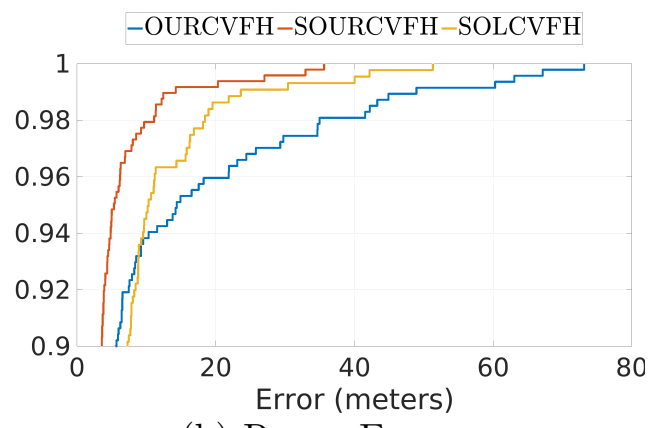

(b) Range Error

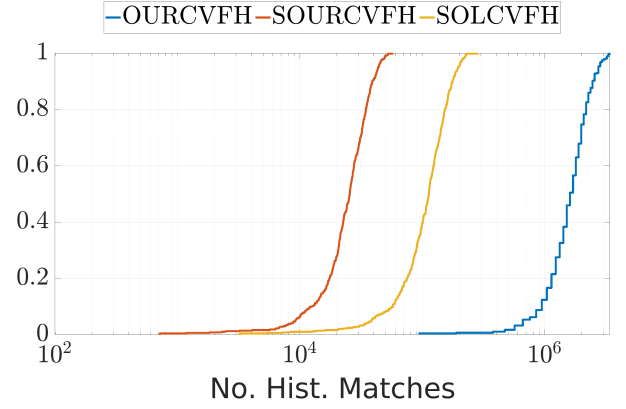

(c) Num. Histogram Matches

Figure 5.33: Pose estimation errors without outliers for 500 run Monte Carlo experiment various ISOC weighting using metric JC and LRF. Methods A, B, and $\mathrm{C}$ use all the modifications up to this point. SOL-CVFH uses a LRF and constructs ISOC with LERP. Cost metric threshold set at $t_{J}=0.5$. 
Table 5.10: Statistics for the 500 run Monte Carlo experiment varying ISOC weighting using metric JC and LRF. SOL-CVFH uses a LRF and constructs ISOC with LERP. Cost metric threshold set at $t_{J}=0.5$.

\begin{tabular}{|c|c|c|c|}
\hline & OUR-CVFH & SOUR-CVFH & SOL-CVFH \\
\hline \multicolumn{4}{|c|}{ Num. Pose Estimates } \\
\hline & 470 & 485 & 437 \\
\hline \multicolumn{4}{|l|}{ Angle Error } \\
\hline Mean (deg.) & $9.78^{\circ}$ & $2.94^{\circ}$ & $5.63^{\circ}$ \\
\hline Median (deg.) & $2.44^{\circ}$ & $1.51^{\circ}$ & $2.31^{\circ}$ \\
\hline Std. Dev. (deg.) & $29.12^{\circ}$ & $4.82^{\circ}$ & $15.21^{\circ}$ \\
\hline \multicolumn{4}{|l|}{ Range Error } \\
\hline Mean (m.) & $3.59 \mathrm{~m}$ & $1.75 \mathrm{~m}$ & $2.82 \mathrm{~m}$ \\
\hline Median (m.) & $1.43 \mathrm{~m}$ & $0.93 \mathrm{~m}$ & $1.23 \mathrm{~m}$ \\
\hline Std. Dev. (m.) & $8.52 \mathrm{~m}$ & $3.17 \mathrm{~m}$ & $5.13 \mathrm{~m}$ \\
\hline \multicolumn{4}{|c|}{ Num. Hist. Matches } \\
\hline Mean & $1.67 e^{6}$ & $2.58 e^{4}$ & $1.14 e^{5}$ \\
\hline Median & $1.60 e^{6}$ & $2.55 e^{4}$ & $1.13 e^{5}$ \\
\hline Std. Dev. & $6.10 e^{5}$ & $1.03 e^{4}$ & $4.67 e^{4}$ \\
\hline
\end{tabular}



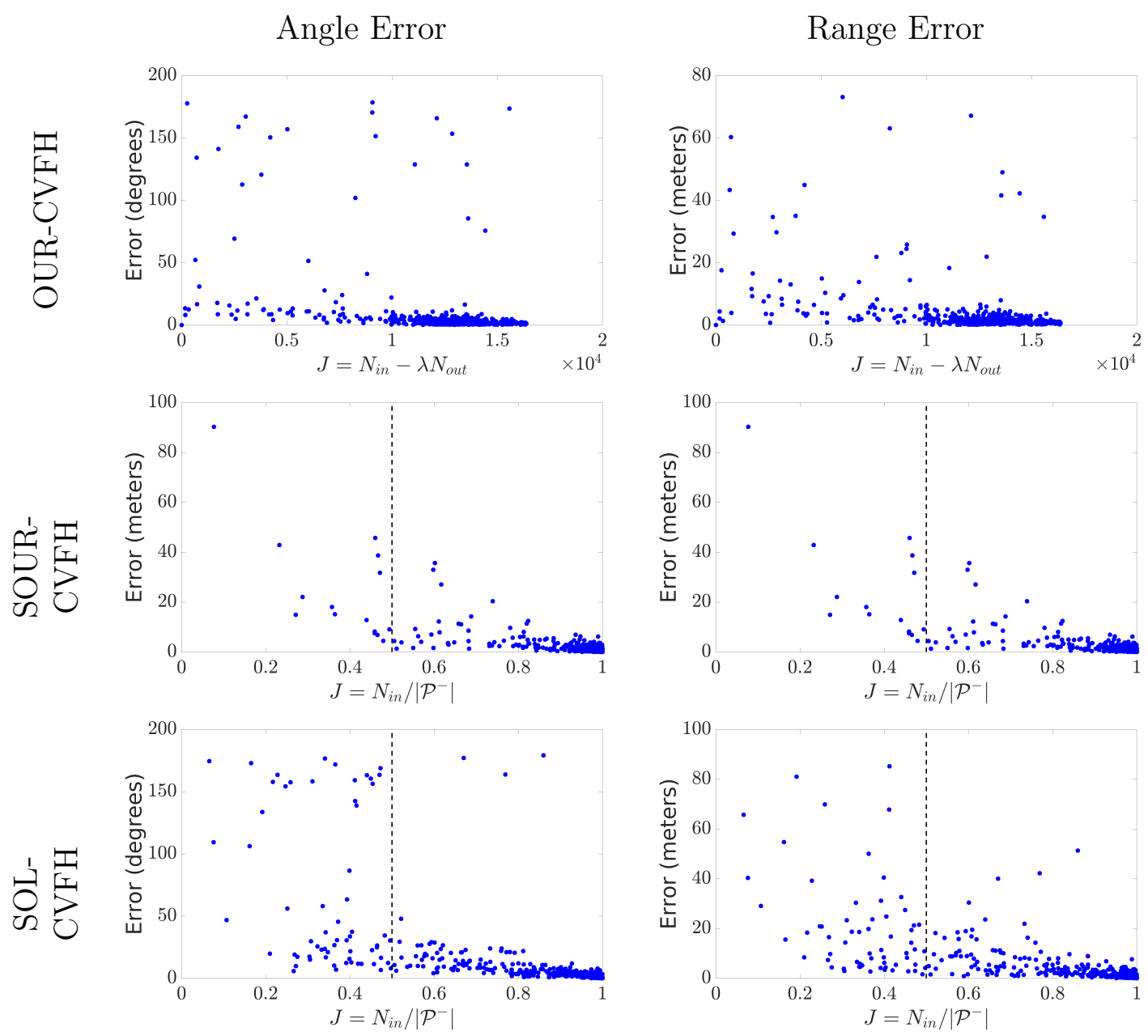

Figure 5.34: All pose estimation errors as a function of cost metric value for 500 run Monte Carlo varying ISOC weighting using metric JC and LRF. SOLCVFH uses a LRF and constructs ISOC with LERP. Vertical black dotted line is cost metric threshold set at $t_{J}=0.5$. 


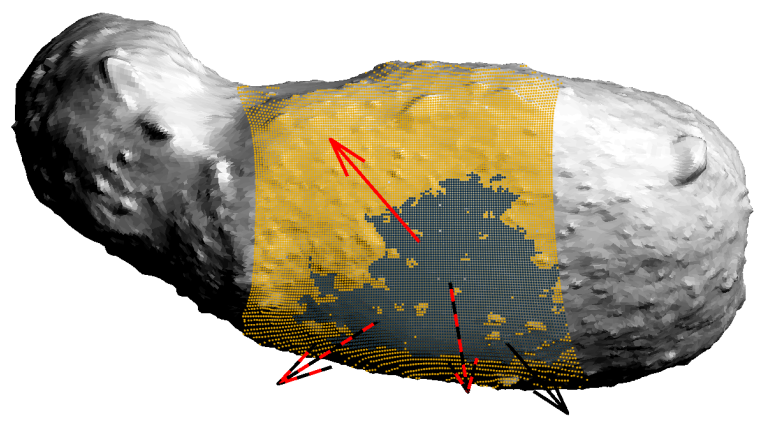

(a) Itokawa with scene, cluster, SGURF, and LRF

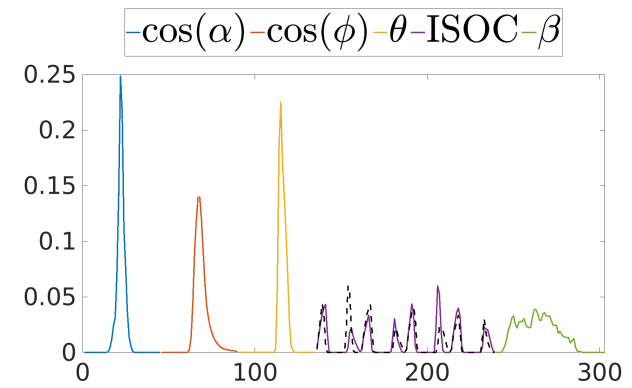

(a) Histograms built with SGURF or LRF

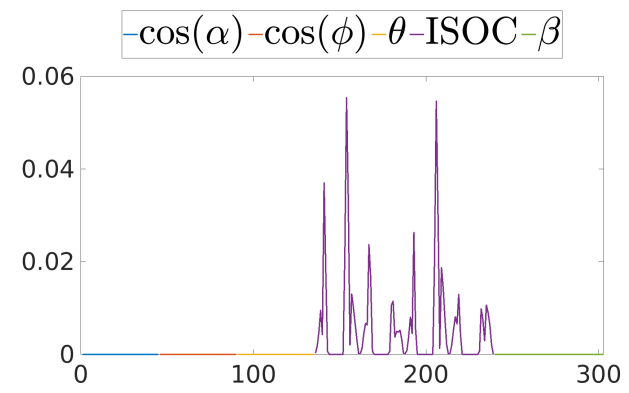

(b) Difference between histograms

Figure 5.35: Visual affects of histogram appearance with SGURF or LRF construction. (a) Itokawa with scene in yellow, cluster in blue, SGURF in red and LRF in black. Notice the only difference between reference frame is a rotation by $90^{\circ}$. (b) Histograms constructed with either SGURF or LRF. (c) Absolute difference between histograms. 


\subsubsection{Comments}

The SOUR-CVFH histogram descriptor is an evolutionary descendant of OUR-CVFH with some important modifications. First, we apply linear interpolation to each of the angular features of $\cos (\alpha), \cos (\phi), \theta$, and $\cos ($ beta $)$ to improve the robustness to sensor noise and measurement artifacts. Second, we modified the description space of $\beta$ to occur in angular space for increased uniqueness. Third, we use the cost metric calculation of Eq. 5.13 such that a pose estimate is returned for every instance. Our method still considers inlier and outlier points, but removes the OUR-CVFH weighting variable $\lambda$. Fourth, we normalize each histogram component to unity to remove the dependency on sensor resolution and for the opportunity of different distance metrics. Fifth, we tested four distance metrics and chose the Jaccard metric of Eq. 5.15. Sixth, we calculate and use the cluster $x$ - and $y$-axes scales $\sigma_{i}$, which is the namesake for SOUR-CVFH . The scale mitigates the quantity of histogram comparisons by only considering clusters of similar size. Previously, OURCVFH considered the unnormalized histogram height as descriptive of the cluster size, but directly using the cluster scale removes any ambiguity. Finally, we modified ISOC weighting scheme to use simply linear interpolation instead of the Gaussian weighting.

Due to the evolutionary development of SOUR-CVFH, it still includes the assumption made by OUR-CVFH of a segmented scene. In instances of clutter, occlusion, or the unavailability of a segmented scene, we developed SOL-CVFH . The main modification is using a LRF instead of SGURF. 
Uniqueness is sacrificed with a LRF leading to a reduction in pose estimation accuracy and an increase in database size and number of histogram matches (as compared to SOUR-CVFH ). Applicability of SOL-CVFH may be more widespread because it removes the segmented scene assumption. SOL-CVFH is better suited to applications that prioritize navigation over object recognition in complicated scenes.

This section methodically describes each modification that was made to OUR-CVFH and the affect it generated. The dissection of each modification is to guide the user towards deeper understanding of SOUR-CVFH (and SOLCVFH ) construction and implementation.

\subsection{Scaled OUR-CVFH (SOUR-CVFH)}

We present the Scaled Oriented Unique Repeatable Clustered Viewpoint Feature Histogram (SOUR-CVFH ). Our descriptor is an extension to OUR-CVFH with specific modifications to intertwine object scale with the histogram construction and as an object identifier. An object's scale is an important inherent characteristic that describes its physical size. Other modifications include (1) a new matching cost metric, (2) a normalized histogram, (3) a new distance metric, (4) a scale comparison step in the recognition framework, and (5) using scale to construct the ISOC histogram.

SOUR-CVFH is able to simultaneously perform object recognition and estimate relative or absolute pose. We continue to use the SGURF of OURCVFH which creates an unambiguous repeatable reference frame for descrip- 
tion and pose estimation. Because SGURF is disambiguated using the entire measured point-cloud, it is required that the scene be segmented into singular objects. We do not address the process of object segmentation, but it is of trivial concern for space based applications where usually only one object of interest is visible.

\subsubsection{Descriptor Construction}

The SOUR-CVFH descriptor contains information regarding the a cluster's shape, size, and orientation. Each of these components play an important role for the object recognition and 6 DOF pose estimation. This section discusses the process of descriptor construction.

\subsubsection{Viewpoint Clustering}

Suppose an explicit surface is measured with a sensor to produce a $3 \mathrm{D}$ point cloud $\mathcal{P}=\left\{\mathbf{p}_{i}, \mathbf{n}_{i}, c_{i}, \epsilon\right\}_{i=1}^{m_{\mathbf{p}}}$ where $\mathbf{p}_{i}$ is a $i$-th $3 \mathrm{D}$ point, $\mathbf{n}_{i}$ is the associated surface normal vector, $c_{i}$ is the associated curvature value, and $\epsilon$ is the spatial resolution found as the median distance between points. Note that $\mathbf{p}_{i}$ is returned by the sensor, while $\mathbf{n}_{i}, c_{i}$, and $\epsilon$ are computed quantities. The same assumption as OUR-CVFH of a segmented scene is still applicable. The point-cloud is first separated into clusters $\mathcal{C}_{k}$ where $k=1, \ldots, m_{\mathcal{C}}$ before describing each one.

1. Remove points from the point-cloud $\mathcal{P}$ if their curvature value $c_{i}$ is greater than a threshold $t_{c}$. The threshold depends on the type of curvature 
used (e.g. Gaussian or mean curvature) and may be a fixed value or set using the interquartile range (IQR). The remaining points and associated surface normal vectors make the filtered point-cloud $\mathcal{P}^{f}=\left\{\mathbf{p}_{i}, \mathbf{n}_{i}\right\}_{i=1}^{m_{\mathbf{p}}^{f}}$, where $m_{\mathbf{p}}^{f} \leq m_{\mathbf{p}}$.

2. The filtered point-cloud $\mathcal{P}^{f}$ is separated into smooth and continuous clusters $\mathcal{C}_{k}$. A cluster is smooth if the inner product of two normal vectors, $\mathbf{n}_{i}$ and $\mathbf{n}_{j}$, is greater than or equal to an angle threshold $t_{\mathbf{n}}$. It is continuous if two points, $\mathbf{p}_{i}$ and $\mathbf{p}_{j}$, are separated by less that an Euclidean distance threshold $t_{d}$. A cluster $\mathcal{C}_{k}$ is initialized with an arbitrary $\mathbf{p}_{i} \in \mathcal{P}^{f}$ that has not yet been assigned to another cluster. Clusters are constructed as

$$
\mathcal{C}_{k}^{\prime}=\left\{\mathbf{p}_{i} \in \mathbf{P}^{f}:\left(\left\|\mathbf{p}_{i}-\mathbf{p}_{j}\right\| \leq t_{d}\right) \wedge\left(\mathbf{n}_{i}^{\top} \mathbf{n}_{j} \leq t_{\mathbf{n}}\right)\right\}_{k=1}^{m_{\mathrm{C}^{\prime}}}
$$

where we set $t_{d}=2.5 \epsilon$ and find that $t_{\mathbf{n}}=\cos \left(2^{\circ}\right)-\cos \left(3^{\circ}\right)$ works well for natural objects with rough surfaces and $t_{\mathbf{n}}=\cos \left(5^{\circ}\right)-\cos \left(7^{\circ}\right)$ works well for objects with mostly flat/smooth surfaces.

3. For each of the $m_{\mathfrak{C}_{k}^{\prime}}$ clusters, calculated the centroid and average normal vector as

$$
\begin{gathered}
\overline{\mathbf{p}}_{k}=\frac{1}{m_{\mathbf{p}, k}^{\prime}} \sum_{i=1}^{m_{\mathbf{p}, k}^{\prime}} \mathbf{p}_{i} \\
\overline{\mathbf{n}}_{k}=\frac{\frac{1}{m_{\mathbf{p}, k}^{\prime}} \sum_{i=1}^{m_{\mathbf{p}, k}^{\prime}} \mathbf{n}_{i}}{\left\|\frac{1}{m_{\mathbf{p}, k}^{\prime}} \sum_{i=1}^{m_{\mathbf{p}, k}^{\prime}} \mathbf{n}_{i}\right\|}
\end{gathered}
$$

where the temporary cluster set is defined as $\mathcal{C}_{k}^{\prime}=\left\{\overline{\mathbf{p}}_{k}, \overline{\mathbf{n}}_{k},\left\{\mathbf{p}_{i}, \mathbf{n}_{i}\right\}_{i=1}^{m_{\mathbf{p}, k}^{\prime}}\right\}_{k=1}^{m_{\mathrm{C}^{\prime}}}$. 
4. Filter once each temporary cluster by the angle between each point's normal vector and the cluster's average normal vector. The resulting clusters satisfy

$$
\mathcal{C}_{k}=\left\{\mathbf{p}_{i} \in \mathcal{C}_{k}^{\prime}:\left(\overline{\mathbf{n}}^{\top} \mathbf{n}_{i} \leq t_{\overline{\mathbf{n}}}\right)\right\}_{k=1}^{m_{\mathcal{C}}}
$$

where we set $t_{\overline{\mathbf{n}}}=t_{\mathbf{n}} / 10$ in order to stay variable with the previous defintion of $t_{\mathbf{n}}$. The final cluster set is $\mathcal{C}_{k}=\left\{\overline{\mathbf{p}}_{k}, \overline{\mathbf{n}}_{k},\left\{\mathbf{p}_{i}, \mathbf{n}_{i}\right\}_{i=1}^{m_{\mathbf{p}, k}}\right\}_{k=1}^{m_{e}}$ where $m_{\mathbf{p}, k} \leq m_{\mathbf{p}, k}^{\prime}$. Note that the cluster centroid and average normal vector are not recalculated.

5. A cluster is kept only if its quantity of points $m_{\mathbf{p}, k}$ is more than a threshold $t_{m_{\mathbf{p}}}$. We set the threshold as $t_{m_{\mathbf{p}}}=50$. If no clusters are found, then the entire point-cloud is considered a cluster and sent through the process described here.

\subsubsection{Semi-Global Unique Reference Frame (SGURF) and Ob- ject Scale}

A SGURF is calculated for each cluster and fixed at the cluster centroid $\mathbf{p}_{k}$. It is used to directly find the $6 \mathrm{DOF}$ relative pose and to assist in cluster description. A SGURF's orientation depends on the spatial distribution of points in the whole scene, not just the cluster. If the orientation is ambiguous, multiple SGURFs (up to four) will be calculated, resulting in multiple SOURCVFH histograms for each cluster. Calculation of the SGURF is identical to that of OUR-CVFH [54]. 
1. For each cluster, compute the scatter matrix $[54,88]$

$$
\mathbf{M}=\frac{1}{\sum_{i=1}^{m_{\mathbf{p}, k}}\left(d_{\max }-d_{i}\right)} \sum_{i=1}^{m_{\mathbf{p}, k}}\left(d_{\max }-d_{i}\right)\left(\mathbf{p}_{i}-\overline{\mathbf{p}}_{k}\right)\left(\mathbf{p}_{i}-\overline{\mathbf{p}}_{k}\right)^{\top}
$$

where $d_{i}=\left\|\mathbf{p}_{i}-\overline{\mathbf{p}}_{k}\right\|$ and $d_{\max }=\max _{\mathbf{p}_{i} \in \mathcal{C}_{k}}\left\|\mathbf{p}_{i}-\overline{\mathbf{p}}_{k}\right\|$. The weighting scheme of this scatter matrix deprioritizes points far from the centroid that are likely to be occluded in sensor measurements.

2. Compute the eigen-decomposition of the scatter matrix $\mathbf{M}$ to find the eigenvectors $\mathbf{e}_{1}, \mathbf{e}_{2}, \mathbf{e}_{3}$, and the corresponding eigenvalues $\lambda_{1}, \lambda_{2}, \lambda_{3}$ given in descending order of value.

3. The $z$-axis is selected as the eigenvector with the smallest eigenvalue, usually considered $\lambda_{3}$ and $\mathbf{e}_{3}$ because eigenvalues are commonly listed in descending order. The $z$-axis points in the same direction as the cluster average normal vector, towards the sensor. The direction of the $z$-axis is disambiguated as

$$
\mathbf{z}= \begin{cases}\mathbf{e}_{3} & \text { if } \overline{\mathbf{n}}_{k}^{\top} \mathbf{e}_{3}>0 \\ -\mathbf{e}_{3} & \text { otherwise }\end{cases}
$$

4. While SGURF is locally defined by the cluster, the $x$ - and $y$-axis are disambiguated globally using the entire point-cloud $\mathcal{P}$. Temporarily rename the remaining eigenvectors $\mathbf{e}_{1}^{+}, \mathbf{e}_{2}^{+}$, and their opposites $\mathbf{e}_{1}^{-}, \mathbf{e}_{2}^{-}$. The objective is to orient the axes towards the most populated hemispheres defined by the eigenvectors. Calculate the point densities for $\mathbf{e}_{1}$ as

$$
\Psi_{\mathbf{e}_{1}}^{ \pm}=\sum_{i=1}^{m_{\mathbf{p}}} \begin{cases}\left|\psi_{i}^{ \pm}\right| \psi_{i}^{ \pm} & \text {if } \psi_{i}^{ \pm} \geq 0 \\ 0 & \text { otherwise }\end{cases}
$$


where

$$
\psi_{i}^{ \pm}=\left(\mathbf{p}_{i}-\overline{\mathbf{p}}_{k}\right)^{\top} \mathbf{e}_{1}^{ \pm}
$$

and similarly for $\mathbf{e}_{2}$. The sign of $\mathbf{e}_{1}$ is disambiguated as

$$
\mathbf{e}_{1}= \begin{cases}\mathbf{e}_{1}^{+} & \text {if }\left|\Psi_{\mathbf{e}_{1}}^{+}\right| \geq\left|\Psi_{\mathbf{e}_{1}}^{-}\right| \\ \mathbf{e}_{1}^{-} & \text {otherwise }\end{cases}
$$

where the sign of $\mathbf{e}_{2}$ is disambiguated similarly.

5. Calculate the axes disambiguation factors $\mathrm{f}_{1}, \mathrm{f}_{2}$ as

$$
\mathbf{f}_{j}=\frac{\min \left(\left|\Psi_{\mathbf{e}_{j}}^{+}\right|,\left|\Psi_{\mathbf{e}_{j}}^{-}\right|\right)}{\max \left(\left|\Psi_{\mathbf{e}_{j}}^{+}\right|,\left|\Psi_{\mathbf{e}_{j}}^{-}\right|\right)}, \quad j=1,2
$$

where the factor is bounded by $f \in[0,1], f=0$ is complete disambiguation, and $f=1$ is complete ambiguity.

6. The $x$-axis is selected as the eigenvector with the lowest disambiguation factor $f$

$$
\mathbf{x}= \begin{cases}\mathbf{e}_{1} & \text { if } \mathrm{f}_{1}<\mathrm{f}_{2} \\ \mathbf{e}_{2} & \text { otherwise }\end{cases}
$$

and the $y$-axis is calculated as $\mathbf{y}=\mathbf{z} \times \mathbf{x}$ to create a right-handed system.

7. Occasionally, the axis disambiguation is not robust. If both disambiguation factors are similar in value, then two SGURFs are created. Calculate the factor ratio as

$$
r_{\mathrm{f}}=\frac{\min \left(\mathrm{f}_{1}, \mathrm{f}_{2}\right)}{\max \left(\mathrm{f}_{1}, \mathrm{f}_{2}\right)}
$$

If $\mathrm{r}_{\mathrm{f}}>t_{\mathrm{r}_{\mathrm{f}}}$, then create two SGURFs, one with $\mathbf{e}_{1}$ as the $x$-axis and the other with $\mathbf{e}_{2}$ as the $x$-axis. This threshold is set as $t_{\mathrm{r}_{\mathrm{f}}}=0.8$. Additionally, if the smaller disambiguation factor is greater than a threshold 
$t_{\mathrm{f}}$, then there is absolute ambiguity of the cluster orientation and four SGURFs are generated — one for each sign of each eigenvector as the $x$-axis. This threshold is set as $t_{\mathrm{f}}=0.925$.

Through the process of defining a cluster's SGURF, we may simultaneously define its scale. Three scales are attributed to each cluster, one for each SGURF axis. Scale values assist in cluster description through the histogram, and to make the recognition framework more efficient.

1. Maintain the eigenvalue and eigenvector associations while disambiguating the SGURF axes. Since the clustering routing of Sec. 5.3.1.1 prioritizes smooth clusters (i.e. nearly planar), we define the axes scales as

$$
\sigma_{i}=3 \sqrt{\lambda_{i}}, \quad i=1,2,3
$$

where the support window of 3 offers $98.8 \%$ coverage of the cluster (in a 2D sense). These scales may be different for each axis. If multiple SGURFs are constructed for a cluster, the scales remain constant in value, but are reordered to correspond to the SGURF axes.

Before using the SGURF for pose estimation or in constructing the histogram, we must find the transformation from the sensor frame $S$ to the SGURF frame denoted as $F$. The transformation is found with with following procedure. 
1. Calculate the viewpoint vector from the cluster centroid to the sensor origin as

$$
\mathbf{v}=\frac{\mathbf{s}_{o}-\overline{\mathbf{p}}_{k}}{\left\|\mathbf{s}_{o}-\overline{\mathbf{p}}_{k}\right\|}
$$

where $s_{o}$ is the sensor origin commonly assumed to be $\mathbf{s}_{o}=[0,0,0]^{\top}$.

2. Take the cross product of the viewpoint vector with the sensor boresight as

$$
\mathbf{c}=\mathbf{v} \times \mathbf{s}_{b}
$$

where the sensor boresight is commonly assumed to be $\mathbf{s}_{b}=[0,0,1]^{\top}$.

3. Convert the vector $\mathbf{c}$ to Rodrigues parameters in terms of the Euler axis and angle as

$$
\begin{aligned}
\psi & =\arcsin (\|\mathbf{c}\|) \\
\mathbf{e}_{\psi} & =\frac{\mathbf{c}}{\|\mathbf{c}\|}
\end{aligned}
$$

where $\psi$ is the rotation angle and $\mathbf{e}_{\psi}$ is the axis of rotation.

4. Use Rodrigues' rotation formula to convert the Rodrigues parameters to a rotation matrix $\mathbf{R}_{I}^{S}$ from the sensors frame $S$ to an intermediate frame $I$ that lies along the sensor boresight

$$
\mathbf{R}_{I}^{S}=\cos (\psi) \mathbf{I}_{3 \times 3}+(1-\cos (\psi)) \mathbf{e}_{\psi} \mathbf{e}_{\psi}^{\top}-\sin (\psi)\left[\mathbf{e}_{\psi} \times\right]
$$

where $\mathbf{I}$ is the $3 \times 3$ identity matrix and $[\cdot \times]$ is the skew-symmetric matrix. 
5. Recall that the SGURF is the local coordinate frame fixed at the cluster centroid. It may also be viewed as a frame transformation $\mathbf{R}_{I}^{F}$ from the SGURF frame $F$ to the intermediate frame $I$. Rotation and translation of points in the sensor frame to the SGURF frame is accomplished using

$$
\begin{aligned}
\mathbf{R}_{F}^{S} & =\mathbf{R}_{F}^{I} \mathbf{R}_{I}^{S} \\
\mathbf{t}_{F} & =-\mathbf{R}_{F}^{I} \mathbf{R}_{I}^{S} \overline{\mathbf{p}}_{S, k}
\end{aligned}
$$

where $\mathbf{R}_{F}^{S}$ is the rotation from the sensor frame $S$ to the SGURF frame $F$, and $t_{F}$ is the negated translation in the SGURF frame. Both the rotation and translation may be applied to points simultaneously using a homogeneous transformation. The transformation $\mathbf{T}_{F}^{S}$ from the sensor frame $S$ to the local SGURF frame $F$ is defined as

$$
\mathbf{T}_{F}^{S}=\left[\begin{array}{cc}
\mathbf{R}_{F}^{S} & \mathbf{t}_{F} \\
\mathbf{0}_{1 \times 3} & 1
\end{array}\right]
$$

This transformation is used in the spatial description of the cluster as well as in pose estimation.

\subsubsection{Histogram Construction}

A five-component histogram of concatenated spatial and angular distributions of points and normal vectors is used to concisely describe each cluster. Each cluster will have at least one histogram, with a possibility of a maximum of four — one histogram per SGURF. Angular distributions are calculated using the cluster average normal vector and a local reference frame for each 
point. The spatial distribution describes the point locations defined by the SGURF axes that we call the Interpolated Spatial Octant Component (ISOC). Angular distributions remain constant, while the ISOC changes per SGURF. The complete histogram contains 303 bins. Histogram components are found as follows.

1. For every point $\mathbf{p}_{i} \in \mathfrak{C}_{k}$, define the Darboux coordinate frame

$$
\mathrm{D}=(\mathbf{u}, \mathbf{v}, \mathbf{w}) \text { as [78] }
$$

$$
\begin{aligned}
\mathbf{u}_{i} & =\overline{\mathbf{n}}_{k} \\
\mathbf{v}_{i} & =\frac{\left(\mathbf{p}_{i}-\overline{\mathbf{p}}_{k}\right) \times \mathbf{u}_{i}}{\left\|\left(\mathbf{p}_{i}-\overline{\mathbf{p}}_{k}\right) \times \mathbf{u}_{i}\right\|} \\
\mathbf{w}_{i} & =\mathbf{u}_{i} \times \mathbf{v}_{i}
\end{aligned}
$$

2. Calculate the angular distributions of the histogram

$$
\begin{aligned}
\cos \left(\alpha_{i}\right) & =\mathbf{v}_{i}^{\top} \mathbf{n}_{i} \\
\cos \left(\phi_{i}\right) & =\mathbf{u}_{i}^{\top}\left(\frac{\mathbf{p}_{i}-\overline{\mathbf{p}}_{k}}{\left\|\mathbf{p}_{i}-\overline{\mathbf{p}}_{k}\right\|}\right) \\
\theta_{i} & =\arctan \left(\frac{\mathbf{w}_{i}^{\top} \mathbf{n}_{i}}{\mathbf{u}_{i}^{\top} \mathbf{n}_{i}}\right) \\
\beta_{i} & =\arccos \left(\mathbf{n}_{i}^{\top} \mathbf{v}\right)
\end{aligned}
$$

where $\mathbf{v}$ in Eq. 5.49 is from Eq. 5.35. Based on the physical constraints of the scene, each of these components is reasonable bounded. Components $\cos \left(\alpha_{i}\right)$ and $\cos \left(\phi_{i}\right)$ exist within the range \pm 1 . The $\theta_{i}$ component exists within the range $\pm 90^{\circ}$ meaning the bins are in $4^{\circ}$ increments. Since the surface normal vectors always point toward the sensor, $\beta_{i}$ is bounded by $\left[0^{\circ}, 90^{\circ}\right]$ with bin increments of $1.406^{\circ}$. 
3. Perform linear interpolation between neighboring bins with the angular distribution values. The weights are accumulated as

$$
b_{\gamma, i}=\sum_{i \in w_{b}} 1-d_{\gamma, i}, \quad \gamma=\alpha, \phi, \theta, \beta
$$

where $\gamma$ is cycling through the distributions, $d_{\gamma, i} \leq 1$ is the distance from the angular value to the bin center, and the weight is distributed across a maximum of two bins. Note that $\cos \left(\alpha_{i}\right)$ is the first component with $w_{b}=45$ bins, $\cos \left(\phi_{i}\right)$ is the second component with $w_{b}=45$ bins, $\theta_{i}$ is the third component with $w_{b}=45$ bins, and $\beta_{i}$ is the fifth component with $w_{b}=64$ bins.

4. Calculate the spatial distribution of the histogram using the points in $\mathcal{C}_{k}$. Use the transformation of Eq. 5.42 to transform the cluster $\mathcal{C}_{k}$ from the sensor frame $S$ to the local SGURF frame $F$. Cluster points are now naturally divided into frame's octants described by the signed axes $\left(x^{ \pm}, y^{ \pm}, z^{ \pm}\right)$

5. Consideration of SGURF axes variation and sensor noise is accomplished by inter- and intra-octant interpolation of point locations. Each octant is separated into 13 bins dependent on a point's normalized distance from the origin. Calculated the spatial distribution of points as

$$
\Delta_{\eta, i}=\frac{12\left\|\mathbf{p}_{\eta, i}-\overline{\mathbf{p}}_{\eta, k}\right\|}{\max _{\mathbf{p}_{i} \in \mathcal{C}_{k}}\left\|\mathbf{p}_{\eta, i}-\overline{\mathbf{p}}_{\eta, k}\right\|}, \quad \eta=x, y, z
$$

where $\eta$ is cycling through the axes, and $\Delta_{i} \in[0,12]$ for each octant which is thirteen elements long. 

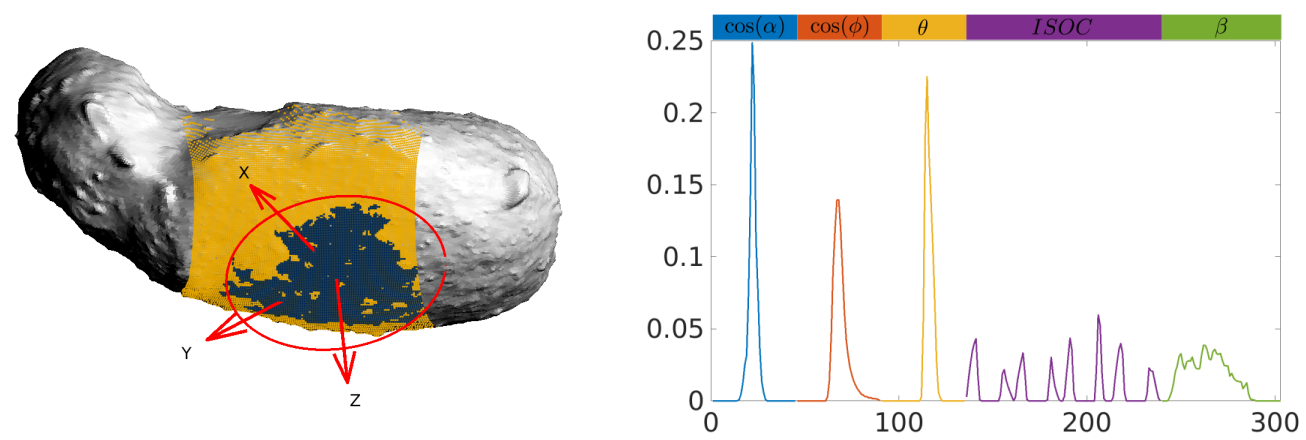

Figure 5.36: SOUR-CVFH cluster and histogram example. (left) Itokawa with the scene in yellow, cluster in blue, with 2D bounding circle and axes in red. (right) SOUR-CVFH histogram with labeled components.

6. Perform linear interpolation between neighboring bins with the spatial distribution values. The weights are accumulated as

$$
b_{\eta, i}=\sum_{i \in \eta} 1-d_{\eta, i}
$$

where $d_{\eta, i}$ is the distance from $\Delta_{\eta, i}$ to the bin center, and the weight is distributed across a maximum of six bins. Note that $\Delta_{\eta, i}$ is the fourth histogram component. This scheme produces a ISOC size of 104 bins (i.e. 8 octants with 13 bins each).

7. The complete SOUR-CVFH histogram contains 303 bins combined in the order of $\mathcal{H}=[\cos (\alpha), \cos (\phi), \theta$, ISOC, $\beta]$. Recall that each cluster will have the same number of histograms as it does unique SGURFs with a maximum possibility of four. An example of a scene with cluster and SOUR-CVFH histogram is shown in Fig. 5.36. 

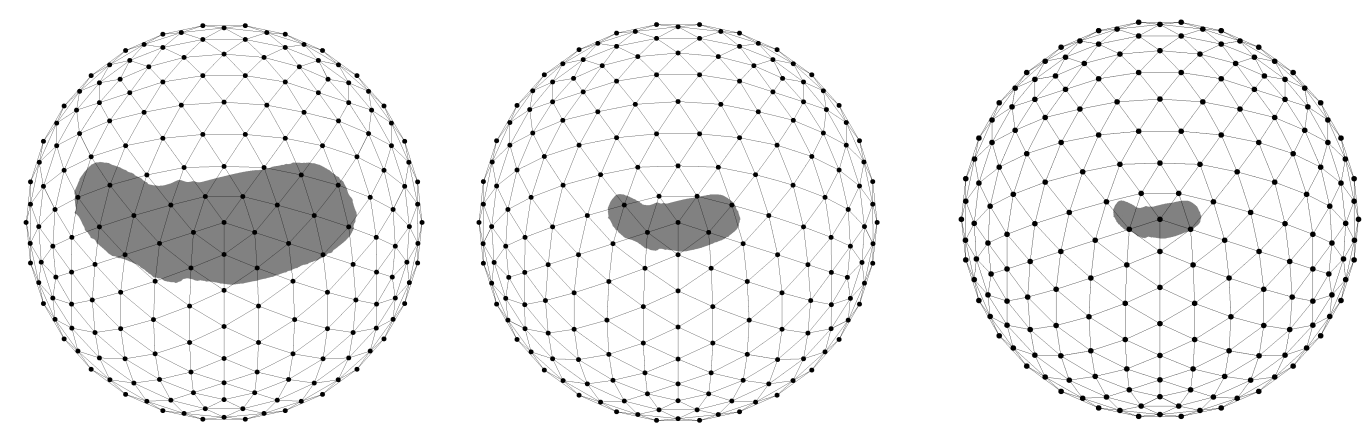

Figure 5.37: Example locations and ranges for database training procedure. The camera is placed at the vertices of a polyhedron oriented towards the object at multiple ranges.

\subsubsection{Training Database Construction}

Using SOUR-CVFH or SOL-CVFH for applications of object recognition requires a training procedure. Training is performed using a simulated 3D sensor and a virtual 3D model if available. Otherwise, training is performed by first circumnavigating the object while taking measurements at a variety of ranges. The final measurement must be a 3D point-cloud, which is provided by a variety of 3D sensors such as LIDAR [7], stereoscopic systems, structured light sensors $[5,6]$, or spacetime stereo [8, 9]. Open source LIDAR simulators are available too, such as GLIDAR [13], but we use an in-house LIDAR simulator built in MATLAB.

Training is performed for a variety of ranges and orientations. Place the object of interest at the center of a polyhedron with equally spaced vertices. The quantity of vertices is application dependent. The polyhedron is constructed for each specified training range. Ranges are discrete samples of the 
intended operating range. This method of training ensures that the database has measured the object at a variety or attitudes and ranges sufficient for object recognition. An example of an object placed at the center of a polyhedron at multiple ranges is shown in Fig. 5.37.

SOUR-CVFH and SOL-CVFH provide relative pose between the model's original pose and its pose in the scene. If the application requires relative pose from the training sensor to the active sensor, then an additional pose must be stored. The sensor pose at each of the polyhedron vertices at each range is stored as $\mathbf{T}_{S}^{B}$.

If the pose relative the camera is required, then an additional pose

\subsubsection{Recognition Framework}

1. Compare the scales of every scene cluster $\widetilde{\mathcal{C}}_{k} \in \widetilde{\mathcal{S}}$, to the scales of every model cluster $\mathcal{C}_{k} \in \mathcal{M}$ as

$$
\mathrm{r}_{\sigma, j}=\frac{\min \left(\sigma_{\widetilde{\mathcal{S}}, j}, \sigma_{\mathcal{M}, j}\right)}{\max \left(\sigma_{\widetilde{\mathcal{S}}, j}, \sigma_{\mathcal{M}, j}\right)}, \quad j=1,2
$$

where $\mathbf{r}_{\sigma}$ is bounded by $\mathbf{r}_{\sigma} \in[0,1]$. We compare scales from corresponding axes of the scene and model clusters because this would be the assigned relative pose from the SGURFs. If both scale ratios are greater than the threshold $t_{\mathrm{r}_{\sigma}}$, then the scene and model clusters are of similar scale and histogram matching may proceed. We experimentally set $t_{\mathrm{r}_{\sigma}}=0.9$.

2. Match the scene cluster's histogram $\widetilde{\mathcal{H}} \in \widetilde{\mathcal{S}}$ to the model cluster's histogram $\mathcal{H} \in \mathcal{M}$ using the Jaccard distance metric 


$$
\mathrm{d}_{\mathrm{JC}}(\widetilde{\mathcal{H}}, \mathcal{H})=1-\frac{\sum_{i=1}^{303} \min \left(\widetilde{\mathcal{H}}_{i}, \mathcal{H}_{i}\right)}{\sum_{i=1}^{303} \max \left(\widetilde{\mathcal{H}}_{i}, \mathcal{H}_{i}\right)}
$$

3. Take the best $m_{d}$ matches with the smallest distance metric. We select $m_{d}=10$. Find the relative 6 DOF pose of the initial ICP alignment as

$$
\delta \widetilde{\mathbf{T}}_{S}^{-}=\widetilde{\mathbf{T}}_{S}^{F} \mathbf{T}_{F}^{S}
$$

where $\delta \mathbf{T}_{S}$ is the relative pose between training and sensed SGURF poses and the $(-)$ superscript denotes before ICP improvement.

4. Use ICP to refine the relative pose using $\delta \widetilde{\mathbf{T}}_{S}^{-}$as an initial alignment. The relative pose between the model object and the measured scene is give by the ICP output refined pose $\delta \widetilde{\mathbf{T}}_{S}^{+}$. This is the relative pose from the inertial setting.

5. Find the final absolute 6 DOF pose from the object body $B$ to the sensor $S$ as

$$
\widetilde{\mathbf{T}}_{S}^{B}=\delta \widetilde{\mathbf{T}}_{S}^{+} \mathbf{T}_{S}^{B}
$$

where $\mathbf{T}_{S}^{B} \in \mathcal{M}$ is the true training pose from the object body $B$ to the sensor $S$.

6. For each of the $m_{d}$ matches, use $\widetilde{\mathbf{T}}_{S}^{B}$ to transform the training and sensed point-clouds into the same reference frame. Back project the model point-cloud onto the scene camera. Model points not visible by the 
scene camera are occluded, so remove them from consideration. Each remaining model point is considered an inlier if it lies within a distance threshold $t_{d}=2 \epsilon$, else it is an outlier. Calculate the cost metric

$$
J=\frac{m_{i n}}{|\mathcal{P}-|}
$$

where

$$
\left|\mathcal{P}^{-}\right|=|\mathcal{P}|-m_{\text {occlude }}=m_{\text {in }}+m_{\text {out }}
$$

$|\cdot|$ returns the set cardinality, and $m_{\text {occlude }}, m_{\text {in }}$, and $m_{\text {out }}$ are the occluded, inlier, and outlier model points respectively. The recognition framework returns the pose estimate that maximizes the Eq. 5.57 so long as $J>t_{J}$. We experimentally set $t_{J}=0.5$. If the cost metric threshold is not met, then no pose estimate is returned by the recognition framework.

Comparing cluster scale before calculating histogram distances reduces poor pose estimates and quickens runtime by ignoring improbably matches. Step 1 of this section suggests comparing every combination of scales. More efficient methods of searching are available such as a binary search tree (BST) or kd-tree.

\subsubsection{Comments on SOUR-CVFH}

The SOUR-CVFH descriptor is less affected by point-cloud resolution than OUR-CVFH because its histogram is normalized. SOUR-CVFH also is 
less affected by noise because all of the histogram components are interpolated. While we do not preform the experiments here, we believe this has important applications to collaborative multi-robotic environments. Suppose each robot is equipped with a 3D sensor of different physical attributes. The SOUR-CVFH descriptor allows each of these robots to share the same training database and measured scenes without affecting recognition and pose estimation. This means that the many robots need not be of the same model, but may be constructed piecemeal over model generations. 
Table 5.11: Threshold variables, preset values, and their applicable equation for SOUR-CVFH .

\begin{tabular}{llll}
\hline \hline Threshold & Description & Value & Applicable Equation \\
\hline$t_{c}$ & curvature & (method dependent) & Sec. 5.3.1.1 Step 1 \\
$t_{d}$ & clustering distance & $2.5 \epsilon$ & Eq. 5.22 \\
$t_{\mathbf{n}}$ & clustering angle & $\cos \left(2^{\circ}\right)-\cos \left(3^{\circ}\right)$ & Eq. 5.22 \\
& & $\cos \left(5^{\circ}\right)-\cos \left(7^{\circ}\right)$ & \\
$t_{\overline{\mathbf{n}}}$ & re-clustering angle & $t_{\mathbf{n}} / 10$ & Eq. 5.25 \\
$t_{m_{\mathbf{p}}}$ & minimum cluster size & 50 & Eq. 5.25 \\
$t_{r_{\mathrm{f}}}$ & disambiguation factor ratio & 0.8 & Eq. 5.64 \\
$t_{\mathrm{f}}$ & disambiguation factor ratio & 0.925 & Eq. 5.64 \\
$t_{\mathrm{r}_{\sigma}}$ & scale ratio threshold & 0.9 & Eq. 5.53 \\
$t_{d}$ & point-cloud inlier / outlier & $2 \epsilon$ & Eq. 5.58 \\
$t_{J}$ & cost metric & 0.5 & Eq. 5.57 \\
\hline \hline
\end{tabular}




\subsection{Scaled and Oriented Locally CVFH (SOL-CVFH) 5.4.1 Local Reference Frame (LRF) and Object Scale}

A LRF is calculated for each cluster and fixed at the cluster centroid $\mathbf{p}_{k}$. It is used to directly find the $6 \mathrm{DOF}$ relative pose and to assist in cluster description. In contrast to SOUR-CVFH, the LRF orientation for SOLCVFH depends on the spatial distribution of points in the clusters. If the orientation is ambiguous, multiple LEFs (up to four) will be calculated, resulting in multiple SOL-CVFH histograms for each cluster. Calculation of the LRF is identical to that of SOUR-CVFH with the exception of Eq. 5.28. This discussion presents the procedure for constructing the SOL-CVFH LRF. Notice the difference in Eq. 5.61.

1. For each cluster, compute the scatter matrix $[54,88]$

$$
\mathbf{M}=\frac{1}{\sum_{i=1}^{m_{\mathbf{p}, k}}\left(d_{\max }-d_{i}\right)} \sum_{i=1}^{m_{\mathbf{p}, k}}\left(d_{\max }-d_{i}\right)\left(\mathbf{p}_{i}-\overline{\mathbf{p}}_{k}\right)\left(\mathbf{p}_{i}-\overline{\mathbf{p}}_{k}\right)^{\top}
$$

where $d_{i}=\left\|\mathbf{p}_{i}-\overline{\mathbf{p}}_{k}\right\|$ and $d_{\max }=\max _{\mathbf{p}_{i} \in \mathcal{C}_{k}}\left\|\mathbf{p}_{i}-\overline{\mathbf{p}}_{k}\right\|$. The weighting scheme of this scatter matrix deprioritizes points far from the centroid that are likely to be occluded in sensor measurements.

2. Compute the eigen-decomposition of the scatter matrix $\mathbf{M}$ to find the eigenvectors $\mathbf{e}_{1}, \mathbf{e}_{2}, \mathbf{e}_{3}$, and the corresponding eigenvalues $\lambda_{1}, \lambda_{2}, \lambda_{3}$ given in descending order of value.

3. The $z$-axis is selected as the eigenvector with the smallest eigenvalue, usually considered $\lambda_{3}$ and $\mathbf{e}_{3}$ because eigenvalues are commonly listed in 
descending order. The $z$-axis points in the same direction as the cluster average normal vector, towards the sensor. The direction of the $z$-axis is disambiguated as

$$
\mathbf{z}= \begin{cases}\mathbf{e}_{3} & \text { if } \overline{\mathbf{n}}_{k}^{\top} \mathbf{e}_{3}>0 \\ -\mathbf{e}_{3} & \text { otherwise }\end{cases}
$$

4. The LRF is disambiguated by the distribution of points within the cluster $\mathcal{C}_{k}$. Temporarily rename the remaining eigenvectors $\mathbf{e}_{1}^{+}, \mathbf{e}_{2}^{+}$, and their opposites $\mathbf{e}_{1}^{-}, \mathbf{e}_{2}^{-}$. The objective is to orient the axes towards the most populated hemispheres defined by the eigenvectors. Calculate the point densities for $\mathbf{e}_{1}$ as

$$
\Psi_{\mathbf{e}_{1}}^{ \pm}=\sum_{i=1}^{m_{\mathbf{p}, k}} \begin{cases}\left|\psi_{i}^{ \pm}\right| \psi_{i}^{ \pm} & \text {if } \psi_{i}^{ \pm} \geq 0 \\ 0 & \text { otherwise }\end{cases}
$$

where

$$
\psi_{i}^{ \pm}=\left(\mathbf{p}_{i}-\overline{\mathbf{p}}_{k}\right)^{\top} \mathbf{e}_{1}^{ \pm}
$$

and similarly for $\mathbf{e}_{2}$. The sign of $\mathbf{e}_{1}$ is disambiguated as

$$
\mathbf{e}_{1}= \begin{cases}\mathbf{e}_{1}^{+} & \text {if }\left|\Psi_{\mathbf{e}_{1}}^{+}\right| \geq\left|\Psi_{\mathbf{e}_{1}}^{-}\right| \\ \mathbf{e}_{1}^{-} & \text {otherwise }\end{cases}
$$

where the sign of $\mathbf{e}_{2}$ is disambiguated similarly.

5. Calculate the axes disambiguation factors $f_{1}, f_{2}$ as

$$
\mathrm{f}_{j}=\frac{\min \left(\left|\Psi_{\mathbf{e}_{j}}^{+}\right|,\left|\Psi_{\mathbf{e}_{j}}^{-}\right|\right)}{\max \left(\left|\Psi_{\mathbf{e}_{j}}^{+}\right|,\left|\Psi_{\mathbf{e}_{j}}^{-}\right|\right)}, \quad j=1,2
$$

where the factor is bounded by $f \in[0,1], f=0$ is complete disambiguation, and $\mathrm{f}=1$ is complete ambiguity. 
6. The $x$-axis is selected as the eigenvector with the lowest disambiguation factor $f$

$$
\mathbf{x}= \begin{cases}\mathbf{e}_{1} & \text { if } \mathrm{f}_{1}<\mathrm{f}_{2} \\ \mathbf{e}_{2} & \text { otherwise }\end{cases}
$$

and the $y$-axis is calculated as $\mathbf{y}=\mathbf{z} \times \mathbf{x}$ to create a right-handed system.

7. Occasionally, the axis disambiguation is not robust. If both disambiguation factors are similar in value, then two LRFs are created. Calculate the factor ratio as

$$
r_{f}=\frac{\min \left(f_{1}, f_{2}\right)}{\max \left(f_{1}, f_{2}\right)}
$$

If $r_{f}>t_{r_{f}}$, then create two LRFs, one with $\mathbf{e}_{1}$ as the $x$-axis and the other with $\mathbf{e}_{2}$ as the $x$-axis. This threshold is set as $t_{\mathrm{rf}_{\mathrm{f}}}=0.8$. Additionally, if the smaller disambiguation factor is greater than a threshold $t_{\mathrm{f}}$, then there is absolute ambiguity of the cluster orientation and four LRFs are generated - one for each sign of each eigenvector as the $x$-axis. This threshold is set as $t_{\mathrm{f}}=0.925$.

Through the process of defining a cluster's LRF, we may simultaneously define its scale. Three scales are attributed to each cluster, one for each LRF axis. Scale values assist in cluster description through the histogram, and to make the recognition framework more efficient.

\subsubsection{Comments on SOL-CVFH}

The SOL-CVFH descriptor removes the assumption of a segmented scene that both OUR-CVFH and SOUR-CVFH retain. Since the LRF is 
disambiguated only by the cluster, and not the entire object, it is more robust for object recognition in cluttered scenes. SOL-CVFH also maintains the benefits of SOUR-CVFH, such as minimizing the affects of sensor resolution, the affects of measurement noise, and quick histogram matching using cluster scales. 


\section{Chapter 6}

\section{Feature Histogram Performance Assessment}

\subsection{Individual Object Pose Estimation}

SOUR-CVFH and SOL-CVFH are compared to OUR-CVFH for pose estimation errors on five models from the Stanford scanning repository and and the asteroid Itokawa as shown in Fig. 6.1. The original models are downsampled and repaired (if necessary) using Meshlab [12]. A Monte Carlo experiment of 500 runs is performed for each model individually. Only the training database of a particular model is used to determine pose of that model. This experiment purely tests the descriptors' pose estimation abilities.

Training is performed on each model individually. Training ranges began at a distance equal to the maximum object dimension and finished at a distance twice the maximum object dimension, with eight equally spaced steps between. The object was placed in an polyhedron with 642 viewing orientations. For SOUR-CVFH, all of the threshold values used are equal to those listed in Table 5.11 except $t_{\mathbf{n}}=\cos \left(2^{\circ}\right)$ and $t_{J}=0.5$.

The pose estimation results for the 500 run Monte Carlo experiment are shown throughout Fig. 6.2, Fig. 6.3, Fig. 6.4, Fig. 6.5, and Fig. 6.6 for

each model individually. The combined statistics for each model are shown in 


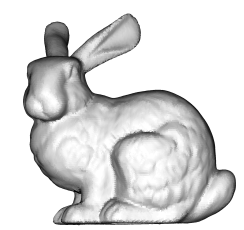

$m_{\mathbf{v}}=34834$

(a) $m_{f}=69451$

$\bar{e}=1.00$

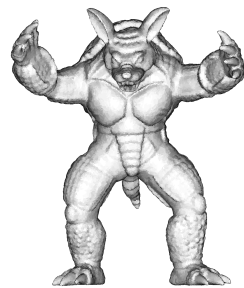

$$
m_{\mathbf{v}}=50002
$$

(b)

$$
\begin{gathered}
m_{f}=100000 \\
\bar{e}=0.95
\end{gathered}
$$

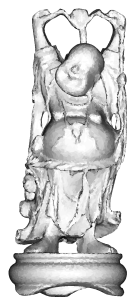

(c) $m_{f}=49971$

$\bar{e}=5.68$

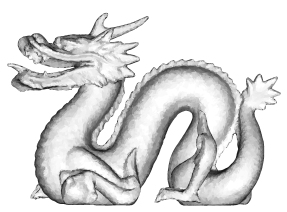

(d)

$$
\begin{aligned}
m_{\mathbf{v}} & =24982 \\
m_{f} & =49973 \\
\bar{e} & =4.65
\end{aligned}
$$

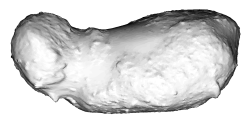

$m_{\mathbf{v}}=40002$

(e) $m_{f}=80000$

$\bar{e}=1.44$

Figure 6.1: (a)-(d) Models from the Standford scanning repository and (e) the asteroid Itokawa used for SOUR-CVFH testing. The number of vertices is $m_{\mathbf{v}}$, number of faces is $m_{f}$, and the average edge length is $\bar{e}$.

Table 6.1 with the best and worst performances highlighted in green and red respectively for each model and category. The number of histogram matches necessary are shown in Fig. 6.7 for all models.

According to Table 6.1, SOUR-CVFH performs comparable to or better than OUR-CVFH for each model; SOL-CVFH mostly performs in between. The largest improvement comes in the number of histogram matches necessary for each method to estimate pose as shown in Fig. 6.7. Because SOUR-CVFH and SOL-CVFH eliminate certain matches based on the cluster scales, they systematically reduce the number of histogram matches necessary by nearly two orders of magnitude. This is a large reduction in computational 
expense for SOUR-CVFH and SOL-CVFH .

The second through fourth rows of Fig. 6.2 through Fig. 6.6 show all pose estimation as a function of cost metric value for OUR-CVFH, SOURCVFH, and SOL-CVFH . Recall that the cost metric for OUR-CVFH is unbounded and only returns a pose estimate if it is positive. The cost metric for SOUR-CVFH and SOL-CVFH is bounded and returns a pose estimate in every instance. This conveniently permits the use of a cutoff threshold to eliminate SOUR-CVFH and SOL-CVFH pose estimates that return a cost metric below a certain threshold. For this experiment, we selected $t_{J}=0.5$ as shown by the vertical dotted line in the third and fourth rows. This threshold eliminates most of the poor SOUR-CVFH pose estimates. Despite also removing some good pose estimates with small cost metric values, SOUR-CVFH still returns more pose estimates than OUR-CVFH . Pose estimation performance could be improved with variable cost metric threshold depending on the model. 
Angle Error
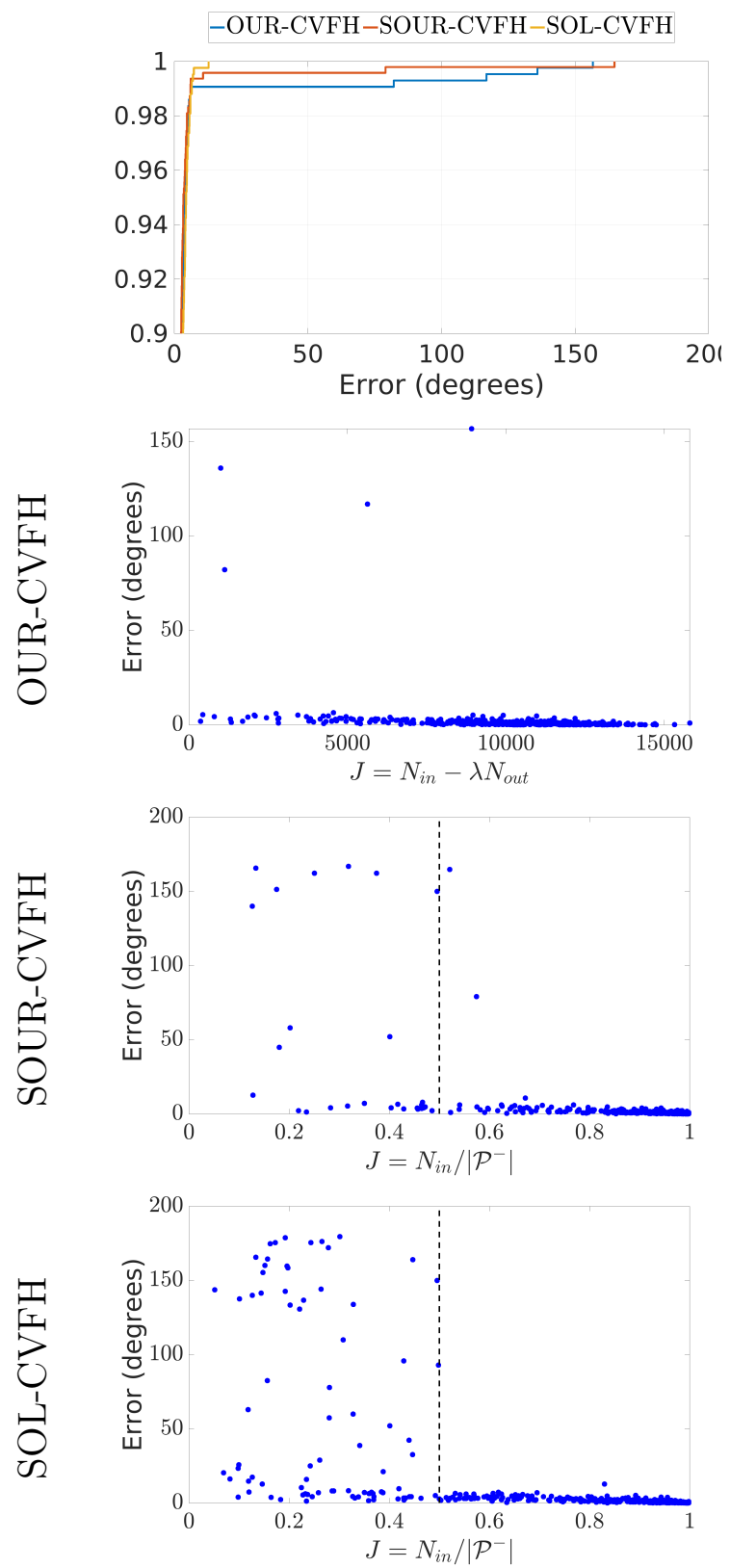

Range Error
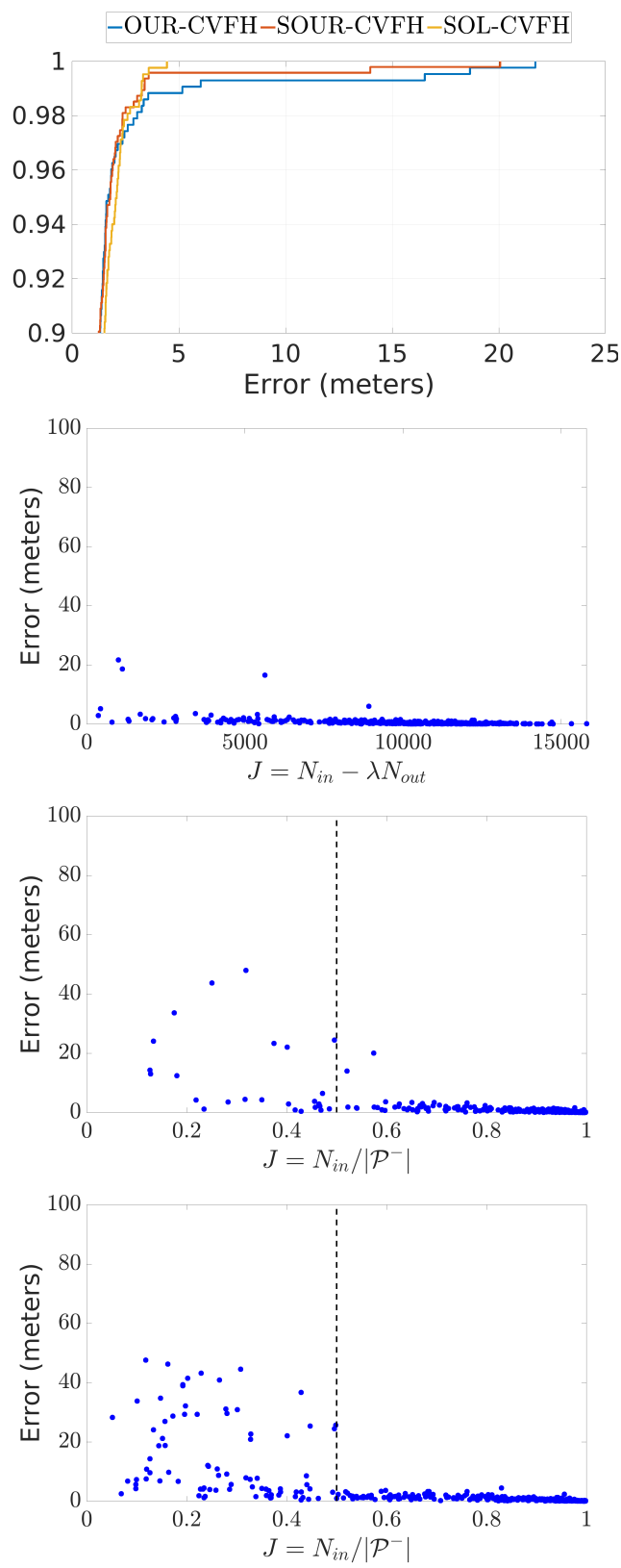

Figure 6.2: Pose estimation results of 500 run Monte Carlo comparing OURCVFH to SOUR-CVFH for the model Armadillo. 
Angle Error
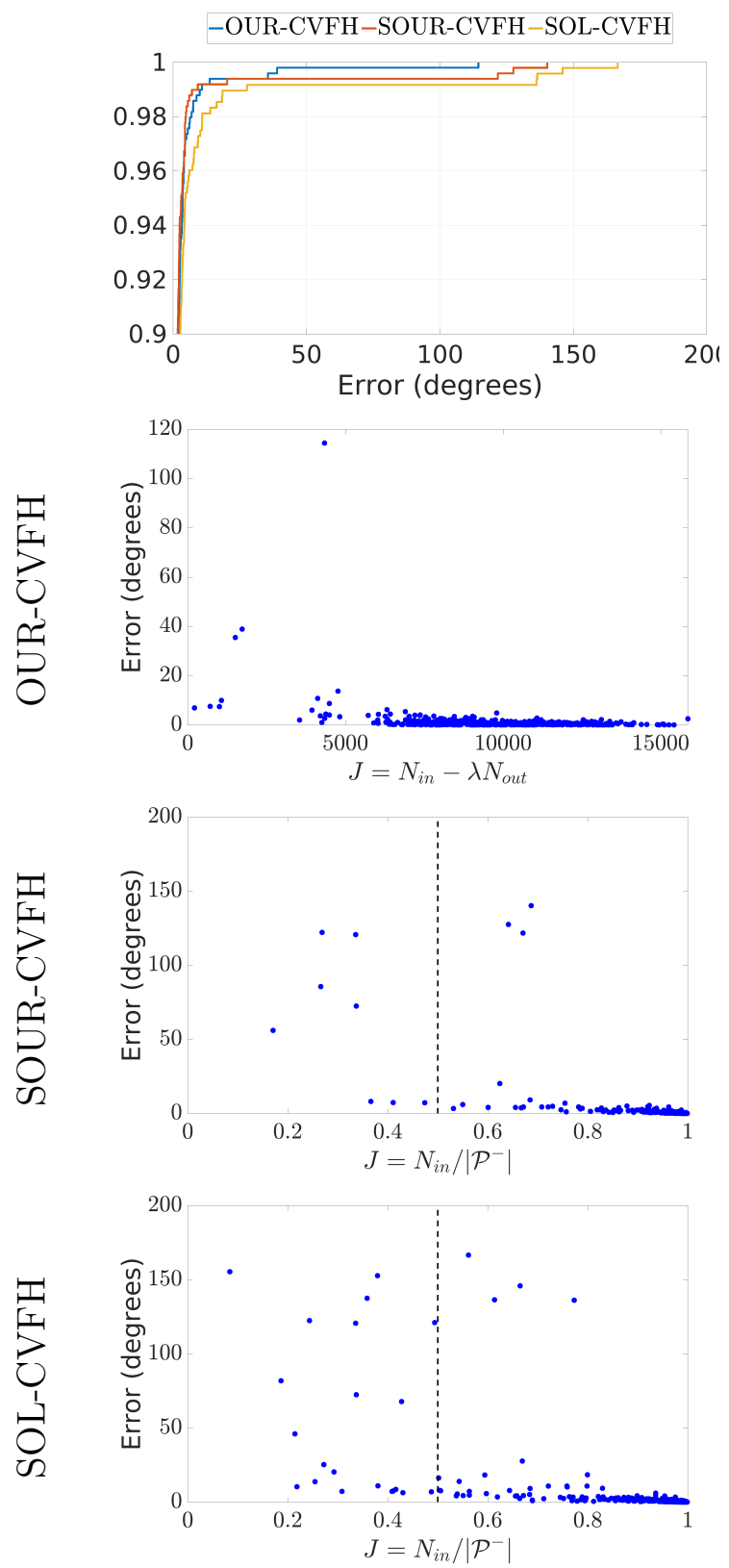

Range Error
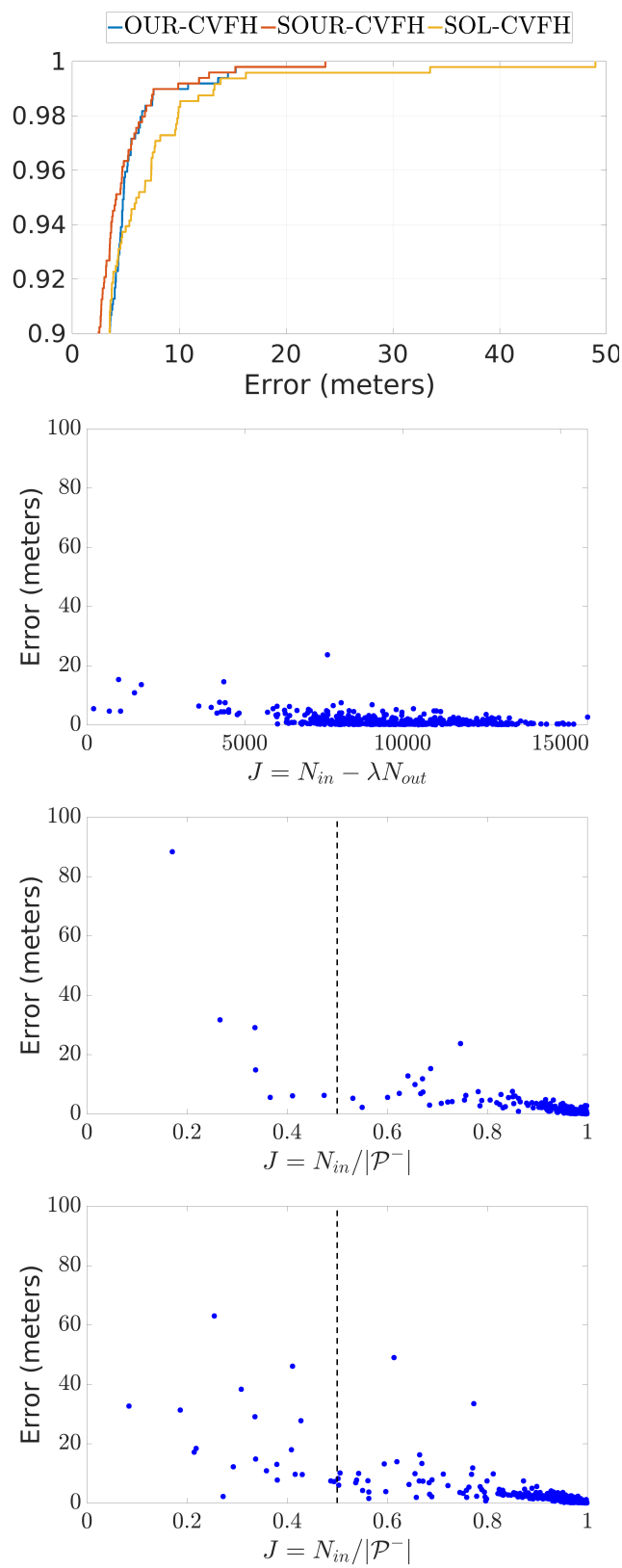

Figure 6.3: Pose estimation results of 500 run Monte Carlo comparing OURCVFH to SOUR-CVFH for the model Buddha. 
Angle Error
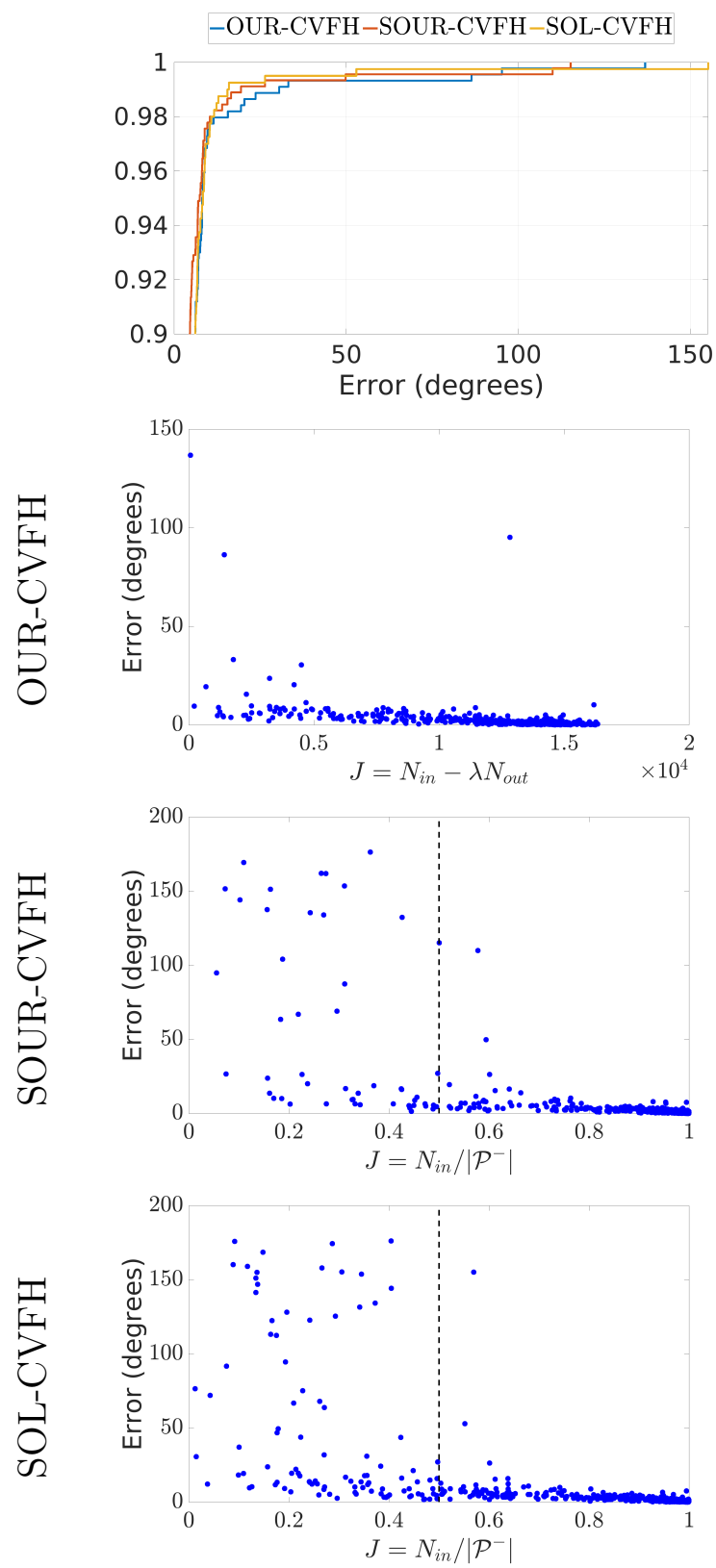

Range Error
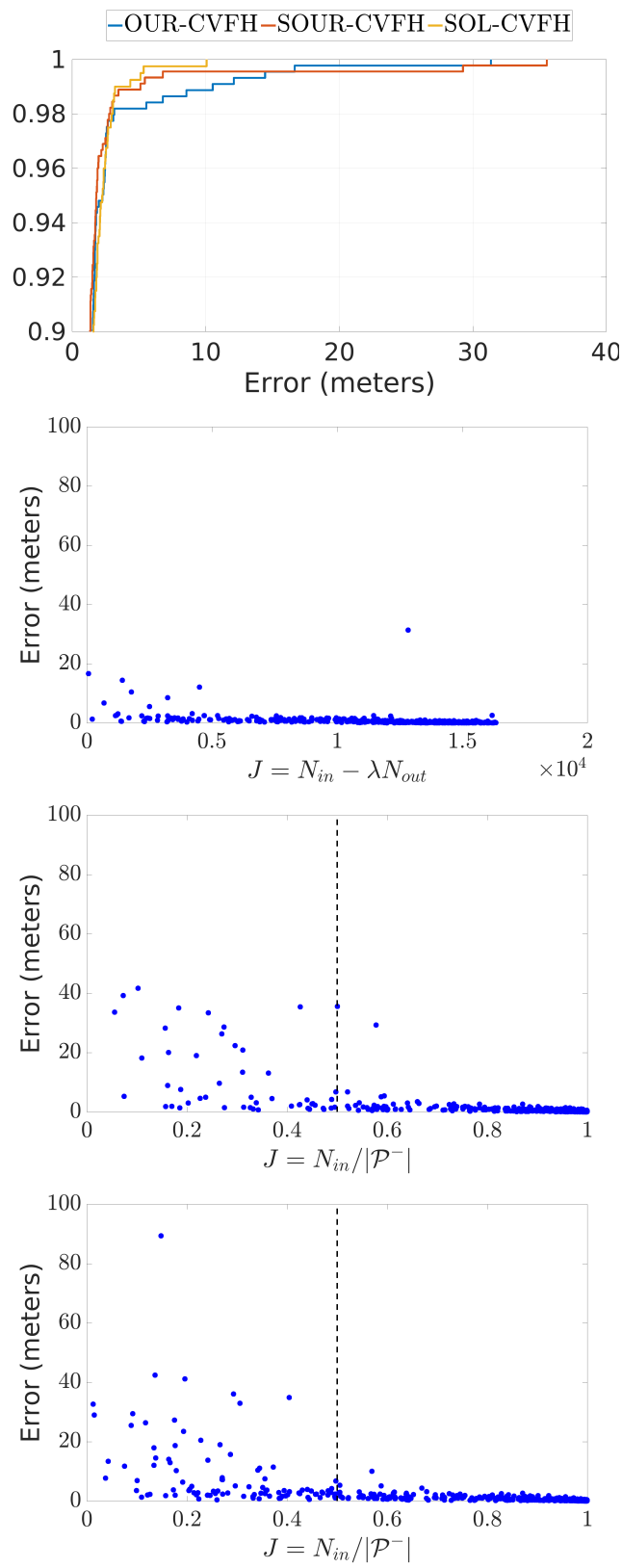

Figure 6.4: Pose estimation results of 500 run Monte Carlo comparing OURCVFH to SOUR-CVFH for the model Bunny. 
Angle Error
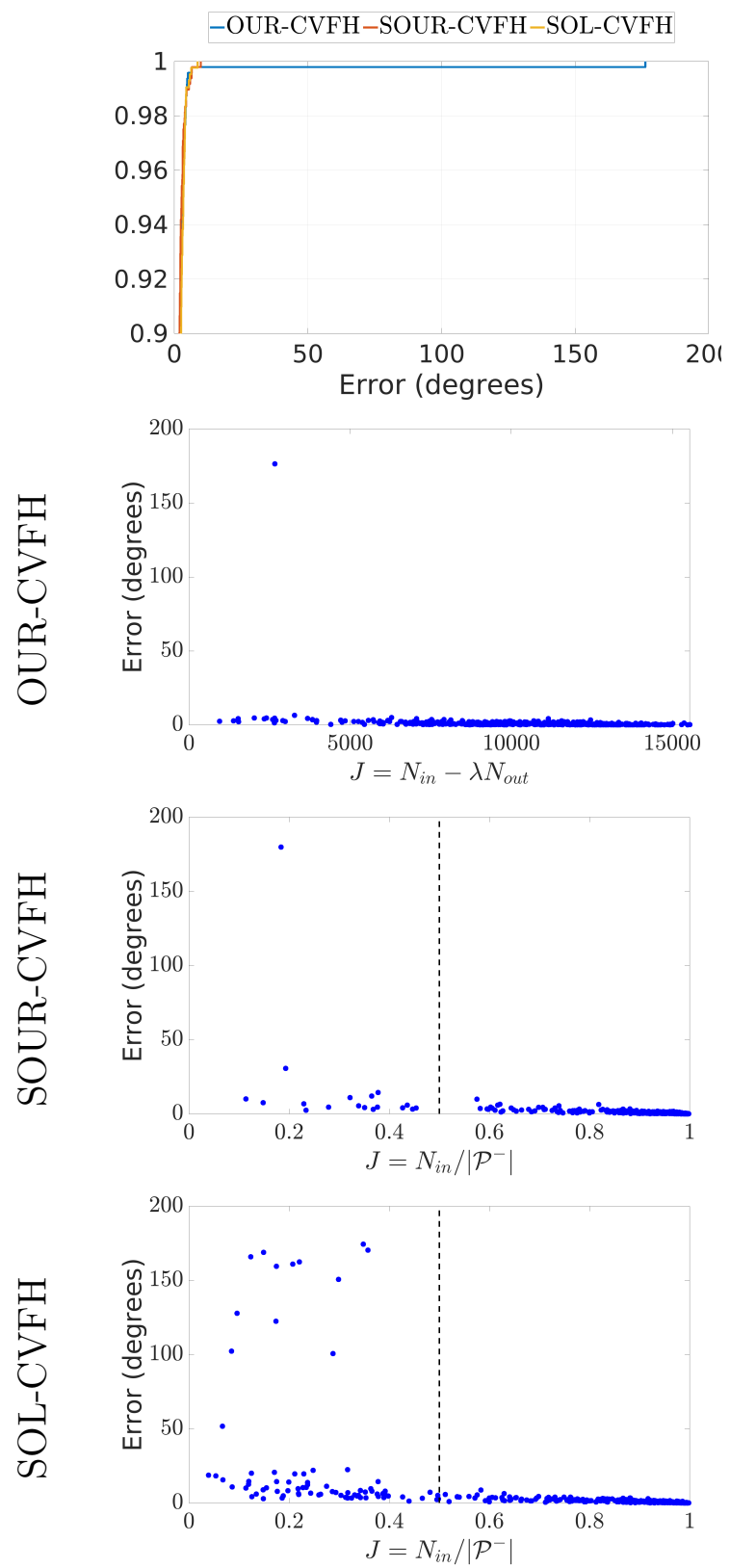

Range Error
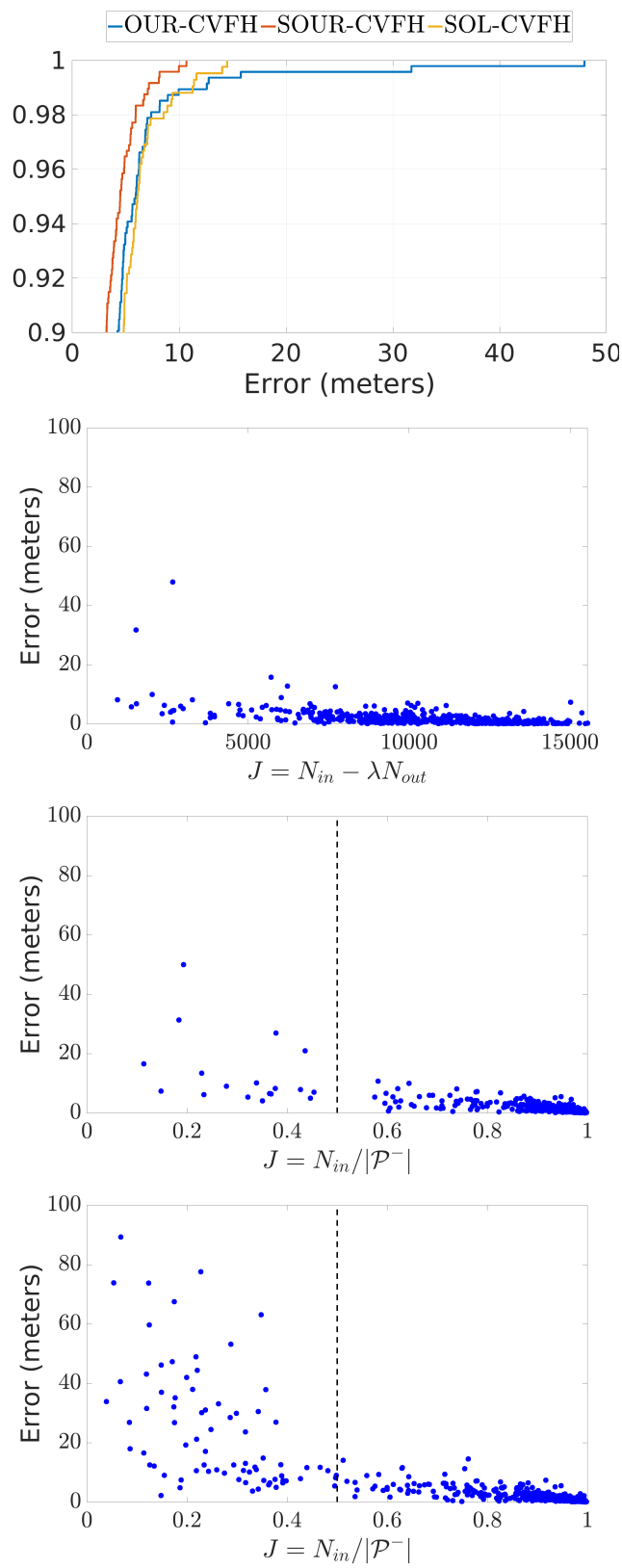

Figure 6.5: Pose estimation results of 500 run Monte Carlo comparing OURCVFH to SOUR-CVFH for the model Dragon. 
Angle Error
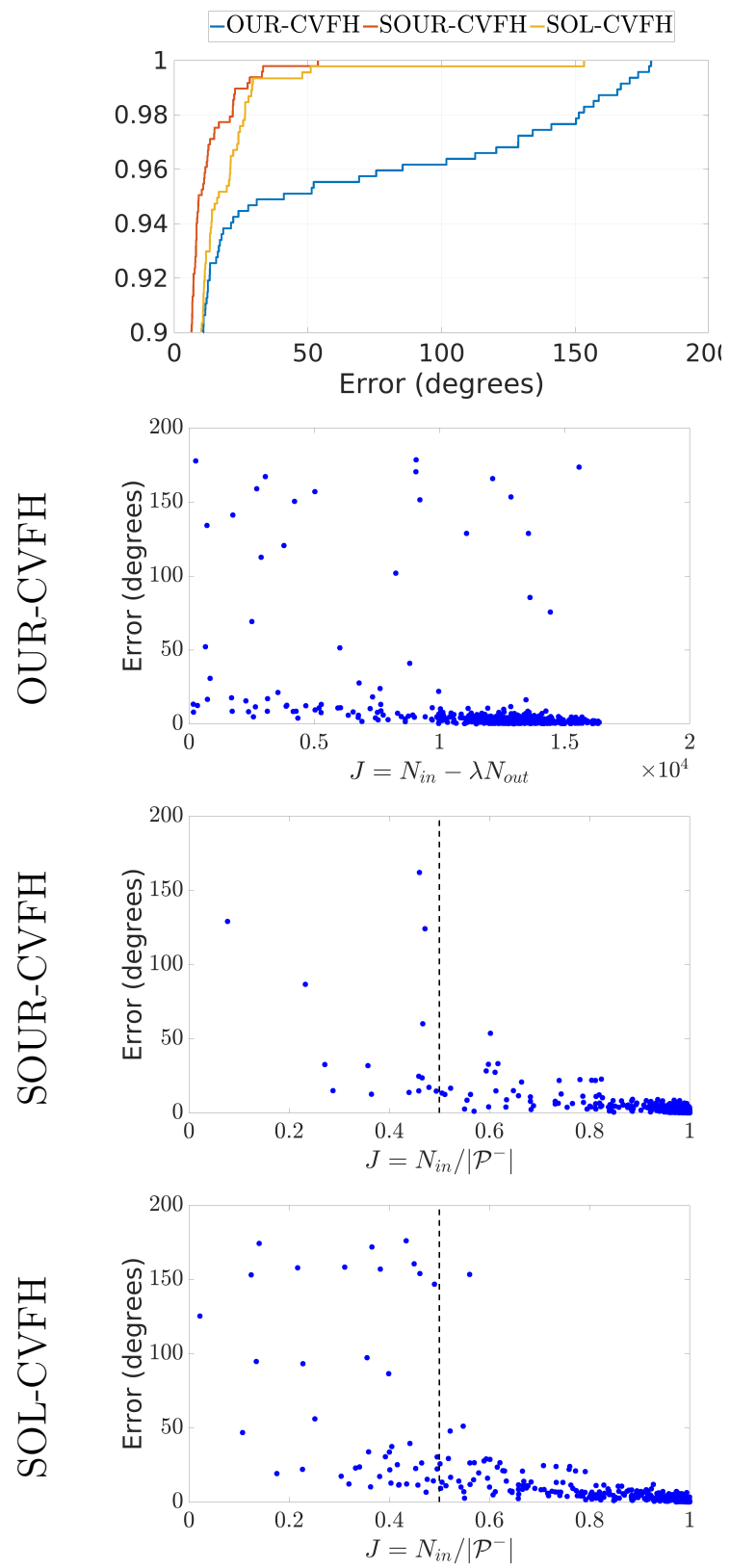

Range Error
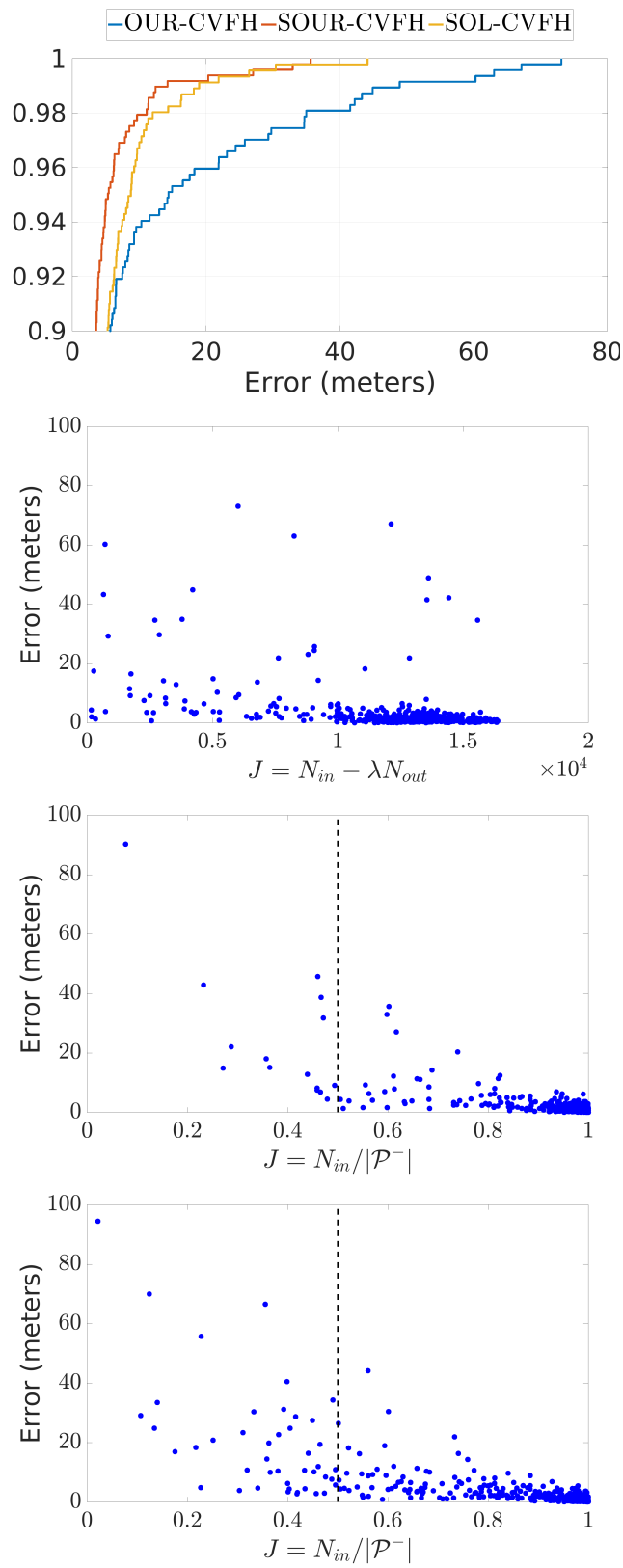

Figure 6.6: Pose estimation results of 500 run Monte Carlo comparing OURCVFH to SOUR-CVFH for the model Itokawa. 


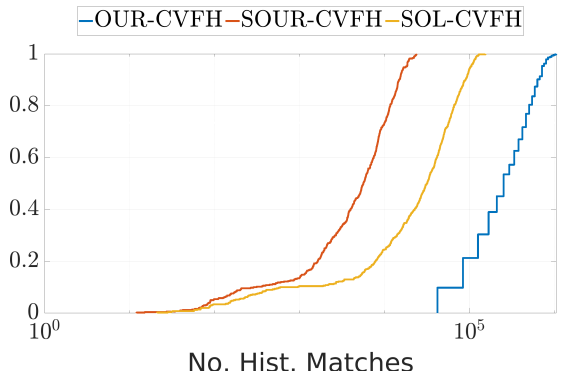

(a) armadillo

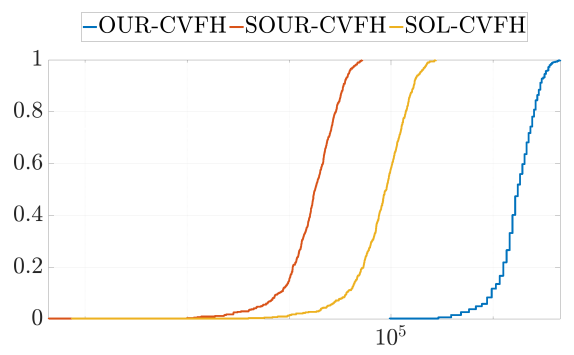

No. Hist. Matches

(c) bunny

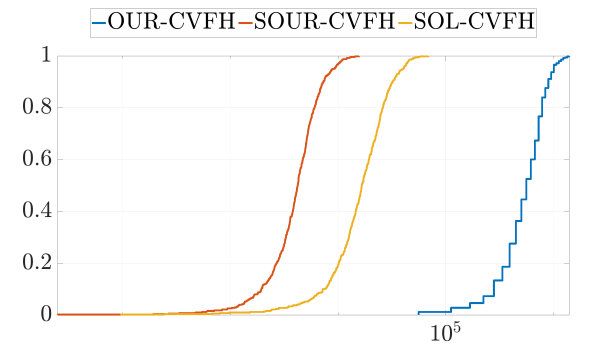

No. Hist. Matches

(b) buddha

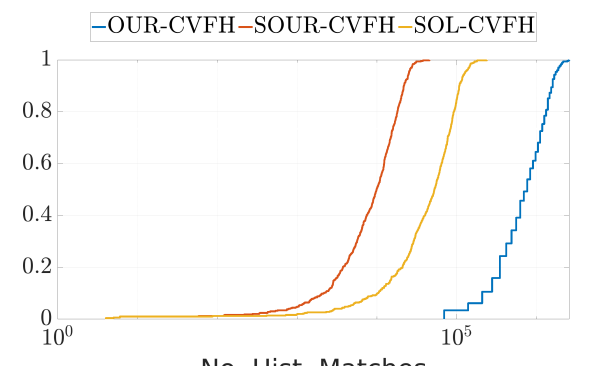

No. Hist. Matches

(d) dragon

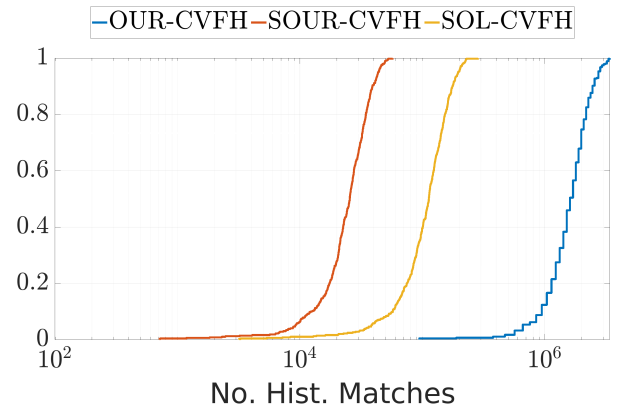

(e) itokawa

Figure 6.7: Number of histogram matches for all five models for OUR-CVFH, SOUR-CVFH, and SOL-CVFH . 
Table 6.1: Statistics for the 500 run Monte Carlo experiment comparing OUR-CVFH , SOUR-CVFH , and SOL-CVFH for five models. Settings of $t_{J}=0.5$.

\begin{tabular}{|c|c|c|c|c|c|c|}
\hline & \multicolumn{3}{|c|}{ Armadillo } & \multicolumn{3}{|c|}{ Buddha } \\
\hline & OUR-CVFH & SOUR-CVFH & SOL-CVFH & OUR-CVFH & SOUR-CVFH & SOL-CVFH \\
\hline \multicolumn{7}{|c|}{ Num. Pose Estimates } \\
\hline & 428 & 473 & 417 & 493 & 492 & 479 \\
\hline \multicolumn{7}{|l|}{ Angle Error } \\
\hline Mean (deg.) & $2.41^{\circ}$ & $1.46^{\circ}$ & $1.31^{\circ}$ & $1.29^{\circ}$ & $1.42^{\circ}$ & $2.31^{\circ}$ \\
\hline Median (deg.) & $0.98^{\circ}$ & $0.52^{\circ}$ & $0.74^{\circ}$ & $0.45^{\circ}$ & $0.22^{\circ}$ & $0.35^{\circ}$ \\
\hline Std. Dev. (deg.) & $12.07^{\circ}$ & $8.42^{\circ}$ & $1.53^{\circ}$ & $5.78^{\circ}$ & $10.18^{\circ}$ & $13.50^{\circ}$ \\
\hline \multicolumn{7}{|l|}{ Range Error } \\
\hline Mean & $0.70 \mathrm{~m}$ & $0.53 \mathrm{~m}$ & $0.60 \mathrm{~m}$ & $1.45 \mathrm{~m}$ & $1.07 \mathrm{~m}$ & $1.63 \mathrm{~m}$ \\
\hline Median & $0.38 \mathrm{~m}$ & $0.21 \mathrm{~m}$ & $0.32 \mathrm{~m}$ & $0.75 \mathrm{~m}$ & $0.47 \mathrm{~m}$ & $0.67 \mathrm{~m}$ \\
\hline Std. Dev. & $1.68 \mathrm{~m}$ & $1.24 \mathrm{~m}$ & $0.69 \mathrm{~m}$ & $2.06 \mathrm{~m}$ & $1.94 \mathrm{~m}$ & $3.39 \mathrm{~m}$ \\
\hline \multicolumn{7}{|c|}{ Num. Hist. Matches } \\
\hline Mean & $3.12 e^{5}$ & $6.76 e^{3}$ & $3.83 e^{4}$ & $5.81 e^{5}$ & $4.51 e^{3}$ & $1.86 e^{4}$ \\
\hline Median & $2.50 e^{5}$ & $5.63 e^{3}$ & $3.14 e^{4}$ & $5.58 e^{5}$ & $4.21 e^{3}$ & $1.65 e^{4}$ \\
\hline Std. Dev. & $2.25 e^{5}$ & $5.55 e^{3}$ & $3.26 e^{4}$ & $2.46 e^{5}$ & $2.29 e^{3}$ & $1.04 e^{4}$ \\
\hline
\end{tabular}


Table 6.1: Continued.

\begin{tabular}{|c|c|c|c|c|c|c|}
\hline & \multicolumn{3}{|c|}{ Bunny } & \multicolumn{3}{|c|}{ Dragon } \\
\hline & OUR-CVFH & SOUR-CVFH & SOL-CVFH & OUR-CVFH & SOUR-CVFH & SOL-CVFH \\
\hline \multicolumn{7}{|c|}{ Num. Pose Estimates } \\
\hline & 443 & 451 & 400 & 473 & 482 & 421 \\
\hline \multicolumn{7}{|l|}{ Angle Error } \\
\hline Mean (deg.) & $3.33^{\circ}$ & $2.59^{\circ}$ & $2.90^{\circ}$ & $1.38^{\circ}$ & $0.74^{\circ}$ & $0.96^{\circ}$ \\
\hline Median (deg.) & $1.68^{\circ}$ & $1.16^{\circ}$ & $1.47^{\circ}$ & $0.67^{\circ}$ & $0.35^{\circ}$ & $0.53^{\circ}$ \\
\hline Std. Dev. (deg.) & $9.33^{\circ}$ & $8.13^{\circ}$ & $8.54^{\circ}$ & $8.12^{\circ}$ & $1.05^{\circ}$ & $1.13^{\circ}$ \\
\hline \multicolumn{7}{|l|}{ Range Error } \\
\hline Mean & $0.83 \mathrm{~m}$ & $0.69 \mathrm{~m}$ & $0.68 \mathrm{~m}$ & $1.95 \mathrm{~m}$ & $1.27 \mathrm{~m}$ & $1.78 \mathrm{~m}$ \\
\hline Median & $0.44 \mathrm{~m}$ & $0.32 \mathrm{~m}$ & $0.39 \mathrm{~m}$ & $1.16 \mathrm{~m}$ & $0.68 \mathrm{~m}$ & $0.81 \mathrm{~m}$ \\
\hline Std. Dev. & $2.06 \mathrm{~m}$ & $2.24 \mathrm{~m}$ & $0.89 \mathrm{~m}$ & $3.18 \mathrm{~m}$ & $1.54 \mathrm{~m}$ & $2.20 \mathrm{~m}$ \\
\hline \multicolumn{7}{|c|}{ Num. Hist. Matches } \\
\hline Mean & $1.89 e^{6}$ & $1.99 e^{4}$ & $9.67 e^{4}$ & $8.33 e^{5}$ & $1.11 e^{4}$ & $5.73 e^{4}$ \\
\hline Median & $1.75 e^{6}$ & $1.80 e^{4}$ & $9.00 e^{4}$ & $7.63 e^{5}$ & $9.89 e^{3}$ & $5.25 e^{4}$ \\
\hline Std. Dev. & $7.69 e^{5}$ & $1.03 e^{4}$ & $5.12 e^{4}$ & $5.19 e^{5}$ & $7.74 e^{3}$ & $3.96 e^{4}$ \\
\hline
\end{tabular}


Table 6.1: Continued.

\begin{tabular}{|c|c|c|c|}
\hline & \multicolumn{3}{|c|}{ Itokawa } \\
\hline & OUR-CVFH & \multirow[t]{2}{*}{ SOUR-CVFH } & SOL-CVFH \\
\hline \multicolumn{3}{|c|}{ Num. Pose Estimates } & \\
\hline & 470 & 485 & 456 \\
\hline \multicolumn{4}{|l|}{ Angle Error } \\
\hline Mean (deg.) & $9.78^{\circ}-x-1$ & $2.94^{\circ}$ & $4.54^{\circ}$ \\
\hline Median (deg.) & $2.44^{\circ}$ & $1.51^{\circ}$ & $2.05^{\circ}$ \\
\hline Std. Dev. (deg.) & $29.12^{\circ}$ & $4.82^{\circ}$ & $9.27^{\circ}$ \\
\hline \multicolumn{4}{|l|}{ Range Error } \\
\hline Mean (m.) & $3.59 \mathrm{~m}$ & $1.75 \mathrm{~m}$ & $2.38 \mathrm{~m}$ \\
\hline Median (m.) & $1.43 \mathrm{~m}$ & $0.93 \mathrm{~m}$ & $1.22 \mathrm{~m}$ \\
\hline Std. Dev. (m.) & $8.52 \mathrm{~m}$ & $3.17 \mathrm{~m}$ & $3.85 \mathrm{~m}$ \\
\hline \multicolumn{4}{|c|}{ Num. Hist. Matches } \\
\hline Mean & $1.67 e^{6}$ & $2.58 e^{4}$ & $1.14 e^{5}$ \\
\hline Median & $1.60 e^{6}$ & $2.55 e^{4}$ & $1.13 e^{5}$ \\
\hline Std. Dev. & $6.10 e^{5}$ & $1.03 e^{4}$ & $4.67 e^{4}$ \\
\hline
\end{tabular}

\subsection{Object Recognition in Cluttered Scenes}

SOUR-CVFH and SOL-CVFH are compared to OUR-CVFH for object recognition in cluttered scenes. We use the SHOT Dataset $3[2]$ which is constructed using the spacetime stereo technique $[8,9]$. It contains four sets; three sets contain four scenes each, and the fourth set contains three scenes. Each scene in a set has increasing amounts of clutter blocking the model of interest. A select sampling of the scenes is shown in Fig. 6.10 and overlaid with clusters in Fig. 6.11. All four sets have a single measurement of two different

models which are shown in Fig. 6.8 and overlaid with clusters in Fig. 6.9. The 
experiment objective is to (1) perform object recognition and (2) to estimate pose.

Knowledge that the scenes are cluttered such that portions of the models may be occluded informs the thresholds settings in Table 5.11. First, large clusters are not expected to be visible in the scene due to the clutter. Small clusters are more likely to be completely visible, so we set $t_{\bar{n}}=\cos \left(3^{\circ}\right)$ which prioritizes smaller clusters. Second, the clutter may occlude one of the cluster axes such that the scale matching procedure is too restrictive so we only require one axis to meet the scale ration threshold (instead of both axes). Third, a cluttered scene results in less point-cloud overlap than when matching a single object causing the cost metric value to be much lower than the previous experiment. Therefore, we set $t_{J}=0.1$ and the remaining parameter values are set in Table 5.11.

Pose estimation results for each set of scenes is shown in Fig. 6.12 using CDF plots. All pose errors as a function of cost metric are shown in Fig. 6.13 with a vertical black dotted line corresponding to the cost metric threshold of $t_{J}=0.1$. The number of histogram matches performed for each descriptor is shown in Fig. 6.14 using CDF plots. Finally, the statistics for these experiments are shown in Table 6.2 with the best and worst performances highlighted in green and red respectively for each set. It is possible to correctly identify an object but return have the pose estimate rejected by the cost metric threshold, so we list this data separately.

Recall that both OUR-CVFH and SOUR-CVFH carry the assumption 


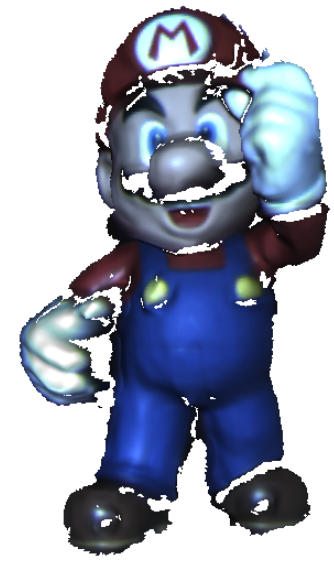

(a) Set 1,4

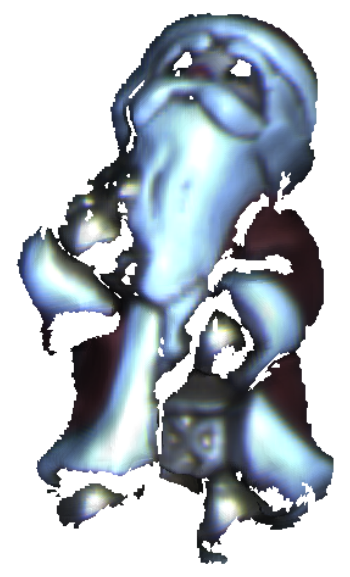

(d) Set 2

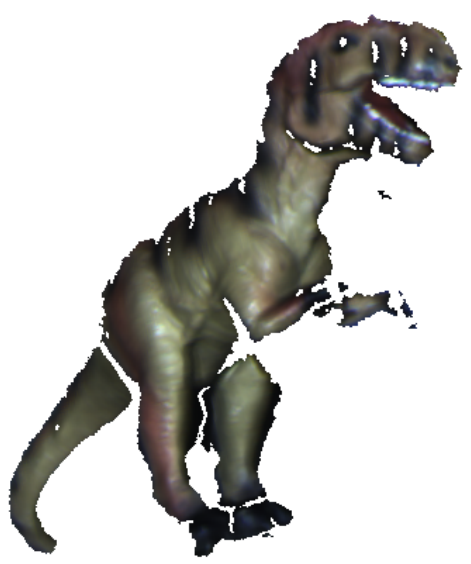

(b) Set 1

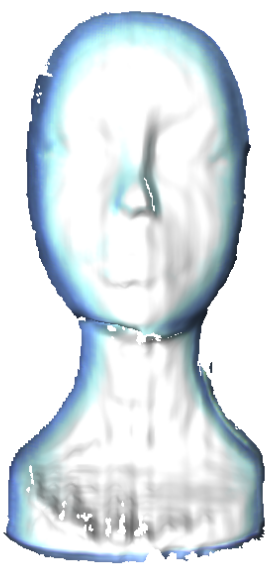

(e) Set 3

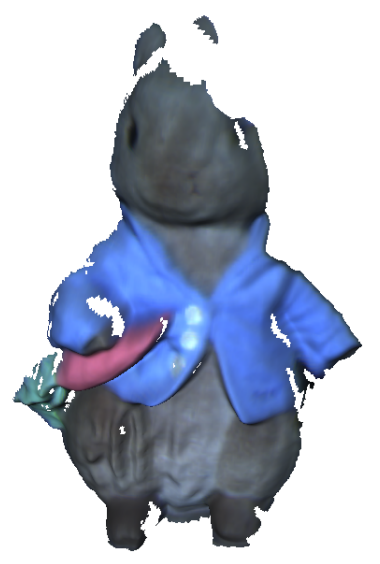

(c) Set 2, 4

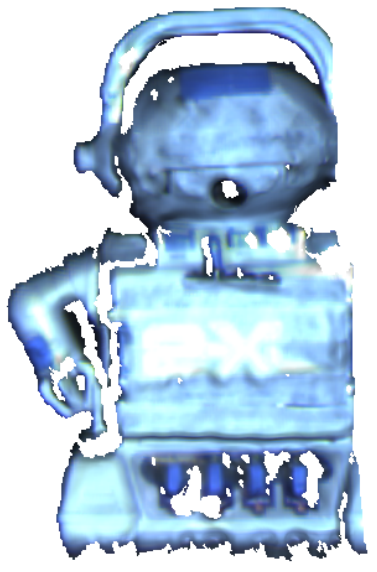

(f) Set 3

Figure 6.8: Models of the SHOT Dataset 3 [2] used for object recognition in cluttered scenes. Each model is found in at least one set. 

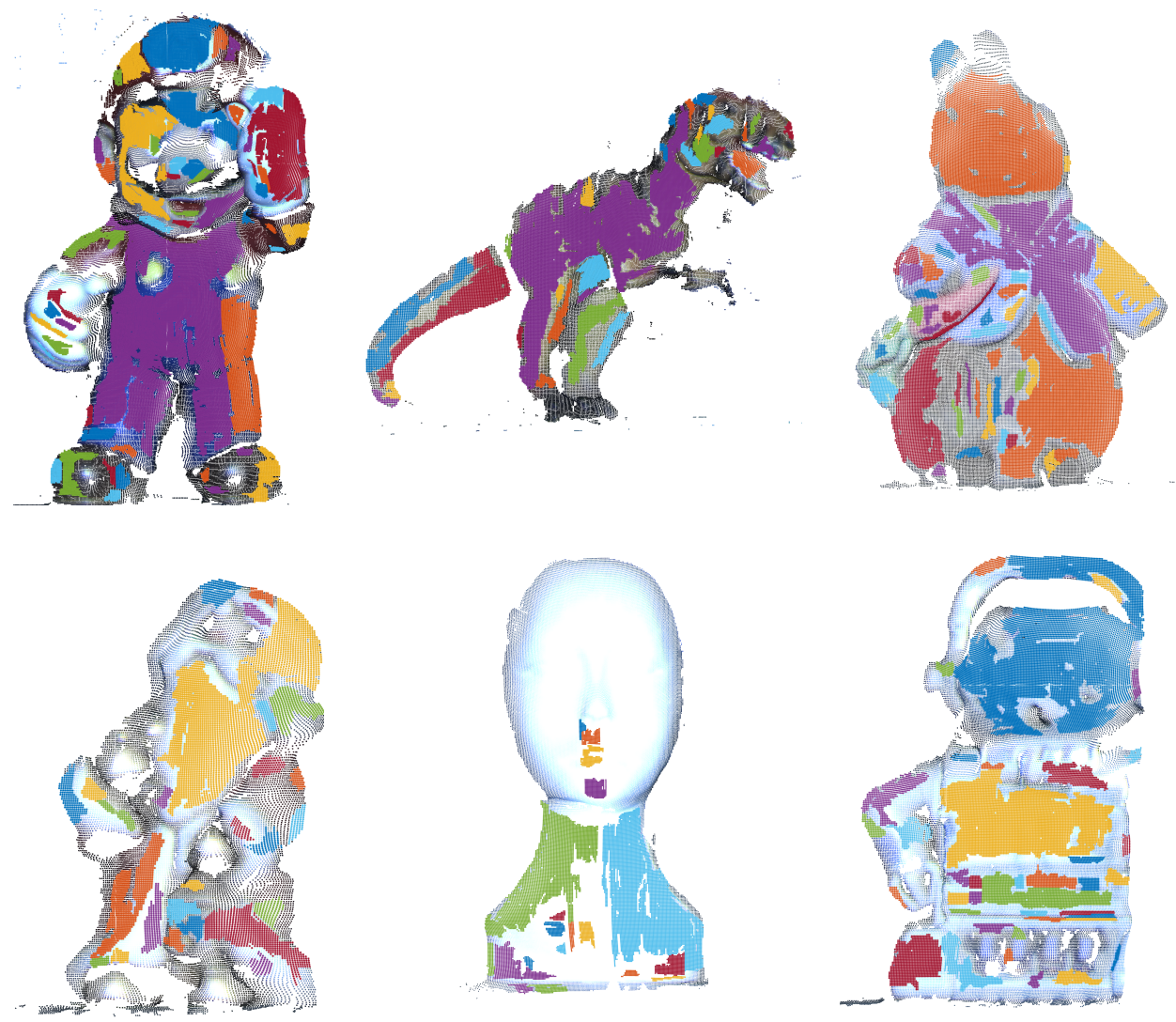

Figure 6.9: SOUR-CVFH clusters overlaid on models of the SHOT Dataset 3. 


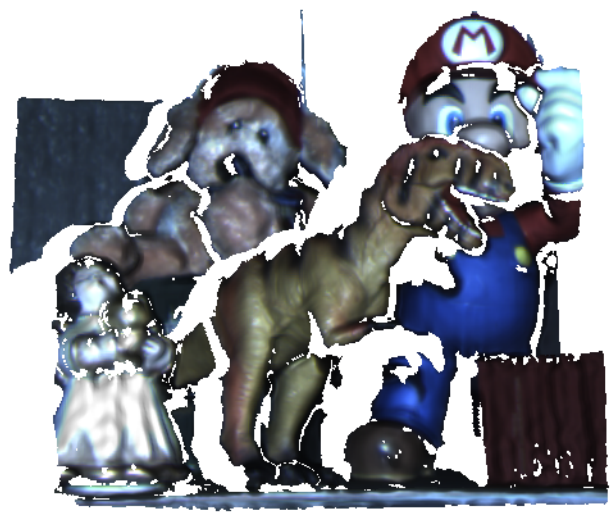

(1) Set 1 , Scene 1

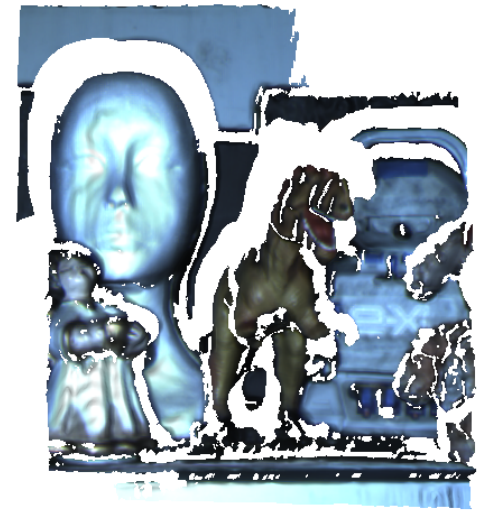

(c) Set 3, Scene 1

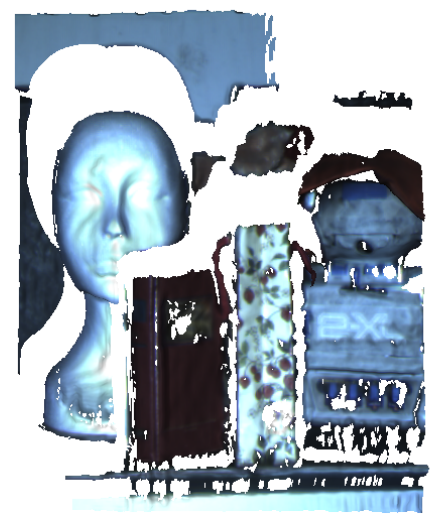

(e) Set 3, Scene 4

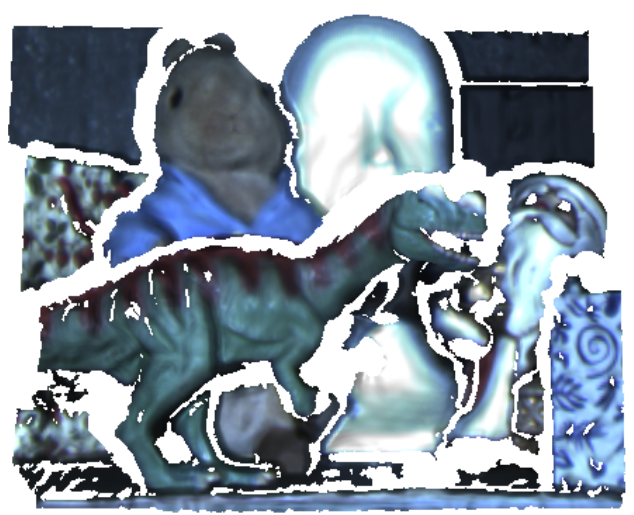

(b) Set 2, Scene 1

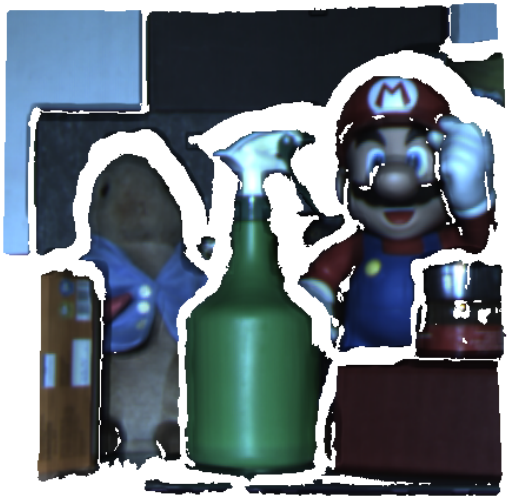

(d) Set 4, Scene 1

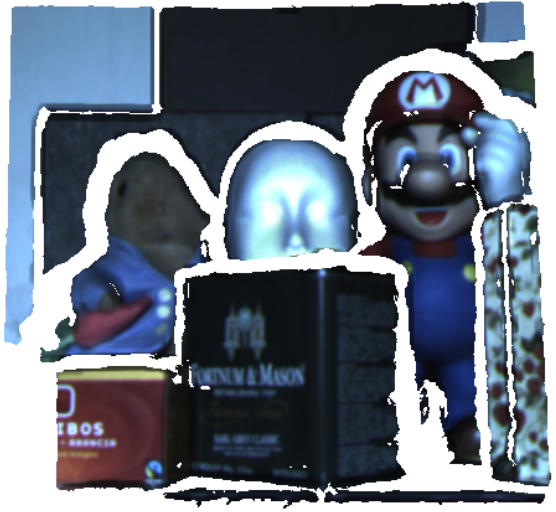

(f) Set 4 , Scene 3

Figure 6.10: Selected scenes from the SHOT Dataset 3 [2] used for object recognition in cluttered scenes. 

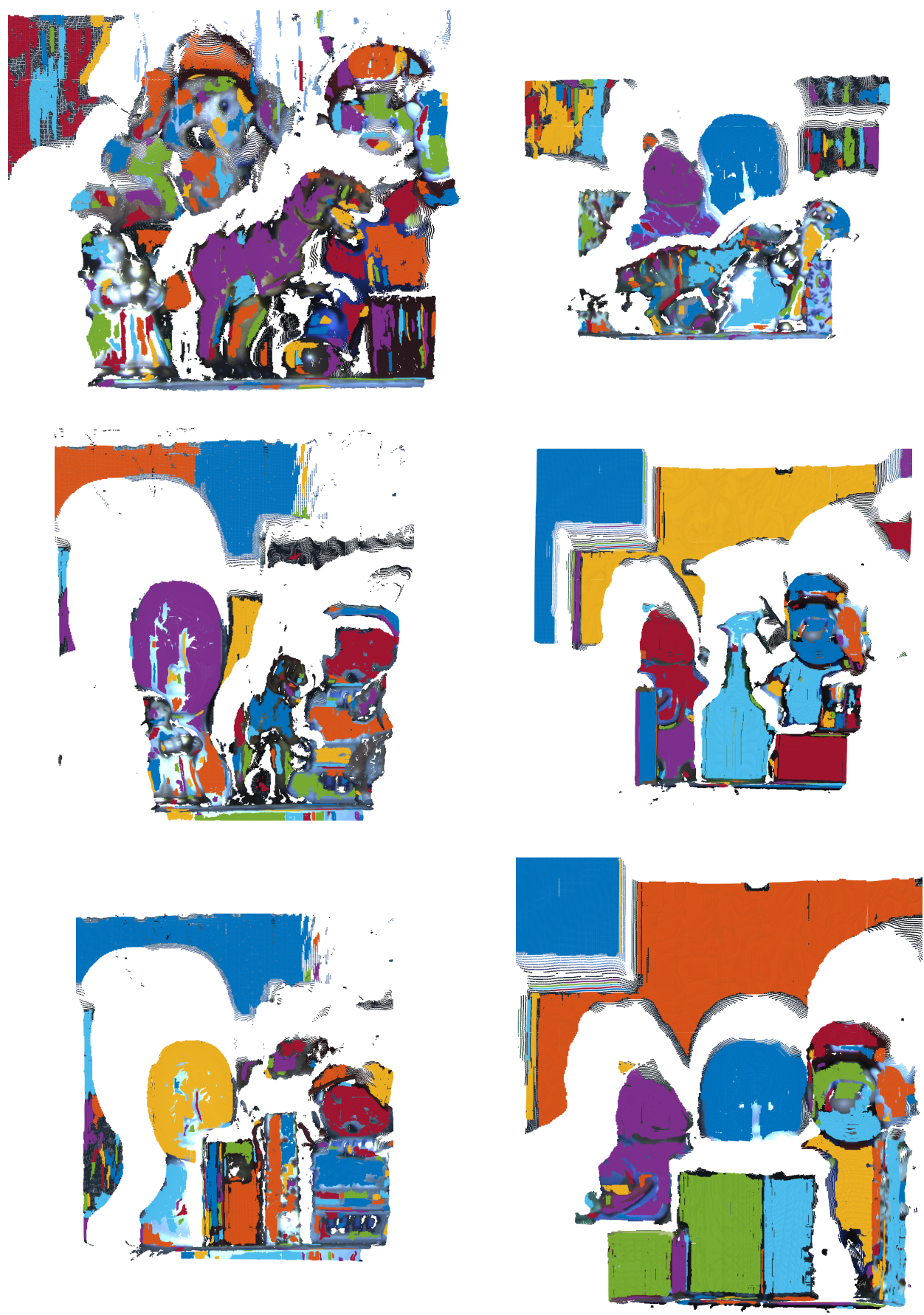

Figure 6.11: SOUR-CVFH clusters overlaid on selected scenes from the SHOT Dataset 3 . 
of a segmented scene and that the SGURF is disambiguated by the entire measured object. We did not apply any method of scene segmentation in this experiment, thus the assumption is not satisfied. As a result, OURCVFH only provided one pose estimate on Set 2 Scene 3 for which the model of interest was in front and completely unobstructed. Despite breaking the assumption, SOUR-CVFH still correctly identifies the object more than half of the time. SOL-CVFH does not carry the segmented scene assumption, and thus returns the most pose estimates and the most correctly identified objects. Interestingly, both SOUR-CVFH and SOL-CVFH perform similarly on all sets and scenes despite the SOUR-CVFH assumption of a segmented scene. This is a results of the scale matching procedure. 

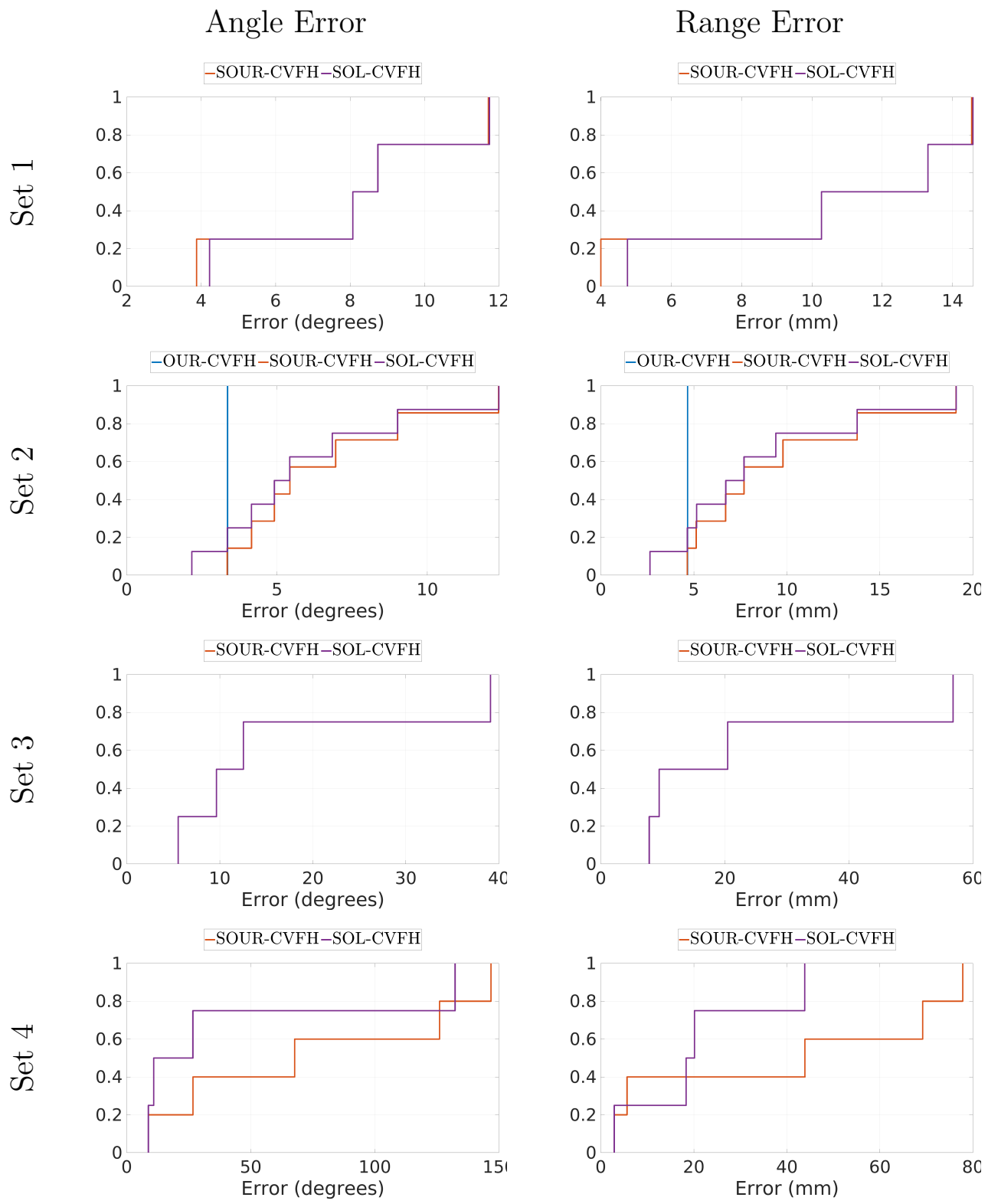

Figure 6.12: Angle and range errors in CDF plots for each descriptor separated by set for the SHOT Dataset 3. OUR-CVFH shown in blue, SOURCVFH shown in orange, and SOL-CVFH shown in purple. 

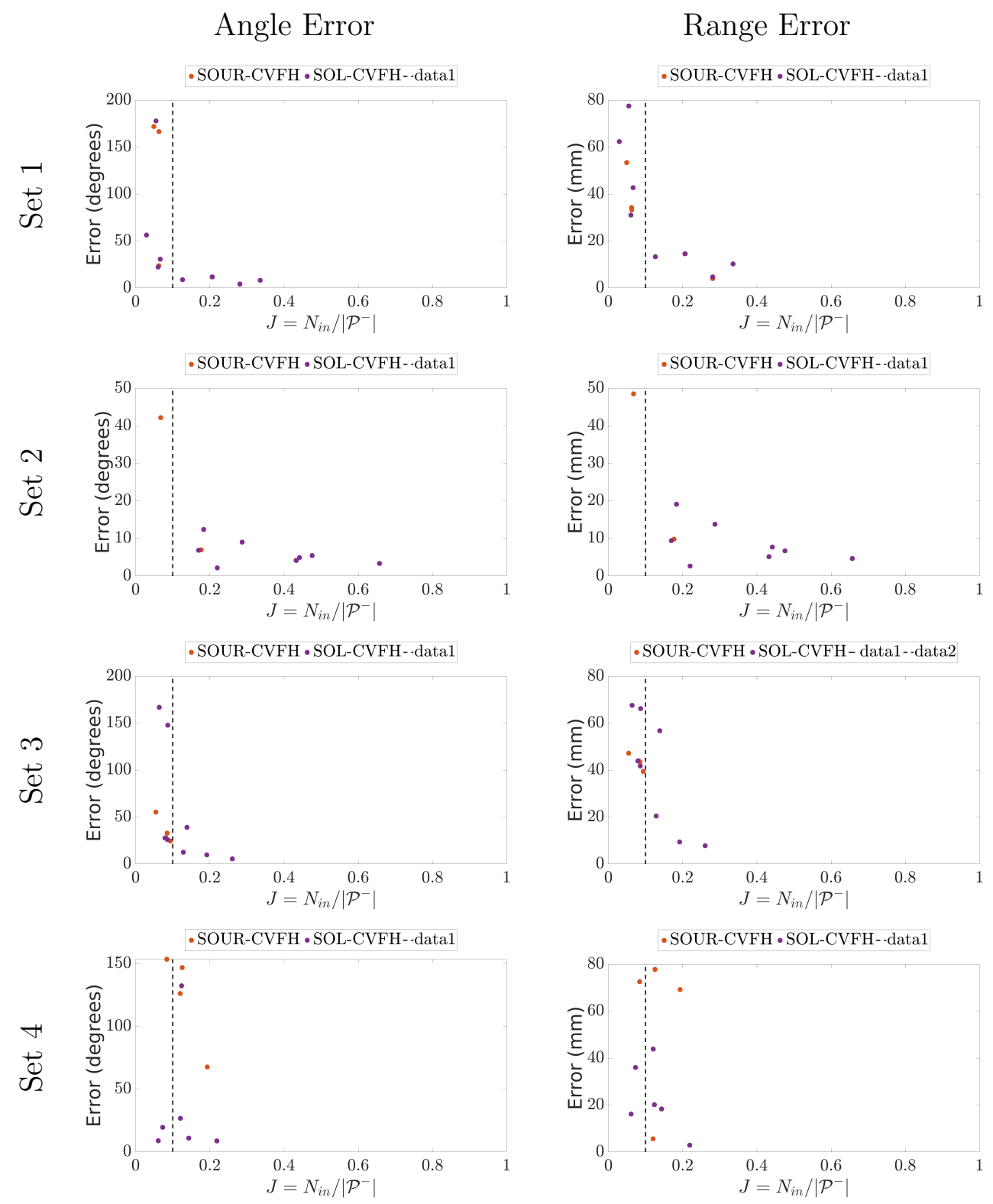

Figure 6.13: All angle and range errors as a function of cost metric for each descriptor separated by set for the SHOT Dataset 3. SOUR-CVFH shown in orange and SOL-CVFH shown in purple. OUR-CVFH not shown because the cost metric is unnormalized and only succeeded in one instance. 


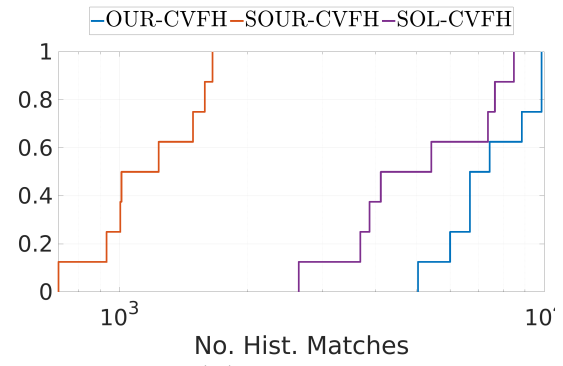

(a) Set 1

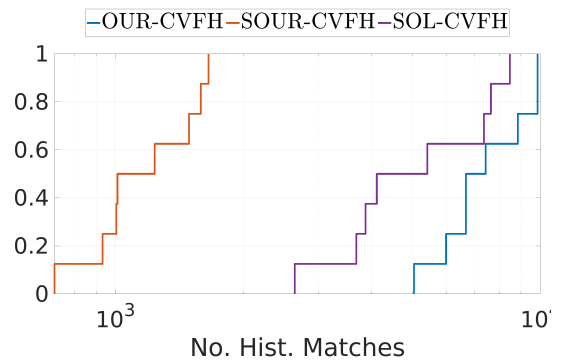

(a) Set 3

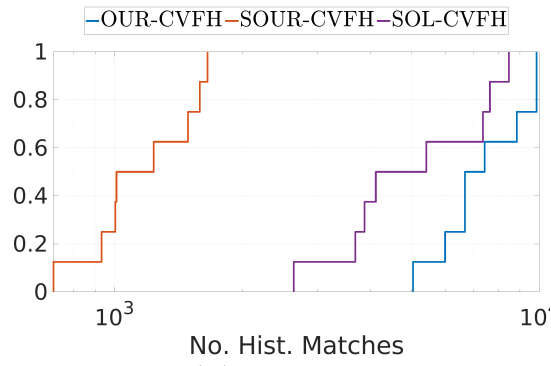

(a) Set 2

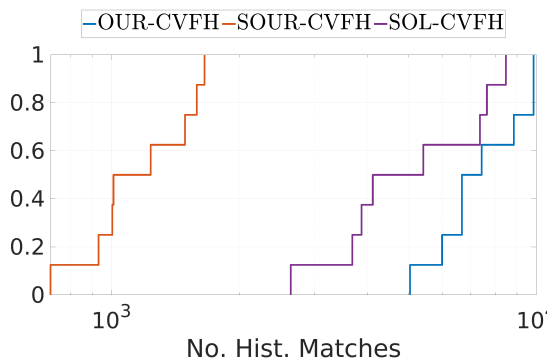

(a) Set 4

Figure 6.14: Number of histogram matches for each descriptor separated by set for the SHOT Dataset 3. OUR-CVFH shown in blue, SOUR-CVFH shown in orange, and SOL-CVFH shown in purple. 
Table 6.2: Statistics for the SHOT Dataset 3 experiment comparing OUR-CVFH, SOUR-CVFH , and SOL-CVFH for object recognition in cluttered scenes. Settings of $t_{\mathbf{n}}=\cos \left(3^{\circ}\right), t_{J}=0.1$, and only requiring one axis to meet the scale ratio threshold of $t_{\sigma}=0.9$. Numbers in parenthesis indicate the total amount possible.

\begin{tabular}{|c|c|c|c|c|c|c|}
\hline & \multicolumn{3}{|c|}{ Set 1} & \multicolumn{3}{|c|}{ Set 2} \\
\hline & OUR-CVFH & SOUR-CVFH & SOL-CVFH & OUR-CVFH & SOUR-CVFH & SOL-CVFH \\
\hline \multicolumn{7}{|c|}{ No. Pose Estimates } \\
\hline & $0(8)$ & $4(8)$ & $4(8)$ & $1(8)$ & $7(8)$ & $8(8)$ \\
\hline \multicolumn{7}{|l|}{ No. Correct } \\
\hline & $0(8)$ & $6(8)$ & $6(8)$ & $1(8)$ & $8(8)$ & $8(8)$ \\
\hline \multicolumn{7}{|l|}{ Angle Error } \\
\hline Mean (deg.) & - & $8.11^{\circ}$ & $8.20^{\circ}$ & $3.35^{\circ}$ & $6.59^{\circ}$ & $6.03^{\circ}$ \\
\hline Median (deg.) & - & $8.41^{\circ}$ & $8.41^{\circ}$ & $3.35^{\circ}$ & $5.43^{\circ}$ & $5.17^{\circ}$ \\
\hline Std. Dev. (deg.) & - & $3.23^{\circ}$ & $3.09^{\circ}$ & $3.35^{\circ}$ & $3.16^{\circ}$ & $3.32^{\circ}$ \\
\hline \multicolumn{7}{|l|}{ Range Error } \\
\hline Mean (mm) & - & 10.53 & 10.73 & 4.66 & 9.55 & 8.64 \\
\hline Median (mm) & - & 11.78 & 11.79 & 4.66 & 7.69 & 7.21 \\
\hline Std. Dev. (mm) & - & 4.71 & 4.38 & 4.66 & 5.23 & 5.41 \\
\hline \multicolumn{7}{|l|}{ No. Hist. Matches } \\
\hline Mean & 7549 & 1204 & 5405 & 5893 & 1015 & 4273 \\
\hline Median & 7057 & 1123 & 4770 & 5815 & 1010 & 4072 \\
\hline Std. Dev. & 1794 & 342 & 2168 & 1719 & 284 & 1405 \\
\hline
\end{tabular}


Table 6.3: Continued.

\begin{tabular}{|c|c|c|c|c|c|c|}
\hline & \multicolumn{3}{|c|}{ Set 3} & \multicolumn{3}{|c|}{ Set 4} \\
\hline & OUR-CVFH & SOUR-CVFH & SOL-CVFH & OUR-CVFH & SOUR-CVFH & SOL-CVFH \\
\hline \multicolumn{7}{|c|}{ No. Pose Estimates } \\
\hline & $0(8)$ & $4(8)$ & $4(8)$ & $0(6)$ & $5(6)$ & $4(6)$ \\
\hline \multicolumn{7}{|l|}{ No. Correct } \\
\hline & $0(8)$ & $7(8)$ & $6(8)$ & $0(6)$ & $2(6)$ & $5(6)$ \\
\hline $\begin{array}{l}\text { Angle Error } \\
\text { Mean (deg.) } \\
\text { Median (deg.) } \\
\text { Std. Dev. (deg.) }\end{array}$ & - & $\begin{array}{l}16.70^{\circ} \\
11.09^{\circ} \\
15.21^{\circ}\end{array}$ & $\begin{array}{l}16.70^{\circ} \\
11.09^{\circ} \\
15.21^{\circ}\end{array}$ & - & $\begin{array}{l}75.21^{\circ} \\
67.68^{\circ} \\
90.32^{\circ}\end{array}$ & $\begin{array}{l}44.64^{\circ} \\
18.76^{\circ} \\
59.01^{\circ}\end{array}$ \\
\hline \multicolumn{7}{|l|}{ Range Error } \\
\hline Mean (mm) & - & 23.61 & 23.61 & - & 39.88 & 21.30 \\
\hline Median (mm) & - & 14.93 & 14.93 & - & 43.88 & 19.23 \\
\hline Std. Dev. (mm) & - & 22.83 & 22.83 & - & 34.84 & 16.90 \\
\hline \multicolumn{7}{|l|}{ No. Hist. Matches } \\
\hline Mean & 4118 & 627 & 2926 & 2572 & 328 & 1533 \\
\hline Median & 3929 & 597 & 2848 & 2499 & 322 & 1526 \\
\hline Std. Dev. & 1578 & 241 & 1389 & 994 & 120 & 506 \\
\hline
\end{tabular}


The most significant improvement comes from the number of histogram matches necessary to estimate pose. OUR-CVFH routinely uses thousands of matches without returning any estimates. SOUR-CVFH and SOL-CVFH test scale similarity before matching histograms, thus speeding up the matching procedure. SOL-CVFH requires more histogram matches than SOURCVFH because its LRF is more ambiguous than the SOUR-CVFH SGURF. More ambiguity in the LRF yields more histograms per cluster and thus more histgogram matches. Number of histogram matches for SOUR-CVFH and SOL-CVFH are always less than OUR-CVFH .

Recall that the matching procedure maximizes point-cloud overlap with Eq. 5.57. Also recall that the best $m_{d}$ histogram matches are further processed using ICP. If one of these $m_{d}$ pose estimates is poor, but results in a good point-cloud overlap, then the matching procedure is deceived. In single object matching scenes, this rarely occurs. In instances of cluttered scenes, there is more opportunity for the model point-cloud to be matched incorrectly in the scene but result in good point-cloud overlap. A different cost metric (one that is not solely determined by point-cloud overlap) may be necessary when using SOUR-CVFH or SOL-CVFH in cluttered scenes.

During the development of both SOUR-CVFH and SOL-CVFH , four distance metrics were analyzed. The best performing metric was the Jaccard distance of Eq. 5.15 and thus selected. Recall that the only disadvantage to the Jaccard distance metric is its susceptibility to data occlusions. Occlusions obviously occur in cluttered scenes resulting in histograms with missing infor- 
mation. For use in cluttered scenes, it may be of importance to reassess the distance metric for matching histograms.

\subsection{Rendezvous with an Asteroid}

\subsubsection{Problem Scenario}

An illustrative example of using SOUR-CVFH and SOL-CVFH for a simple relative navigation with the asteroid Itokawa is presented. This scenario is similar to that of Section 5.1.4 which demonstrated the use of OUR-CVFH . The approach begins at a range of $350 \mathrm{~m}$ and ends at $250 \mathrm{~m}$. Range measurements are taken with a simulated LIDAR of size $128 \times 128$ pixels and a $20^{\circ} \mathrm{FOV}$. Each point of the point-cloud is corrupted by Gaussian noise with a standard deviation of $\sigma=0.05$ meters. Measurements are provided every second $(1 \mathrm{~Hz})$ and are processed with an MEKF. The initial state uncertainty in the MEKF is $25 \mathrm{~m}$ in position, $5 \mathrm{~cm} / \mathrm{s}$ in velocity, $10 \mathrm{deg}$ in attitude, and 0.06 $\mathrm{deg} / \mathrm{sec}$ in body rate. A small Monte Carlo analysis of 50 runs is performed to demonstrate the performance of OUR-CVFH , SOUR-CVFH , SOL-CVFH , and the MEKF.

Database training is performed on the Itokawa model prior to the approach experiment. Training followed the process in Sec. 5.3.2 starting at range of $235.96 \mathrm{~m}$ and increasing for eight steps at $29.49 \mathrm{~m}$ per step. The training polyhedron had 642 vertices with the sensor nadir pointing. Clustering parameters that are common to all methods are $t_{\mathbf{n}}=\cos \left(2^{\circ}\right)$ and $t_{c}=1$ for mean curvature. Additionally, for SOUR-CVFH and SOL-CVFH chosen parameters 
Attitude

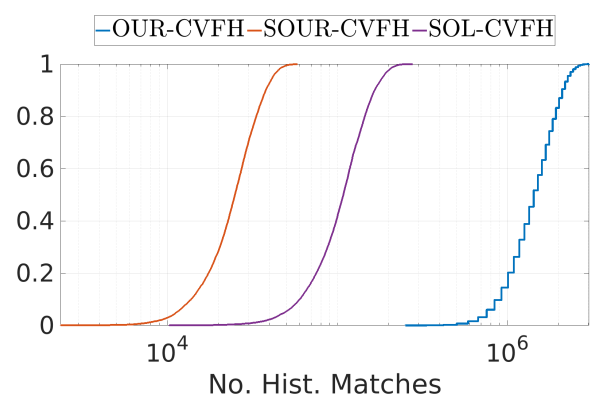

Range

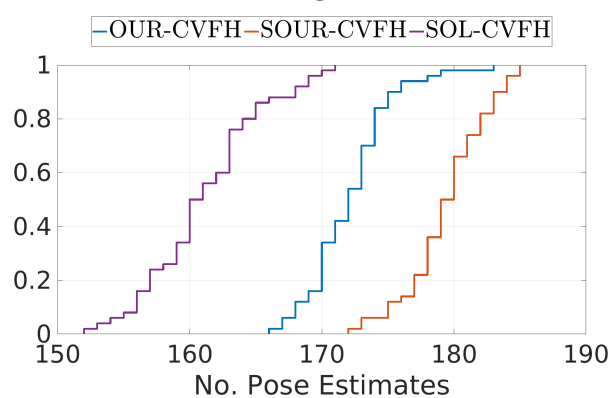

Figure 6.15: CDF plots of (left) Number of histogram matches and (right) number of pose estimates from OUR-CVFH, SOUR-CVFH, and SOL$\mathrm{CVFH}$ for the simulated approach to Itokawa.

are $t_{J}=0.5$ and $t_{\sigma}=0.9$ for both scale ratios. Remaining parameters are set from Table 5.11.

\subsubsection{Unfiltered Pose Performance}

Raw pose estimation results across all 50 Monte Carlo runs are accumulated in in Fig. 6.16, Fig. 6.17, and Fig. 6.18 for OUR-CVFH , SOUR-CVFH , and SOL-CVFH respectively. The varying pose estimation accuracy is affected by observability of the object. Using feature histograms for pose estimation, Itokawa is known to have an observability issue at various poses [16]. The issue does not cause problems for the navigation filter. Fig. 6.15 shows CDF plots of the number of histogram matches and number of pose estimates provided by each of the methods. As expected, SOUR-CVFH requires the least number of histogram matches while returning the most pose estimates. 

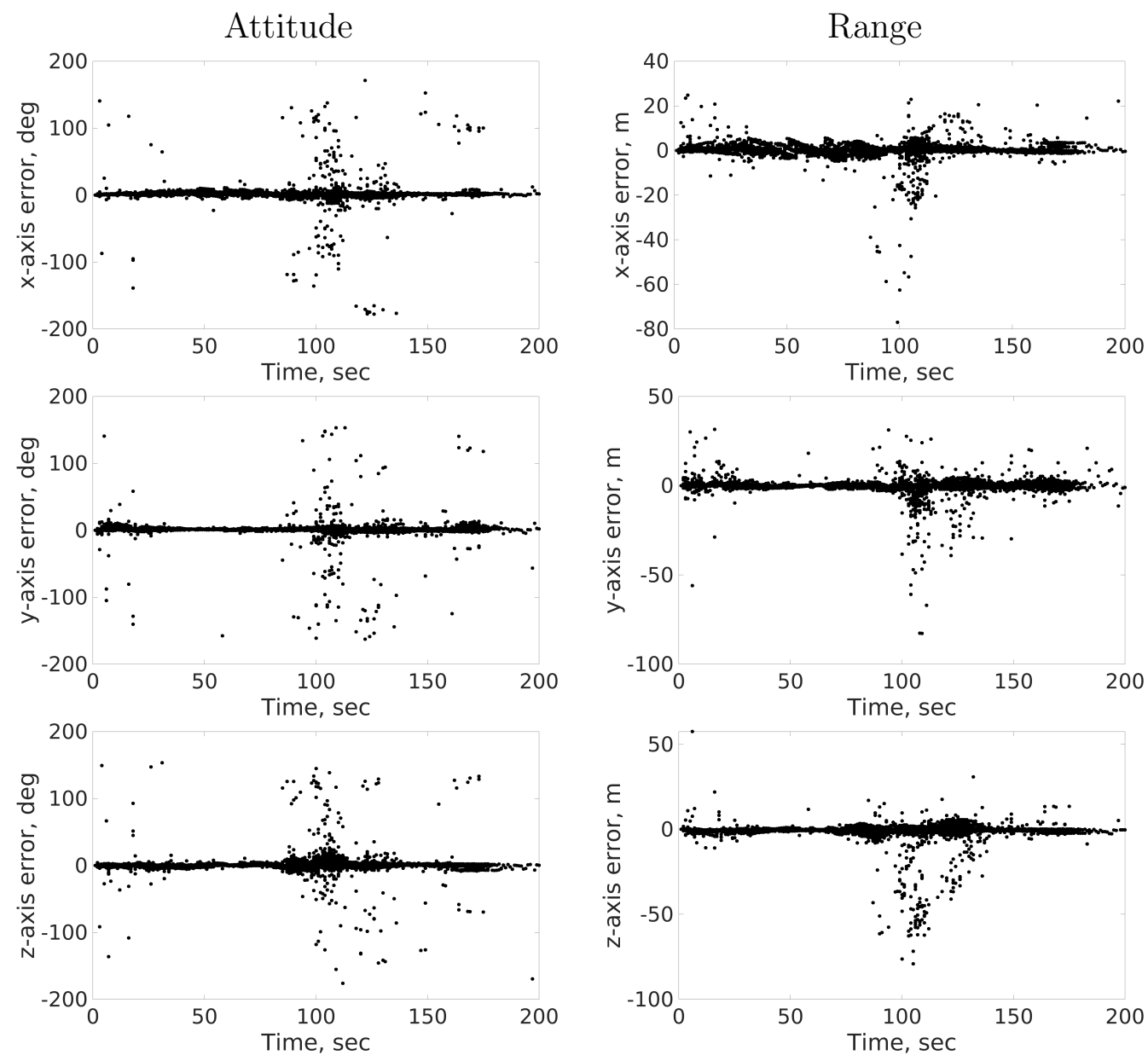

Figure 6.16: Unfiltered pose estimates from OUR-CVFH for the simulated approach to Itokawa. 

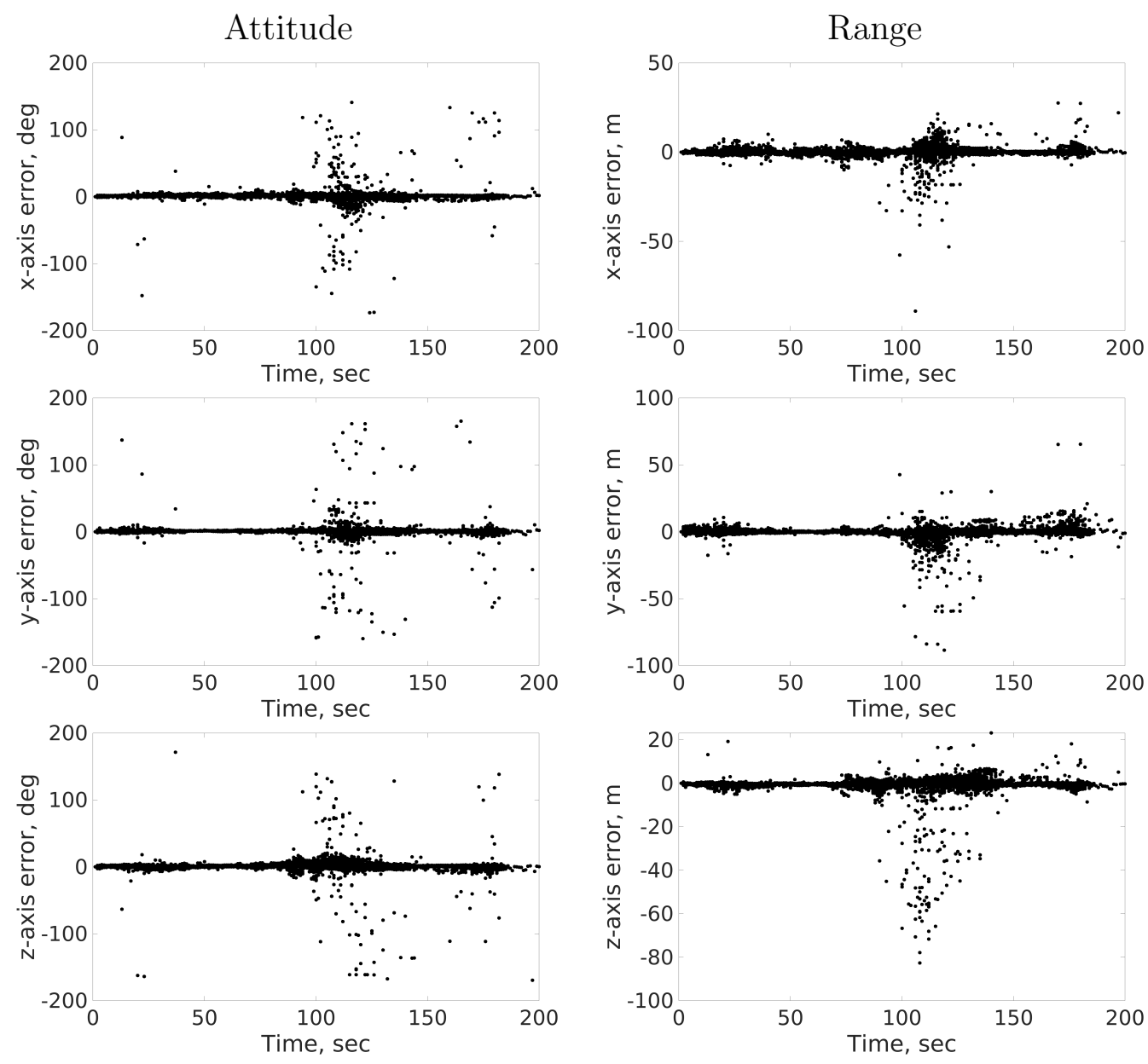

Figure 6.17: Unfiltered pose estimates from SOUR-CVFH for the simulated approach to Itokawa. 

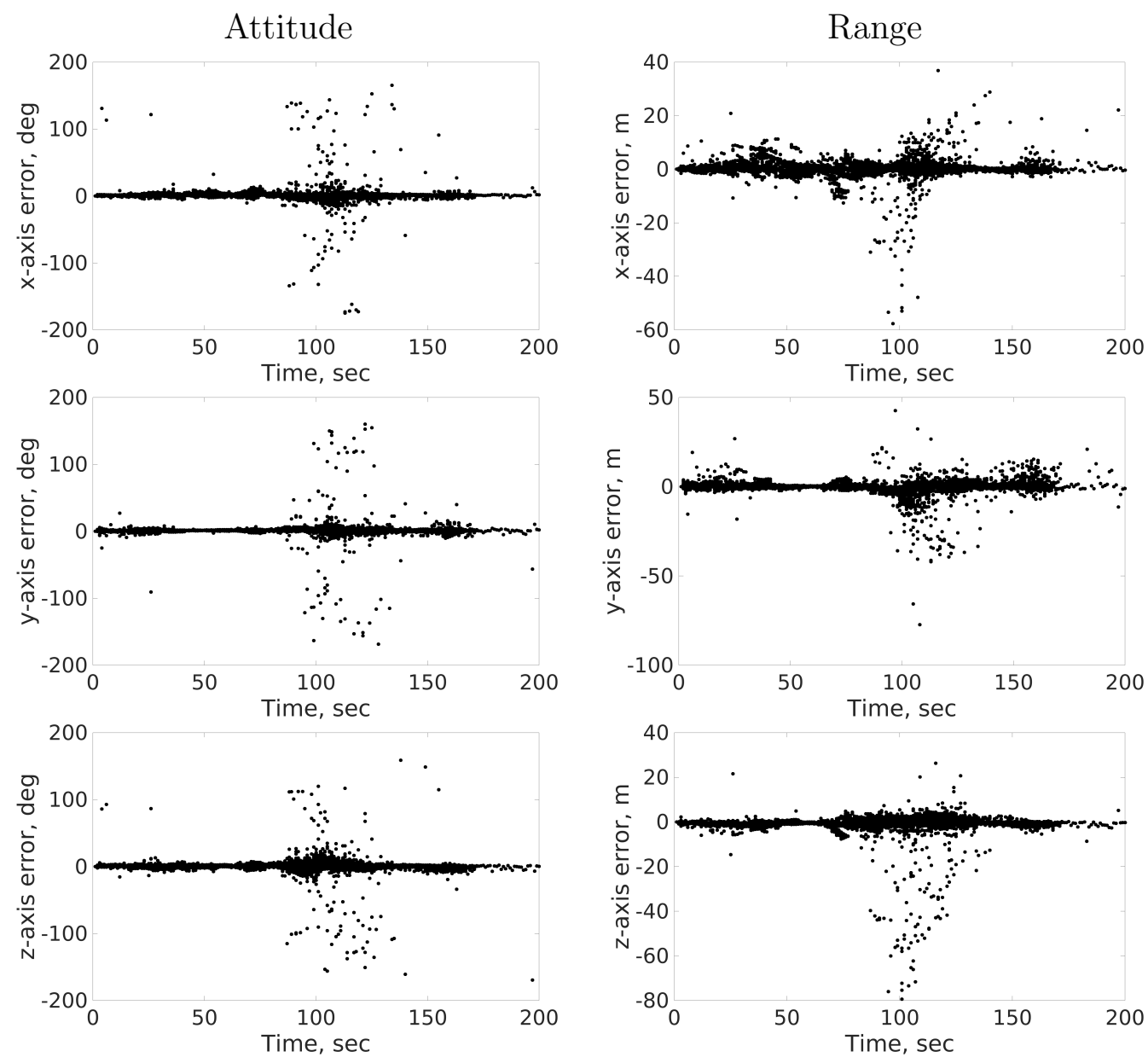

Figure 6.18: Unfiltered pose estimates from SOL-CVFH for the simulated approach to Itokawa. 


\subsubsection{Filtered Pose Performance}

A simply navigation filter in the form of an MEKF is constructed as defined in Sec. 5.1.4.3. Results for filtered relative attitude, relative position, relative velocity, and relative body rate are shown in Fig. 6.19, Fig. 6.20, and Fig. 6.21 for OUR-CVFH, SOUR-CVFH , and SOL-CVFH respectively. Despite the temporary unobservability with the unfiltered pose estimates, the MEKF still performs well. These results confirm that SOUR-CVFH and SOLCVFH may be use in RelNav applications. These methods offer an independent approach to pose estimation that is separate from the navigation filter. 

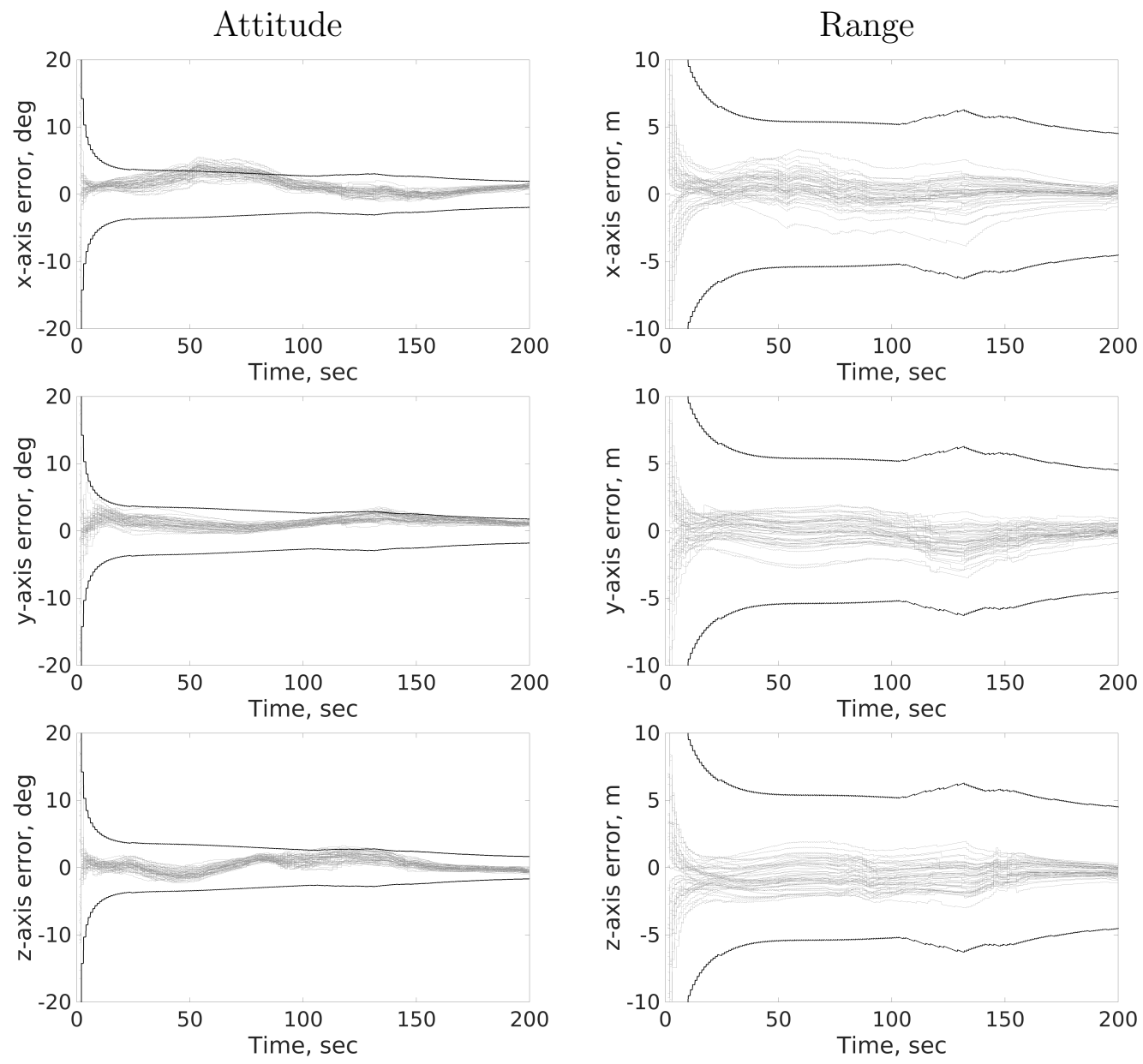

Figure 6.19: MEKF filtered attitude (left) and position (right) estimates from OUR-CVFH for the simulated approach to Itokawa. 

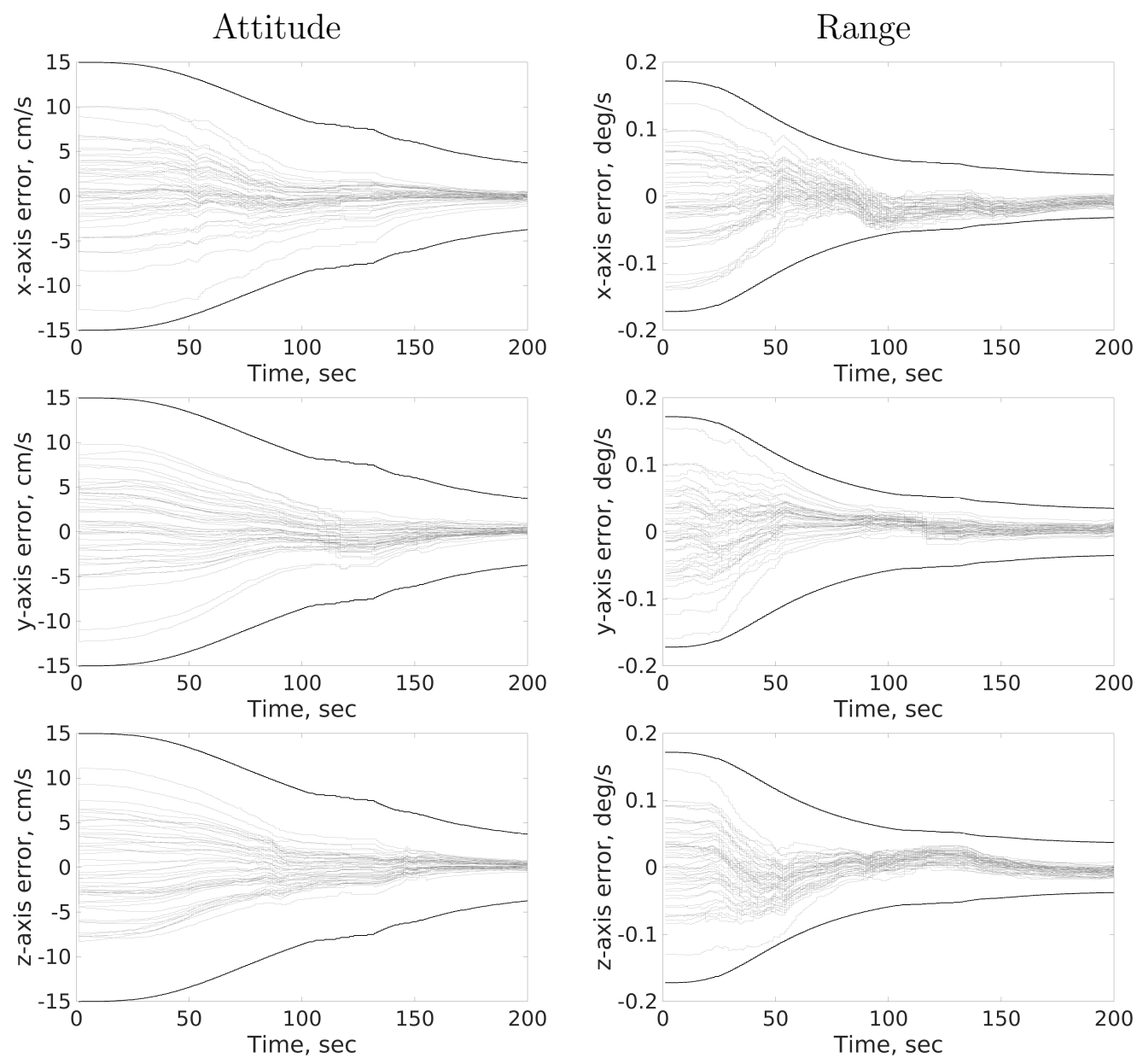

Figure 6.19: Continued. MEKF filtered velocity (left) and attitude rate (right) for OUR-CVFH 

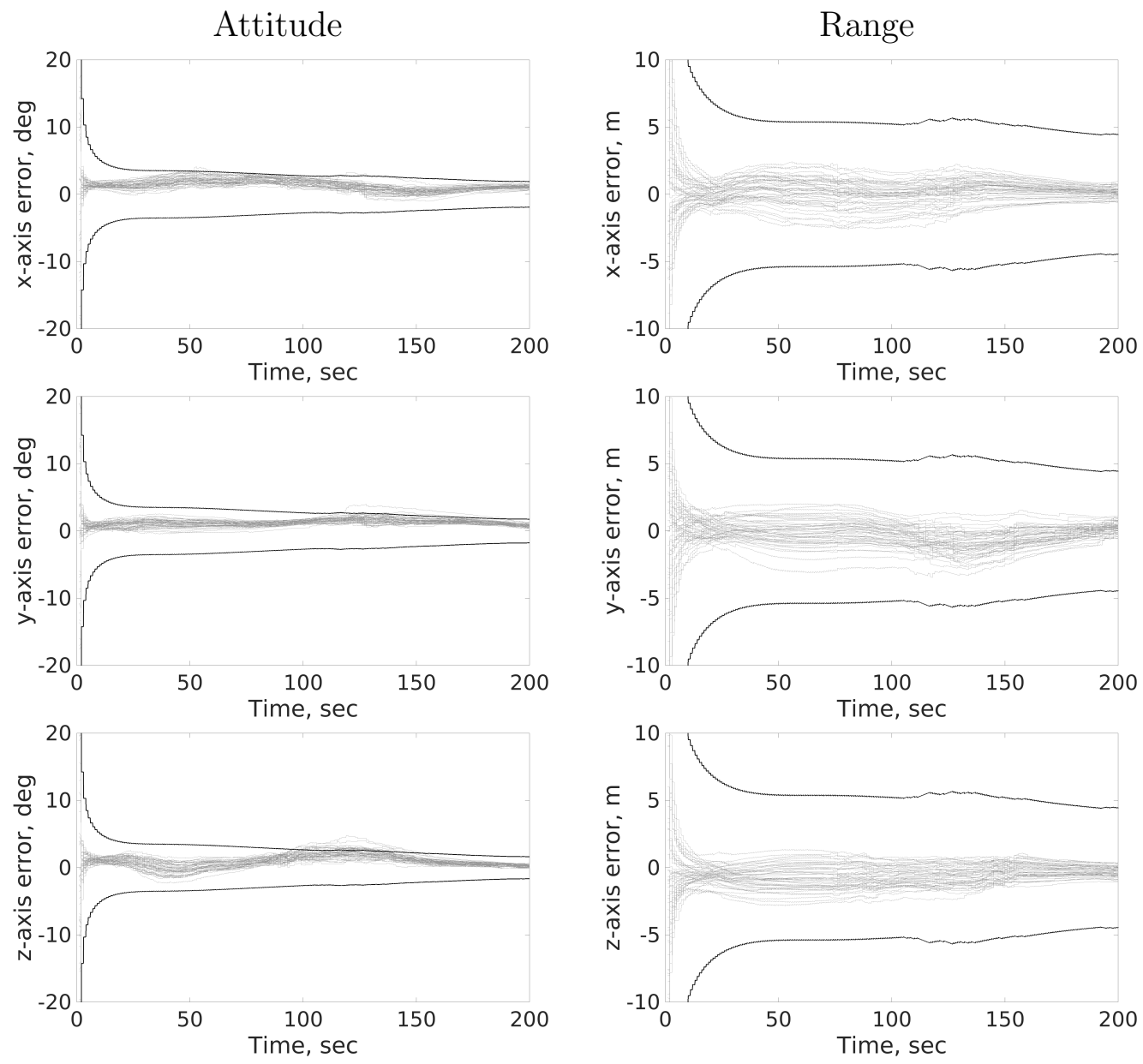

Figure 6.20: MEKF filtered attitude (left) and position (right) estimates from SOUR-CVFH for the simulated approach to Itokawa. 

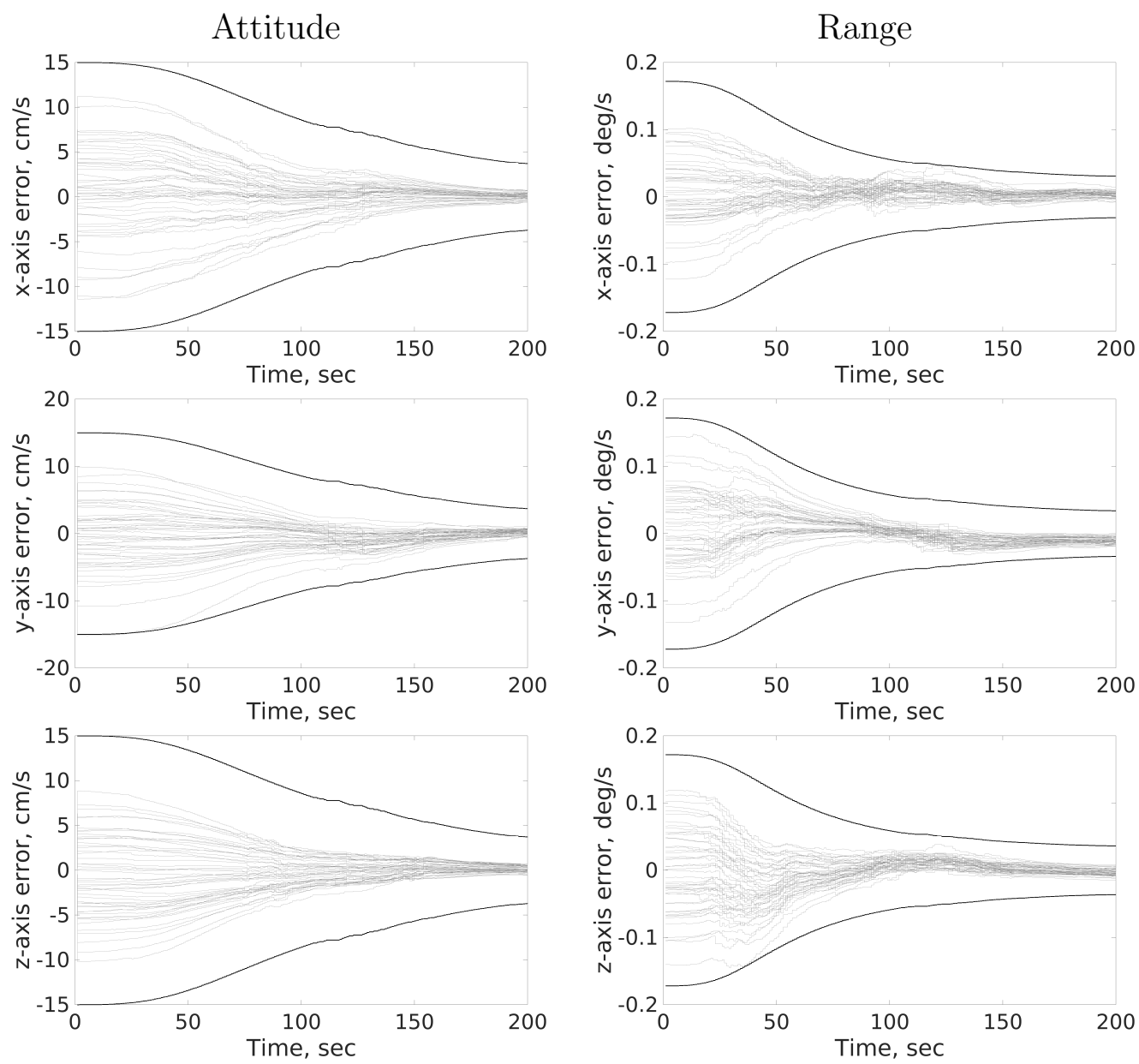

Figure 6.20: Continued. MEKF filtered velocity (left) and attitude rate (right) for SOUR-CVFH 

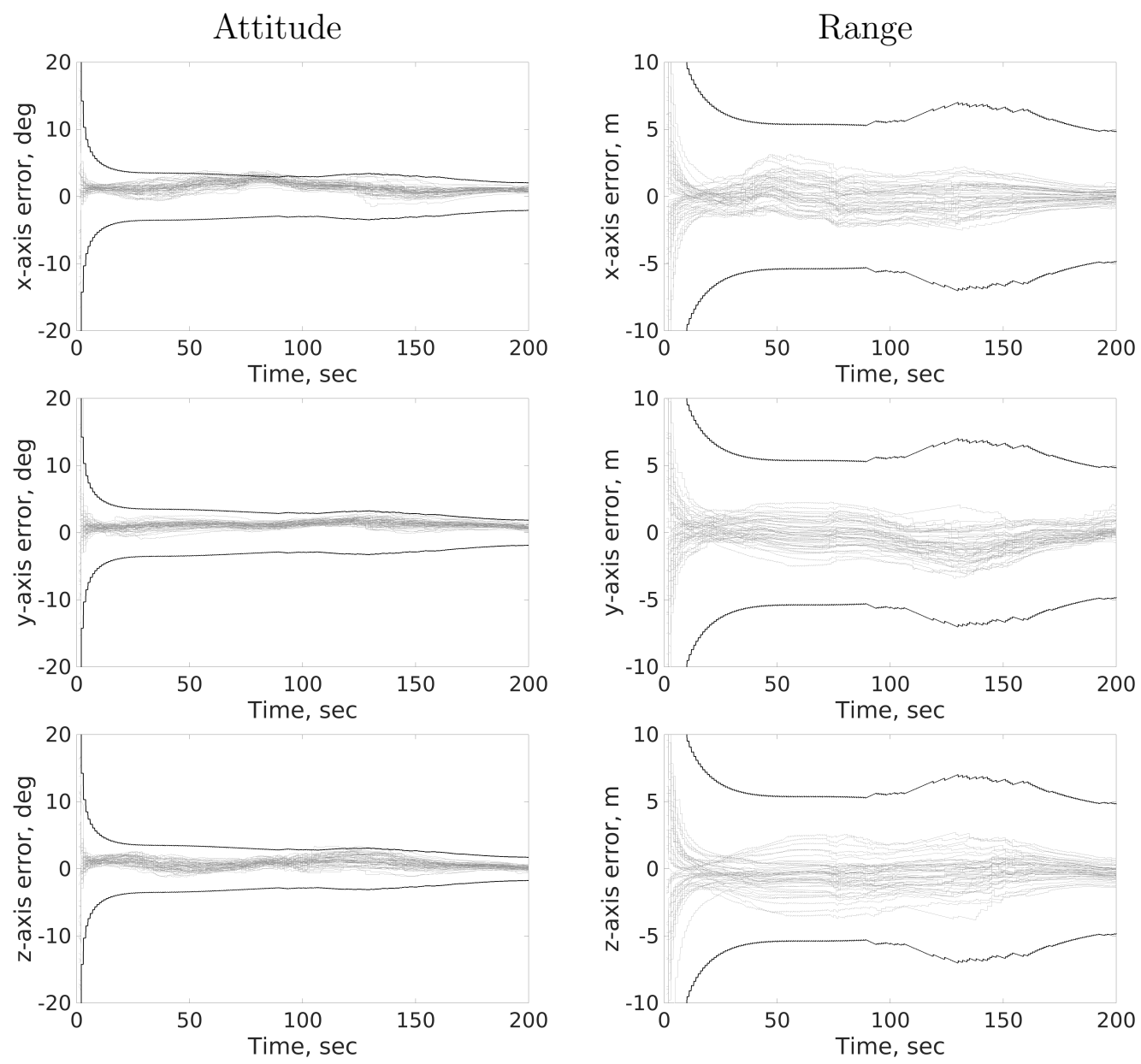

Figure 6.21: MEKF filtered attitude (left) and position (right) estimates from SOL-CVFH for the simulated approach to Itokawa. 

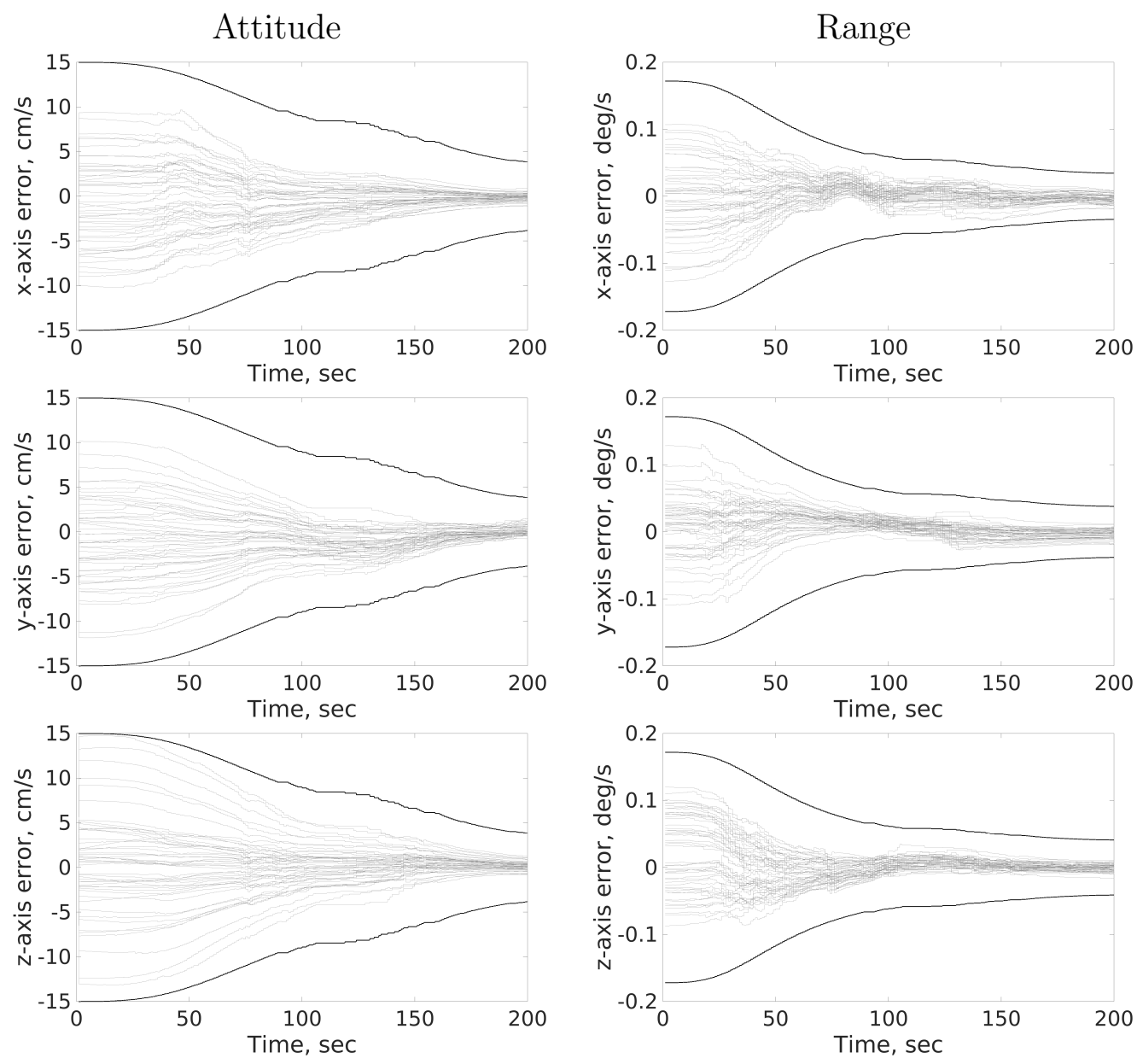

Figure 6.21: Continued. MEKF filtered velocity (left) and attitude rate (right) for SOL-CVFH 


\section{Chapter 7}

\section{Conclusions}

\subsection{Conclusions}

This dissertation presented methods of explicitly utilizing scale in keypoint detection and feature description. Intentionally using scale - which describes size - carries many attributes such as flexible detection in diverse environments and quicker matching procedures. This research offers four main contributions which are discussed individually in the following paragraphs.

Chapter 3 presented methods of signal diffusion on 3D surfaces. A discussion on surface representation types is presented for both explicit and implicit surfaces. Both representations may use the diffusion kernels of either the Laplace-Beltrami operator (LBO) or the Gaussian. Three discrete constructions of the LBO are considered: umbrella, cotangent, and the recent mesh LBO. Both geodesic and Euclidean distance metrics are compared for the discrete Gaussian. It was found that explicit surfaces are less computationally expensive than implicit surfaces, and that the Gaussian is less computationally expensive than the LBOs. Examples for diffusion of various signals on surfaces are presented and visually compared to the well-studied 2D Gaussian diffusion on images. Development of signal diffusion methods on 
3D surfaces allow for the design of scale-space construction on 3D surfaces, as well as possible applications in visual graphics industries.

Chapter 4 constructs a scale-space on explicit 3D surfaces using diffusion kernels of either the LBO or Gaussian. Of particular importance in scalespace construction is time step selection. Time-steps are selected such that each scale-space level is separated by an constant ratio. The main contribution of this chapter is to specifically select the ratio such that the diffusion kernel is only calculated once, resulting in computational efficiency. Discretized diffusion with an LBO (i.e. method DE) is implemented with backward Euler (which is unconditionally stable for any size step), thus the time steps may be of arbitrary size provided that the specified scale-space levels are calculated. Gaussian diffusion (i.e. method GE) purposely selects the ratio to coincide with the property that the variance of two convolved Gaussians is the sum of the original variances. These scale-spaces do no downsample or reconstruct the surface in any way. For specific instances of a planar homogeneously connected surface, the developed scale-spaces simplify to the scale-space presented by SIFT [49]. It was found that discretized diffusion with the mesh LBO (i.e. method DE) performs best on keypoint repeatability metrics as compared to the other LBOs and Gaussian diffusion (i.e. method GE).

Chapter 5 amalgamates scale with a popular feature descriptor to develop the Scaled Oriented Unique Repeatable Clustered Viewpoint Feature Histogram (SOUR-CVFH ). Several small modifications are made to OURCVFH to increase its robustness to noisy measurements and explicitly use 
scale in the histogram matching procedure yielding SOUR-CVFH . Notable alterations include (1) linear interpolation of the angular features, (2) description space selection of angular features, (3) comparing cluster scales in the matching process, and (4) simplifying the spatial feature interpolation process. Each step of the process presents pose estimation errors to determine the modifications' effects. A variant of SOUR-CVFH is also developed called Scaled and Oriented Locally CVFH (SOL-CVFH ) that defines its reference frame solely on the cluster instead of the entire scene. An experiment comparing various combinations of features shows that in some situations, a smaller feature descriptor may result in more accurate pose estimates. Explicitly using scale in feature descriptors quickens the matching procedure and improves pose estimation.

Chapter 6 demonstrates the use of SOUR-CVFH and SOL-CVFH in multiple scenarios. The first experiment is individual pose estimation accuracy for five models. It demonstrates that SOUR-CVFH and SOL-CVFH offer improved pose estimation performance over OUR-CVFH . SOUR-CVFH requires up to two orders of magnitude fewer costly histogram matches than OUR-CVFH, where SOL-CVFH performs intermediately. SOUR-CVFH has a bounded cost metric that returns more estimates than OUR-CVFH . The second experiment is object detection and pose estimation in cluttered scenes using the SHOT Dataset 3 [2]. Despite breaking the SOUR-CVFH assumption (inherited from OUR-CVFH ) of a segmented scene, SOUR-CVFH performs similar to SOL-CVFH (which does not carry the assumption). SOUR- 
CVFH and SOL-CVFH correctly match objects in more than half of the scenes, where OUR-CVFH only succeeds once. This experiment demonstrates that the algorithms work with real data. The third experiment demonstrates the use of SOUR-CVFH and SOL-CVFH in a simulated approach to the asteroid Itokawa. Results indicate that SOUR-CVFH offers superior performance over OUR-CVFH and SOL-CVFH . The purpose of using an independent pose estimation technique is to separate the feedback loop between navigation filter and ICP initialization. This objective is efficiently satisfied with using feature histograms like SOUR-CVFH .

\subsection{Future Work}

\subsubsection{Multi-Scale Framework for 3D Meshes}

This dissertation presented methods of constructing a scale-space for signals on 3D meshes using either of two diffusion kernels: Gaussian and Laplace-Beltrami operator. The conclusion is that these diffusion kernels satisfy the set of scale-space axioms [42], but yield computationally expensive methods. Ultimately, these methods may not be able to perform in real-time scenarios due to the large (and occasionally dense) measured scenes and corresponding kernels. The guarantee of satisfying the scale-space axioms may not be necessary.

Multi-scale frameworks are generalization of scale-space operators that offer desirably properties and computationally efficiency, but do not necessary completely satisfy the scale-space axioms. There are many examples of 
multi-scale frameworks on 3D meshes [146, 68, 147, 148]. These methods still examine the object at various scales to find scale-defined clusters or keypoints, but often pre-define the scales of interest. Given the limitations of a full scalespace framework operating in real-time, it would be advantageous to develop a multi-scale framework that automatically determines the scales at which to examine.

\subsubsection{Incorporate Scale into Random Keypoint Feature Descriptors}

Feature detection may be performed with a variety of techniques depending on the desired outcome. Chapters 3 and 4 developed detectors to identify keypoints in scale-space. The development of SOUR-CVFH and SOLCVFH in Chapter 5 detect features as disjoint clustered sets. Another detector method exhibiting promising results detects randomly sampled keypoints from the measured point-cloud $[74,77,149,88]$. The concept being that much of the scene information is repetitive and correlated such that a random selection of points is sufficient for further description. Contrary to the scale-space and cluster detectors, this detection is extremely quick leaving more time and computation for the description process.

Scale-space and cluster feature detectors automatically adjust the support region over which description is performed. Their application in scenarios of mixed-scaled objects or large dynamic-range is robust due to this flexibility. Contrarily, random keypoint detectors often predefine the support region leaving them susceptible to scenarios outside the environments physical as- 
sumptions. Of all the random keypoint detectors, only Spin Images has been extended to a multi-scale framework [150], while others use a scale-space detector with Spin Image descriptors [67].

Merging the benefits of random keypoint detectors with scale-informed description would offer many advantages. First, random keypoint detection would make scale-informed description available for real-time applications. Second, scale-informed description would increase the robustness of random keypoint detection in scenarios with mixed-scale objects. Therefore, a future research path is to amalgamate random keypoint detectors with scaledependent descriptors.

\subsubsection{Geometric Consistency of Clustered Features}

Clustered feature detectors such as SOUR-CVFH and SOL-CVFH from Chapter 5 only utilize one cluster at a time to estimate pose. It is achievable because of a fully defined 6 DOF reference frame fixed to the cluster. Random keypoint detectors often lack a fully defined reference frame, opting for a reduced 5 DOF reference frame [74, 77]. Pose estimation with partial reference frames is achieved using geometric consistency of multiple keypoints to constrain the undefined parameters. This process not only calculated the 6 DOF pose, but also includes information distributed throughout the scene.

While feature detectors with fully defined 6 DOF reference frames do not require multiple keypoints for pose estimation, the use of geometric consistency would benefit the process. First, keypoints identified at the far end of a 
scene may bias the pose estimation, but including keypoints from throughout the scene (especially from both far ends of the scene) could limit and offset the potential bias. Second, geometric consistency could eliminate a poor proposed pose estimate simply because of inconsistency with other clusters' relative poses. Third, cluttered scenes reduce probability of seeing large clusters but many small clusters may be present. Therefore, a future research direction is to develop a process of examining geometric consistency of multiple features with fully defined 6 DOF reference frames.

\subsubsection{Inclusion of Scene Color Cues with SOUR-CVFH and SOL- CVFH}

On the advent of 3D sensors, only range information was available, leading to many feature descriptors based on shaped only [151, 60, 152, 74, 153, 76, 77, 78, 79, 54, 154, 149]. Many modern 3D sensors return both color and range information in an RGB-D format, motivating the use of both color and shape in keypoint detection and description. Literature contains fewer examples of detectors and descriptors using both shape and color sources $[155$, $68,156,88,62]$.

Descriptors incorporating information from both sources can recognize objects indistinct in either format. Examples include a patterned soda can (i.e. indistinct in shape, informative in color) or a solid blue rock (e.g. informative in shape, indistinct in color). Shape and color descriptors tend to have larger dimensions due to the increased description space such as the color 
informed OUR-CVFH with 1327 bins [156] compared to the 303 bins of OURCVFH [54]. This often increases the time required for matching and hypothesis verification steps, but also improves object recognition performance.

The scale-space developed in Chapters 3 and 4 is for any signal defined on the surface. Color was not explicitly tested in this dissertation, but is a direction of future research. Similarly, Chapter 5 developed SOUR-CVFH and SOL-CVFH that do not characterize color. The reduced run-time of SOURCVFH and SOL-CVFH - due to the scale matching procedure - may permit the inclusion of color into the histogram descriptor without significant affects on performance time.

\subsubsection{Multi-Feature Combinations}

An abundance of point-cloud feature descriptors exist in literature [157, 158, 159, 160]. May are reportedly generic enough for a variety of scenarios, but most detectors and descriptors are tuned for specific applications. There has been little investigation into descriptor selection depending on the application. Occasionally, descriptors specifically designed for a particular detector do not offer the best results, whereas cross-combinations of different publications may offer improvements [3].

With such availability of point-cloud feature descriptors, preselecting one for the application at hand may limit capabilities. Instead, it may be useful to construct a bag-of-features containing multiple detector and description methods to be used simultaneously or intermittently. Quicker, but less 
accurate, methods may be used for every measurement alongside the slower, but more accurate, methods offering intermittent assurance. A bag-of-features would be beneficial in situations where the best performing detectors and descriptors change during a mission. A future research direction is to test the pose estimation and time performance of a bag-of-features. 


\section{Bibliography}

[1] C. B. MacDonald and S. J. Ruuth, "The implicit closest point method for the numerical solution of partial differential equations on surfaces," SIAM Journal of Scientific Computing, vol. 31, no. 6, pp. 4330-4350, 2009.

[2] F. Tombari, S. Salti, and L. D. Stefano, "Shot: Unique signatures of histograms for local surface description." www.vision.deis.unibo. it/research/80-shot.

[3] S. Salti, A. Petrelli, F. Tombari, and L. D. Stefano, "On the affinity between 3d detectors and descriptors," in International Conference on 3D Imaging, Modeling, Processing, Visualization Transmission, October 2012.

[4] S. Foix, G. Alenyà, and C. Torras, "Lock-in Time-of-Flight (ToF) Cameras: A Survey," IEEE Sensors Journal, vol. 11, no. 9, pp. 1917-1926, 2011.

[5] C. D. Mutoi, P. Zanuttigh, and G. Cortelazzo, Time-of-Flight Cameras and Microsoft Kinect. New York, NY: Springer, 2012.

[6] Z. Zhang, "Microsoft kinect sensor and its effect," IEEE Autonomy, vol. 190 , pp. 4-10, 2012. 
[7] J. A. Christian and S. Cryan, "A survey of lidar technology and its use in spacecraft relative navigation," in AIAA Guidance, Navigation, and Control (GNC) Conference, 2013.

[8] L. Zhang, B. Curless, and S. M. Seitz, "Spacetime stereo: shape recovery for dynamic scenes," in IEEE Conference Computer Vision and Pattern Recognition (CVPR), 2003.

[9] J. Davis, R. Ramamoorthi, and S. Rusinkiewics, "Spacetime stereo: A unifying framework for depth from triangulation," in IEEE Conference on Computer Vision and Pattern Recognition (CVPR), June 2003.

[10] R. B. Rusu and S. Cousins, "3D is here: Point Cloud Library (PCL)," in IEEE International Conference on Robotics and Automation (ICRA), May 9-13 2011.

[11] G. Bradski, "The OpenCV Library," Dr. Dobb's Journal of Software Tools, 2000.

[12] P. Cignoni, M. Callieri, M. Corsini, M. Dellepiane, F. Ganovelli, and G. Ranzuglia, "MeshLab: an Open-Source Mesh Processing Tool," in Eurographics Italian Chapter Conference (V. Scarano, R. D. Chiara, and U. Erra, eds.), The Eurographics Association, 2008.

[13] J. O. Woods and J. A. Christian, "GLIDAR: An OpenGL-based, RealTime, and Open Source 3D Sensor Simulator for Testing Comuter Vision Algorithms," Journal of Imaging, vol. 2, January 2016. 
[14] Y. Guo, M. Bennamoun, F. Sohel, M. Lu, and J. Wan, "3D Object Recognition in Cluttered Scenes with Local Surface Features: A Survey," IEEE Transactions on Pattern Analysis and Machine Intelligence, vol. 36, no. 11, pp. 2270-2287, 2014.

[15] Y. Guo, M. Bennamoun, F. Sohel, M. Lu, J. Wan, and N. Kwok, "A Comprehensive Performance Evaluation of 3D Local Feature Descriptors," International Journal of Computer Vision, vol. 116, no. 1, pp. 66$89,2015$.

[16] A. P. Rhodes, J. A. Christian, and T. Evans, "A concise guide to feature histograms with applications to lidar-based spacecraft relative navigation," Journal of Astronautical Sciences, vol. 64, pp. 414-445, 2017.

[17] B. Höfle, "Radiometric correction of terrestrial lidar point cloud data for individual maize plant detection," IEEE Geoscience and Remote Sensing Letters, vol. 11, January 2014.

[18] R. B. Rusu, Z. C. Marton, N. Blodow, M. Dolha, and M. Beetz, "Towards 3D Point Cloud Based Object Maps for Household Environments," Journal of Robotics and Autonomous Systems, vol. 56, pp. 927-941, August 2008.

[19] J. O. Woods, J. A. Christian, and T. A. Evans, "A 6-DOF Pose Initialization Strategy for LIDAR-Based Non-Cooperative Navigation," in 38th AAS Guidance, Navigation, and Control Conference, January-February 2015. 
[20] J. Levinson, J. Askeland, J. Becker, J. Dolson, D. Held, S. Kammel, J. Z. Kolter, D. Langer, O. Pink, V. Pratt, M. Sokolsky, G. Stanek, D. Stavens, A. Teichman, M. Werling, and S. Thrun, "Towards fully autonomous driving: Systems and algorithms," in IEEE Intelligent Vehicle Symposium (IV), June 2011.

[21] D. Woffinden and D. Geller, "Observability Criteria for Angles-Only Navigation," IEEE Transactions on Aerospace and Electronic Systems, vol. 45, no. 3, pp. 1194-1208, 2009.

[22] B. Naasz and M. Moreau, "Autonomous RPOD Challenges for the Coming Decade," in 35th Annual AAS Guidance and Control Conference, (Breckenridge, CO), 3-8 Feb 2012.

[23] J. Christian, S. Robinson, C. D'Souza, and J. Ruiz, "Cooperative Relative Navigation of Spacecraft Using Flash Light Detection and Ranging Sensors," Journal of Guidance, Control, and Dynamics, vol. 37, no. 2, pp. $452-465,2014$.

[24] S. Robinson and J. Christian, "Pattern Design for 3D Point Matching," NAVIGATION: Journal of The Institute of Navigation, vol. 62, no. 3, pp. 189-203, 2015.

[25] R. Rohrschneider, W. Tandy, J. Bladt, and I. Gravseth, "Simulation Results of ISS AR\&D Using Only Range Images," in AIAA Guidance, Navigation, and Control Conference, (Minneapolis, MN), 13-16 August 2012 . 
[26] J. Galante, J. Van Eepoel, M. Strube, N. Gill, M. Gonzalez, A. Hyslop, and B. Patrick, "Pose Measurement Performance of the Argon Relative Navigation Sensor Suite in Simulated Flight Conditions," in AIAA Guidance, Navigation, and Control Conference, 2012.

[27] J. Sell, A. P. Rhodes, J. O. Woods, , J. A. Christian, and T. Evans, "Pose performance of lidar-based navigation for satellite servicing," in AIAA Astrodynamics Specialist Conference, August 2014.

[28] R. Opromolla, G. Fasano, G. Rufino, and M. Grassi, "Uncooperative Pose Estimation with a LIDAR-Based System," Acta Astronautica, vol. 110, pp. 287-297, 2015.

[29] R. Opromolla, G. Fasano, G. Rufino, and M. Grassi, "A Model-Based 3D Template Matching Technique for Pose Acquisition of an Uncooperative Space Object," Sensors, vol. 15, no. 3, pp. 6360-6382, 2015.

[30] J. O. Woods and J. A. Christian, "LIDAR-based Relative Navigation with respect to Non-cooperative Objects," Acta Astronautica, 2016.

[31] P. J. Besl and N. D. McKay, "A Method for Registration of 3-D Shapes," IEEE Transaction on Pattern Analysis and Machine Intelligence, vol. 14, no. 2, pp. 239-256, 1992.

[32] Y. Chen and G. Medioni, "Object Modeling by Registratoin of Multiple Range Images," IEEE International Conference on Robotics and Automation, pp. 2724-2729, 1991. 
[33] S. Billings and R. Taylor, "Generalized iterative most likely oriented point registration," International Journal of Computer Assisted Radiology and Surgery (J-CARS), pp. 1213-1226, 2015.

[34] A. V. Segal, D. Haehnel, and S. Thrun, "Generalized icp," in Robotics: Science and Systems, June 2009.

[35] S. Rusinkiewicz and M. Levoy, "Efficient variants of the icp algorithm," in International Conference on 3D Digital Imaging and Modeling, 2001.

[36] J. Yang and D. Campbell, "Go-icp: A globally optimal solution to 3d icp point-set registration," IEEE Transactions on Pattern Analysis and Machine Intelligence, vol. 38, pp. 2241-2254, November 2016.

[37] J. Straub, T. Campbell, J. P. How, and J. W. F. III, "Efficient globally optimal point cloud alignment using bayesian nonparametric mixtures," CoRR, 2016.

[38] S. Ruel, T. Luu, M. Anctil, and S. Gagnon, "Target Localization from 3D Data for On-orbit Autonomous Rendezvous and Docking," in IEEE In Aerospace Conference, pp. 1-8, 2008.

[39] R. Opromolla, G. Fasano, G. Rufino, and M. Grassi, "Large Space Debris Pose Acquisition in Close Proximity Operations," IEEE Metrology for Aerospace, pp. 491-496, June 2015. 
[40] A. P. Rhodes, E. Kim, and J. A. Christian, "Lidar-based relative navigation of non-cooperative objects using point cloud descriptors," in AIAA Space 2016, September 2016.

[41] F. Aghili, M. Kuryllo, G. Okouneva, and C. English, "Fault-tolerant pose estimation of space objects," in IEEE/ASME International Conference on Advanced Intelligent Mechatronics, July 2010.

[42] J. Sporring, M. Nielson, L. Florack, and P. Johansen, eds., Gaussian Scale Space Theory, vol. 8. Kluwer Academic Publishers, 1997.

[43] P. R. Martin and U. Grunert, "Gaglion cells in mammalian retinae," in The Visual Neurosciences, volume 1 (J. S. Werner and L. M. Chalupa, eds.), ch. 26, pp. 410-421, MIT Press, 2004.

[44] V. Vonikakis, D. Chrysostomou, R. Kouskouridas, and A. Gasteratos, "A biologically inspired scale-space for illumination invariant feature detection," Measurement Science and Technology, vol. 24, June 2013.

[45] K. Mikolajczyk and C. Schmid, "Indexing based on scale invariant interest points," in Eighth IEEE International Conference on Computer Vision, July 2001.

[46] T. Kadir and MichaelBrady, "Saliency, scale and image description," International Journal of Computer Vision, vol. 45, pp. 83-105, November 2001. 
[47] K. Mikolajczyk and C. Schmid, "Scale and affine invariant interest point detectors," International Journal of Computer Vision, vol. 60, no. 1, pp. 63-86, 2004.

[48] F. Jurie and C. Schmid, "Scale-invariant shape features for recognition of object categories," in IEEE Conference on Computer Vision and Pattern Recognition (CVPR), June 27 - July 22004.

[49] D. G. Lowe, "Distinctive image features from scale-invariant keypoints," International Journal of Computer Vision, vol. 60, pp. 91-110, 2004.

[50] H. Bay, T. Tutelaars, and L. V. Gool, "Surf: Speeded up robust features," Computer Vision and Image Understanding, vol. 3, no. 10, pp. 346$359,2008$.

[51] T. Lindeberg, "Image matching using generalized scale-space interest points," J Math Imaging Vision, vol. 52, pp. 3-36, 2015.

[52] T. Lindeberg, "Scale-space theory: A basic tool for analyzing structures at different scales," Journal of Applied Statistics, vol. 2, no. 21, pp. 224270, 1994.

[53] P. Viola and M. Jones, "Rapid object detection using a boosted cascade of simple features," in IEEE Conference on Computer Vision and Pattern Recognition (CVPR), December 2001.

[54] A. Aldoma, F. Tombari, R. B. Rusu, and M. Vincze, "Our-cvfh - oriented, unique and repeatable clustered viewpoint feature histograms for 
object recognition and 6dof pose estimation," Pattern Recognition: Joint 34th DAGM and 36th OAGM Symposium, pp. 113-122, August 2012.

[55] T. Iijima, "Basic theory on normalization of a pattern (in case of onedimensional pattern)," in Bulletin of Electrical Laboratory, vol. 26, pp. 368388, 1962. In Japanese.

[56] N. Otsu, Mathematical Studies on Feature Extraction in Pattern Recognition. Ph.d., Researches of the Electotechnical Laboratory, 1981. In Japanese.

[57] H. Fadaifard, G. Wolberg, and R. Haralick, "Multiscale 3d feature extraction and matching with an application to $3 \mathrm{~d}$ face recognition," Graphical Models, vol. 75, pp. 157-176, 2013.

[58] A. P. Rhodes and J. A. Christian, "Constructing a 3d scale space from implicit surfaces for vision based spacecraft relative navigation," in $A A S$ 41st Annual Guidance and Control Conference, February 2018.

[59] A. P. Rhodes and J. A. Christian, "Scale-space construction and keypoint detection on $3 \mathrm{~d}$ meshes using discrete laplacians," International Journal of Computer Vision, 2019. Submitted to Journal.

[60] P. Baryiha, J. Novatnack, and G. Schwartz, "3d geometric scale variability in range images: Features and descriptors," International Journal of Computer Vision, vol. 99, pp. 232-255, 2012. 
[61] J. Novatnack and K. Nishino, "Scale dependent 3d geometric features," in IEEE Int. Conference on Computer Vision, 2007.

[62] K. P. Cop, P. V. Borges, and R. Dubé, "Delight: An efficient descriptor for global localization using lidar intensities," in IEEE International Conference on Robotics and Automation (ICRA), May 2018.

[63] D. Smeets, J. Keustermans, D. Vandermeulen, and P. Suetens, "meshsift: Local surface features for $3 \mathrm{~d}$ face recognition under expression variations and partial data," Computer Vision and Image Understanding, vol. 117, pp. 158-169, 2013.

[64] L. Kobbelt, S. Campagna, J. Vorsatz, and H.-P. Seidel, "Interactive multi-resolution modeling on arbitrary meshes," in Proceedings of the 25th Annual Conference on Computer Graphics and Interactive Techniques, SIGGRAPH, pp. 105-114, 1998.

[65] M. Schlattmann, P. Degener, and R. Klein, "Scale space based feature point detection on surfaces," Journal of WSCG, vol. 16, January 2008.

[66] A. Zaharescu, E. Boyer, and R. Horaud, "Keypoints and local descriptors of scale functions on $2 \mathrm{~d}$ manifolds," International Journal of Computer Vision, vol. 100, pp. 78-98, May 2012.

[67] T. Hou and H. Qin, "Efficient computation of scale-space features for deformable shape correspondences," in European Conference on Computer 
Vision, (Berlin, Heidelberg), pp. 384-397, Springer Berlin Heidelberg, 2010.

[68] E. R. Smith, R. J. Radke, and C. V. Stewart, "Physical scale keypoint: Matching and registration for combined intensity/range images," International Journal of Computer Vision, vol. 97, no. 2, pp. 2-17, 2012.

[69] G. Zou, J. Hua, M. Dong, and H. Qin, "Surface matching with salient keypoints in geodesic scale space," Computer Animation and Virtual Worlds, vol. 19, pp. 399-410, 2008.

[70] A. Flint, A. Dick, and A. van den Hengel, "Thrift: Local 3d structure recognition," in Digital Image Computing Technique and Applications, 2007.

[71] A. Zaharescu, E. Boyer, K. Varanasi, and R. Horaud, "Surface feature detection and description with applications to mesh matching," in IEEE Conference on Computer Vision and Pattern Recognition (CVPR), pp. 373380, June 2009.

[72] H. Fadaifard and G. Wolberg, "Multiscale 3d feature extraction and matching," in International Conference on 3D Imaging, Modeling, Processing, Visualization and Transmission, May 2011.

[73] E. R. Smith, B. J. King, C. V. Stewart, and R. J. Radke, "Registration of combined range-intensity scans: Initialization through verification," Computer Vision and Understanding, vol. 110, pp. 226-244, May 2008. 
[74] A. E. Johnson, Spin-Images: A Representation for 3D Surface Matching. Ph.d., Carnegie Mellon University, August 1997.

[75] K. Mikolajczyk and C. Schmid, "A performance evaluation of local descriptors," IEEE Transactions on Pattern Analysis and Machine Intelligence, vol. 27, no. 10, pp. 1615-1630, 2005.

[76] R. B. Rusu, Z. C. Maron, N. Blodow, and M. Beetz, "Persistent Point Feature Histograms for 3D Point Clouds," in 10th Int. Conf. Intelligent Autonomous Systems, 2008.

[77] R. B. Rusu, N. Blodow, and M. Beetz, "Fast Point Feature Histograms (FPFH) for 3D Registration," in IEEE Int. Conf. Robotics and Automation, May 2009.

[78] R. B. Rusu, G. B. ad Romain Thibauz, J. Hsu, and W. Garage, "Fast 3D Recognition and Pose Using the Viewpoint Feature Histogram," in IEEE/RSJ Int. Conf. on Intelligent Robots and Systems, October 2010.

[79] A. Aldoma, Markus, Vincze, N. Blodow, D. Gossow, S. Gedikli, R. B. Rusu, G. Bradski, and W. Garage, "CAD-Model Recognition and 6DOF Pose Estimation Using 3D Cues," in IEEE International Conference on Computer Vision Workshops, 2011.

[80] R. B. Rusu, Z. C. Marton, N. Blodow, and M. Beetz, "Learning Information Point Classes for the Acquisition of Object Model Maps," in ICARCV, pp. 643-650, December 2008. 
[81] K. Klasing, D. Althoff, D. Wollherr, and M. Buss, "Comparison of surface normal estimation methods for range sensing applications," in IEEE International Conference on Robotics and Automation, May 2009.

[82] S. Kullback and R. Leibler, "On Information and Sufficiency," The Annals of Mathematical Statistics, vol. 22, pp. 79-86, March 1951.

[83] R. Maronna, D. Martin, and V. Yohai, Robust Statistics: Theory and Methods. West Sussex, England: John Wiley \& Sons, Ltd, 2006.

[84] K. Levenberg, "A Method for the Solution of Certain Non-Linear Problems in Least Squares," Quarterly of Applied Mathematics, pp. 1964-168, 1944.

[85] D. Marquardt, "An Algorithm for Least-Squares Estimation of Nonlinear Parameters," Journal of the Society for Industrial \&3 Applied Mathematics, vol. 11, pp. 431-441, 1963.

[86] R. B. Rusu, A. Holzbach, M. Beetz, and G. Bradski, "Detecting and Segmenting Objects for Mobile Manipulation," in ICCV S3DV Workshops, 2009.

[87] M. K. Agoston, Computer Graphics and Geometric Modelling: Mathematics. Secaucus, NJ, USA: Springer-Verlag New York, Inc., 2005.

[88] S. Salti, F. Tombari, and L. D. Stefano, "Shot: Unique signatures of histograms for surface and texture description," Computer Vision and Image Understanding, vol. 125, pp. 251-264, 2014. 
[89] A. P. Witkin, "Scale space filtering," Proceedings of the Eighth International Joint Conference on Artificial Intelligence, pp. 1019-1022, 1983.

[90] J. Babaud, A. P. Witkina, M. Baudin, and R. O. Duda, "Uniqueness of the gaussian kernel for scale-space filtering," IEEE Transactions on Pattern Analysis and Machine Intelligence, vol. PAMI-8, January 1986.

[91] P. W. Jones, M. Maggioni, and R. Schul, "Manifold parametrizations by eigenfunctions of the laplacian and heat kernels," PNAS, vol. 105, pp. 1803-1808, February 2008.

[92] F. Mokhtarian and A. K. Mackworth, "A theory of multiscale, curvaturebased shape representation for planar curves," IEEE Transactions on Pattern Analysis and Machine Intelligence (TPAMI), vol. 14, pp. 789805, August 1992.

[93] R. Thibeault, L. Hong, C. Hollenberg, and J. Christian, "Triangles vs. quadrilaterals: Selecting the right 3d model format for space science and exploration," in 2nd RPI Space Imaging Workshop, October 2019.

[94] M. Bertalmío, L.-T. Cheng, S. Osher, and G. Sapiro, "Variational problems and partial differential equations on implicit surfaces," Journal of Computational Physics, vol. 174, pp. 759-780, 2001.

[95] J. B. Greer, "An improvement of a recent eulerian method for solving pde's on general geometries," Journal of Scientific Computing, vol. 29, pp. 321-352, December 2006. 
[96] S. J. Ruuth and B. Merriman, "A simple embedding method for solving partial differential equations on surfaces," Journal of Computational Physics, vol. 227, pp. 1943-1961, 2008.

[97] T. März and C. B. Macdonald, "Calculus on surfaces with general closest point functions," SIAM Journal on Numerical Analysis, vol. 50, February 2012 .

[98] J. Berrut and L. Trefethen, "Barycentric lagrange interpolation," SIAM Rev., vol. 46, pp. 501-517, 2004.

[99] L. Trefethen and D. B. III, Numerical Linear Algebra. SIAM, 1997.

[100] X.-D. Liu, S. Osher, and T. Chan, "Weighted essentially non-oscillatory schemes," Journal of Computational Physics, vol. 115, pp. 200-212, November 1994.

[101] G.-S. Jiang and C.-W. Shu, "Efficient implementation of weighted eno schemes," Journal of Computational Physics, vol. 126, pp. 202-228, 1996.

[102] B. Merriman and S. Ruuth, "Diffusion generated motion of curves on surfaces," Journal of Computational Physics, vol. 225, pp. 2267-2282, 082007.

[103] M. Brown, "A Generalized Error Function In n-Dimensions," technical memorandum, U.S. Navel Missile Center, April 1963. NMC Tech- 
nical Memorandum NMC-TM-63-8, http://www.dtic.mil/dtic/tr/ fulltext/u2/401722.pdf.

[104] M. Desbrun, M. Meyer, P. Schröder, and A. H. Barr, "Implicit fairing of irregular meshes using diffusion and curvature flow," in SIGGRAPH 99 Conference Proceedings, pp. 317-324, 1999.

[105] G. Taubin, "A signal processing approach to fair surface design," in SIGGRAPH 95 Conference Proceedings, pp. 351-358, August 1995.

[106] C. Moler and C. V. Loan, "Nineteen dubious ways to compute the exponential of a matrix, twenty-five years later," SIAM Review, vol. 45, February 2003.

[107] A. H. Al-Mohy and N. J. Higham, "A new scaling and squaring algorithm for the matrix exponential," SIAM Journal of Matrix Analysis and Applications, vol. 31, pp. 970-989, August 2009.

[108] N. J. Higham, "The scaling and squaring method for the matrix exponential revisited," SIAM Journal of Matrix Analysis and Applications, vol. 26, pp. 1179-1193, June 2005.

[109] R. B. Sidje, "Expokit: A software package for computing matrix exponentials," ACM Transactions on Mathematical Software, vol. 24, pp. 130156, August 1998.

[110] J. Niesen and W. Wright, "Algorithm 919: A krylov subspace algorithm for evaluating the phi-functions appearing in exponential integrators," 
ACM Transactions on Mathematical Software, vol. 38, pp. 1-19, April 2012.

[111] M. Caliari, M. Vianello, and L. Bergamaschi, "Interpolating discrete advection-diffusion propagators at leja sequences," Journal of Computational and Applied Mathematics, vol. 172, pp. 79-99, 2004.

[112] M. Caliari, P. Kandolf, A. Ostermann, and S. Rainer, "Comparison of software for computing the action of the matrix exponential," $\mathrm{BIT} \mathrm{Nu}$ merical Mathematics, vol. 54, March 2014.

[113] A. H. Al-Mohy and N. J. Higham, "Computing the action of the matrix exponential, with an application to exponential integrators," SIAM Journal of Scientific Computing, vol. 33, pp. 488-511, March 2011.

[114] R. C. K. Friedrichs and H. Lewy, "On the partial difference equations of mathematical physics," IBM Journal of Research and Development, vol. 11, pp. 215-234, March 1967.

[115] M. Belkin, J. Sun, and Y. Wang, "Discrete laplace operator on meshed surfaces," in SCG Symposium on Computational Geometry, pp. 278-287, 2008.

[116] M. Belkin and P. Niyogi, "Towards a theoretical foundation for laplacianbased manifold methods," Journal of Computer And System Sciences, vol. 74, pp. 1289-1308, 2008. 
[117] H. Zhang, "Discrete combinatorial laplacian operators for digital geometry processing," in Proc. SIAM Conference on Geometric Design and Computing, pp. 575-592, 2004.

[118] M. Meyer, M. Desbrun, P. Schröder, and A. H. Barr, "Discrete differential geometry operators for triangulated 2-manifolds," in VisMath Proceedings, 2002.

[119] M. Wardetzky, "Convergence of the cotangent formula: An overview," Discrete Differential Geometry, vol. 28, pp. 275-286, 2008.

[120] K. Fujiwara, "Eigenvalues of laplacians on a closed riemannian manifold and its nets," Proceddings of the American Mathematical Society, vol. 123, pp. 2585-2594, August 1995.

[121] U. Pinkall and K. Poltheir, "Computing discrete minimal surfaces and their conjugates," Experimental Mathematic, vol. 2, pp. 15-36, 1993.

[122] M. Belkin, J. Sun, and Y. Wang, "Constructing laplace operator from point clouds in rd," in Proceedings of the Twentieth Annual ACM-SIAM Symposium on Discrete Algorithms, (Philadelphia, PA, USA), pp. 10311040, Society for Industrial and Applied Mathematics, 2009.

[123] T. K. Dey, P. Ranjan, and Y. Wang, "Convergence, stability, and discrete approximation of laplace spectra," in ACM-SIAM Symposium on Discrete algorithms, pp. 650-663, January 2010. 
[124] M. M. Bronstein and I. Kokkinos, "Scale-invariant heat kernel signatures for non-rigid shape recognition," in IEEE Conference on Computer Vision and Pattern Recognition, June 2010.

[125] "Standford scanning repository." http://graphics.stanford.edu/data/ 3Dscanrep/.

[126] R. Gaskell, J. Saito, M. Ishiguro, T. Kubota, T. H. N. H. S. Abe, O. Barnouin-Jha, and D. Scheeres, "Itokawa shape model v1.0. haya-amica-5-itokawashape-v1.0." https://sbn.psi.edu/pds/resource/ itokawashape.html, 2008. NASA Planetary Data System, 2008.

[127] A. Vedaldi and B. Fulkerson, "VLFeat: An open and portable library of computer vision algorithms." http://www.vlfeat.org/, 2008.

[128] A. Jagat and J. Christian, "Scale selection for vision-based relative navigation using scale space theory," in AIAA/AAS Astrodynamics Specialist Conference, September 2016.

[129] G. Zou, J. Hua, Z. Lai, X. Gu, and M. Dong, "Intrinsic geometric scale space by shape diffusion," IEEE Transactions on Visualization and Computer Graphics, vol. 15, pp. 1193-2000, November 2009.

[130] K. Mikolajczyk, Detection of local features invariant to affine transformations. PhD thesis, Institut National Polytechnique de Grenoble, 2002. 
[131] J. S. B. Mitchell, D. M. Mount, and C. H. Papadimitriou, "The discrete geodesic problem," SIAM Journal of Computing, vol. 16, pp. 647-668, August 1987.

[132] F. Tombari, S. Salti, and L. D. Stefano, "Performance evaluation of 3d keypoint detectors," International Journal of Computer Vision, vol. 102, pp. 198-220, 2013.

[133] Geodesic Exponential Kernels: When Curvature and Linearity Conflict, June 2015.

[134] R. Kalman, "A New Approach to Linear Filtering and Prediction Problems," Journal of Basic Engineering, vol. 82, pp. 35-54, 1960.

[135] R. Kalman and R. Bucy, "New Results in Linear Filtering and Prediction," Journal of Basic Engineering, vol. 83, pp. 95-108, 1961.

[136] A. Gelb, Applied Optimal Estimation. Cambridge, MA: MIT Press, 1974.

[137] E. Lefferts, F. Markley, and M. Shuster, "Kalman Filtering for Spacecraft Attitude Estimation," Journal of Guidance, Control, and Dynamics, vol. 5, no. 5, pp. 417-429, 1982.

[138] F.L. Markley and J.L. Crassidis, Fundamentals of Spacecraft Attitude Determination and Control. New York, NY: Springer, 2014. 
[139] A. Tonioni, S. Salti, F. Tombari, R. Spezialetti, and L. D. Stefano, "Learning to detect a good 3d keypoints," International Journal of Computer Vision, vol. 126, pp. 1-20, 2018.

[140] M. Magnusson, The Three-Dimensional Normal-Distributions Transform - an Efficient Representation for Registration,Surface Analysis, and Loop Detection. Ph.d., Örebro University, 2009.

[141] M. Greenacre, Correspondence Analysis in Practice. Chapman and Hall, second ed., 2007.

[142] T. Ahonen, A. Hadid, and M. Pietikäinen, "Face descriptors with local binary patters: Application to face recognition," IEEE Transactions on Pattern Analysis and Machine Intelligence, vol. 28, pp. 2037-2041, December 2006.

[143] A. Richtsfel, T. Mörwald, J. Prankl, M. Zillich, and M. Vincze, "Segmentation of unknown objects in indoor environments," in International Conference on Intelligent Robots and Systems, October 2012.

[144] S. C. Stein, M. Schoeler, J. Papon, and F. Wörgötter, "Object partitioning using local convexity," in IEEE Conference on Computer Vision and Pattern Recognition (CVPR), June 2014.

[145] S. Gupta, P. Arbeláez, R. Girshick, and J. Malik, "Indoor scene understanding with rgb-d images: Bottom-up segmentation, object detection 
and semantic segmentation," International Journal of Computer Vision, vol. 122, pp. 133-149, 2015.

[146] Y. Ioannou, B. Taati, and R. Harrap, "Difference of normals as a multiscale operator in unorganized point clouds," in 3D Imaging, Modeling, Processing, Visualization $\&$ Transmission Conference, pp. 501-508, October 2012 .

[147] R. Unnikrishnon and M. Hebert, "Multi-scale interest regions from unorganized point clouds," in IEEE Conf. on Computer Vision and Pattern Recognition (CVPR), pp. 1-8, 2008.

[148] M. Pauly, R. Keiser, and M. Gross, "Multi-scale Feature Extraction on Point-Sampled Surfaces," Computer Graphics Forum, vol. 22, no. 3, 2003.

[149] W. Wohlkinger and M. Vincze, "Ensembler of shape functions for 3d object classification," in IEEE International Conference on Robotics and Biometrics, December 2011.

[150] H. Q. Dinh and S. Kropac, "Multi-resolution spin-images," in IEEE Conference on Computer Vision and Pattern Recognition, June 2006.

[151] U. Castellani, M. Cristani, S. Fantoni, and V. Murino, "Sparse points matching by combining 3d mesh saliency with statistical descriptors," European Computer Graphics Forum, vol. 27, no. 2, pp. 643-652, 2008. 
[152] Y. Guo, F. Sohel, M. Bennamoun, M. Lu, and J. Wan, "Rotational projection statistics for 3d local surface description and object recognition," International Journal of Computer Vision, vol. 105, pp. 63-86, 2013.

[153] B. Steder, R. B. Rusu, K. Konolige, and W. Burgard, "Point feature extraction on 3d range scans taking into account object boundaries," in Proceedings of the IEEE ICRA, May 2011.

[154] A. Makadia, A. P. IV, and K. Daniilidis, "Fully automatic registration of 3d point clouds," in IEEE Computer Society Conference on Computer Vision and Pattern Recognition, pp. 1297-1304, 2006.

[155] T. Fiolka, J. Stückler, D. A. Klein, D. Schulz, and S. Behnke, "Sure: Surface entropy for distinctive 3d features," in Proceddings of Spatial Cognition, September 2012.

[156] A. Aldoma, F. Tombari, J. Prankl, A. Richtsfeld, L. D. Stefano, and M. Vincze, "Multimodal cue integration through hypotheses verification for rgb-d object recognition and 6dof pose estimation," in International Conference on Robotics and Automation (ICRA), May 2013.

[157] L. A. Alexandre, "3d descriptors for object and category recognition: A comparative evaluation," in IEEE/RSJ International Conference on Intelligent Robots and Systems (IROS, 2012.

[158] A. Ceron and F. Prieto, "Evaluating and comparing of 3d shape descriptors for object recognition," in Advances in Visual Computing, pp. 484- 
492, Springer Berlin Heidelberg, 2013.

[159] R. Hänsch, T. Weber, and O. Hellwich, "Comparison of 3d interest point detectors and descriptors for point cloud fusion," in ISPRS Annals of Photogrammetry, Remote Sensing and Spatial Information Sciences, vol. II-3, pp. 57-64, 092014.

[160] X.-F. Han, J. S. Jin, J. Xie, M.-J. Wang, and W. Jiang, "A comprehensive review of 3d point cloud descriptors," ArXiv, 2018. 


\section{Vita}

Andrew Patrick Rhodes was born the $24^{\text {th }}$ of February, 1990 in Morgantown, West Virgina to Walter C. Rhodes and Diane M. Rhodes. He graduated Magna Cum Laude from West Virginia University in December 2013, receiving a Bachelor of Science in Mechanical Engineering, a Bachelor of Science in Aerospace Engineering, and a minor in Spanish Language. While at WVU, he worked under the guidance of Dr. John Christian in the Applied Space Exploration Laboratory (ASEL). He has completed two summer internships at the NASA Johnson Spaceflight Center in Houston, TX, one internship at the AFRL on Kirtland Air Force Base in Albuquerque, NM, and one internship at the ATRC of Wright State University in Dayton, OH. Andrew is an alumnus of the Pride of West Virginia Mountaineer Marching Band, marching the sousaphone from 2011 to 2013. He also played the spiderweb lead pan in the WVU Steel Drum Ensemble for a total of eighteen semesters. In 2019, he won the West Virginia Business Plan Competition for the Hospitality and Tourism category.

Permanent address: 604 Elmina Street

Morgantown, West Virginia 26505

This dissertation was typeset with $\mathrm{AT}_{\mathrm{E}} \mathrm{X}^{\dagger}$ by the author.

${ }^{\dagger} \mathrm{LAT}_{\mathrm{E} X}$ is a document preparation system developed by Leslie Lamport as a special version of Donald Knuth's TEX Program. 